\title{
Wahrnehmung und Vorstellung von Bewegungen - Studien im Kontext des Erwerbs sportlicher Fertigkeiten in der Kindheit
}

\author{
Dissertation \\ zur Erlangung des sozialwissenschaftlichen Doktorgrades \\ der Sozialwissenschaftlichen Fakultät \\ der Georg-August-Universität Göttingen
}

vorgelegt von Sandra Salb

aus Leinefelde

Göttingen, 2015 


\section{Inhaltsverzeichnis}

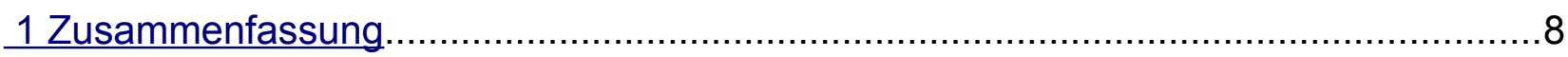

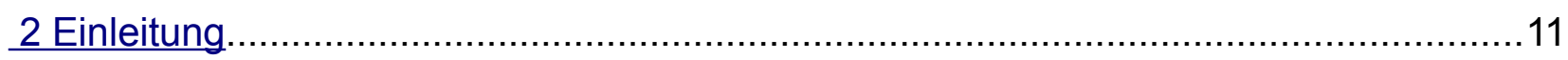

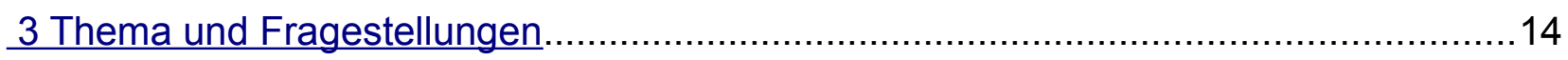

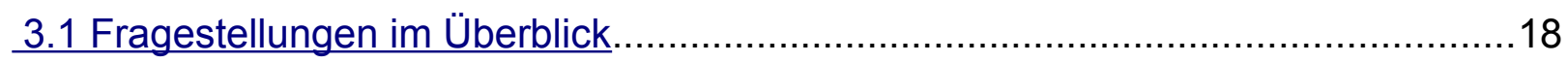

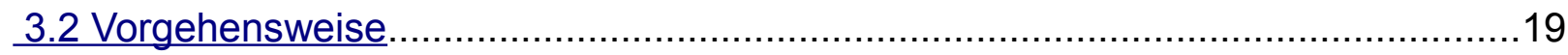

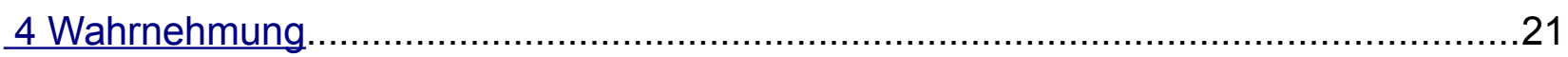

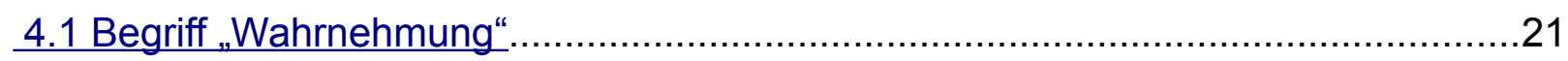

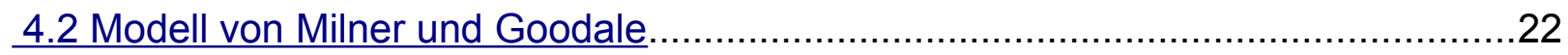

4.3 Organisationsprinzipien der Wahrnehmung ..................................................23

4.4 Geschlechterunterschiede in der Wahrnehmung_.........................................24

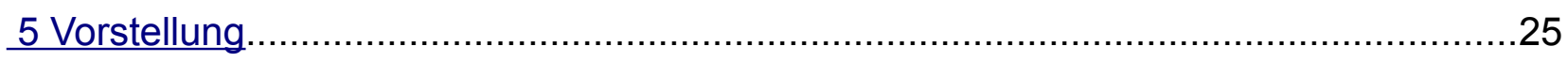

5.1 Begriff .Vorstellung

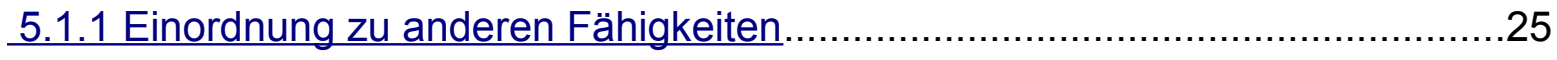

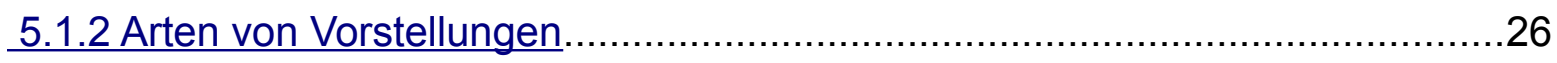

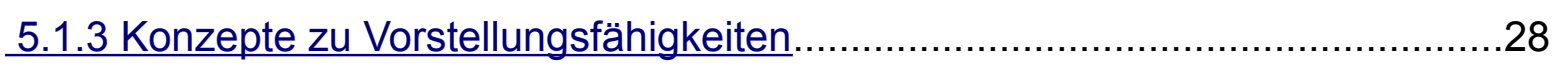

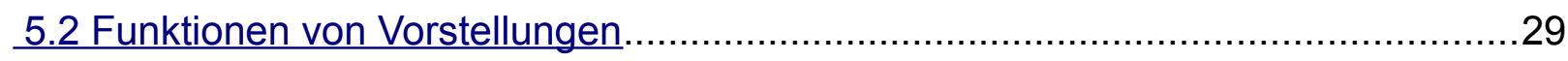

5.3 Untersuchungsverfahren zur Messung von Vorstellungen.....................................30

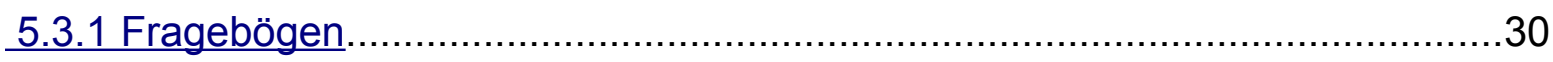

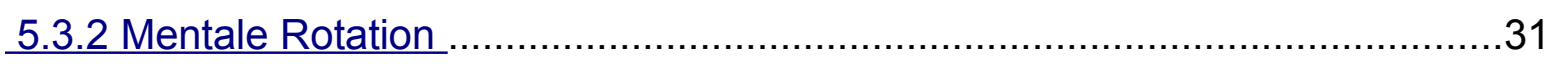

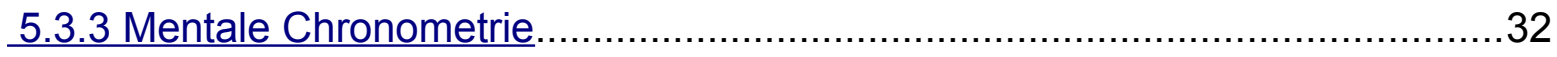

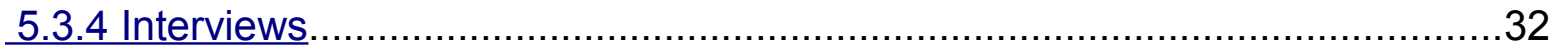

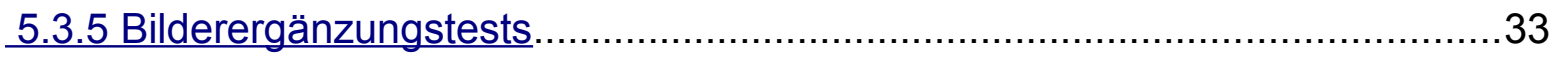

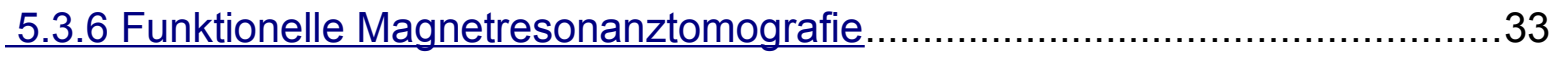

5.3.7 Entwicklung einer alternativen Messmethode ……....................................34

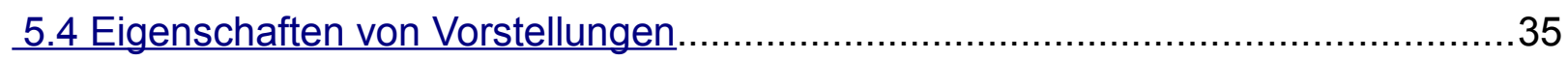

5.4.1 Repräsentationsformat von Vorstellungen: bildlich versus symbolisch..........35

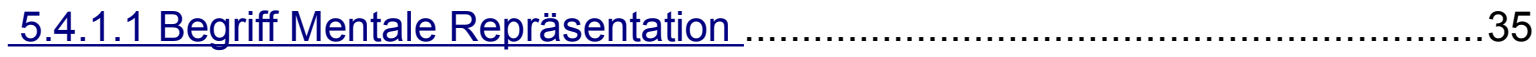

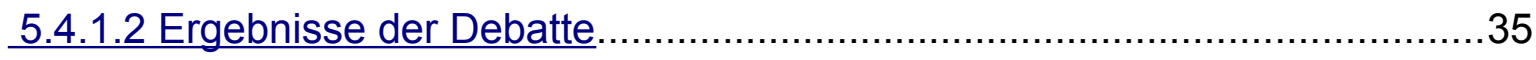

5.4.2 Sind Vorstellungen bewusst? ................................................................ 


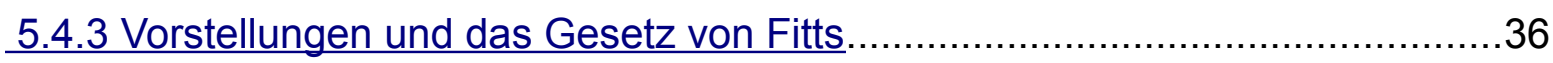

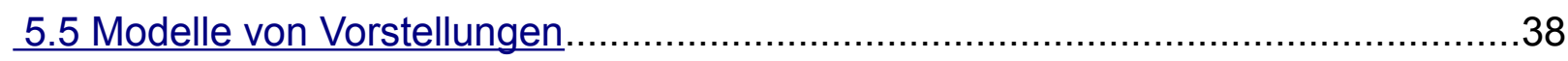

6 Vergleich von Wahrnehmung und Vorstellung ......................................................42

6.1 Gemeinsamkeiten von Wahrnehmung und Vorstellung....................................42

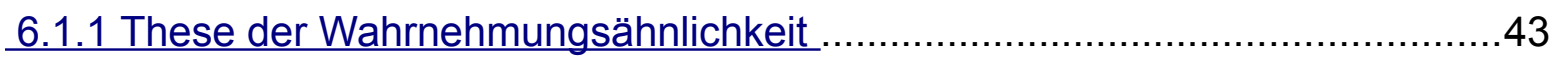

6.1.2 Zeitliche Ähnlichkeit und biomechanische Gesetze.....................................44

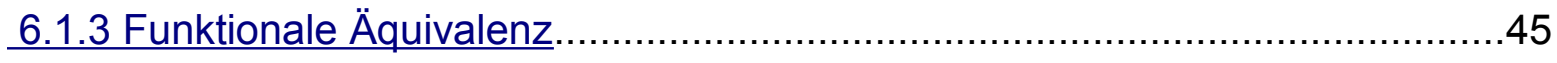

6.1.4 Topografische Organisation von Wahrnehmung und Vorstellung..................47

6.2 Unterschiede von Wahrnehmung und Vorstellung ...........................................49

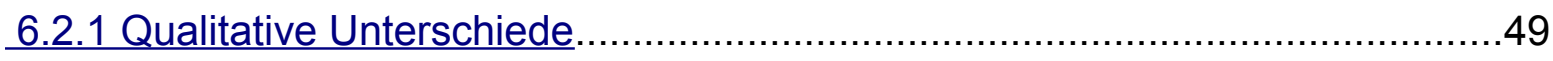

6.2.2 Quantitative Unterschiede................................................................

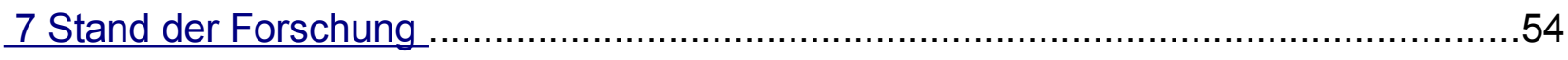

7.1 Motorik, Wahrnehmung und Vorstellung ………..................................................54

7.1.1 Motorische Fertigkeiten als abhängige Variable .........................................54

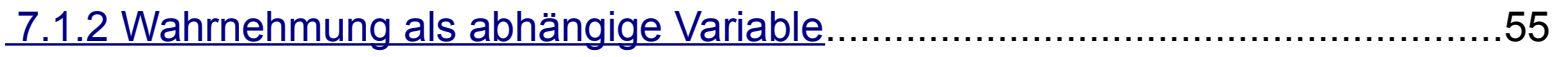

7.1.3 Vorstellungen als abhängige Variable ........................................................56

7.2 Vorstellung und Gedächtnis: Interferenzeffekte .................................................58

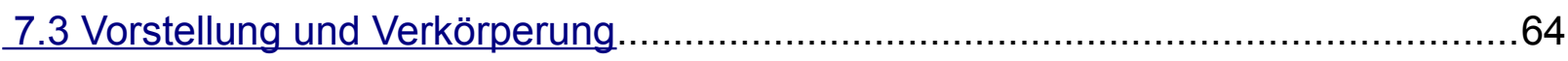

7.4 Zusammenhang von Vorstellung und motorischen Fertigkeiten in der Kindheit....68

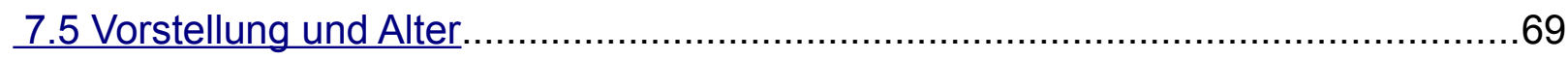

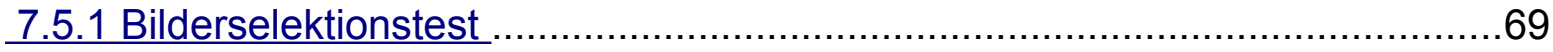

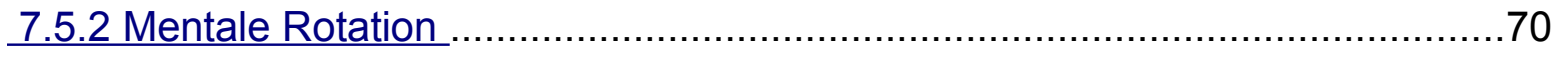

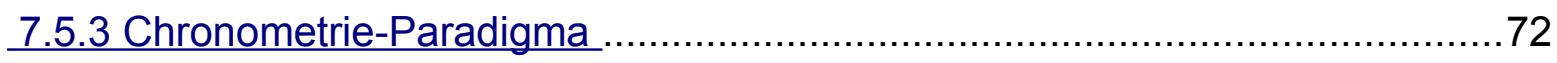

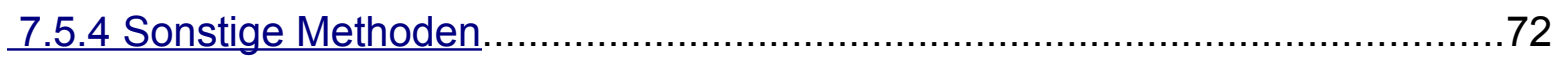

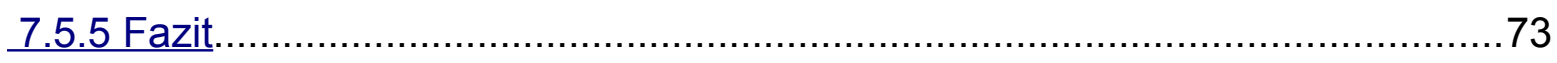

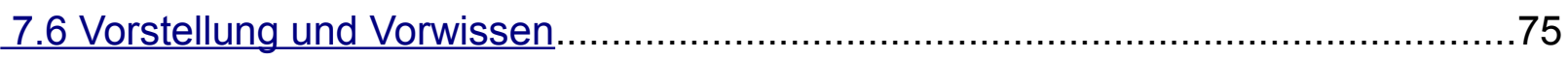

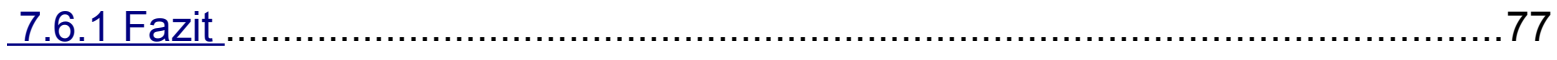

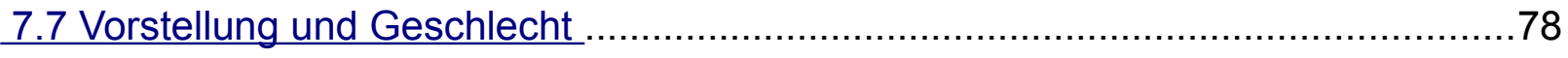

7.7.1 Mögliche Ursachen für Geschlechterunterschiede .....................................78

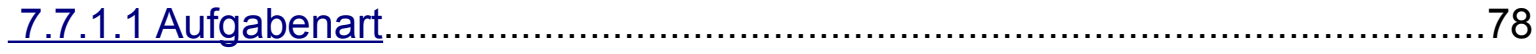

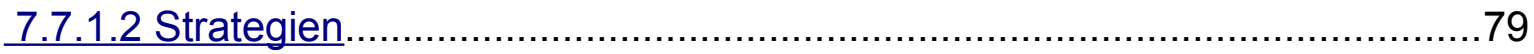


7.7.1.4 Lateralisierung und andere Entwicklungsfaktoren.................................82

7.7.1.5 Einfluss der Instruktion und stereotype Erwartungen..............................82

7.7.2 Mentalen Rotation und Geschlechterunterschiede bei Kindern ....................83

7.7.3 Befunde sonstiger Methoden bei Kindern ……................................... 84

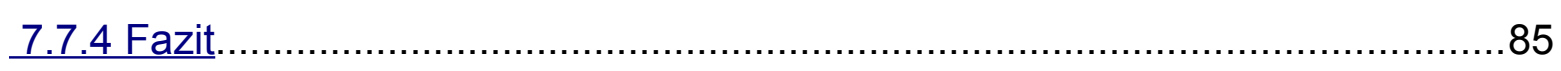

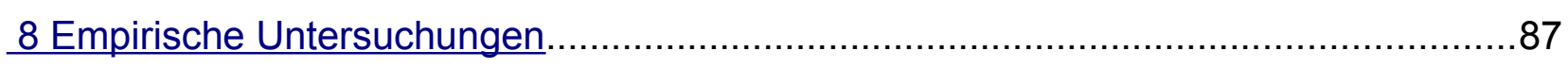

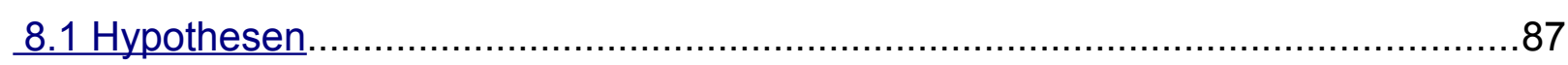

8.2 Ablauf und Versuchsplanung der Gesamtuntersuchung..................................90

8.3 Studie 1: Zum Zusammenhang motorischer und kognitiver Leistungen in der

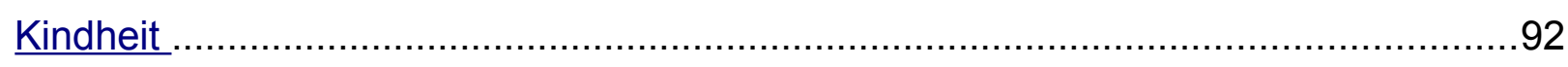

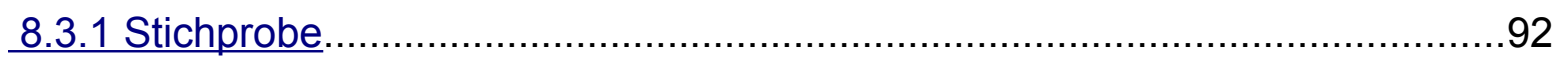

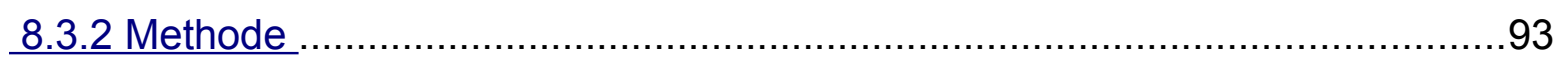

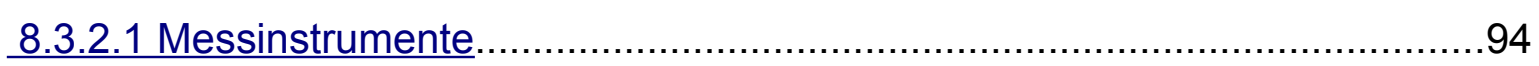

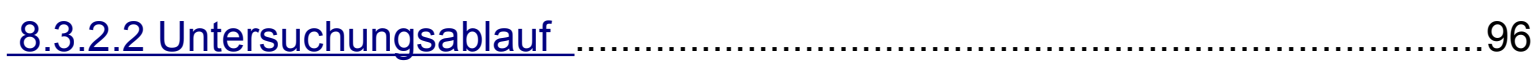

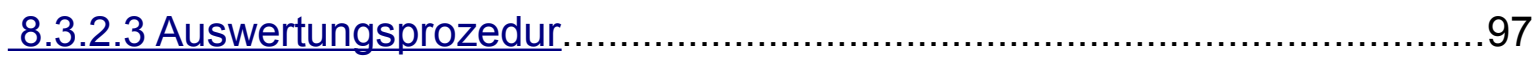

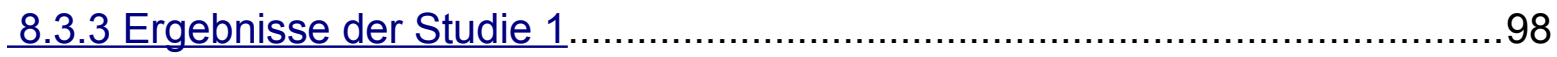

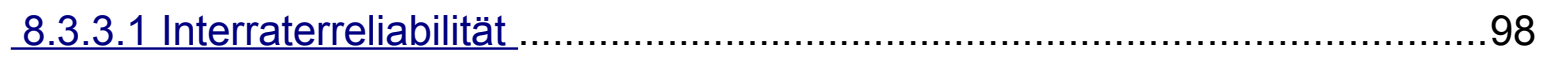

8.3.3.2 Bewegungslernen über 3 Messzeitpunkte (Gesamtskala).......................99

8.3.3.3 Bewegungslernen über 3 Messzeitpunkte (Subskalen)........................100

8.3.3.4 Korrelationen zum MZP2 und MZP3 (Gesamtskala).............................104

8.3.3.5 Korrelationen zum MZP2 (Subskalen) ……......................................105

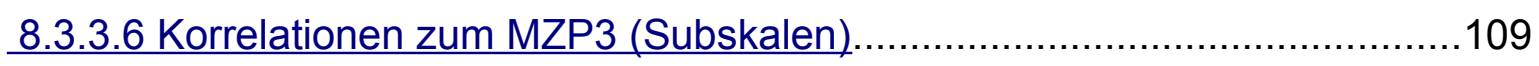

8.3.3.7 Einzelfallanalyse zum Bewegungslernen ..............................................113

8.3.3.8 Merkmale der Tennistechnik als Lernindikatoren ..................................114

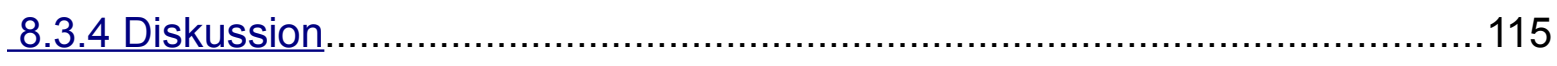

8.4 Studie 2: Einfluss von Erfahrung auf Wahrnehmung und Vorstellung von Bewegungen in der Kindheit .........................................................................117

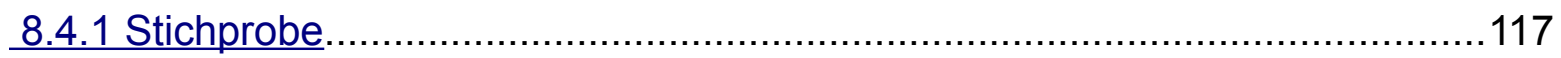

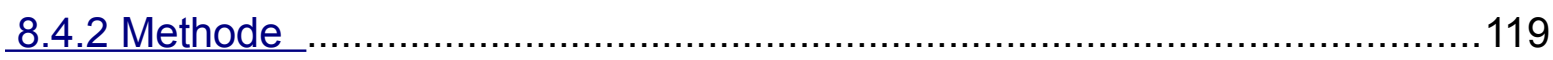

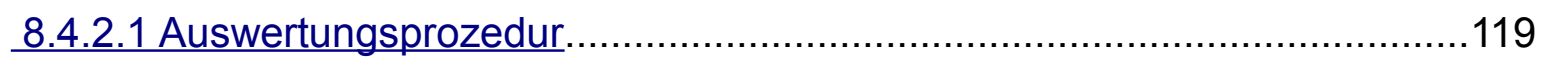

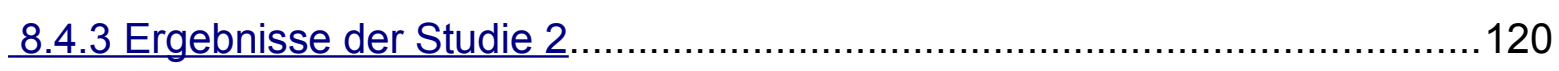

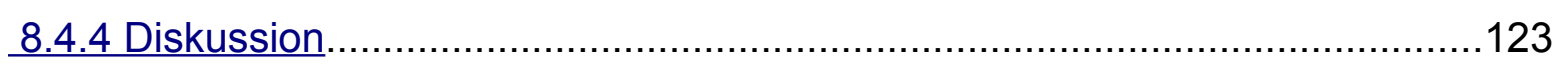


8.5 Studie 3: Einfluss des Geschlechts auf Wahrnehmung und Vorstellung von

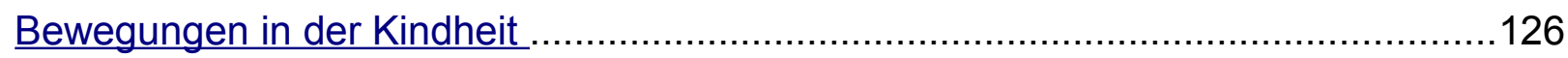

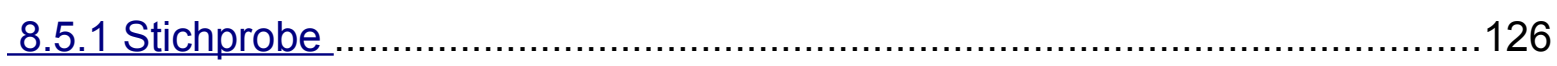

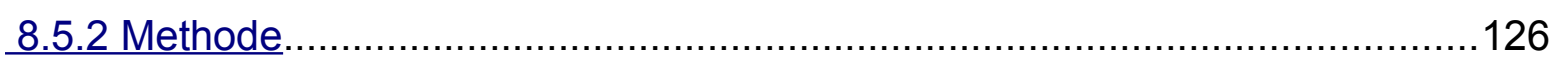

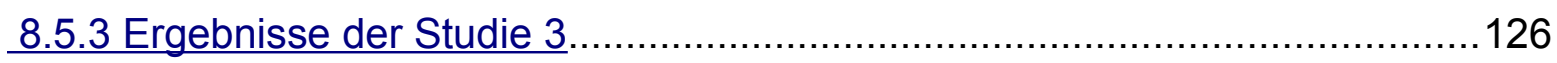

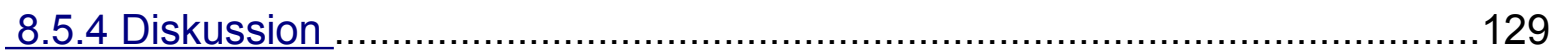

8.6 Studie 4: Einfluss der Versuchsbedingung auf Wahrnehmung und Vorstellung von Bewegungen in der Kindheit ...................................................................... 132

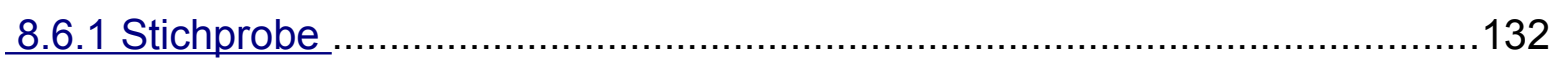

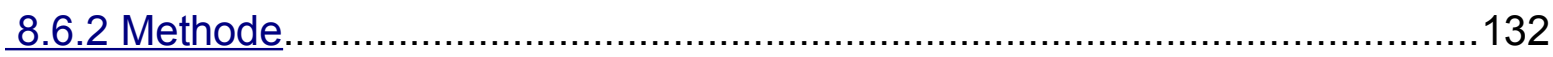

8.6.3 Ergebnisse der Studie 4................................................................. 132

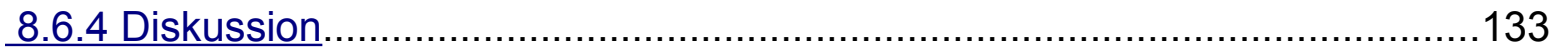

8.7 Studie 5: Einfluss von Trainingserfahrung auf Wahrnehmungen und Vorstellung von Bewegungen in der Versuchsgruppe.................................................... 135

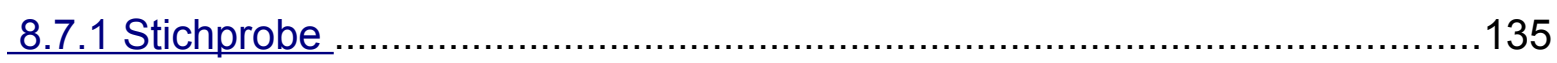

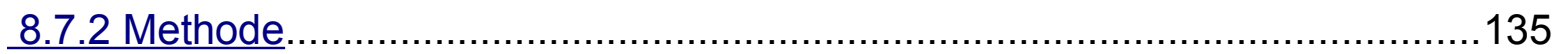

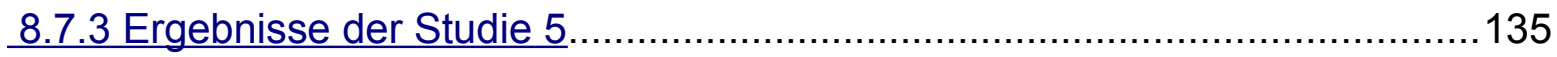

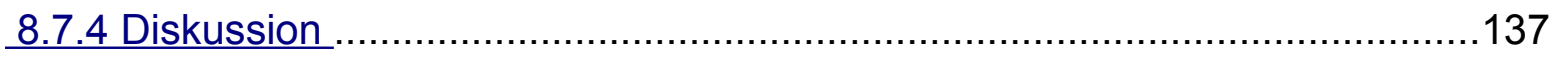

8.8 Studie 6: Einfluss von Trainingserfahrung auf Bewegungslernen in der

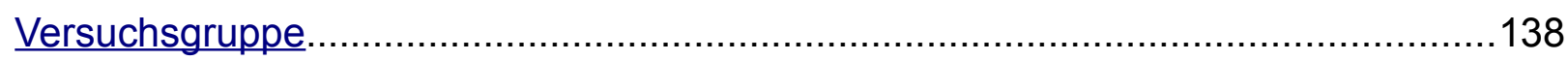

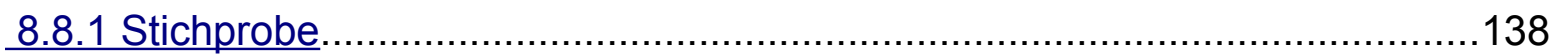

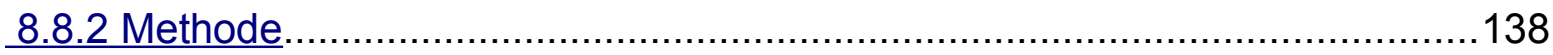

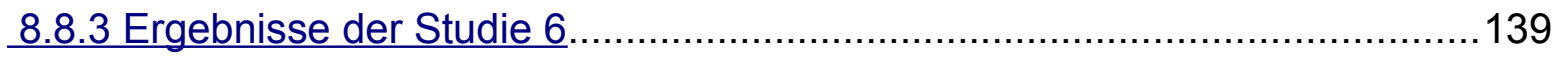

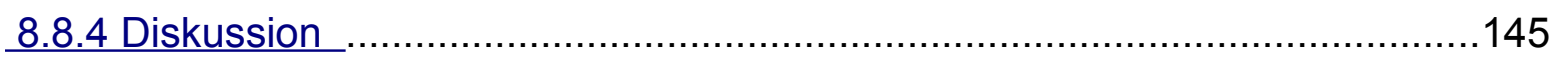

8.9 Studie 7: Einfluss des Geschlechts auf das Bewegungslernen in der

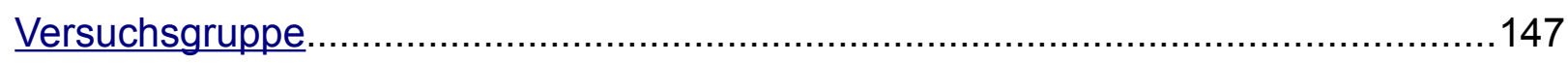

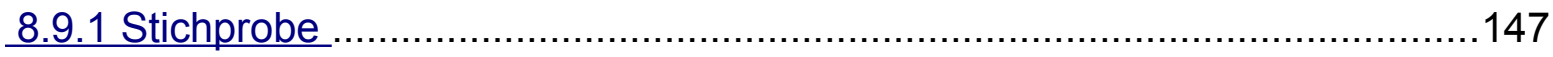

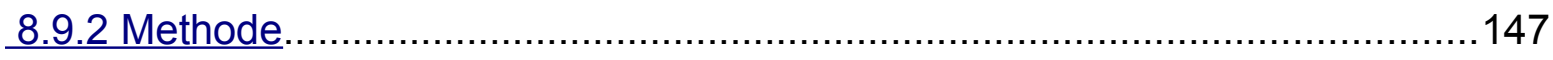

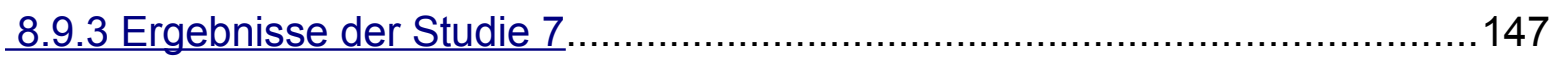

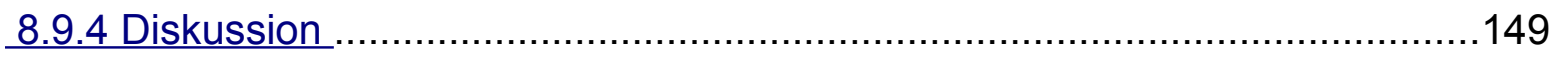

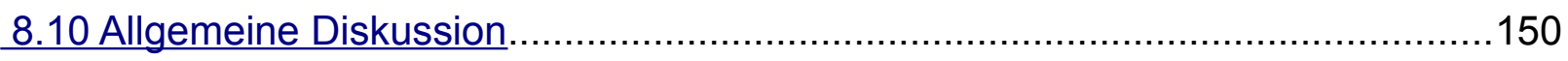

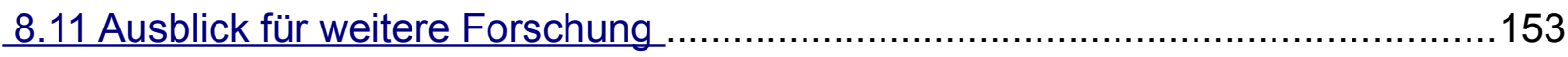


9.1 Bewegungstagebuch und Fragebogen zur Erfassung der Tennistechnik............154

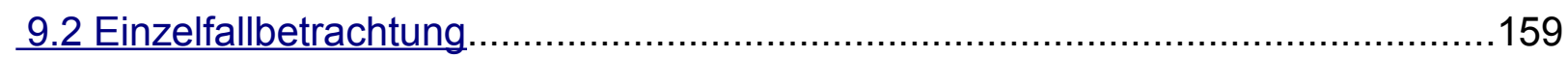

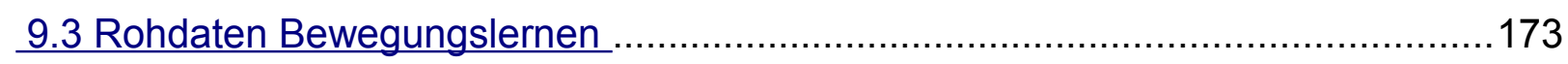

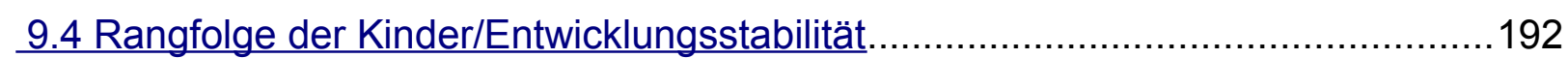

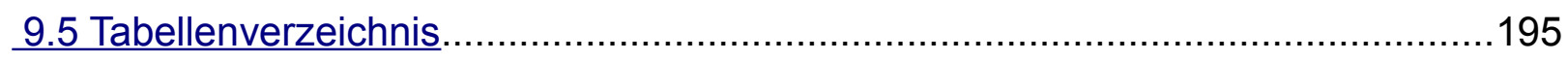

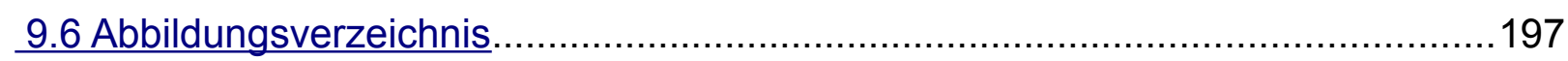

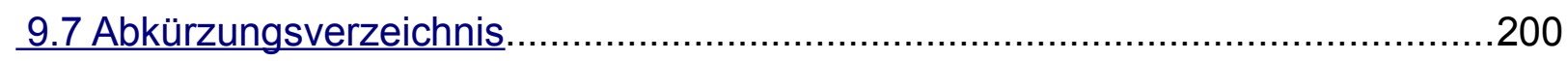

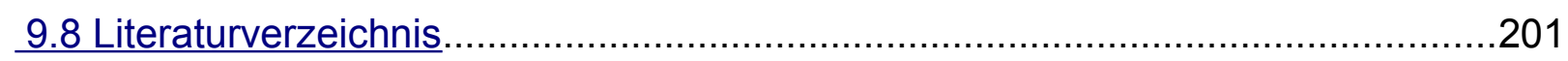

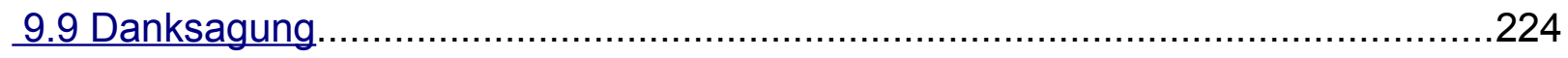


1. Gutachter: Prof. Dr. Herbert Hopf

2. Gutachter: Prof. Dr. Andreas Bund

3. Gutachter: Prof. Dr. Gerd Thienes

Tag der mündlichen Prüfung: 7. Mai 2014 
„Mental images - like all mental events- are notoriously difficult to put on public display, [...] But electrons, quarks, and black holes are also difficult to put on public display [...]" (Kosslyn, 1994, S. 2). 


\section{Zusammenfassung}

Die Entwicklung einer neuen Methode zur Messung von Vorstellungen steht im Zentrum dieser Arbeit. Es wurden damit zum einen motorische und kognitive Leistungszusammenhänge bei Kindern, die das Tennisspiel erlernen, untersucht. Dabei ging es um Bewegungsfertigkeiten zu verschiedenen Messzeitpunkten und deren Zusammenhang mit der Wahrnehmungs- und Vorstellungsleistung (Studie 1). Die Vorstellungsleistung wurde dabei mit der neuen Methode untersucht. Sie wurde an jeweils 3 Tagen in aufeinanderfolgenden Wochen mittels auf einem Bildschirm präsentierter Videopaare untersucht, welche eine Bewegungssequenz (einen TennisVorhandschlag) zeigten. Dabei waren Teile der Bewegung verdeckt. Aufgabe der Kinder war es zu beurteilen, ob die Videopaare unabhängig von der Abdeckung gleich sind. Die Ratewahrscheinlichkeit betrug 50\%. In der Wahrnehmungsbedingung (Basisbedingung) wurden die Videopaare ohne Abdeckung gezeigt. Im Ergebnis konnten Zusammenhänge zwischen Wahrnehmungs- und Vorstellungsleistungen und dem Bewegungslernen bei einzelnen Teilfertigkeiten des Bewegungslernens sowie bestimmten experimentellen Bedingungen aufgezeigt werden.

Zum anderen wurde der Einfluss von Erfahrung auf das Beobachten und Sich-Vorstellen von Bewegungen im Rahmen der hier durchgeführten quasi-experimentellen Studie untersucht (Studie 2). Dabei wurden Lernanfänger im Tennis mit motorischer und visueller Erfahrung und Kinder, die keine solchen Erfahrungen mit dem Tennisspiel hatten, mittels eines Novizen-Experten-Paradigmas unter Berücksichtigung des Faktors Geschlecht verglichen (Studie 3). Im Ergebnis hatten die Kinder ohne Erfahrungen mit dem Tennisspiel einen Vorteil bei der Lösung der Wahrnehmungs- und Vorstellungsaufgabe. Mädchen schnitten durchschnittlich besser ab als Jungen. Insbesondere schnitten die Mädchen der Kontrollgruppe besser $a b$ als die Mädchen der Versuchsgruppe. Dies wird hinsichtlich unterschiedlicher geschlechtsspezifischer Strategien bei der Aufgabenlösung interpretiert. Es scheint aber auch schwieriger für die Tennis spielenden Kinder zu sein, ihre motorischen Erfahrungen zu aktivieren und diese in Form von neuen Vorstellungen zu generieren.

In der Studie 4 wurde der Einfluss der Versuchsbedingung auf die Wahrnehmung und Vorstellung von Bewegungen in der Kindheit untersucht. Es konnte der Unterschied zwischen Wahrnehmungs- und Vorstellungsleistungen quantifiziert werden, dabei spielt das Geschlecht eine Rolle als Moderatorvariable: Jungen in diesem Alter weisen 
demnach quantifizierbare Unterschiede zwischen der Wahrnehmung und der Vorstellungen auf, während diese bei Mädchen diesen Alters ähnlich zu sein scheinen.

In der Studie 5 wurde geprüft, ob die in zwei Trainingsgruppen aufgeteilten Tenniskinder hinsichtlich der Wahrnehmung und Vorstellung vergleichbar sind, was der Fall war.

In den weiteren Studien wurde der Einfluss von Erfahrung (Studie 6) und Geschlecht (Studie 7) auf das Bewegungslernen erfasst. Beide Variablen hatten in diesem Alter keinen Einfluss auf das Erlernen von Bewegungen.

Die Ergebnisse liefern erste Hinweise für den Vollzug einer dynamischen mentalen Vorstellung, die mit dieser Methode gemessen werden kann.

\section{Summary}

The core of the thesis was the development of a new method to measure mental images of movements. First children's motor and cognitive achievements were explored while they were learning tennis. Within this approach motor skills at different times were recorded and correlated with perception and imagery skills (study 1). The latter was investigated by a new method. The imagery skills were recorded during three consecutive weeks by pairs of videos presented on a computer screen, which showed a movement sequence (a tennis forehand stroke). Parts of the movement were covered by a mask. The Children's task was to judge whether pairs of videos were the same regardless of the cover. The chance of guessing was $50 \%$. In the perception condition (basic condition) video pairs were shown without a cover. As a result correlations between some imagery and perceptual conditions and some motor skills could be found.

Second the influence of experience on perception and imaging of movements was explored by quasi-experimental study (study 2). To research this, children who had beginner's motor and visual experience with tennis were compared to children without that experience by novice-expert-paradigm and with respect to the factor gender (study 3). As a result children without experience in tennis had an advantage in the solution of the perception and imagery tasks; girls were on average better than boys. This was explained by their different strategy use. At the same time it seems to be difficult for the beginners to recall motor experiences and create new ones. Results give evidence of complementary skills in mental imagery and perception.

In the study 4 the influence of the experimental condition on perception and mental imagery was experienced. The difference between perceptional and mental imagery 
skills could be quantified, whereas gender played a role as a moderator variable. Accordingly, boys but not girls showed differences in these functions, while perception and imagery seemed to have common characteristics in girls at this age.

In the study 5 it was identified, weather the two training groups were comparable by their perceptional and imagery abilities, which was the case.

Based on previous work, the learning of movements was measured according to the factors experience (study 6) and gender (study 7). No differences were found between the girls and the boys regarding the learning of motor skills. Experience also had no influence on motor skill learning.

The results show first evidence of measuring dynamic mental imagery by this method. 


\section{Einleitung}

Vorstellungen haben Einfluss auf das Erlernen von Bewegungen, denn Studien zeigen die Effektivität mentalen Trainings, bei dem eine mentale Simulation von Umgebung und/oder Bewegungsmerkmalen zu einer besseren Ausführung der Bewegung führt (z.B. die Metaanalyse von Feltz und Landers, 1983). Auch Kosslyn, Behrmann und Jeannerod (1995) betonen die Rolle von mentalen Vorstellungen nicht nur in den Bereichen des Denkens und des Gedächtnisses, sondern auch für das Lernen und den Fertigkeitserwerb. Wenige Untersuchungen gibt es allerdings hierzu bei Lernanfängern in der Kindheit.

Es wurde noch in den 1970er Jahren die Auffassung vertreten, dass Vorstellungen nur durch die Schilderung und das eigene Erleben erfahrbar sein könnten (z.B. Bosshardt, 1973). In dieser Dissertation wird ein quasi-experimenteller und damit anderer Zugang zu Vorstellungen gewählt. Damit sollen Vorstellungen mit Wahrnehmungen verglichen und Unterschiede quantifiziert werden.

Vorstellungsleistungen $\mathrm{zu}$ untersuchen ist deshalb eine Herausforderung, weil Menschen, wenn sie introspektiv gefragt werden, unterschiedliche Vorstellungen bilden. Auch sind Vorstellungsbilder über die Zeit veränderbar, können sogar bewusst verändert werden und sind so gerade für Kinder schwer beschreibbar. Bei Kindern kann außerdem hinzukommen, dass Aufgaben möglicherweise nicht verstanden und nicht der Intention nach umgesetzt werden.

\section{Motorik und Kognition}

So ist für die sportwissenschaftliche Forschung besonders interessant, wie Vorstellungsfähigkeiten mit Bewegungslernen zusammenhängen. Die Art und Weise, in welcher sich die motorischen und die kognitiven Leistungen beim Lernen von Bewegungsfertigkeiten gegenseitig beeinflussen, ist ein in den letzten Jahren wieder entdecktes Forschungsfeld. Dabei werden unter jenen motorischen Fertigkeiten der Grad der Beherrschung von Bewegungsabfolgen und -merkmalen verstanden, die für die Ausübung einer Sportart notwendig sind.

Interessant und noch wenig erforscht ist der Zusammenhang von Vorstellungen und Bewegungsfertigkeiten bei Kindern. Das Alter und die Erfahrung sind möglicherweise wichtige Determinanten dieser Beziehung. Etwa fand Caeyenberghs und Kollegen (2009a) einen mit dem Alter stärker werdenden Zusammenhang zwischen Vorstellungen und motorischen Fertigkeiten. Einige Untersuchungen stellen heraus, dass Kinder 
bereits im Alter von 5 Jahren mentale Vorstellungen erzeugen können (Kosslyn et al., 1990; Marmor, 1975). Noch ist unklar, ob Kinder dieser Altersgruppen bereits Vorstellungen für das Bewegungslernen nutzen. Bohan et al. (1999) behauptet, dass das Mentale Training bei zunehmender Beherrschung der Fertigkeit weniger Effekte zeigt. Das würde bedeuten, dass junge Kinder mehr von dem mentalen Training profitieren als ältere Kinder. Bezüglich Lernanfängern widersprechen jedoch die Autoren Mulder et al. (2004). Beim Erlernen einer neuen Bewegung spielt die motorische Vorstellung zumindest bei erwachsenen Novizen keine Rolle (Novizen sind hier Menschen ohne Erfahrung mit einer bestimmten Bewegungsabfolge, für den Begriff motorische Vorstellung siehe Kap. 5.1.2). Fraglich ist jedoch, inwiefern das Lernen von Erwachsenen mit denen von Kindern verglichen werden kann, wobei dies nicht Gegenstand dieser Dissertation ist.

\section{Erfahrung und Vorstellungen}

Sportler beherrschen nicht nur bestimmte Bewegungsfertigkeiten, sondern sie verfügen durch die Beobachtung anderer oder sich selbst zum Beispiel im Training, im Spiegel oder per Videofeedback auch über visuelle Erfahrungen. Erfahrung wird in dieser Arbeit somit als kombinierte visuelle und motorische Erfahrung verstanden, die einerseits bei der Exposition mit bestimmten Bewegungsreizen und andererseits bei der Ausübung bestimmter Bewegungsaktivitäten entsteht und im Gedächtnis als Form einer Bewegungsrepräsentation gespeichert wird. Bewegungsrepräsentationen werden als im Gedächtnis gespeichertes Wissen von Bewegungen verstanden (Munzert et al., 2009). Es wird zunächst angenommen, dass Kinder, die mehr motorische und visuelle Erfahrungen mit den Tennisbewegungen haben, sich diese Bewegungen auch besser vorstellen können.

\section{Geschlechterunterschiede}

Aufgrund von Voruntersuchungen anderer Gruppen wird angenommen, dass Mädchen bei dynamischen Vorstellungen hinter den Jungen zurückliegen (siehe Kap. 7.7).

Andererseits gibt es Hinweise darauf, dass Geschlechterunterschiede bei den in der Vorstellung rotierten Gegenständen erst in der Adoleszenz und im Erwachsenenalter bedeutsam werden (vgl. ebenfalls Kap. 7.7). Daraus folgt, dass es bei jüngeren Kindern weniger große Unterschiede gibt. Eine andere Lesart wäre die, dass das Verfahren der Mentalen Rotation wenig geeignet ist, Geschlechterunterschiede bei jüngeren Kindern aufzudecken. Dies ist einer von mehreren Gründen, ein neues Verfahren zur Messung von Vorstellungen zu entwickeln. Weitere Gründe liegen in den Nachteilen anderer 
Messverfahren (siehe dazu Kap. 5.3).

Vergleich der Versuchsbedingungen

Auch der Vergleich von Wahrnehmungs- und Vorstellungsleistungen ist ein stärker in den Fokus gerücktes Forschungsfeld. Dabei konnte gezeigt werden, dass Wahrnehmungsvorgänge und Vorstellungen große Ähnlichkeiten aufweisen, sich aber auch in einigen Punkten unterscheiden (siehe Kap. 6). Eine beispielhafte Studie, die Unterschiede quantifizierbar macht, ist in Kap. 6.2.2 beschrieben. Ein Vergleich von Wahrnehmung und Vorstellung, mittels eines in dieser Dissertation neu entwickelten Verfahrens, soll versuchen, bei allen Gemeinsamkeiten auch deren Unterschiede zu quantifizieren. Dabei basiert das Prinzip der neuen Methode auf mentaler Ergänzung visueller Teilinformation (siehe Kap. 5.3.7 für das zugrundeliegende Prinzip und Kap. 8.3.2 für die Erläuterung des Messinstruments und des Untersuchungsablaufs). 


\section{Thema und Fragestellungen}

Diese Untersuchung wurde im Rahmen eines Projektes „Interne Bewegungsrepräsentationen am Beispiel Kindertennis“ (kurz: „Jüngsten-Tennis“) durchgeführt. Dabei erlernten Kinder ab dem Alter von 4 Jahren spielerisch das Tennisspiel. Hierbei wurde als Untersuchungsthema die Wahrnehmung und Vorstellung von Bewegungen gewählt.

Es boten sich einige Vorteile, wieso gerade das Tennisspiel hier untersucht werden sollte: Es werden hierbei sowohl motorische als auch kognitive Fähigkeiten und Fertigkeiten ausgebildet. Motorische werden etwa gebraucht, um zunächst den Ball mit dem Schläger zu treffen und den Schläger als verlängerten Arm zu nutzen. Kognitive werden gebraucht, um die richtige Taktik zu erreichen. So ist etwa zu entscheiden ob die Ballannahme mittels Vorhand oder Rückhand geschehen soll. Die kognitiven Fertigkeiten beinhalten also auch antizipatorische Elemente, die es dem Spieler ermöglichen, trotz der hohen Ballgeschwindigkeiten beim Tennisspiel noch rechtzeitig reagieren zu können. So ist beim Tennis das rechtzeitige Wahrnehmen von Signalen für die richtige motorische Reaktion von Vorteil. Es kann der Aufschlag des Gegners analysiert werden, also die Drehung der Schulter oder die Stellung der Schlägerfläche bei verschiedenen Schlagtechniken (z.B. gerader Aufschlag, Twist, Slice), um die Flugbahn des Balls voraus zu nehmen.

Im Zentrum der Betrachtung standen demnach sowohl motorische als auch kognitive Leistungen der Kinder. Der Begriff der motorischen Kognition (motor cognition) beschreibt dabei das Aufgabenfeld:

„Motor cognition encompasses all the mental processes involved in the planning, preparation, and production of our own actions, as well as the mental processes involved in anticipating, predicting, and interpretation the actions of others" (Smith \& Kosslyn, 2009, S. 453).

Allerdings sollte sich dieser Begriff nicht nur auf Handlungen Anderer beziehen, sondern auf eigene Handlungen erweitert werden, wie nachfolgend aufgezeigt wird.

Calvo-Merino et al. $(2004,2006)$ haben in ihrer früheren Studie gezeigt, dass Balletttänzer bzw. Capoeira-Tänzer, welche sich selbst beim Training sahen, besser abschnitten, als wenn sie eine ihnen unbekannte (die jeweils andere) Sportart zu Gesicht bekamen. In der späteren Studie konnte der Einfluss von visueller und motorischer 
Erfahrung getrennt betrachtet werden: Indem weibliche Ballettänzer ihre eigenen Bewegungen sahen und dabei besser abschnitten als die innen visuell ebenso vertrauten, aber in der motorischen Ausführung unbekannten rein männlichen Bewegungen.

Auch aus der Musik gibt es hierzu Beispiele: Keller, Knoblich und Repp (2007) untersuchten Pianisten. Hierbei wurden audiovisuelle Vorstellungen untersucht. Die Musiker sollten ein Duett spielen, wobei der komplementäre Duettpartner vorher aufgenommen wurde. In einem Teil der Fälle wurde das Duett vom Pianisten selbst, in den anderen Fällen von einem anderen Pianisten gespielt. Dabei konnte der Pianist seinen selbst gespielten Teil besser synchronisieren und besser erkennen als die der anderen Pianisten. Außerdem waren beide Variablen korreliert: eine bessere Synchronisierungsleistung mit der besseren Wiedererkennung des eigenen Teils des Duetts.

In dieser Dissertation wurde nach einem Ansatz und einer geeigneten Methode gesucht, welche im Rahmen des Projekts genutzt werden sollten.

Im Kontext des Bewegungslernens wird die Bedeutung von Vorstellungen für den Fertigkeitserwerb hervorgehoben. Dabei wird in dieser Dissertation angenommen, dass die Grundlage dieses Lernens die Spiegelneuronen sind. Diese Annahme kann jedoch hier nicht falsifiziert werden. Dennoch ergibt die Beschäftigung mit den mirror neurons einen weiteren Ansatzpunkt für diese Untersuchung, wie im Folgenden zu zeigen ist.

Spiegelneurone sind die Neurone, die aktiv sind, wenn etwas beobachtet wird und diese Handlung von dem Individuum ausgeführt wird. Laut Pineda sind Spiegelneuronen ein System, das per Beobachtung Bewegungswissen aktiviert: [...] a system that evokes motor representations by movement observation" (Pineda, 2008, S. 2). Bei Pineda (2008) heißt es weiter: "That is, if motor actions already exist as part of the observer agent's movement repertoire then observation of action, even when partially triggered, can be sufficient to evoke the representation“ (Pineda, 2008, S. 2).

Das bedeutet, dass für das Erlernen von Bewegungen sowohl die eigene Bewegungserfahrung als auch das Beobachten vorausgesetzt werden.

Die Entdeckung der Spiegelneurone, denen die Funktionen des „Sich-in-jemandenHineinversetzens“ zugeschrieben wird, also für die soziale Interaktion und Empathie von Bedeutung sein könnte, kann somit auf das Bewegungslernen und den Erwerb sportlicher Fertigkeiten übertragen werden, wenn es um antizipatorische Eigenschaften geht. 


\section{Notwendigkeit zur Entwicklung einer Methode}

Methoden zur Messung von Vorstellungen gibt es zwar vielfältige, jedoch sind sie meist aufwendig und teuer (insbesondere die neurophysiologischen Verfahren). Etwa wird das Standardverfahren, die funktionelle Magnetresonanztomografie (fMRT), hinsichtlich verschiedener Mängel bei der Objektivität, Reliabilität und Validität kritisiert (siehe Kap. 5.3.6). Andere Methoden sind zwar weniger kostenintensiv, kommen aber nicht ohne Rückgriff auf bestimmte sprachliche Kompetenzen aus (z.B. Interviewtechniken, Protokolle lauten Denkens). Problematisch ist auch die Verwendung von introspektiven Visualisierungstechniken innerhalb bestimmter Fragebögen (z.B. dem „Vividness of Mental Imagery Questionnaire“ von Marks, 1973) oder dem mentalen Training, denn wenig kontrollierbar ist hier, was sich die Probanden wirklich vorstellen. Das macht die Untersuchung von Kindern ab dem Alter von 4 Jahren während ihres Tennistrainings schwierig.

Für die Entwicklung der Methode zur Messung von Vorstellungen beim Erwerb sportlicher Fertigkeiten wurden deshalb folgende Kriterien zugrunde gelegt: a) der Vorstellungsinhalt soll kontrollierbar sein, b) der finanzielle Rahmen soll überschaubar bleiben und c) eine einfache Anwendbarkeit soll gegeben sein, um Kinder ab 4 Jahren während ihres Trainings untersuchen zu können.

Vorstellungen von Bewegungen werden als Simulation von Handlungen verstanden (Jeannerod, 2001). So ist naheliegend, diese Vorstellungen als mentale Ergänzungsleistungen zu operationalisieren. Daraus ergibt sich folgender Versuch: Es sollen zwei nacheinander folgende Bewegungssequenzen á 2 Sekunden, die teilweise abgedeckt sind, den Kindern gezeigt werden und von innen als gleich erkannt werden. Während bei der ersten Bewegungssequenz ein Teil verdeckt wurde, war der gegenüberliegende Teil in der zweiten Sequenz verdeckt.

\section{Validierung der neuen Methode}

Für die Validierung muss die Methode einerseits daraufhin geprüft werden, ob Organisationsprinzipien in der Wahrnehmung und in der Vorstellung belegt werden können. Damit stellt sich die Frage, ob es in der Vorstellung nachweisbare mentale Ergänzungsleistungen gibt. Für die Feststellung der Kriteriumsvalidität ist andererseits eine Korrelationsstudie vorgesehen, die den Zusammenhang von Vorstellungen und Bewegungsfertigkeiten misst (siehe Kap. 8.3). 


\section{Hypothesen}

Es wurde hierbei angenommen, dass je größer das Bewegungswissen ist, desto größer ist auch die Wahrscheinlichkeit, dass die Aufgabe in dieser neuen Versuchsart gelöst wird. Das bedeutet, dass Lerner, die ein bestimmtes Wissen von Bewegungen schon im Langzeitgedächtnis gespeichert haben, diese auch für die Vorstellungsaufgabe nutzen können. Bewegungswissen erleichtert nach dieser These die Wahrnehmung und Vorstellung. Eine andere These wird aus der Studie von Ishai und Sagi (1997b) entnommen: demnach sollen Vorstellungen Wahrnehmungen erleichtern, wenn Vorstellungen aus dem Kurzzeitgedächtnis generiert werden. Wird das Langzeitgedächtnis aktiviert, dann stört die Vorstellung die Wahrnehmung. Übertragen auf diese Dissertation hieße dies, wenn die Kinder die gelernten Tennisbewegungen aus dem Langzeitgedächtnis abrufen (z.B. bei der Generierung von Vorstellungen), stören diese Vorstellungen die Wahrnehmung der Bewegungssequenzen (vgl. Kap. 7.2 für die ausführliche Darstellung der Studie von Ishai und Sagi (ebd.)). Die vorliegende Arbeit prüft die sich widersprechenden Hypothesen im Rahmen der Frage, welchen Einfluss Erfahrung auf das Wahrnehmen und Sich-Vorstellen von Bewegungssequenzen hat.

\section{Ergänzende Studien}

Ergänzend sollte der Einfluss der unterschiedlichen Trainingserfahrung auf die Wahrnehmungs- und Vorstellungsleistung ermittelt werden (siehe Kap. 8.7). Auch der Einfluss der Trainingserfahrung und des Geschlechts auf das Bewegungslernen (hier Gesamtskala des „Fragebogens zu Beherrschung der Tennistechnik“) soll hier beschrieben werden (siehe Kap. 8.8 und Kap. 8.9). 


\subsection{Fragestellungen im Überblick}

Die folgenden Fragen stehen im Kontext des Bewegungslernens in der Kindheit:

1) Welche Zusammenhänge bestehen zwischen dem Bewegungslernen und dem Wahrnehmen und dem Sich-Vorstellen von Bewegungen? (Studie 1)

2) Welchen Einfluss hat die Erfahrung auf das Wahrnehmen und das Sich-Vorstellen von Bewegungen? (Studie 2)

3) Welche Rolle spielen Geschlechterunterschiede hinsichtlich der Wahrnehmung und der Vorstellung von Bewegungen? (Studie 3)

4) Welche Rolle spielt die Versuchsbedingung hinsichtlich der Wahrnehmung und der Vorstellung von Bewegungen? (Studie 4)

5) Welche Rolle spielt die unterschiedliche Trainingserfahrung in der Versuchsgruppe für die Wahrnehmungs- und Vorstellungsleistung? (Studie 5)

6) Welche Rolle spielt die unterschiedliche Trainingserfahrung in der Versuchsgruppe für Veränderungen im Bewegungslernen? (Studie 6)

7) Welchen Einfluss hat das Geschlecht für Veränderungen im Bewegungslernen? (Studie 7) 


\subsection{Vorgehensweise}

\section{Theoretischer Teil}

Es werden im theoretischen Teil folgende Themen behandelt: der Wahrnehmungs- und Vorstellungsbegriff, verschiedene Messmethoden, verschiedene Modelle des Vorstellungsprozesses sowie ein Vergleich von Wahrnehmung und Vorstellung. Außerdem wird der Stand der Forschung erarbeitet und kritisch bewertet.

\section{Empirischer Teil}

\section{Voruntersuchungen}

Es werden eine Reihe von Voruntersuchungen mit den Kindern der Versuchsgruppe durchgeführt, z.B. der Kasten-Bumerang-Lauf. Dieser geht als Kovariate in die letzten beiden Studien (siehe Kap. 8.8 und Kap. 8.9) ein, da angenommen werden muss, dass bereits vor Beginn der Untersuchung bestehende Unterschiede in der Koordinationsfähigkeit Einfluss auf das Bewegungslernen haben. Andere Kovariaten sind die Anzahl der ausgeübten Sportarten, das Verhältnis von bewegungsnahen zu bewegungsfernen Aktivitäten und die geleisteten Trainingstage.

\section{Studie 1}

Um den möglichen Zusammenhang der Vorstellung mit dem Bewegungslernen zu messen, wurde eine Korrelationsstudie durchgeführt. Hierfür standen allein die Daten der Versuchsgruppe zur Verfügung. Dabei wurden zu verschiedenen Messzeitpunkten Bewegungsfertigkeiten anhand eines "Fragebogens zur Erfassung der Tennistechnik“ (siehe Anhang 9.1) bewertet. Per Videoanalyse haben lizenzierte Tennistrainer die Kinder bewertet und die anschließend ausgewerteten Daten wurden dann als Indikator für die Beherrschung von Bewegungsfertigkeiten und schließlich für das Bewegungslernen ausgewertet.

Die Bewegungsfertigkeiten wurden dabei anhand der Werte in der Gesamtskala und den Teilskalen des Fragebogens festgehalten. Diese Werte wurden mit Werten der Wahrnehmungs- und Vorstellungsaufgaben korreliert.

\section{Studie 2}

Um den Einfluss der Erfahrung auf die Wahrnehmung und Vorstellung von Bewegungen zu messen, wurde ein Gruppenvergleich durchgeführt. Untrainierte Kinder (Novizen), welche mit dem Tennisspiel nicht vertraut waren, wurden mit den Kindern verglichen, die das Tennisspiel während des Projekts „Jüngsten-Tennis“ erlernten. Diese Lernanfänger 
werden hier auch als Experten bezeichnet, da sie die „Expertise“ während des Projektes erwarben. Vergleichsmaßstab war die Leistung bei der mentalen Ergänzungsaufgabe, die im Kap. 8.3.2 detaillierter vorgestellt wird.

\section{Studie 3}

Um den Einfluss des Geschlechts auf die Wahrnehmung und Vorstellung von Bewegungen zu messen, wurden Jungen und Mädchen der Kontroll- und Versuchsgruppe miteinander verglichen. Vergleichsmaßstab war die Leistung bei der mentalen Ergänzungsaufgabe.

Studie 4

Hier wurde der Einfluss der Versuchsbedingung auf die Wahrnehmung und Vorstellung von Bewegungen gemessen.

\section{Studien 5 bis 7}

Zum Ausschluss von Alternativerklärungen wurde der Einfluss der Trainingserfahrung in der Versuchsgruppe sowohl auf die Vorstellungs- und Wahrnehmungsleistung als auch auf das Bewegungslernen untersucht. Die Versuchsgruppe bestand aus zwei unterschiedlich beschulten Gruppen, die ein für ihr jeweiliges Alter abgestimmtes Training erfuhren. Schließlich wurde der Einfluss des Geschlechts auf das Bewegungslernen ausgewertet. Inwieweit die zu Beginn vorhandenen Unterschiede in der Versuchsgruppe für spätere Ergebnisse verantwortlich waren, sollte anhand einer Kovarianzanalyse gemessen werden. Die Variablen Koordinationsfähigkeit (die zu Beginn der Untersuchung anhand des Kasten-Bumerang-Laufs gemessen wurde), die Anzahl ausgeübter Sportarten, die Trainingstage in Prozent sowie das Verhältnis von bewegungsnahen zu bewegungsfernen Aktivitäten wurden so auf ihren Einfluss auf das Bewegungslernen getestet. 


\section{Wahrnehmung}

\subsection{Begriff „Wahrnehmung“}

Die Vielfalt der theoretischen und philosophischen Auffassungen macht die genaue Begriffsbestimmung schwierig. Die Suche nach einer geeigneten Definition von Wahrnehmung im Standardwerk der Psychologie, der Enzyklopädie der Psychologie von Prinz und Bridgeman aus dem Jahr 1994, führte nicht zu einem befriedigenden Ergebnis. Beide Autoren geben folgende Definition an: „Perception has to do with the input side of the organism, with certain short-term consequences ... of variations in stimulating conditions"(Attneave, 1962, S. 620, zit. nach Prinz \& Bridgeman, 1994, S. 3).

Diese Definition ist allerdings sehr unbestimmt. Es wird deutlich, dass zum damaligen Zeitpunkt die Zusammenhänge zwischen Wahrnehmung und Handlung noch nicht im Fokus des Interesses standen. Da Wahrnehmungsvorgänge und Handlungsprozesse sehr ähnlich sind, wie später noch zu zeigen ist (siehe Kap. 6.1.3 zur funktionellen Äquivalenz), bedarf es auch einer Definition, die diese integriert.

Der Begriff der Wahrnehmung bezieht höhere Organisationsprozesse und Interpretationen der Sinnesempfindungen mit ein (Wilkening \& Krist, 1995). Die heutige Wahrnehmungsforschung hat nach Wentura und Frings (2013) zwei Aufgaben: zu ergründen wie eine Mentale Repräsentation von unserer Umwelt erstellt wird und wie diese unser Handeln in dieser Umwelt leitet (vgl. Kap. 5.4.1.1 für Begriffserklärung Mentale Repräsentation). Unter Wahrnehmung wird daher von Sekuler und Blake (2002) folgendes verstanden: „,...] acquisition and processing of sensory information in order to see, hear, taste, or feel objects in the world and it also guides an organisms actions with respect to those objects" (Sekuler \& Blake, 2002, S. 621, zit. nach Eysenck, 2012, S. 31).

Wahrnehmung hat in ihrer heutigen Auffassung demzufolge immer einen Bezug zur Handlung (vgl. auch die „common-coding“ Theorie von Prinz (1997) und die Theorie der Ereigniskodierung von Hommel, Müsseler, Aschersleben und Prinz (2001)).

Im Folgenden wird das Modell der Wahrnehmung von Milner und Goodale dargestellt. Später untersuchte Vorstellungsmodelle (siehe Kosslyn, 1994) weisen hierzu einen Bezug auf (vgl. Kap. 5.5). 


\subsection{Modell von Milner und Goodale}

Das Modell von Milner und Goodale (2004) versucht neuroanatomische Grundlagen der visuellen Wahrnehmung abzubilden. Aufgrund von Befunden zu Läsionsstudien bei Tieren wurden erstmals zwei visuelle Systeme von Ungerleider und Mishkin (z.B. Mishkin, Ungerleider \& Macko, 1983) unterschieden: eines für die Objektwahrnehmung, ein anderes für die Wahrnehmung des Raumes. Milner und Goodale (1995, 1998, 2008, zit. nach Eysenk, 2011) und Goodale und Milner (2004) haben diese zwei Arten von visuellen Systemen spezifiziert:

a) Das „vision-for-perception“ System sei für die Verarbeitung von Formen wichtig. Die Informationsverarbeitung geschehe im ventralen Pfad. Es transformiere die visuelle Information in Wahrnehmungsrepräsentationen von Objekten und ihren Relationen. Dann werde innen Bedeutungen zugewiesen, die Kausalbeziehungen ergründet und Wissen generiert. Hier geschehe das bewusste Erkennen. Das ventrale System wird "what"-System genannt.

b) Das „vision-for-action“ System sei für die Verarbeitung der relativen Position zu einem Objekt verantwortlich (dorsaler Pfad). Dieses System sei für die visuelle Kontrolle objektgerichteter und die Ausführung zielgerichteter Handlungen verantwortlich. Das dorsale System wird als das "where"-System und von Bertenthal (1996) auch "how"-System bezeichnet.

Beide Systeme haben ihren Ursprung in der primären Sehrinde (auch Areal V1 genannt) (Gazzaniga et al., 2009). Die primäre Sehrinde erhält als erstes Informationen über die Augen (Kosslyn \& Thompson, 2003). Dabei ist das Langzeitgedächtnis für Formen im inferioren Temporallappen repräsentiert, räumliche Repräsentationen hingegen werden im posterioren Parietalkortex gespeichert (Eysenck, 2012).

Kritisch ist zu bewerten, dass die Befunde aus Läsionsstudien gewonnen werden. Diese sind Einzelfallstudien an Tieren oder Patienten mit Verletzungen des Gehirns. Hier können keine Kausalfaktoren und allgemeine Zusammenhänge aufgedeckt werden. Außerdem spielt bei Läsionen immer die Plastizität des Gehirns eine Rolle. So werden Ausfälle durch andere Gebiete im Gehirn kompensiert. Auch sind Vergleiche von Läsionen bei Tieren mit der menschlichen Anatomie problematisch. 


\subsection{Organisationsprinzipien der Wahrnehmung}

Mit den Gestaltgesetzen der Wahrnehmung haben sich z.B. die Gestaltpsychologen Wertheimer (1923, 1925), Köhler (1929) und Koffka (1935) befasst. Nach ihrer Auffassung gehören die Gestaltgesetze zu den Organisationsprinzipien der Wahrnehmung, deren Inhalt die Gliederung von Wahrnehmungsinhalten zu Formen und Figuren sind. Dabei werden beispielsweise in der Nähe liegende oder gleiche Objekte als Gruppierung aufgefasst (Gesetz der Nähe, Gesetz der Gleichheit) oder Figuren mit durchbrochenen Linien werden als ganze Figur gesehen (Gesetz der Geschlossenheit). Das Gesetz der guten Fortsetzung besagt, dass etwa bei sich kreuzenden Linien das wahrgenommen und erkannt wird, was eine Figur ergibt (wie z.B. einen Kreis oder ein Trapez). Rosenzweig et al. (1999) weisen übrigens darauf hin, dass die Wahrnehmung der "illusory contours“, also der Kanten, die nicht gezeichnet sind, in einer frühen Stufe des visuellen Systems verarbeitet werden. Dies geschieht im sogenannten Areal V2, der sekundären Sehrinde, welches an das Areal V1, die primäre Sehrinde, angehängt ist.

Die oben genannten Beispiele sind alle statischer Natur. Dynamische Bilderfolgen, welche unvollständig präsentiert werden, könnten mittels mentaler Simulation ebenfalls ergänzt werden. In dieser Dissertation sollen die Kinder Videoaufnahmen, die während inrer Präsentation teilweise abgedeckt sind, mental zu einer vollständigen Bildfolge ergänzen. Dabei sind die oben genannten Gestaltgesetze nur bedingt geeignet, um auf diese Situation angewendet zu werden. Sie sind somit nur ein gedankliches Hilfsmittel für eine Beschreibung der Ergänzungsleistung, denn die Gestaltgesetze beschreiben eher und erklären weniger. Für Buether (2010) liegt die Ursache einer visuell-räumlichen Kompetenz nicht in den Gestaltgesetzen, sondern in der „multisensuellen Auseinandersetzung mit der Umwelt“ und im Gebrauch der Sprache mit dem sich dadurch entwickelnden Zeichensystem. Buether (ebd.) schreibt:

„Die 'gute Gestalt' ist daher das Ergebnis eines Ästhetisierungsprozesses, welcher der soziokulturellen Anpassung der natürlichen Umwelt an die Anforderungen des zwischenmenschlichen Zusammenlebens folgt und dem Bedürfnis nach Regeln Ausdruck gibt, über welche die Umgestaltung der Natur kontrolliert und in Übereinstimmung gesellschaftlicher Anschauungen erfolgen kann." (Buether, 2010, S.27)

Zusammenfassend gilt demnach, dass die mentale Ergänzung von dynamischen Bildfolgen mit den Gestaltgesetzen nur eingeschränkt beschrieben werden kann. 


\subsection{Geschlechterunterschiede in der Wahrnehmung}

Kimura (1999) fasst Studien zu Geschlechterunterschieden in der Wahrnehmung zusammen. Hiernach schneiden Frauen besser ab, wenn es um die Erfassung von Tiefe im interpersonalen Raum geht, zum Beispiel können sie besser Gesichtsausdrücke und nonverbale Reize entschlüsseln. Auch haben Frauen ein größeres visuelles Feld (Burg, 1968). Das bedeutet, wenn Frauen auf einen Reiz in der Mitte sehen sollen, können sie Gegenstände, die weiter weg sind noch in größerem Abstand wahrnehmen als Männer. Männer hingegen haben eine größere Genauigkeit, so dass sie schmälere Objekte im visuellen Feld wahrnehmen können (Burg, 1966). Männer können leuchtende Objekte, die schnell abwechselnd aufleuchten, besser wahrnehmen als Frauen (Ginsburg et al., 1982). Auch gibt es geringe Geschlechterunterschiede beim stereoskopischen (räumlichen) Sehen. Dies ist allerdings durch den unterschiedlich großen Augenabstand bedingt. Frauen geben weiterhin genauere Antworten und identifizieren die Testreize (,random dot pattern”) schneller als Männer. Außerdem gibt es Vorteile bei Frauen bei in der Wahrnehmungsgeschwindigkeit (Kimura, 1999). So können sie, etwa im ZahlenSymbol-Test des Intelligenztestes von Wechsler (Wechsler, 1958), die Zahlen schneller zu den abstrakten Symbolen zuordnen. Die Wahrnehmung von Frauen soll wiederum "feldabhängiger" sein, das heißt, sie werden mehr von Umweltreizen beeinflusst, die in ihr Urteil mit eingehen (Witkin, 1967).

Im Kapitel 7.7 sind die Geschlechterunterschiede bei Vorstellungsleistungen dargestellt. 


\section{Vorstellung}

Die Heterogenität des Vorstellungskonstrukts schlägt sich in verschiedenen theoretischen Konzeptionen und Operationalisierungen nieder. Es folgen deshalb eine Begriffsbestimmung mit Einordnung bezüglich anderer Konstrukte und die Darstellung des eigenen Konstrukts. Anschließend werden die Funktionen von Vorstellungen, die Kurzzusammenfassung der wichtigsten Messverfahren mit kritischer Einschätzung sowie die Eigenschaften von Vorstellungen erläutert.

\subsection{Begriff „Vorstellung“}

Vorstellungen haben den Charakter eines Erlebnisses (Bosshardt, 1973). Vorstellungen können auch als kognitive Repräsentationen einer (komplexen) motorischen Fertigkeit aufgefasst werden (Reed, 2002). Allgemein kann gesagt werden, dass mentale Vorstellungen eine Sammlung verschiedenster Fähigkeiten sind (siehe dazu auch Kosslyn, Ganis \& Thompson, 2010).

Die „Stanford Encyclopedia of Philosophy“ definiert mentale Vorstellungen wie folgt: „Mental imagery (varieties of which are sometimes colloquially referred to as "visualizing," "seeing in the mind's eye," "hearing in the head," "imagining the feel of" etc.) is quasi-perceptual experience; it resembles perceptual experience, but occurs in the absence of the appropriate external stimuli. [...] Very often these experiences are understood by their subjects as echoes or reconstructions of actual perceptual experiences from their past; at other times they may seem to anticipate possible, often desired or feared, future experiences." (Thomas, 2010)

Mit diesem letzten Aspekt sind auch Prozesse wie die Antizipation eingeschlossen. Die Zielhandlungen müssen beim Erlernen der Bewegung vorgestellt werden, um sie anschließend zu reproduzieren oder die Tennisspieler müssen die Ballposition aufgrund der Körperstellungen des Gegners antizipieren.

\subsubsection{Einordnung zu anderen Fähigkeiten}

Kohen (1954) bildet die „Wahrnehmungsnähe“ von Vorstellungen auf einem Kontinuum ab: sie seien auf einem Pol „realitätsnah“, auf dem anderen Pol „phantasienah“. Während "Gedächtnisvorstellungen“ und „Anschauungsbilder“ nahe des ersten Pols verankert sind, sind es die „Phantasievorstellungen“ nahe des zweiten Pols. 
„Bewegungsvorstellungen“ werden neben „Gegenständlichen Vorstellungen“ und „autonomen realisierbaren Realvorstellungen“ zwischen beiden Polen angesiedelt. Dies geht mit der Definition von Thomas (2010) konform: Vorstellungen werden sowohl als Antizipationen der Zukunft, als auch als Reproduktion der Vergangenheit konzipiert. Jeannerod (2001) ordnet die motorischen Vorstellungen als „s-states“ zu Phänomenen wie: intendierte Handlungen, prospektive Handlungsurteile, wahrnehmungsbasierte Entscheidungen, beobachtete Handlungen Dritter und Handlungen im Traum.

Für Golledge (1999) etwa gehören mentale Vorstellungen zu den visuo-räumlichen Fähigkeiten, wobei als weitere Beispiele hierfür die Visualisierung, die Mentale Rotation (siehe Kap. 5.3.2 für die Begriffserklärung) das visuell-räumliche Gedächtnis, die räumliche Perspektivenübernahme, die Orientierung und das Finden eines Weges sowie die Interpretation von Karten gelten.

Gegen diese Zuordnung sprechen Befunde von Munzert (2001a), die eine geringe Korrelation zwischen mentalen Vorstellungen und Daten von Leistungstests, die räumliche Fähigkeiten erfordern, aufweisen.

\subsubsection{Arten von Vorstellungen}

Es werden meist visuelle und motorische Vorstellungen unterschieden, obwohl die theoretischen Auffassungen dazu widersprüchlich sind. Hier sollen zunächst zwei Arbeitsdefinitionen dargestellt werden:

1) Die visuelle Vorstellung wird definiert durch: „Visual imagery, by it's very nature, involves the representation of the spatial components of the perceived world" (Stevens, 2005, S. 330).

Allerdings ist diese Definition unzureichend, da sie sich nur auf den dorsalen Pfad der Informationsverarbeitung bezieht (vgl. das Modell der Vorstellungen von Kosslyn in Kap. 5.5 mit dem Modell von Milner \& Goodale in Kap. 4.2). Aufgrund der These der Ähnlichkeit zwischen Wahrnehmung und Vorstellung (Kap. 6.1) sowie den neuroanatomischen Gesichtspunkten zur Wahrnehmung (Kap. 4.2) wird zudem davon ausgegangen, dass visuelle Vorstellungen nicht nur räumliche Aspekte, sondern auch Farben und Formen beinhalten können. Belege hierfür liefern z.B. Kosslyn, Ganis und Thompson (2010). Komponenten mentaler Vorstellungen können unabhängig voneinander gestört sein: während sich einige Patienten Form und Farbe noch vorstellen können, kann bei anderen Patienten die Mentalen Rotation von Objekten selektiv gestört sein und vice versa.

Deshalb heißt es allgemeiner in einer neueren Definition von Ishai (2010): „Visual 
imagery is the ability to generate percept-like images in the absence of retinal input and is therefore a vivid demonstration of retrieving pictorial information in memory" (Ishai, 2010, S.1 bezüglich des Online-Dokuments). Wobei hier Ishai (ebd.) das Repräsentationsformat im Sinne Kosslyns als bildlich auffasst (siehe Kap. 5.4.1).

2) Unter der motorischen Vorstellung wird verstanden: „In the most general sense, motor imagery refers to the 'mental rehearsal of simple or complex motor acts that is not accompanied by overt body movements'(Jeannerod, 1995; Porro et al., 1996)“ (Solodkin et al., 2004, S. 1246).

Naito (1994) unterscheidet zwei Varianten von Vorstellungen: eine beobachtungsbezogene und eine körperzentrierte Variante. Meist ist unter dem ersten Begriff die visuelle Vorstellung (in der Dritte-Person-Perspektive) zu verstehen und unter dem zweiten Konzept die motorische Vorstellung (in der Erste-Person-Perspektive). Munzert et al. (2009) weisen diesbezüglich darauf hin, dass Experten, zum Beispiel Musiker oder Slalomfahrer, mehrere Arten von Vorstellungsbildern nutzen würden und problemlos zwischen verschiedenen Perspektiven (Erste- vs. Dritte-Person-Perspektive) hin- und herschalten.

Solodkin et al. (2004) referieren Studien, aus deren Ergebnis die Bewegungsvorstellung in eine visuelle und eine kinästhetische Komponente unterteilt wird. Dabei werden die Funktionen „motorische Vorbereitung“, „Imitation“, „Antizipation“ und "Verfeinern motorischer Fertigkeiten“ eher dem kinästhetischen Teil zugeordnet. Das zeigt die Nähe, in die das Konzept motorischer Vorstellung zur Handlungsbeobachtung und Handlungsausführung gebracht wird.

Es werden zwar von den meisten Autoren visuelle von motorischen Vorstellungen unterschieden, dabei sind diese nicht immer klar voneinander zu trennen: So wird angenommen, dass Bewegungsvorstellungen sowohl in visuellen, auditiven als auch propriozeptiven Repräsentationsformaten gespeichert sein können (Wiemeyer, 2001).

Munzert und Reiser (2003) betonen, dass je nach Aufgabe beide Facetten mit unterschiedlicher Gewichtung integriert werden. Auch andere Autoren weisen darauf hin, dass motorische Vorstellungen möglicherweise zusätzlich zu den motorischen oder kinästhetischen Anteilen (Annett, 1995, zit. nach Smyth \& Waller, 1998) auch visuellräumliche Anteile enthalten (Elliott \& Maraj, 1994; Jackendoff \& Landau, 1994; Klatzky, 1994, zit. nach Smyth \& Waller, 1998).

Auch für Jeannerod (1994) können motorische Vorstellungen nicht ganz von visuellen Vorstellungen getrennt werden. Hingegen sind Kosslyn, Ganis und Thompson (2001) der 
Auffassung, dass motorische und visuelle Vorstellungen als jeweils eigenständiger Mechanismus bestehen, wenn auch motorische häufig von visuellen Vorstellungen begleitet werden.

\subsubsection{Konzepte zu Vorstellungsfähigkeiten}

Lequerica et al. (2002) unterscheiden beispielsweise folgende drei Arten von Vorstellungsfähigkeiten: Vorstellungskontrolle (imagery control), Vorstellungsgenerierung (imagery generation) und Vorstellungslebendigkeit (imagery vividness). Unter Vorstellungskontrolle wird die Fähigkeit verstanden, Mentale Repräsentationen zu verändern (Richardson, 1977, zit. nach Lequerica et al., 2002). Mit Vorstellungsgeneration ist die Erzeugung und Formung von mentalen Vorstellungsbildern ohne externe visuelle Reize gemeint (Dean \& Morris, 1991, zit. nach Lequerica et al., 2002), während die Vorstellungslebendigkeit die Klarheit, Helligkeit oder Intensität der Mentalen Repräsentationen bezeichnet (Marks, 1973, zit. nach Lequerica et al., 2002).

Dieses Konzept von Vorstellungen wird durch Fragebögen gemessen. Diesbezügliche Nachteile werden in Kap. 5.3.1 dargestellt.

In dieser Dissertation wird ein alternatives Konzept verfolgt: Es werden Vorstellungen als mentale Ergänzungsleistungen verstanden, denn Vorstellungen werden als die mentale Simulation von Handlungen aufgefasst (Jeannerod, 2001). Dabei sollen in der Vorstellung Bewegungssequenzen, die nicht sichtbar sind, aus gleichzeitig sichtbaren Bewegungssequenzen konstruiert werden. So werden Vorstellungen als wahrnehmungsgebunden konzipiert. Dies stellt eine Modifikation des Konzepts der Vorstellungsgenerierung von Lequerica et al. (2002) dar.

Dabei spielen womöglich gespeicherte Erfahrungen aus dem Langzeitgedächtnis ebenso eine Rolle, wie Repräsentationen von Handlungen, die im Kurzzeitgedächtnis aktiviert werden (zum Repräsentationsbegriff siehe Kap. 5.4.1.1). 


\subsection{Funktionen von Vorstellungen}

Vorstellungen werden hier in dieser Arbeit als das Tor zum Bewegungslernen angesehen. So bieten sie einen Zugang zu Bewegungsrepräsentationen, d.h. Wissen, das beim motorischen Lernen gebildet wird und für die motorische Kontrolle notwendig ist (Munzert et al., 2009).

Funktionen von motorischen Vorstellungen sind vielfältig. Es wird angenommen, dass motorische Vorstellungen etwa für folgende Bereiche gebraucht werden:

- für die Handlungsplanung (Jeannerod, 2006; Prinz, 1994)

- für die soziale Kognition (z.B. das Erkennen von Intentionen anderer, die Vorhersage von Handlungen, das Erkennen von Emotionen sowie das Sprachverstehen) (Jeannerod, 2006)

- mental updating: die Antizipation einer visuellen Konsequenz einer Bewegung (Hirsch et al., 2003)

- Bewegungslernen (Kosslyn, Behrmann \& Jeannerod, 1995).

Mentale Vorstellungen dienen als Modelle von Handlungszielen (Hacker, 1998) und besitzen somit eine handlungsregulierende Funktion. Die Bewegungsvorstellungen ist für alle drei Phasen der Handlungsregulation bedeutend (Munzert, 2001a): für die Vorbereitung der Handlung, die Handlungsrealisation und die Handlungsinterpretation. Jeannerod $(1994,2001)$ und andere betonen die Funktion von motorischer Vorstellung im Sinne der Handlungsplanung: "Motor imagery has been described as the class of image of one's own bodily movements which are used to stimulate or plan for subsequent action" (zit. nach Stevens, 2005, S. 330).

Es wird angenommen, dass Bewegungsvorstellungen eine große Bedeutung für das Lernen von Bewegungen haben, sei es beim Fertigkeitserwerb oder in der Wiederherstellung bereits erworbener Fertigkeiten: "There is now repeated evidence that motor imagery has significant positive effects on motor skill learning" (Denis, 1985; Feltz \& Landers, 1983, zit. nach Decety, 1996). Durch den inneren Vollzug der Bewegung können Vorstellungen für das Lernen von Bewegungen förderlich sein: Es wurde gezeigt, dass sich Geschwindigkeit, Genauigkeit, Kraft und Zuverlässigkeit der Bewegungsdurchführung durch Vorstellungsprozesse ändern (Feltz \& Landers, 1983; Yue \& Cole, 1992).

Wiemann (2001) versteht Bewegungsvorstellungen auch als kreatives Element: Durch den Vorstellungsprozess erfolge auch die Neukombination von Bewegungen. 


\subsection{Untersuchungsverfahren zur Messung von Vorstellungen}

Munzert (2001a) unterscheidet zwei Hauptrichtungen der Methodik: die phänomenologische Herangehensweise basierend auf „subjektiver“ Skalierung von Vorstellungen und die der funktionalen mittels „objektiver" Leistungstests, wobei das Anführungszeichen andeutet, dass auch hier subjektive Elemente beinhaltet sind, wie etwa der Einfluss der verschiedenen Auswerter. Hier werden zusätzlich noch die neurophysiologischen Methoden als spezielle Form funktionaler Tests ergänzt.

\subsubsection{Fragebögen}

Mit dem Fragebogen VVIQ (Vividness of Visual Imagery Questionnaire, Marks, 1973) wird auf einer unipolaren fünfstufigen Skala die Lebhaftigkeit von Vorstellungen bewertet. Dabei sollen verschiedene Szenarien vorgestellt werden, z.B. eine Person, ein Naturschauspiel, ein Gebäude oder eine Landschaft, welche dann selbst hinsichtlich der Klarheit der Vorstellungsbilder eingeschätzt werden. Allerdings wurden keine Korrelationen zu räumlichen Fähigkeiten gefunden (z.B. Dean \& Morris, 2003). Zudem sind unipolare Ratingskalen unpräziser als bipolare (Bortz \& Döring, 2006). Das bedeutet hier, dass das Konstrukt Lebhaftigkeit der Vorstellungen möglicherweise nicht klar genug definiert wird. Es handelt sich zudem um allgemeine visuelle Vorstellungsbilder.

Beim Fragebogen VMIQ (Vividness of Movement Imagery Questionnaire, Isaac et al., 1986) werden Vorstellungen von Bewegungen aus dem Altag in internaler und externaler Perspektive hinsichtlich der Lebendigkeit bewertet. Dabei steht zum einen die internale Perspektive für die Innensicht, aus der die eigene Bewegung wahrgenommen und vorgestellt wird. Damit ist die kinästhetische Vorstellung gemeint. Zum anderen gibt es die externale Perspektive, diese ist die Außensicht, wonach eine andere Person die vorgestellte Bewegung ausführt: Sie ist demnach die visuelle Vorstellung. Allerdings ist das Verständnis dieser Items bei Kindern von bis zu 10 Jahren stark eingeschränkt (Livesey, 2002).

Beim MIQ-Fragebogen (Movement Imagery Questionnaire, Hall \& Martin, 1997; Hall, Pongrac \& Buckolz, 1985, zit. nach Munzert, 2001b) werden Bewegungen ausgeführt, die vorgestellt und beurteilt werden sollen. Der MIQ misst demnach sowohl visuelle als auch kinästhetische Vorstellungen. 
Die beiden letzten Fragebögen unterscheiden sich darin, ob auf das Langzeitgedächtnis (VMIQ) oder das Arbeitsgedächtnis (MIQ) zurückgegriffen werden muss (Munzert, 1996).

Der MIQ-Fragebogen wird von Dültgen, Munzert und Möllmann (1996) wegen der geringen Aufgabenschwierigkeit, schlechter Aufgabenanalyse und den unspezifischen Bewegungsaufgaben kritisiert. Weiterhin haben Fragebögen im Allgemeinen den Nachteil, dass sie bei Kindern ein bestimmtes sprachliches Verständnis sowie Ausdrucksvermögen voraussetzen, zudem sind mentale Vorgänge sprachlich oft nicht beschreibbar. Oft, so Dültgen et al. (1996), wird auch unzulässigerweise davon ausgegangen, dass die Probanden bei der Beantwortung der Aufgaben dieselben Konstrukte zugrunde legen. Die Lebendigkeit von Vorstellungen stellte sich bei ihrer Untersuchung als nicht vordergründig heraus. Das bedeutet, die Vorstellungsbilder wurden als vollständig empfunden, obwohl sie nur bestimmte Aspekte wiedergaben. Zentral waren hingegen Merkmale wie "Veränderbarkeit“ und „Kontrolle“. Letzteres hatte zum einen inhaltliche Bezüge (Kann die Vorstellung nach Zielvorgaben entwickelt werden?) zum anderen zeitliche (Kann die Vorstellung in zeitlicher Nähe zur Aufgabenstellung erzeugt werden?).

\subsubsection{Mentale Rotation}

Die Mentale Rotation ist ein Verfahren, was auf der mentalen Drehung von zwei- oder dreidimensionalen Figuren basiert, die jeweils von ihrer Originalposition in einem bestimmten Rotationswinkel abweichen oder in der Kontrollbedingung spiegelbildlich verwendet werden. Shepard und Metzler (1971) haben dieses Verfahren entwickelt. Das Reizmaterial hat jedoch wenig mit der Wahrnehmung biologischer Bewegungsabläufe zu tun, wie sie im Sport eine Rolle spielen. Neben den schon in der Einleitung angesprochenen Nachteilen der Methode der Mentalen Rotation, gibt es weitere Argumente gegen den Einsatz bei Kindern: Einerseits entwickeln Kinder schon ab 4 Jahren die Fähigkeit zur Mentalen Rotation; die Leistungen der Kinder sind aber sehr heterogen (Estes, 1998). Andererseits sind die Rotationsgeschwindigkeiten bei jüngeren Kindern kleiner und die Fehlerraten werden mit zunehmendem Rotationswinkel bei 5jährigen Kindern größer. Das bedeutet je mehr die Figur gedreht ist, desto schwerer haben es Kinder, diese Figur mit der Vergleichsfigur abzugleichen. Es wurde bei Kosslyn, Margolis, Barrett, Goldknopf und Daly (1990) allerdings kein GeschlechterEffekt zu den abhängigen Variablen (Rotationsgeschwindigkeit und Fehlerrate) ermittelt. Heterogene und verminderte Leistungsfähigkeit bei jüngeren Kindern (4 und 5 Jahre) 
sowie fehlende kindliche Geschlechterdifferenzen beim Test für Mentale Rotation bedeuten, dass der Test der Mentalen Rotation hinsichtlich des Alters, nicht aber hinsichtlich des Geschlechts, differenzieren kann. Ob die Ergebnisse hinsichtlich des fehlenden Geschlechtereffekts bei Kosslyn et al. (ebd.) aufgrund von gleichen oder verschiedenen oder gleich guten Strategien der Kinder auftraten, konnte so nicht ergründet werden.

\subsubsection{Mentale Chronometrie}

Die Dauer von vorgestellten Handlungen wird bei diesem Verfahren mit der Dauer von tatsächlich ausgeführten Handlungen bzw. von beobachteten Handlungen verglichen (z.B. Caeyenberghs et al., 2009b).

Das Verfahren betont ausschließlich zeitliche Aspekte der Prozesse des SichVorstellens, Wahrnehmens und der Handlungsausführung. Diese zeitlichen Vergleiche sind zudem undifferenzierter als bei anderen Verfahren (siehe Wiemeyer und Angert (2011) in Kap. 5.3.5).

\subsubsection{Interviews}

Das Interview zielt darauf ab, Vorstellungsmodi qualitativ zu erfassen. Dültgen, Munzert und Möllmann (1996, zit. nach Munzert, 2001b) haben Interviews bei 10- bis 17-jährigen Badminton-Spielern durchgeführt. Sie benutzten konkrete Vorstellungsaufgaben und werteten das Leitfaden-Interview zu folgenden Fragen aus: Wie lebendig sind die Vorstellungen? Aus welcher Perspektive werden sie erzeugt? In welcher zeitlichen Abfolge und wie veränderbar konnte die Aufgabe vorgestellt werden?

Bei dieser Methode muss der Interviewer genau darauf achten, ob er den Interviewpartner noch versteht (Dültgen et al., 1996).

Die Gefahr besteht zudem darin, dass das Ziel dieser sogenannten „störungsfreien“ Kommunikation nicht immer erreicht wird. Dültgen et al. (ebd.) nennen als Beispiel hierfür sozial erwünschte Antworten, den Bezug auf allgemeine Erfahrungen und Vorstellungen, die nicht konkret genug ausfallen. In dieser Studie wurden 7 von 42 Interviews ausgeschlossen, weil sie diese Störungen enthielten. Auch fehlendes metakognitives Denkvermögen, also die Art und Weise, wie über das eigene Denken und Sich-Vorstellen nachgedacht wird, ist ein weiterer Grund für die Kommunikationsprobleme (Dültgen et al., 1996). Zudem sind Hilfestellungen und Anpassungen der Fragen an den Befragten zulässig, die die Vergleichbarkeit der Interviews beeinflussen. Als einzeln verwendete Methode sind Interviews nicht geeignet, 
Bezüge zu sportlichen Fertigkeiten aufzudecken, da hier das sprachliche Vermögen im Sinne von „Wie spreche ich über...?" im Vordergrund steht.

\subsubsection{Bilderergänzungstests}

Weiterhin sind die Bilderergänzungstests zu nennen, wobei ein Proband eine Reihe von unvollständigen statischen Bildern ordnet oder fehlende Bilder ergänzt (siehe Rockmann (1998) für einen manuellen Test und Wiemeyer und Angert (2011) für einen softwarebasierten Test dieser Art).

Diese Methode ist am ähnlichsten zu der Methode, die in dieser Dissertation verwendet wird. Im Vergleich zu Wiemeyer und Angert (2011) wird hier in der Dissertation besondere Aufmerksamkeit auf Fehlerraten gelegt, während erstere Reaktionszeiten und die dahinter liegenden Prozesse zur Konstruktion Mentaler Repräsentationen in Augenschein nehmen. Siehe auch das Konzept in Kap. 5.3.3, in welchem Reaktionszeiten und die damit verbundenen Prozesse im Vordergrund stehen. Der Vorteil der Angabe von Fehlerraten ist, dass durch sie angegeben werden kann, wie zuverlässig das psychische System funktioniert. Damit wird ein anderer Forschungsschwerpunkt gesetzt.

Das Problem bei Bilderergänzungstests wird wie folgt erläutert: Bei der Verwendung von statischem Reizmaterial besteht seitens des Versuchsteilnehmers die Möglichkeit, Standbilder an bestimmten Punkten im Bewegungsablauf zu bilden. Diese Standbilder haben eine besondere Bedeutung für den Verlauf der Bewegung, sind aber nicht dynamische Vorstellungen von der Bewegung im engeren Sinn. Mit dem hier verwendeten dynamischen Reizmaterial soll geprüft werden, ob Zusammenhänge auch für dieses Reizmaterial nachweisbar sind. Die Dissertation versteht sich diesbezüglich als methodische Komplettierung zum Bilderergänzungstest.

\subsubsection{Funktionelle Magnetresonanztomografie}

Die funktionelle Magnetresonanztomografie (fMRT) gehört zu den neurophysiologischen Methoden und basiert auf der Anregung der Wasserstoffatome im Blut. Je mehr Aktivität im Gehirn vorhanden ist, umso mehr Resonanz soll das Verfahren anzeigen. Es bleibt aber die Frage offen, ob mit diesem Verfahren zentralnervöse Prozesse bei Experten abgebildet werden können, denn bei innen sind womöglich diese, aufgrund der Automatisierung von Fertigkeiten, nicht mehr messbar.

Speziell das fMRT weist Mängel an Objektivität, Reliabilität und Validität auf (Bennett et al. 2009; Bennett \& Miller, 2010) und ist zudem sehr aufwendig in der Durchführung und 
Auswertung.

\subsubsection{Entwicklung einer alternativen Messmethode}

Um die Nachteile der genannten Verfahren auszugleichen, wird deshalb vorgeschlagen, ein alternatives Messverfahren zu entwickeln. Diese neue Methode dient als Ergänzung bereits bestehender Verfahren und wurde zur Untersuchung von Vorstellungen der Bewegungen in der Kindheit entwickelt. Sie basiert auf dem Prinzip mentaler Ergänzungen von dynamischem Reizmaterial.

Dabei sind in der Vorstellungsbedingung jeweils beim ersten Video eines Videopaares die eine und dann beim zweiten Video des gleichen Paares die zweite komplementäre Hälfte abgedeckt. In der Basisbedingung werden die Videopaare ohne Abdeckung gezeigt (Wahrnehmungsbedingung). Aufgabe ist dabei zu entscheiden, ob beide Videopaare gleich sind, unabhängig von der jeweiligen Abdeckung (siehe Abbildung 7 in Kap. 8.3.2.1).

Das in dieser Dissertation neu entwickelte Verfahren basiert auf der Annahme, dass je mehr Erfahrungswissen vorhanden ist, desto mehr gezeigte Paare von Videosequenzen als identisch identifiziert werden können. Das bedeutet, dass diejenigen Kinder hier besser abscheiden sollten, die motorische und visuelle Erfahrungen während eines Tennistrainings erworben haben, als solche Kinder, die diese Erfahrungen nicht haben.

Einer anderen Hypothese nach ist das Langzeitgedächtnis bei der Vorstellungsgeneration beteiligt und es kommt zu einer Erschwerung der Wahrnehmung (siehe Ishai \& Sagi, 1997b in Kap. 7.2).

In Abhängigkeit der Art des Gedächtnisses, das involviert ist, kommt es zu unterschiedlichen Effekten, wobei einerseits die Vorstellung die Wahrnehmung stört (Perky, 1910) und andererseits die Vorstellung die Wahrnehmung erleichtert (Ishai \& Sagi, 1997a). Dies haben Ishai und Sagi (1997b) in ihrer Studie untersucht. Sie fanden heraus, dass der Abruf visueller Informationen aus dem Langzeitgedächtnis (LZG) die Wahrnehmung störe. Hingegen werde die Reizerkennung erleichtert, wenn hierzu Informationen aus dem Kurzzeitgedächtnis (KZG) abgerufen werden (siehe Kap. 7.2).

Hingegen betonte Eysenck (2012), dass gleiche Inhalte bei Vorstellung und Wahrnehmung als Grund für die Erleichterungseffekte gelten und unterschiedliche Inhalte die Interferenzeffekte erklären (siehe Kap. 7.2). 


\subsection{Eigenschaften von Vorstellungen}

Im folgenden Abschnitt werden einige Merkmale und Eigenschaften von Vorstellungen dargestellt.

Laut Scherer (2003) können Vorstellungsinhalte emotional gefärbt sein und vegetative Erscheinungen aktivieren. Sie können visuelle, akustische oder kinästhetische Qualitäten enthalten.

\subsubsection{Repräsentationsformat von Vorstellungen: bildlich versus symbolisch}

In der sogenannten Imagery-Debatte geht es um das Format der Repräsentation von Vorstellungen. Dabei argumentierte Kosslyn (1980) für ein analoges Repräsentationsformat von Vorstellungen, Pylyshyn (1981) plädierte für ein abstraktes Format. Es soll deshalb zuerst der Begriff der Mentalen Repräsentation erläutert werden, da er für das Verständnis dieser Debatte von Bedeutung ist.

\subsubsection{Begriff Mentale Repräsentation}

Dazu können die Ausführungen von Palmer (1978) herangezogen werden. Für Palmer (ebd.) sind zunächst Repräsentationen etwas, was repräsentiert bzw. dargestellt wird. Dazu gehören sinngemäß bestimmte Aspekte der repräsentierten Welt und ein Medium, das bestimmte Aspekte der zu repräsentierenden Welt beinhaltet. Zwischen beiden Welten bestehen Korrespondenzen, also Relationen. Zudem gibt es Prozesse, die diese beiden Welten einander zuordnen. Es komme auf die Informationen an, nicht auf das Medium. Dennoch trägt Palmer (ebd.) etwas zur Debatte um das Format von Vorstellungen bei. Einer weniger starken Behauptung zufolge wird eine analoge Welt repräsentiert. Einer stärkeren Behauptung nach wird eine analoge Welt in einem analogen, räumlichen Medium repräsentiert. Palmer (ebd.) nennt dies einen „Isomorphismus 1. Ordnung“ oder auch physikalischen Isomorphismus. Dabei bedeutet „iso" „gleich“ und „morph“ meint „Form“. Ein „Isomorphismus 2. Ordnung" oder auch funktionaler Isomorphismus besteht, wenn etwa die Welt nicht im analogen Medium, sondern in einer abstrakteren Form repräsentiert wird.

\subsubsection{Ergebnisse der Debatte}

Dabei ist diese Debatte laut Opwis und Lüer nicht auflösbar, denn es stellt sich das Problem der "prinzipiellen Unbeobachtbarkeit jeglicher Repräsentationsformen im Gedächtnis" (Opwis \& Lüer, 1996, S. 398). Unter anderem wurde mit bildgebenden Verfahren versucht, dennoch eine Antwort auf diese Frage zu finden. Mast (2005) fasst 
dazu Studien zusammen. Genau genommen referiert Pylyshyn (2002) zu den Repräsentationsinhalten, während Kosslyn (1994) zu den Verarbeitungsprozessen Aussagen trifft. Dies würde bedeuten, dass diese Theorien sich nicht gegenseitig ausschließen.

\subsubsection{Sind Vorstellungen bewusst?}

Vorstellungen von Bewegungen werden gemeinhin als bewusste Einheiten konzeptioniert (z.B. Daugs \& Blischke, 1984; Jeannerod, 2006; Richardson, 1969), die in Abwesenheit von Reizen in Erscheinung treten. So definiert Richardson (1969) Vorstellungen wie folgt:

„Mental imagery refers to all those quasi-sensory or quasi-perceptual experiences of which we are self consciously aware and which exist for us in the absence of those stimulus conditions that are known to produce their genuine sensory or perceptual counterparts." (Richardson, 1969, S. 2-3, zit. nach Murphy, 1994, S. 486)

Komponenten der motorischen Vorstellungen sind demnach bewusstseinsfähig, was es ermöglicht, sie von der unbewussten und nicht bewusstseinsfähigen motorischen Planung abzugrenzen. Wenn es jedoch um die Vergegenwärtigung der Ziele einer Bewegung geht, ist eine Antizipationsleistung erforderlich. Diese muss aber nicht in notwendiger Weise bewusst sein.

\subsubsection{Vorstellungen und das Gesetz von Fitts}

Das Gesetz von Fitts (Fitts, 1954) besagt, dass die Zeit, die für eine Aufgabe gebraucht wird, logarithmisch mit der Aufgabenschwierigkeit steigt. Genauigkeit geht auf Kosten von Zeit und wenn es um Schnelligkeit geht, geht dies auf Kosten der Genauigkeit, dieses wird als Geschwindigkeits-Genauigkeits-Beziehung bezeichnet. Solodkin et al. (2004) haben Befunde hierzu dargestellt.

Caeyenberghs et al. (2009b) haben das Gesetz von Fitts bei Kindern und Jugendlichen zwischen 6 und 16 Jahren untersucht. Auf die Fragestellung der Erreichbarkeit eines Objektes angewandt, besagt das Gesetz, dass je kleiner und weiter weg das Objekt ist (d.h. je schwieriger es zu erreichen ist), um so länger dauert es, bis das Objekt erreicht wird. Caeyenberghs et al. (ebd.) nutzen die „Virtual Radial Fitts“-Aufgabe. Dabei sollen, von einem Punkt ausgehend, unterschiedlich große Ziele, die in einem Halbkreis angeordnet sind, getroffen werden. Dabei zeigte sich, dass es eine lineare Beziehung zwischen Schwierigkeit und Zeit für die motorische Ausführung gab, und dies gilt schon 
ab einem Alter von 6 Jahren, also für die jüngsten der untersuchten Kinder. Bei der vorgestellten Aufgabe stieg die Korrelation von vorgestellter und motorischer Ausführung mit dem Alter an. Dies wurde mit der, sich mit dem Alter verbessernden Fähigkeit interpretiert, interne mentale Modelle für zukünftige Handlungen zu generieren. Allerdings sagt das Gesetz von Fitts logarithmische Beziehungen voraus, hier wurde eine lineare Beziehung gefunden. 


\subsection{Modelle von Vorstellungen}

Gemäß Farahs Modell der Vorstellungsprozesse (Farah, 1984) und Kosslyns früherem Modell (Kosslyn, 1980) werden Vorstellungen aus dem Langzeitgedächtnis generiert und dann im Zwischenspeicher (visual buffer) bereitgehalten. Dort wird die Aktivierung entdeckt, Vorstellungen können entziffert oder je nach Aufgabe inspiziert werden (siehe Abbildung 1). Farahs Modell entstammt Läsionsstudien, was an sich problematisch ist. Dieses ist zudem noch sehr einfach gehalten. Was sich in der von Kosslyns weiterentwickelten Version noch wieder findet, ist die Beteiligung des Langzeitgedächtnisses und des Zwischenspeichers, während Kosslyn (1994) das Modell hinsichtlich der Verarbeitungsschritte anpasst, die für die Wahrnehmung und Vorstellung gemeinsam sind.

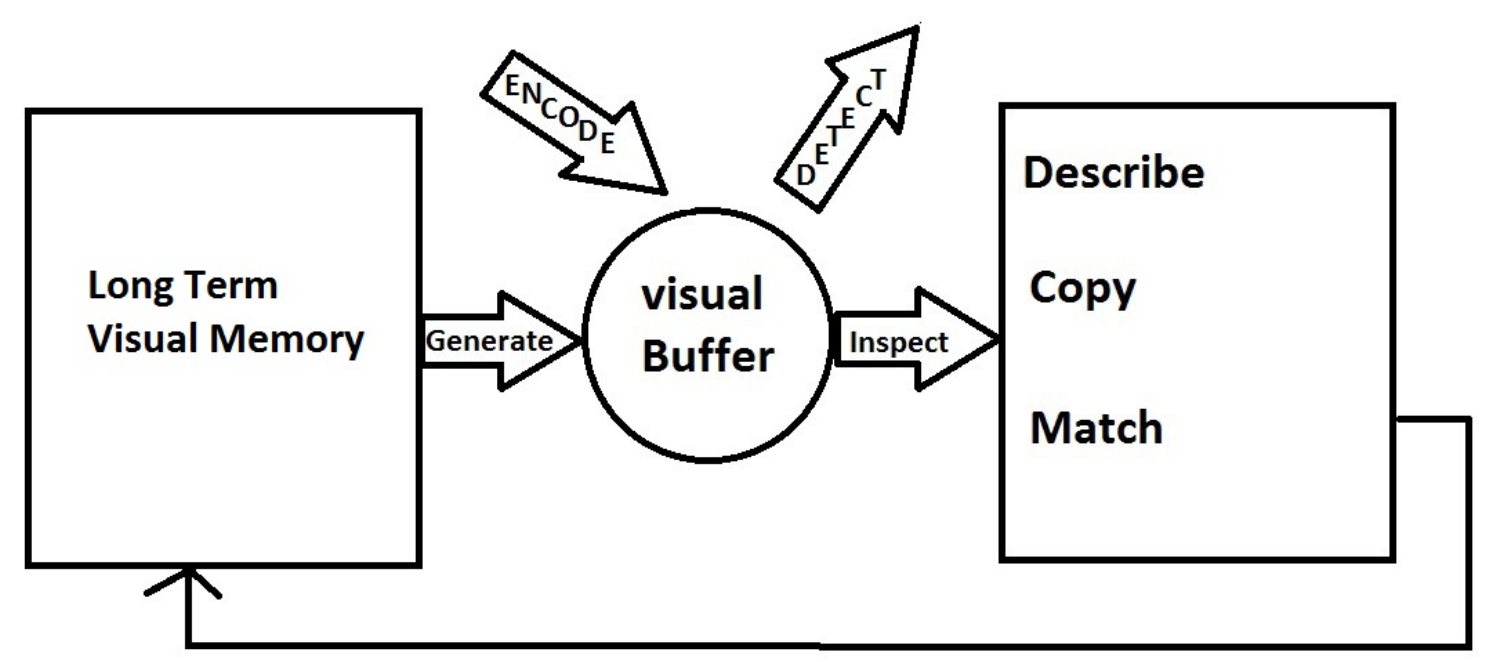

Abbildung 1: Vorstellungsmodell von Farah (1984, S. 250)

\section{Bezug zur eigenen Arbeit}

Farahs Modell ist jedoch für eine erste Aufgabenanalyse hilfreich: Was tun die Kinder, die die unvollständigen Videoaufnahmen sehen? Die hierfür benötigten Prozesse sind womöglich folgende: Aus dem Langzeitgedächtnis werden Bewegungsrepräsentationen in das Arbeitsgedächtnis geholt („generation“). Das Vorstellungsbild kann im Arbeitsgedächtnis zur weiteren Bearbeitung bereitgehalten werden. Demnach kann das Kind die Aufgabe lösen, indem es die vorgestellte Bewegung inspiziert. Doch das Modell gibt keine Hinweise für eine möglicherweise stattfindende mentale Ergänzungsleistung. 
Finke (1980, zit. nach Kebeck, 1997) ging noch davon aus, dass Vorstellungen die frühe visuelle Informationsverarbeitung nicht beeinflussen. Er vergleicht die Wahrnehmung und Vorstellung auf drei zeitlichen geordneten Ebenen der Verarbeitung:

1. Ebene: Wahrnehmung und Vorstellung unterscheiden sich auf unterster Verarbeitungsstufe, d.h. es gibt z.B. keine Nachbilder in der Vorstellung, fehlende Adaptation der Zapfen in der Vorstellung. Zapfen sind Rezeptoren, die zuständig für das Farbsehen sind.

2. Ebene: Nicht immer führen Wahrnehmung und Vorstellung zu gleichen Resultaten. Die Vorstellung gelingt beispielsweise bei abstrakten Worten nur schlecht.

3. Ebene: Auf dieser Verarbeitungsebene sind die Wahrnehmung und die Vorstellung funktional äquivalent, d.h. es werden durch beide Prozesse die gleichen Ergebnisse erzielt.

In der Abbildung 2 wird das Modell von Finke (1986, zit. nach Kebeck, 1997) dargestellt.

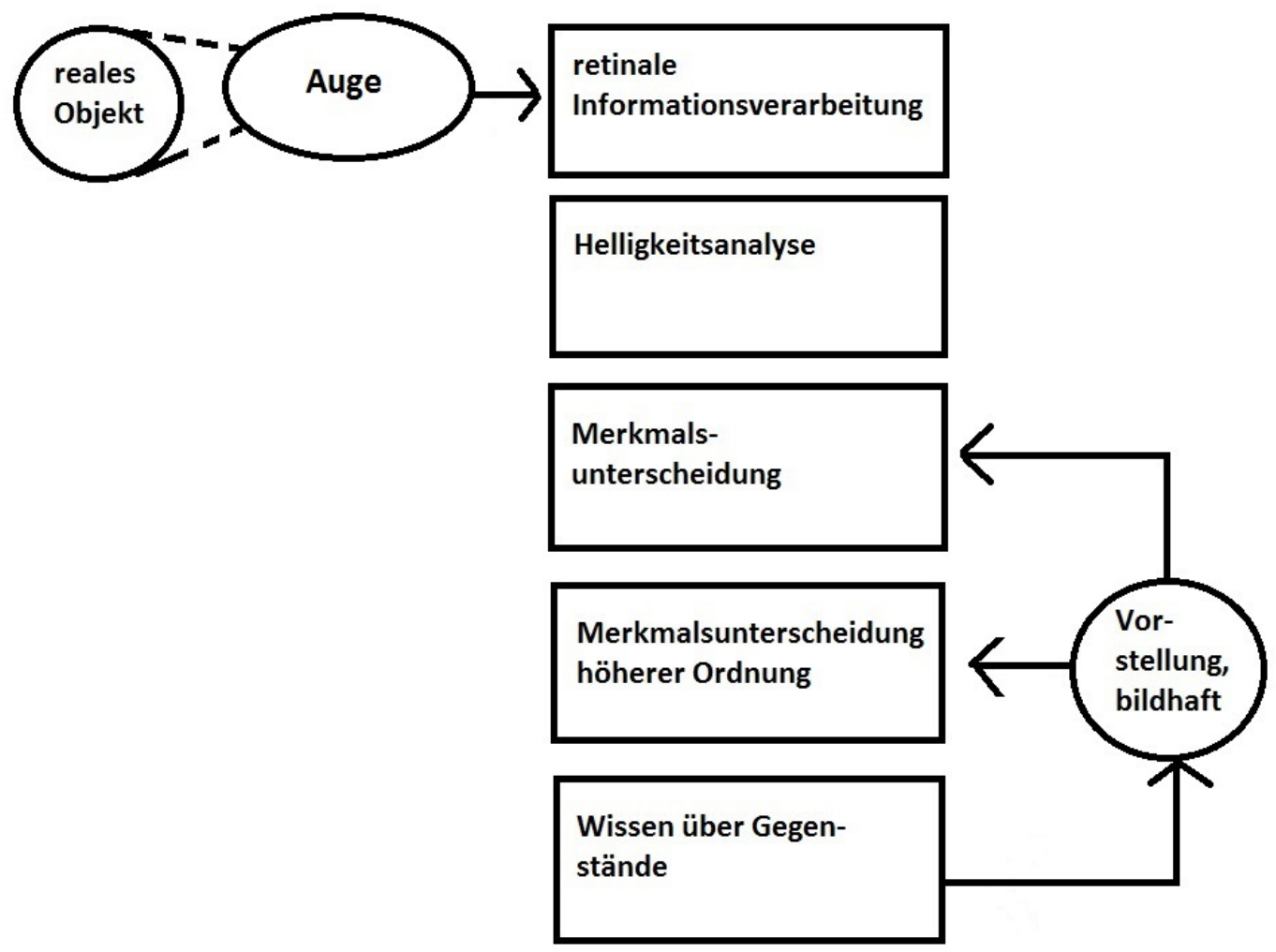

Abbildung 2: Modell von Finke (1986) zum Einfluss von Vorstellungen auf die Wahrnehmung (zit. nach Kebeck, 1997, S. 197) 
Bei Finke (ebd.) hängt die funktionale Äquivalenz von Wahrnehmung und Vorstellung von der Ebene $a b$, in der die Verarbeitung gerade stattfindet. Finke kann erstmals in seinem Modell festhalten, wie die Vorstellungen Einfluss auf höhere Wahrnehmungsprozesse nehmen (siehe Ishai \& Sagi, 1997a, dargestellt im Kap. 6.2.2). Jedoch beinhaltet dieses Modell noch keine Unterscheidung zwischen dem ventralen und dorsalen Pfad der Informationsverarbeitung bei der visuellen Wahrnehmung, wie sie heute bekannt sind (siehe Modell von Goodale \& Milner in Kap. 4.2).

Spezifischer geht Kosslyn (1994) (siehe Abb. 3) von einem Zwischenspeicher (dem visuellen Buffer) aus, der bildliche Informationen während des Vorstellungsprozesses, welcher „top-down“ abläuft, verfügbar macht. Auch die Wahrnehmung greift auf diesen Zwischenspeicher zu, während er den Wahrnehmungsgegenstand enkodiert („bottomup“-Prozess). Im visuellen Buffer gibt es nach Kosslyn (ebd.) ein „Aufmerksamkeitsfenster“, um den interessierenden Bereich zu fokussieren. Wie bei der Wahrnehmung wird für die Vorstellung angenommen, dass gleiche Teilsysteme für die Verarbeitung von Informationen verantwortlich sind. So zum Beispiel das ventrale "WasSystem“ und das dorsale „Wo-System“, welches auch schon in Kap. 4.2 erläutert wurde. Anschließend werden verschiedene Systeme, beispielsweise das assoziative Gedächtnis aktiviert und die Informationen dort weiterverarbeitet.

Im Vergleich zu Finkes Modell (Finke, 1980, 1986) kann die Vorstellung erst geprüft werden, wenn sie in frühe Verarbeitungsebenen gelangt. Dies geschieht im Zwischenspeicher, der im Bereich der primären und sekundären Sehrinde angesiedelt wird (Kosslyn, 1994). 


\section{sonstige \\ Verarbeitungseinheiten}

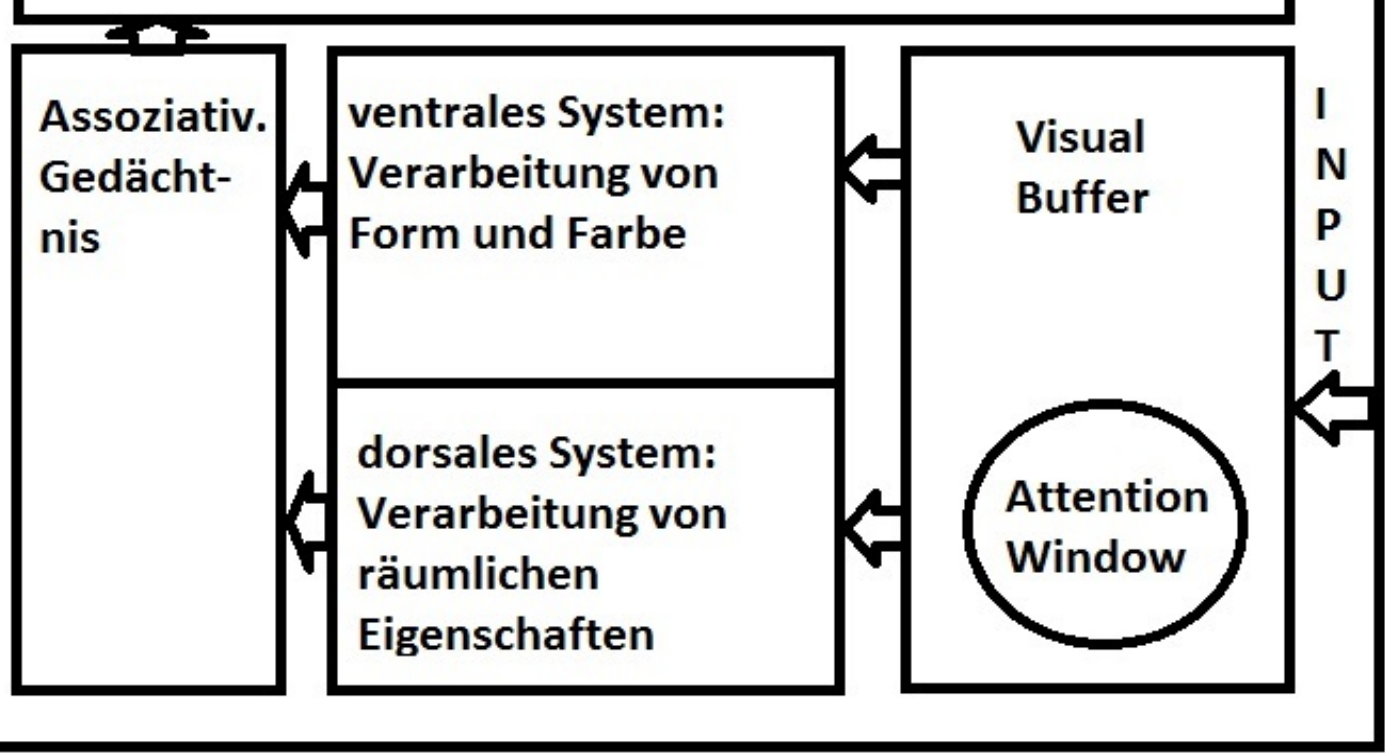

Abbildung 3: Das Prototypen-Modell der Wahrnehmung ähnelt dem des Vorstellungsprozesses, Kosslyn (1994; S. 69 und 383) (vereinfacht und zusammengefasst)

Die Ähnlichkeit des Vorstellungsprozesses mit dem der Wahrnehmung ist bei Kosslyns Modell am ausgeprägtesten. Hier gibt es Hinweise auf mögliche strukturelle und funktionale Äquivalenzen beider Prozesse.

Wie schon im Kap. 3 erwähnt, wird angenommen, dass die Spiegelneuronen die neuronale Grundlage für die mentale Simulation und somit auch die mentale Ergänzung, wie sie hier postuliert wird, bilden. Noch fehlen Ansatzpunkte, diese These in die Modelle zu integrieren. Hier ist weitere theoretische Arbeit notwendig. 


\section{Vergleich von Wahrnehmung und Vorstellung}

Viele Studien haben sich in den letzten Jahren damit beschäftigt, die Prozesse Wahrnehmung und Vorstellung bzw. Handlungsausführung und Vorstellung miteinander zu vergleichen (z.B. Kosslyn et al., 2006). Es gibt nach Wissenslage der Autorin dieser Dissertation bis dato aber nur wenige Arbeiten außerhalb der Neurowissenschaften, die versuchen, Wahrnehmungs- und Vorstellungsprozesse in ihren Unterschieden zu quantifizieren.

Der Vergleich dieser Prozesse in der neurowissenschaftlichen Literatur basiert vielfach auf fMRT-Studien, bei denen die Differenz beider Prozesse diejenigen Gehirnareale angibt, die nur bei einem Prozess aktiv sind (siehe auch Kosslyn (1994) für Kritik).

Einen anderen Ansatz verfolgen beispielsweise Ishai und Sagi (1997a) (siehe Kap. 6.2.2). Zunächst sollen die Gemeinsamkeiten von Wahrnehmung und Vorstellung zusammengetragen und aufgezeigt werden.

\subsection{Gemeinsamkeiten von Wahrnehmung und Vorstellung}

McGinn (2007) schreibt: „Es gibt mithin eine klare Ähnlichkeit zwischen beiden Arten des Erlebens bzw. Erfahrens [gemeint sind Wahrnehmungen und Vorstellungen] - zwischen ihrer Phänomenologie, ihrer Intentionalität und ihrer sinnesbezogenen Modalität ebenso wie einen klaren Unterschied" (McGinn, 2007, S. 15). So sind Wahrnehmungen und Vorstellungen für McGinn (ebd.) beides visuelle Erlebensformen, wobei das Sehen eine Objektpräsenz erfordere, das Sich-Vorstellen nicht. Den Eiffelturm zu sehen, bedeutet etwa seine Form und Farbe sowie den Hintergrund zu sehen, inn vorzustellen bedeutet ebenfalls seine Form, Farbe und den Hintergrund zu sehen. In beiden Fällen ist der gleiche Gegenstand das "intentionale Objekt" des Erlebens (McGinn, 2007, S. 15). Die von McGinn (ebd.) erwähnten Unterschiede werden im Kap. 6.2 behandelt.

Bosshardt (1973) bemerkt, dass die „erlebnismäßige Vergleichbarkeit von Vorstellungen und Wahrnehmung [...] zu der Annahme geführt [hat], daß das Zustandekommen beider Erlebnisarten ähnliche Ursache[n] habe" (Bosshard, 1973, S. 4). Und weiter heißt es: „Die Ähnlichkeit zwischen Vorstellungs- und Wahrnehmungserlebnis besteht in den sinnlichen, sensorischen Erlebniskennzeichen“ (Bosshardt, 1973, S. 6). 


\subsubsection{These der Wahrnehmungsähnlichkeit}

Rollins (2006) nennt die Auffassung, dass mentale Bilder wie Wahrnehmungsbilder sind, die These von der Wahrnehmungsähnlichkeit mentaler Vorstellungen. Nicht immer ist es leicht, Gemeinsamkeiten und Unterschiede von Wahrnehmung und Vorstellung zu trennen. Meist werden vorhandene Unterschiede so interpretiert, dass sie in die Gesamtthese zur Wahrnehmungsähnlichkeit passen. Kosslyn, Ganis und Thompson (2010) schreiben dazu: "Mental imagery is like perceiving, but in the absence of an immediate appropriate sensory stimulation" (Kosslyn, Ganis und Thompson, 2010, S. 3). So heißt es weiter:

„Rather, mental images consist of internal representations of the same type as those that arise during the early phases of like-modality perception. Mental images are internal representations that are based on information stored in memory. In contrast, perception occurs when information is registered directly from the senses." (Kosslyn, Ganis und Thompson, 2010, S. 3)

Wahrnehmungsähnlichkeit bedeutet nicht, dass die Prozesse identisch sind, sondern dass sich Wahrnehmung und Vorstellung viele Prozessmechanismen teilen. Außerdem wird der Anteil derjenigen Gehirnareale, die nur bei der Vorstellung aktiv sind, auf ca. zwei Drittel der Areale geschätzt, die bei der Wahrnehmung aktiv sind (Kosslyn, 2005). Ob der primäre visuelle Kortex (Areal V1) bei der Vorstellung aktiv ist, hängt nach Kosslyn und Thompson (2003, zit. nach Eysenck, 2012) von folgenden drei Faktoren ab:

1. die Art der Aufgabe: Es sollten in der Vorstellung Details erfasst werden, damit das Areal V1 aktiv ist.

2. die Art des bildgebenden Verfahrens: Bei fMRT-Techniken war dies am häufigsten der Fall.

3. Formerfassungsaufgaben statt Scanning-Aufgaben: Bei Vorstellungsaufgaben, in denen die Form im Vordergrund steht, statt sich das Objekt in Bewegung vorzustellen.

Übrigens gibt es auch bei Aufgaben zur Mentalen Rotation keine Aktivierung früher visueller Areale (Mast, 2005).

Ganis, Thompson und Kosslyn (2004) weisen ebenfalls darauf hin, dass es bei Vorstellungs- und Wahrnehmungsaktivitäten Areale gibt, die bei der Wahrnehmung aktiv sind, auch in der Vorstellung aktiv sind.

Bei der Wahrnehmung wird nach Norman (1979, zit. nach Kebeck, 1997) die ankommende sensorische Information interpretiert und bei der Vorstellung wird sich an 
diese Interpretation erinnert. Dieser Auffassung nach werden Vorstellungen mit Erinnerungsbildern gleichgesetzt, während die antizipatorischen Vorstellungen und die Möglichkeit, in der Vorstellung neue Kombinationen von Bewegungen herzustellen, hier vernachlässigt werden.

\subsubsection{Zeitliche Ähnlichkeit und biomechanische Gesetze}

Mit dem Forschungsparadigma der Mentalen Chronometrie werden Ähnlichkeiten in der Vorstellung von Bewegungen und der Bewegungsausführung dadurch aufgezeigt, indem die Zeitdauer der mentalen Vorstellung der Handlung mit der realen Handlung verglichen wird.

Guillot und Collet (2005) betonen die zeitlichen Merkmale der motorischen Vorstellungen, die sie mit realen Handlungen und Bewegungen gemein haben. Diese seien durch Merkmale der physischen Bewegung und deren biomechanischen Einschränkungen beeinflusst. „Specifically, the time required to select a grip increases as a function of the angular distance to the location of the selected posture along the shortest biomechanically plausible trajectory“ (Guillot \& Collet, 2005, S. 11). Gemeinhin gelten große Ähnlichkeiten zwischen vorgestellten und realen Bewegungen, die aber je nach Art der Bewegung, Aufgabenschwierigkeit bzw. Schwierigkeit der Bewegung überoder unterschätzt werden. Beispielsweise benötigen komplexe Aufgaben mit hohen Aufmerksamkeitsanforderungen in der Vorstellung einen längeren Zeitraum.

Auch Instruktionen können die Dauer der vorgestellten Bewegung beeinflussen. Unterschätzt werden Bewegungsvorstellungen in ihrer Dauer, wenn Athleten vor dem Wettkampf Mentales Training betreiben oder sich nur die dynamischen Phasen der Bewegung vorstellen. Bei sehr guten Athleten (Experten) entspricht jedoch die vorgestellte Zeit auch der tatsächlichen Zeit. Auch die Zeit, in der das Mentale Training durchgeführt wird, beeinflusst die Dauer der Vorstellung. Hier wurde der Versuch während des Trainings mit dem Ergebnis durchgeführt, dass die Dauer der Vorstellung länger wurde. Wenn genug Zeit vorhanden ist, so stellen sich demnach die Sportler eher genauere Bilder vor und die Vorstellung dauert länger als die aktuelle Bewegung (Guillot \& Collet, 2005).

Bei mittlerer Expertise dauern Vorstellungen länger als die tatsächlich ausgeführte Bewegung, im Vergleich zu Experten höheren Niveaus und Novizen (Reed, 2002).

Ein Hauptexperiment führten Decety, Jeannerod und Prablanc (1989) durch. Dabei sollten Probanden blind zu Zielen gehen, die in unterschiedlich bekannter Entfernung standen. Bei Erwachsenen, die in der Nutzung mentaler Vorstellungen instruiert wurden, 
waren die Zeiten zwischen vorgestelltem und ausgeführtem Gehen gleich. Sie machten keine Fehler hinsichtlich der Richtung des Gehens, überschätzen aber leicht die Länge des Weges. Gleichzeitig wiesen die Probanden der Kontrollgruppe, welche keine Instruktionen erhielten, ihre Vorstellungen zu nutzen, größere Fehlerwerte auf. Folgende Ergebnisse weisen darauf hin, dass es auch Unterschiede bei Handlungsausführung und Vorstellung gibt: In einem zweiten Experiment von Decety et al. (ebd.) sollten die Versuchspersonen zusätzlich ein Gewicht auf den Schultern tragen. Es zeigte sich, dass die Dauer bei der Vorstellungsbedingung 30\% länger war als in der unveränderten Bewegungsbedingung. Diese Ergebnisse interpretierten die Autoren im Hinblick auf neuronale Parameter im motorischen Programm, die für Handlungen, egal ob sie tatsächlich ausgeführt oder mental vorgestellt werden, eine Rolle spielen. Kritisch ist anzumerken, dass vorhandene Unterschiede zumeist im Sinne der Ähnlichkeitsthese interpretiert werden.

Auch Sirigu et al. (1995, 1996, zit. nach Crammond, 1997) führten ein Experiment mit dem Paradigma der Mentalen Chronometrie durch. Versuchspersonen sollten Fingerbewegungen nach einem Metronomschlag vollziehen, wobei sich der Daumen und die Fingerkuppen der anderen Finger abwechselnd treffen sollten. Dabei wurde das Metronom immer schneller, bis die Probanden die Bewegung nicht mehr ausführen bzw. sich vorstellen konnten. Sie verglichen die Zeitdauer für aktiv ausgeführte und vorgestellte Bewegungen. Diese waren sehr ähnlich, so dass die schnellste Frequenz beim Ausführen auch der schnellsten bei der Vorstellung entsprach. Auch für das wiederholte Zeigen von Handgesten und andere Zeigebewegungen sowie deren Vorstellungen brauchten die Probanden in etwa die gleiche Zeit. Diese wurde größer, je kleiner das Ziel der Bewegung war. Diese Geschwindigkeits-Genauigkeits-Beziehung wird auch als das Gesetz von Fitts bezeichnet (siehe Kap. 5.4.3).

Es zeigten Papaxanthis, Pozzo, Kasprinski und Berthoz (2003, zit. nach Moran, 2004), dass bei Astronauten sowohl vor als auch nach einem sechsmonatigen Flug ins All die vorgestellte und tatsächlich ausgeführte Zeit bei einfachen Alltagsbewegungen (Treppe steigen, Hüpfen, Laufen) ähnlich waren. Interpretiert wurde dies mit ähnlichen Prozessen bzw. einer ähnlichen neuronalen Struktur als Grundlage für die Adaptationsprozesse.

\subsubsection{Funktionale Äquivalenz}

Je ähnlicher die vorgestellten und aktuellen Zeiten bei der Bewegungsausführung sind, 
desto eher kann davon ausgegangen werden, dass Vorstellungsprozesse den Bewegungsabläufen funktional ähnlich sind (Decety, Jeannerod \& Prablanc, 1989, zit. nach Moran, 2004).

Ergänzend sollen hier Studien, die mit anderen Paradigmen durchgeführt wurden, referiert werden, die funktionale Äquivalenzen zwischen der Wahrnehmung, Vorstellung und Bewegungsausführung aufzeigen sollen:

- Patienten mit Schäden am motorischen System haben auch Defizite bei motorischen Vorstellungen: So fand Dominey et al. (1995, zit. nach Currie \& Ravenscroft, 1997) heraus, dass Parkinsonpatienten, welche rechtsseitig betroffen waren, beim Fingertapping-Test mit der rechten Hand sowohl beim Ausführen als auch beim Sich-Vorstellen der Bewegungsaufgabe schlechter abschnitten als beim linksseitigen Test.

- Auch bei Aufgaben mit weniger großem kognitiven Anteil kann das Mentale Training wirken: Es wurde gezeigt, dass nicht nur beim aktiven Ausüben, sondern auch durch Mentales Training Kraftparameter gesteigert werden können (Yue \& Cole, 1992, zit. nach Currie \& Ravenscroft, 1997). Jeannerod (1994) zeigte diesbezüglich, dass während der Vorstellung keine Muskelaktivität mit der Elektromyografie (EMG) zu messen und auch die selektiv gesteigerte Motivation keine erklärende Variable war. Dies ist als eine Erweiterung bisheriger Kenntnisse zum mentalen Training anzusehen.

Überlappende, aber nicht gleiche neuronale Strukturen werden wie folgt im Sinne der These der funktionalen Äquivalenz interpretiert:

- Die neuronalen Strukturen, die beim Bewegen und Sich-Vorstellen von Bewegungen aktiv sind, überlappen sich zum Teil. So sind der Prämotorische Kortex, die Basalganglien und das Kleinhirn während beider Prozesse aktiv (Decety et al., 1990; Fox et al., 1987; Roland et al., 1980, zit. nach Currie \& Ravenscroft, 1997), während der Primärmotorische Kortex bei Vorstellungsvorgängen nicht aktiv ist (Roland et al., 1980). Dies kann mit einem neuronalen "Tor“ erklärt werden, das sich analog zu einem Hemmmechanismus schließt, wenn sich etwas vorgestellt wird (Currie \& Ravenscroft, 1997).

- Ingvar und Philipsson (1977, zit. nach Jeannerod, 1994) zeigten mittels Studien über den cerebralen Blutfluss eine Aktivität des Frontallappens bei Vorstellungen 
von Bewegungen und ein aktives Roland-Areal bei ausgeführten Bewegungen. Das supplementärmotorische Areal war bei beiden Prozessen aktiviert (Fox et al., 1987, zit. nach Jeannerod, 1994).

Hinweise für funktionale Ähnlichkeiten gibt es auch bei Studien, die physiologische Vorgänge bei vorgestellter und tatsächlich ausgeführter Bewegung untersuchen. Dabei trennen Engelkamp und Zimmer (2006) drei Bereiche: die Muskelaktivität, Reaktionen des autonomen Nervensystems und die Gehirnaktivitäten. Schon Jacobson (1930, zit. nach Engelkamp \& Zimmer, 2006) erkannte, dass bei mentalen Vorstellungen von Bewegungen die Seite des Körpers Muskelaktivität zeigt, die sich vorgestellt wurde, nicht aber die kontralaterale Seite. Zu den Parametern des autonomen Nervensystems gehören Herzschlag, Atemfrequenz und Puls. Bei der Vorstellung werden sofort Änderungen dieser Parameter beobachtet. Engelkamp und Zimmer (2006) weisen darauf hin, dass dies auf die Vorbereitung der motorischen Reaktion, nicht aber auf die Veränderung der Stoffwechselprozesse infolge der Bewegung zurückgeht. Schließlich kann von einer Aktivität im supplementär-motorischen Areal (SMA) ausgegangen werden, wenn sich Menschen Bewegungen vorstellen und sie ausführen. Wenig Hinweise gibt es laut den Autoren auf die Beteiligung des Primärmotorischen Kortex bei der Vorstellung. Er ist scheinbar nur bei der Bewegung selbst aktiv.

\subsubsection{Topografische Organisation von Wahrnehmung und Vorstellung}

Jeannerod (1994) resümiert die Auffassungen zu visuellen Vorstellungen wie folgt: Weil die ankommende Information nebeneinanderliegender Rezeptoren in der Retina im Gehirn auch durch nebeneinanderliegende Gehirnstrukturen abgebildet werden (sogenannte topografische Organisation), beinhaltet die Mentale Repräsentation die metrisch-räumlichen Eigenschaften der repräsentierten Szenen und Objekte. Diese Annahmen werden seiner Meinung nach durch folgende Befunde gestützt:

1) Von Geburt an blinde Menschen behalten die räumliche Organisation in ihrer Vorstellung (Kerr, 1983, zit. nach Jeannerod, 1994). Die räumliche Vorstellung muss sich, so Jeannerod (1994), nicht notwendigerweise auf die visuelle Erfahrung stützen. Erklärt werden kann der Befund durch die räumlichen Eigenschaften der Gehirnregionen, die in das Generieren von Vorstellungsbildern involviert sind.

2) Ein anderes Argument bezieht sich auf einen Patienten, bei dem der Okzipitallappen entfernt wurde. Bei inm war die Größe des mentalen visuellen 
Feldes gleicherweise eingeschränkt wie das tatsächliche visuelle Feld (Farah et al., 1992, zit. nach Jeannerod, 1994).

3) Auch Patienten mit Hemineglect, welche die visuellen Reize einer Raumseite ignorierten, taten dies auch in der Vorstellung (Bisiach und Luzzatti, 1978, zit. nach Jeannerod, 1994).

4) Schließlich stimmt das Muster der Gehirnaktivität bei Vorstellungs- und Wahrnehmungsprozessen im Großen und Ganzen überein (Goldenberg et al., 1989 a,b; Roland \& Friberg, 1985, zit. nach Jeannerod, 1994).

Einen weiteren Hinweis für die Ähnlichkeit von Wahrnehmung und Vorstellung fasst Mast (2005) zusammen: Sowohl der Aufbau des visuellen Kortex als auch dessen Aktivierung bei Vorstellungsaufgaben erfolge retinotrop. Das bedeutet, dass die räumlichen Eigenschaften des Reizes auch räumlich abgebildet werden. Für Vorstellungen wurde gezeigt, dass der primäre visuelle Kortex aktiv ist und die Aktivierung des Areal 17 von der Größe des jeweiligen Vorstellungsbildes abhängt. Das Areal 17 entspricht dabei der primären Sehrinde. Bei kleinen Bildern in der Vorstellung liegt das Aktivierungsmaxima im primären visuellen Kortex posterior und bei größeren Bildern anterior, wie es auch bei der Wahrnehmung zu erwarten ist (Kosslyn, Thompson, Kim \& Alpert, 1995, zit. nach Mast, 2005).

Diese Befunde sind für die schon erwähnte „Imagery Debatte“ von Bedeutung, nach der zwei Parteien darüber uneinig sind, ob es zusätzlich zu propositionalen Repräsentationen auch bildhafte gibt (siehe Pylyshyn (1981) für erstere Ansicht und Kosslyn (1980, 1994) für letztere Auffassung). 


\subsection{Unterschiede von Wahrnehmung und Vorstellung}

\subsubsection{Qualitative Unterschiede}

Dieser Abschnitt beinhaltet Aspekte, die sich auf Unterschiede zwischen Wahrnehmung und Vorstellung beziehen:

1. Stern (1935, zit. nach Bosshardt, 1973, S. 3) spricht vom Kriterium der „Anschaulichkeit“: Wahrnehmungen seien eher konkret, das Denken abstrakt und Vorstellungen befinden sich in der Mitte des Kontinuums. Mittels eines zweipoligen Kriteriums trennt Jaspers Wahrnehmungen und Vorstellungen. Wahrnehmungen sind nach Jaspers (1965, S. 59f, zit. nach Bosshardt, 1973, S. 3) durch ihre „Leibhaftigkeit“ und Vorstellungen durch ihre „Bildhaftigkeit“ gekennzeichnet. So erscheinen bei ihm Wahrnehmungen im „äußeren, objektiven“ Raum und Vorstellungen im „inneren subjektiven“ Raum.

2. Vorstellungen sind per Definition ohne Sinnesinformationen erzeugte mentale Bilder. Die Unterschiede von Vorstellung und Wahrnehmung werden wie folgt definiert: „... imagery, unlike perception, does not require low-level organizational processing; and perception unlike imagery, does not require us to activate information in memory when the stimulus is not present ..." (Kosslyn, Ganis \& Thompson, 2010, S. 5). Während die Wahrnehmung das Sehen eines Reizes beinhaltet, ist die mentale Vorstellung eine Erfahrung des Sehens ohne sensorischen Input. Das Gedächtnis bildet somit die Grundlage dieser Erfahrung. Auf der Wahrnehmungsseite geht es um die Identifikation, das Wiedererkennen von Reizen und die Figur-Grund-Erkennung. Die Wahrnehmung kann sowohl von Gedächtnisinhalten (also Glaubenssätzen, Wissen, Erwartungen und Zielen) beeinflusst werden (sogenannter „top-down“-Prozess) als auch vom Stimulus selbst („bottom-up“) (Kosslyn, 2005, Kosslyn \& Thompson, 2000). Mehr noch wird hier der Unterschied zwischen Wahrnehmung und Vorstellung deutlich: "Mental images are internal representations that are based on information stored in memory. In contrast, perception occurs when information is registered directly from the senses" (Kosslyn, Ganis \& Thompson, 2010, S. 3).

Die oben genannte Definition impliziert, dass bei Vorstellungen „top-down“-Prozesse vorliegen, da die Reize für die „bottom-up“-Analyse fehlen. Damit kann die Hypothese, dass Experten ihr Wissen einsetzen, um Vorstellungen zu generieren, begründet 
werden.

Kann es möglich sein, dass Vorstellungen auch über „bottom-up“-Prozesse geniert werden, wie es in den Vorüberlegungen angeregt wurde? Gelte diese Prämisse, würde die Wahrnehmung die Vorstellung beeinflussen. Der Einfluss von Vorstellungen auf die Wahrnehmung wird bei Ishai und Sagi (1997a,b) gezeigt (siehe Kap. 6.2.2 und 7.2).

3. Vorstellungen bestehen im Gegensatz zu Wahrnehmungen aus vereinfachten strukturellen Beschreibungen von Objekten (Eysenck, 2012). Eysenck ergänzt: „In general, the information available in visual perception is more detailed and can be used more flexibly than that available in visual images" (Eysenck, 2012, S. 58).

Dies gilt für neurophysiologische und neuroanatomische Studien, welche Unterschiede zwischen Vorstellungen und Wahrnehmungen aufzeigen, siehe Eysenck (2012).

4. McGinn (2007) kritisiert die Auffassung von der Wahrnehmungsähnlichkeit der Vorstellungen. Vorstellungen und Wahrnehmungen unterscheiden sich nach McGinn (ebd.) bezüglich folgender Kriterien:

a) Wille (Vorstellungen unterliegen dem Willen, Wahrnehmungen nicht)

b) Informationsgehalt (Vorstellungen sind nicht informativ)

c) Gesichtsfeld (Vorstellungen sind nicht durch das Gesichtsfeld begrenzt)

d) Sättigung (Vorstellungen sind „lückenhaft, ungenau und unzusammenhängend“ (McGinn, 2007, S. 35))

e) Aufmerksamkeit (bei Wahrnehmungen kann eine Wahl zwischen Aufmerksamkeit und Nichtaufmerksamkeit getroffen werden, nicht so bei Vorstellungen)

f) Abwesenheit von Objekten (bei Vorstellungen sind Objekte abwesend)

g) Wiedererkennen (McGinn (ebd., S. 41) schreibt: „[...] ich kann das Objekt meiner Vorstellung nicht fehlidentifizieren“, und weiter heißt es, dass bei Vorstellungen „kein Akt des Wiedererkennens erforderlich“ ist.)

h) Denken (McGinns These ist, dass nicht gleichzeitig an etwas gedacht und es sich vorgestellt werden kann.)

i) Okklusion (Vorstellungen sind nicht okklusiv. Das bedeutet, dass es einer Person möglich ist, sich gleichzeitig etwas vorzustellen und etwas anderes wahrzunehmen. Wahrnehmungen hingegen sind okklusiv, das heißt Wahrnehmungen stören sich gegenseitig.)

j) Ableitung versus Reduktion (Das Gedächtnis ist „selektiv und kreativ“ (McGinn, ebd., S. 45). Daher sind Erinnerungsbilder der „Output" oder Ableitungen von 
Wahrnehmungen. Vorstellungen sind Reduktionen von Wahrnehmungen, auf denen sie gründen.)

k) Beziehungen zwischen dem Wahrnehmen, dem Sich-Vorstellen und dem Denken: "ICh kann nicht gleichzeitig eine Wahrnehmung und eine Vorstellung haben, deren Inhalt derselbe ist" (McGinn, ebd., S. 48). Und weiter heißt es: "Man kann eindeutig über ein Objekt nachdenken, während man es sieht, aber man kann sich dabei von ihm keine Vorstellung bilden" (ebd., S. 49). "Wahrnehmungen und Gedanken sind unterschiedliche Arten geistiger Gegebenheiten mit unterschiedlichen Arten von Intentionalität“ (ebd., S. 49).

McGinn weist somit den Gedanken zurück, dass Vorstellungen (ganz oder teilweise) Wahrnehmungen sind. „Vorstellungen sind keine Konstrukte aus Wahrnehmungen und Gedanken [...]. Sie bilden eine eigene Kategorie des Geistigen [...]" (McGinn, 2007, S. $50)$.

\subsubsection{Quantitative Unterschiede}

Es gibt aber auch Studien, die quantitative Unterschiede zeigen: Ein Beispiel hierfür stellt die Untersuchung von Orliaguet und Coello (1998) dar. Sie untersuchten Unterschiede zwischen tatsächlichem und vorgestelltem Golfschlag (Paradigma der Mentalen Chronometrie). Hierbei handelt es sich um eine Bewegung mit relativ kurzer Dauer. Während sich bei der Bewegungsausführungsbedingung die Bewegungsamplitude proportional zur Zieldistanz vergrößert und die Bewegungsdauer unabhängig von der Distanz der Bewegung ist, sind die Ergebnisse für vorgestellte Bewegungen sehr verschieden davon: Die vorgestellte Bewegungsdauer ist abhängig von der Bewegungsdistanz und generell länger als die tatsächliche Bewegung.

Der Versuch, Unterschiede zwischen Wahrnehmung und Vorstellung im Rahmen eines speziellen Untersuchungsparadigmas von Ishai und Sagi (1997a,b) zu quantifizieren, wird im Folgenden dargestellt. Es wird darauf auch in der Diskussion der Ergebnisse (siehe Kap. 8.9) zurückgekommen.

Ishai und Sagi (1997a) haben in ihrem Experiment zunächst untersucht, wie Vorstellungen die Wahrnehmung von Linienmustern erleichtern können. Drei Bedingungen wurden wie folgt gestaltet:

a) In der Wahrnehmungsbedingung sollte der mittlere von drei Reizen entdeckt werden. Jeder der drei Reize bestand dabei aus einem Linienmuster mit 
bestimmter Orientierung, wobei die Längskanten der Linien verschwammen.

b) In der Kontrollbedingung waren außen keine Reize vorhanden, in der Mitte waren die oben beschriebenen Linien dargestellt.

c) In der Vorstellungsbedingung sollten sich die äußeren Reize vorgestellt werden, in der Mitte waren wieder dieselben Linien vorhanden.

Die Orientierung der Linienmuster variierte von Versuch zu Versuch ebenfalls. Es wurden jeweils die Wahrnehmungsbedingung alternierend mit der Kontrollbedingung bzw. die Wahrnehmungsbedingung alternierend mit der Vorstellungsbedingung gezeigt.

Als Ergebnis wurde sowohl bei der Wahrnehmungsbedingung als auch der Vorstellungsbedingung eine Reduktion der Wahrnehmungsschwelle gefunden, was eine erleichterte Wahrnehmung bedeutet. Es konnte quantifiziert werden, in wieweit sich dabei die Vorstellung von der Wahrnehmung unterscheidet. Die Reduktion der Schwelle war in der Vorstellungsbedingung $50 \%$ weniger stark als in der Wahrnehmungsbedingung. In der Kontrollbedingung waren die Schwellen im Vergleich zur Basisbedingung leicht erhöht (siehe Abb. 4).

Dieser Effekt war abhängig von der Orientierung der Linien und abhängig davon, vor welchem Auge sie präsentiert wurden. Bei einer Verzögerung zwischen der Wahrnehmungsbedingung und der Vorstellungsbedingung von über 5 Minuten wurde kein Induktionseffekt festgestellt, das heißt, die Wahrnehmung wurde nicht erleichtert. Es wurde von den Autoren angenommen, dass für die Aktivierung eines Gedächtnissystems, das Vorstellungen aktiviert, Bewusstsein benötigt wird (Ishai und Sagi, 1998).

Allerdings wurden sehr kleine Versuchspersonenzahlen verwendet. Besonders hervorhebenswert ist diese Studie von Ishai und Sagi (1997a) dennoch, weil durch sie die Quantifizierung von Einflüssen der Vorstellung auf die Wahrnehmung ermöglicht wird (siehe Abb. 4). Dabei ist die abhängige Variable von der in dieser Dissertation gewählten verschieden. Während bei Ishai und Sagi (1997a) die Wahrnehmungsschwelle gemessen wurde, steht hier in dieser Dissertation die Fehlerrate im Vordergrund. In der vorliegenden Arbeit können somit folgende Leistungen quantifiziert werden: In welchem Ausmaß weichen Vorstellungsleistungen von Wahrnehmungsleistungen ab, wenn bei der Vorstellungsbedingung Teile der Bewegungssequenzen abgedeckt werden? Gibt es in der Vorstellung eine mentale Ergänzung? Vergleiche hierzu Kap. 8.3.2. 


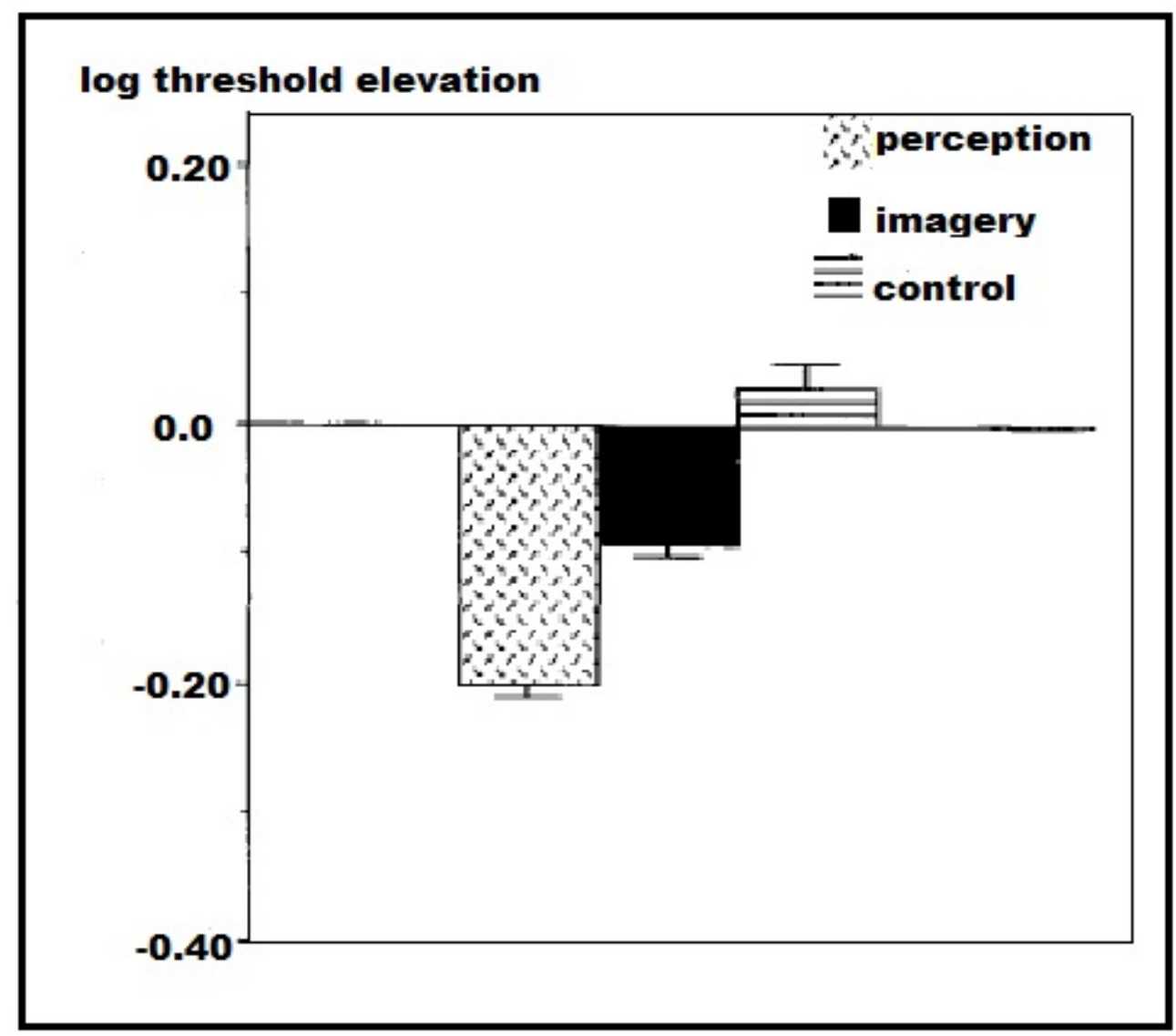

Abbildung 4: Ergebnisse des Experiments urspr. nach Ishai und Sagi (1997a, S. 485); die vorstellungsinduzierte Erleichterung der Wahrnehmungsschwelle 


\section{Stand der Forschung}

\subsection{Motorik, Wahrnehmung und Vorstellung}

Die folgenden Untersuchungen zur motorischen Fertigkeiten, Wahrnehmung und Vorstellung und deren gegenseitige Beeinflussung stellen einen Überblick zum Forschungsstand dar.

\subsubsection{Motorische Fertigkeiten als abhängige Variable}

Das Beispiel Mentales Training zeigt, welchen Einfluss das Training von Bewegungsvorstellungen auf das Lernen von Bewegungen hat. Eine Reihe von Studien (darunter auch Metaanalysen) zeigen die Wirkung von Mentalem Training auf Lernprozesse: „Mentally performing a motor task generally has a positive effect on learning“ (Driskell et al., 1994; Feltz \& Landers, 1983; Grouios 1992; Hinshaw, 1991; Sheikh \& Korn, 1994, zit. nach Féry, 2003, S. 1).

In einer Metaanalyse, welche die Effektivität mentalen Trainings über 60 Studien hinweg bestimmte, wurde von Feltz und Landers (1983, zit. nach Murphy, 1994) eine Effektstärke von 0.48 für das Mentale Training gefunden. Der Einschluss weiterer 14 Studien in einer Folgestudie (Feltz et al., 1988, zit. nach Murphy, 1994) korrigierte diesen Wert auf 0.43. Der Effekt war größer für Aufgaben mit eher kognitiven als motorischen Anteilen (Murphy, 1994). Als kritisch wird bei Metaanalysen stets angeführt, dass die Studien zum Teil nicht vergleichbar seien. Oder es kann auch der Anteil der Studien, die wegen methodischer Mängel nicht in die Metaanalyse eingeschlossen werden, teilweise sehr hoch sein (Bortz \& Döring, 2006). Zudem werden viele Studien, deren Ergebnisse negativ im Sinne der Hypothese sind, nicht publiziert und können somit keinen Eingang in die Metaanalyse finden.

Von Atienza, Balaguer und Barcía-Merita (1998) wurden 9- bis 12-jährige Tennisspieler zufällig in folgende drei Gruppen geteilt und hinsichtlich des Aufschlags über 24 Wochen verglichen: A) eine praktisch übende Gruppe, B) eine Gruppe mit praktischen Übungen kombiniert mit Videomodellen und C) eine Gruppe, die zusätzlich zu Aufgaben der Gruppe B auch noch ein mentales Vorstellungstraining durchführte. Als Ergebnis fanden sie eine gleich gute Leistung in den letzten beiden Gruppen, welche zugleich besser als die Leistung der ersten Gruppe war. Es wurde hier also kein zusätzlicher Nutzen von Mentalem Training gegenüber dem Beobachten von Videomodellen gefunden. Dies 
spricht für die These der Ähnlichkeit von Wahrnehmung und Vorstellungen.

Dass verschiedene Arten von Vorstellungen den Erwerb motorischer Fertigkeiten jeweils unterschiedlich beeinflussen, haben Kim, Singer und Tennant (1998) durch den Vergleich von auditorischen, visuellen und kinästhetischen Vorstellungen herausgefunden. 60 Erwachsene wurden auf fünf Gruppen aufgeteilt, darunter die oben genannten Vorstellungsarten und zwei Kontrollgruppen, wobei eine davon irrelevante Tätigkeiten ausführten, die anderen nicht beschäftigt waren. Es wurde gezeigt, dass nur die auditorischen und die kinästhetischen Vorstellungen zu einer besseren Genauigkeit bei der Ausführung eines Schlags führten.

\subsubsection{Wahrnehmung als abhängige Variable}

Calvo-Merino et al. (2005) fanden eine erhöhte Aktivität des Gehirns bei Tänzern, welche Tanzsequenzen sahen, mit denen sie sowohl motorische als auch visuelle Erfahrung hatten. Calvo-Merino et al. (2006) kontrollierten die Konfundierung von motorischer und visueller Erfahrung. Dabei zeigte sich, dass gleichgeschlechtliche Bewegungen besser erkannt wurden als andersgeschlechtliche. Bei letzteren Bewegungen bestehen zwar visuelle, nicht aber motorische Erfahrungen. Das bedeutet, motorische Erfahrung hat für die Wahrnehmung eine größere Bedeutung als die visuelle Erfahrung.

Die motorische Erfahrung hat auch Einfluss auf das Wiedererkennen von Tischtennisschlägen (Bischoff \& Kollegen, 2012). Bei diesem Experiment wurden sogenannte „point-light-Figuren“ präsentiert, bei denen an Schulter, Ellenbogen und dem Handgelenk eines gegenüberstehenden Tischtennisspielers befestigte reflektierende Marker oder auch Lichtpunkte gezeigt werden, welche als sich bewegende Figuren erkannt wurden. Wurden den Probanden eigene Bewegungssequenzen untergemischt, dann konnten diese bedeutsam besser erkannt werden als Bewegungen anderer oder rotierte Bewegungen. Die Untersuchung mit dem fMRT ergab bei der Beobachtung eigener Bewegungen eine stärkere Aktivierung in Teilen des Parietallappens und in Teilen des Frontalkortex, welche anders ausfiel als bei der Beobachtung sich bewegender anderer Personen. Mit der „point-light“-Methode kann die dynamischen Informationen der Bewegungen dargestellt werden. Auch auf Körperformen kann indirekt geschlossen werden. Diese Methode entwickelte Johansson (1973) nach Forschungen von Marey (1895/1972).

Hohmann und Kollegen (2011) haben den Einfluss unterschiedlicher motorischer und visueller Erfahrung auf das Erkennen von a) einer Handlung und b) der Person, die diese 
Handlung ausführt, untersucht. Dabei sollten Experten im Basketballspiel und Novizen in dieser Sportart dribbelnde Spielhandlungen oder Spieler aufgrund von „point-lightFiguren“ identifizieren. Dabei zeigte sich in a) kein Einfluss motorischer und visueller Erfahrung, d.h. es wurden Handlungen nicht besser erkannt, wenn sie von einem selbst oder von Teamkollegen ausgeführt wurden. In b) zeigte sich ein Einfluss motorischer Erfahrung, denn sich selbst zu erkennen war leichter als den Teamkollegen zu erkennen.

Der Einfluss, welcher eine gerade ausgeführte Handlung auf die Wahrnehmung von Handlungen hat, wurde von Hamilton, Wolpert und Frith (2004) untersucht. Dabei sollten die Probanden das Gewicht einer Kiste schätzen, die von einer anderen Person gehoben wurde, während die Probanden ebenfalls diese Kiste hoben. Es zeigte sich, dass eine Kiste leichter eingestuft wurde, wenn die Probanden selbst die schwere Kiste hoben und umgekehrt wurde eine Kiste schwerer eingestuft, wenn sie selbst eine leichte Kiste anhoben.

Bei einem motorischen, nicht aber visuellem Training lernten Probanden ein neues Gangmuster, das phasenverschoben war (Casile \& Giese, 2006). Dabei wurden die Augen der Probanden abgedeckt, sie erhielten nur verbales oder haptisches Feedback.

Das Training wirkte sich auf die Wahrnehmung in einem späteren Test aus und es wurden hohe Korrelationen zwischen der motorischen Beherrschung und der Wahrnehmungsleistung gefunden. Das bedeutet, dass die Probanden, welche die Bewegungen am besten erlernt hatten, auch im Wahrnehmungstest besser abschnitten. Die Ergebnisse weisen drauf hin, dass die rein motorische Erfahrung Einfluss auf Wahrnehmungsleistungen hat.

\subsubsection{Vorstellungen als abhängige Variable}

Eine Moderatorvariable, welche die Wirkung des Mentalen Trainings beeinflusst, scheint die Expertise zu sein. Bohan, Pharmer und Stokes (1999) untersuchten die Wirkung Mentalen Trainings in drei Fertigkeitsgruppen. Danach nehme die Effektivität des Mentalen Trainings bei zunehmender Fertigkeitsbeherrschung ab.

Lorey et al. (2009) haben den Einfluss der Körperposition und Perspektive auf die Vorstellungsfähigkeit mittels fMRT untersucht. Dabei wurden Aktivitäten im Gehirn bei der Vorstellung des Probanden entweder in der Erste-Person-Perspektive oder der Dritte-Person-Perspektive miteinander verglichen. Es zeigte sich, dass die Körperposition für die Vorstellung nur in der Erste-Person-Perspektive relevant war und dass allgemein motorische neuronale Areale in dieser Perspektive stärker aktiv waren. 
Dies wurde als „embodied nature of motor imagery“ (Lorey et al., 2009, S. 233) interpretiert.

Anhand des Mentalen Chronometrie-Paradigmas hat Munzert (in press) drei Gruppen (Badminton-Experten, eine mittlere Expertisegruppe und Novizen) untersucht. Sie sollten eine Bewegungsfolge im Badminton ausführen und sie sich vorstellen. Experten konnten die vorgestellte Zeitdauer genauer einschätzen. Dabei wurden tendenziell kürzere Bewegungssequenzen überschätzt und längere unterschätzt, was Munzert (ebd.) auf die speziellen Aspekte der mentalen Vorstellungsaufgabe in dieser Studie zurückführt. Die Korrelationen zwischen mentaler und tatsächlicher Ausführung sind bei dieser Sportart sehr gering. Dies wird darauf zurückgeführt, dass eine genaue zeitliche Repräsentation nicht erforderlich ist oder aufgrund der eher visuell geprägten Vorstellungsnatur beim Badminton diese Kapazitäten eher zurückgestellt werden.

Eine solche Studie ist für das Tennisspiel nicht bekannt. Zu erwarten wären signifikante Zusammenhänge zwischen Vorstellungen und Handlungsausführung, da zeitliche Aspekte eine größere Rolle spielen könnten.

Jansen und Heil (2010) untersuchten den Zusammenhang zwischen dem motorischen Entwicklungsstand und der Mentalen Rotationsfähigkeit bei 5- bis 6-jährigen Kindern (gemessen mit dem „Picture Mental Rotation Test“, einem Test für Mentale Rotation mittels Zeichnungen). Eine Regressionsanalyse mit den Variablen Intelligenz (gemessen mittles CPM: Coloured Progressive Matrices Test, einem non-verbalen Denktest) und die motorische Leistung gemessen mit dem MOT-Test für 4- bis 6-jährige Kinder ergab, dass diese Variablen unabhängige und bedeutsame Prädiktoren für die Leistung bei der Mentalen Rotation waren. Nur bestimmte Leistungen des MOT-Tests sind ein signifikanter Prädiktor in der Multiplen Regressionsrechnung (z.B. ist ein Indikator „sich durch einen Reifen winden“). Laut den Autoren stellen sie die Aspekte dar, die eine zeitliche und räumliche Koordination benötigen. Dabei ist zu beachten, dass zu keiner der drei Variablen Geschlechter-Effekte gefunden wurden. Der verwendete Test für die Mentale Rotation war ein sogenannter „paper-and-pencil“-Test mit Bildmaterial (z.B. ein Tiger). Hierbei fand im Gegensatz dazu Hahn (2010) Geschlechterunterschiede bei der Mentalen Rotation, insbesondere einen Vorteil bei Jungen bezüglich der Fehlerraten, nicht aber hinsichtlich der Rotationsgeschwindigkeit (vgl. Kap. 7.7.2).

Auch die Studie von Keller, Knoblich und Repp (2007) an Pianisten gehört in dieses Kapitel. Im Ergebnis erkannten Pianisten sich selbst besser als andere Spielpartner (siehe Hinführung zu den Fragestellungen im Kap. 3). 


\subsection{Vorstellung und Gedächtnis: Interferenzeffekte}

Baddeleys unvollständiges Arbeitsgedächtnismodell

Die Fähigkeit zur Verbindung des Bewegungswissens spielt eine bedeutende Rolle bei der Handlungsregulation (Jeannerod, 1995). So nimmt Jeannerod (ebd.) an, dass eine Kopie der verschiedenen Teilziele aus dem Gedächtnis dann aktiviert wird, wenn die Handlung gemäß einem Ziel verfolgt wird. Bei Störungen der Handlungsausführung kommt es zur internen Wiederholung dieser Repräsentationseinheiten. Hiermit postuliert Jeannerod (ebd.) einen Prozess, der auch schon im Modell des Arbeitsgedächtnisses von Baddeley (z.B. 1997) eine Rolle spielt: der sogenannte Rehearsal-Prozess. Hier wurden aber nur Annahmen über die Wiederholung verbaler Informationen integriert. Der Ort hierfür wurde "Artikulatorische Schleife“ (phonological loop) genannt. Baddeley (2012) fragte kürzlich in seinem Ausblick, inwieweit dieser Wiederholungsprozess auch für nicht verbales Material, wie zum Beispiel Musik oder Hintergrundgeräusche gelte. Bewegungen wurden bisher nicht berücksichtigt. Unklar ist auch, ob solch ein Wiederholungsprozess im visuo-räumlichen Skizzenblock vonstatten geht (Logie, 1995) oder ob es sich hierbei um einen aufmerksamkeitsbasierten Wiederbelebungsprozess („attentionally based refreshing“ (ebd., S. 23) handelt. Damit stellt sich das Modell als unvollständig dar.

\section{Zwei Thesen mit gleichen Vorhersagen aber unterschiedlichen Erklärungen}

Hier stehen zusammengefasst zwei Positionen nebeneinander, die sich auf unterschiedliche Faktoren beziehen, wenn es um den Einfluss von Vorstellungen auf die Wahrnehmung geht. Zum einen Eysenck (2012), der von inhaltlichen Faktoren ausgeht (These 1) und Ishai und Sagi (1997b), die von der Art des Gedächtnisses ausgehen (These 2).

Diese zwei unterschiedliche Thesen über die Ursachen von Interferenzen bei Wahrnehmung und Vorstellung werden im Folgenden erläutert: Unter Interferenzen werden hemmende Überlagerungen einer Gehirnfunktion durch weitere Gehirnaktivitäten verstanden, die gleiche oder ähnliche Gehirnareale, Repräsentationen oder Prozesse beanspruchen.

Bei der These 1 wird angenommen, dass der Inhalt von Mentalen Repräsentationen bestimmt, ob Vorstellungen und Wahrnehmungen sich gegenseitig stören oder das eine die andere erleichtert. Bei der These 2 wird angenommen, dass hierbei auch die Art des 
involvierten Gedächtnisses eine Rolle spielt.

Wie schon in Kap. 6.1 erwähnt, gibt es Gemeinsamkeiten zwischen Wahrnehmung und Vorstellung. Eysenck (2012) schreibt: „If visual perception and visual imagery involve similar processes, they should influence each other. There should be facilitative effects if the content if the perception and the image is the same but interference effects if the content is different" (Eysenck, 2012, S. 55). Vergleiche hierzu auch die Äußerungen von McGinn (2007) in Kap. 6.2.1 zu den qualitativen Unterschieden von Wahrnehmungen und Vorstellungen.

Diesen Erleichterungseffekt (facilitative effect) haben Pearson, Clifford und Tong (2008) im Rahmen eines binokularen Rivalitätsparadigmas gefunden. Dieses Paradigma verwendet farbige Balken bestimmter Orientierung, um beiden Augen jeweils einen unterschiedlichen Reiz zu zeigen (z.B. dem linken Auge einen horizontalen grünen und dem rechten Auge einen vertikalen roten Balken). Dabei ergibt sich das Phänomen, dass ein Muster dominanter als das andere wahrgenommen wird. Dann wird die binokulare Reizsituation (BR) hergestellt, diese wird durch einen dritten sich vorgestellten Reiz unterbrochen, wobei der sich vorgestellte Reiz entweder das dominante Muster oder das weniger dominante Muster dargestellt. Es ergibt sich der Befund, dass durch die Vorstellung des dominanten Musters auch eine tendenziell etwas höhere Wahrnehmungsschwelle dieses Musters in der BR erzielt wird; die Vorstellung des nicht dominanten Musters hat eine signifikante Erniedrigung der Schwelle des nicht dominaten Musters in der BR zur Folge. Als kritisch ist die geringe Fallzahl und der nicht signifikante Erleichterungseffekt zu bewerten. Wohl aber ergibt sich hier ein Trend zu einer Erleichterung der Wahrnehmung durch die Vorstellung bei gleichem Inhalt.

Ishai und Sagi (1997b) untersuchten Interferenzeffekte im Rahmen der These, ob das Kurz- bzw. das Langzeitgedächtnis dafür verantwortlich sei (vergleiche auch Ishai \& Sagi (1997a) in Kap. 6.2.2). In einem ersten Versuch wurden zunächst vertikale und horizontale Linien präsentiert, damit sie im LZG gespeichert werden. Hiernach wurden drei Bedingungen unterschieden:

a) Die Vorstellungsbedingung, bei der sich vertikale Linien vorgestellt werden sollten,

b) die Vorstellungsbedingung, bei der sich horizontale Linien vorgestellt werden sollten sowie

c) eine Kontrollbedingung, bei der die zuvor präsentierten Linien erkannt werden sollten.

Dabei zeigte sich eine orientierungsspezifische Interferenz, d.h. bei der Vorstellungs- 
bedingung mit den vertikalen Linien erhöhte sich die Wahrnehmungsschwelle im Vergleich zu den beiden anderen Bedingungen signifikant. Dies bedeutet, dass die Wahrnehmung der Linien in dieser Bedingung schwerer wurde. Zusätzliche Experimente sollten eine Reihe von Alternativerklärungen ausschließen: Ein abgeschwächter Interferenzeffekt wurde gefunden, wenn die Linien aus dem Kurzzeitgedächtnis generiert wurden. Dazu wurden folgende Bedingungen verglichen:

a) Wahrnehmungsbedingung: Es wurden zugleich vertikale und horizontale Linien präsentiert und die vertikalen Linien sollten erkannt werden. Nur die vertikalen Linien hatten dabei verschwommene Konturen. Diese Linienarten werden auch als sogenannte Gabor-Linien bezeichnet.

b) In der Vorstellungsbedingung sollten sich die Probanden vertikale Linien vorstellen, während sie horizontale Linien sahen. Nur die horizontalen Linien hatten verschwommene Konturen (Gabor-Linien).

c) Eine nicht näher beschriebene Kontrollbedingung.

Als Ergebnis erhöhte sich die Wahrnehmungsschwelle in der Vorstellungsbedingung im Vergleich zur Wahrnehmungsbedingung (siehe Abb. 5). Bei Beteiligung des Kurzzeitgedächtnisses, so die Autoren, reduzierte sich der Interferenzeffekt im Vergleich zum ersten Versuch (siehe oben). Bei der Beteiligung des Langzeitgedächtnisses traten vorstellungsinduzierte Interferenzeffekte auf die Wahrnehmung auf. Ishai und Sagi (1997b) schlussfolgerten auch für den Einfluss von Vorstellungen auf die Wahrnehmung, dass der Abruf visueller Informationen aus dem LZG die Wahrnehmung stört, während die Reizerkennung erleichtert wird, wenn hierzu Informationen aus dem KZG abgerufen werden.

Während Ishai und Sagi (1997b) vorrangig den Effekt von visuellen Vorstellungen auf die visuelle Wahrnehmung untersuchten und dabei Effekte der Störung oder der Erleichterung in Abhängigkeit vom Gedächtnis aufzeigten, soll es hier in erster Linie um mentale Ergänzungsleistungen in der visuellen Vorstellung unter Rückgriff auf motorische Repräsentationen gehen. Genauer untersucht wird die Rolle des Langzeitgedächtnisses auf die Wahrnehmung unvollständig präsentierter Bewegungsreize. 


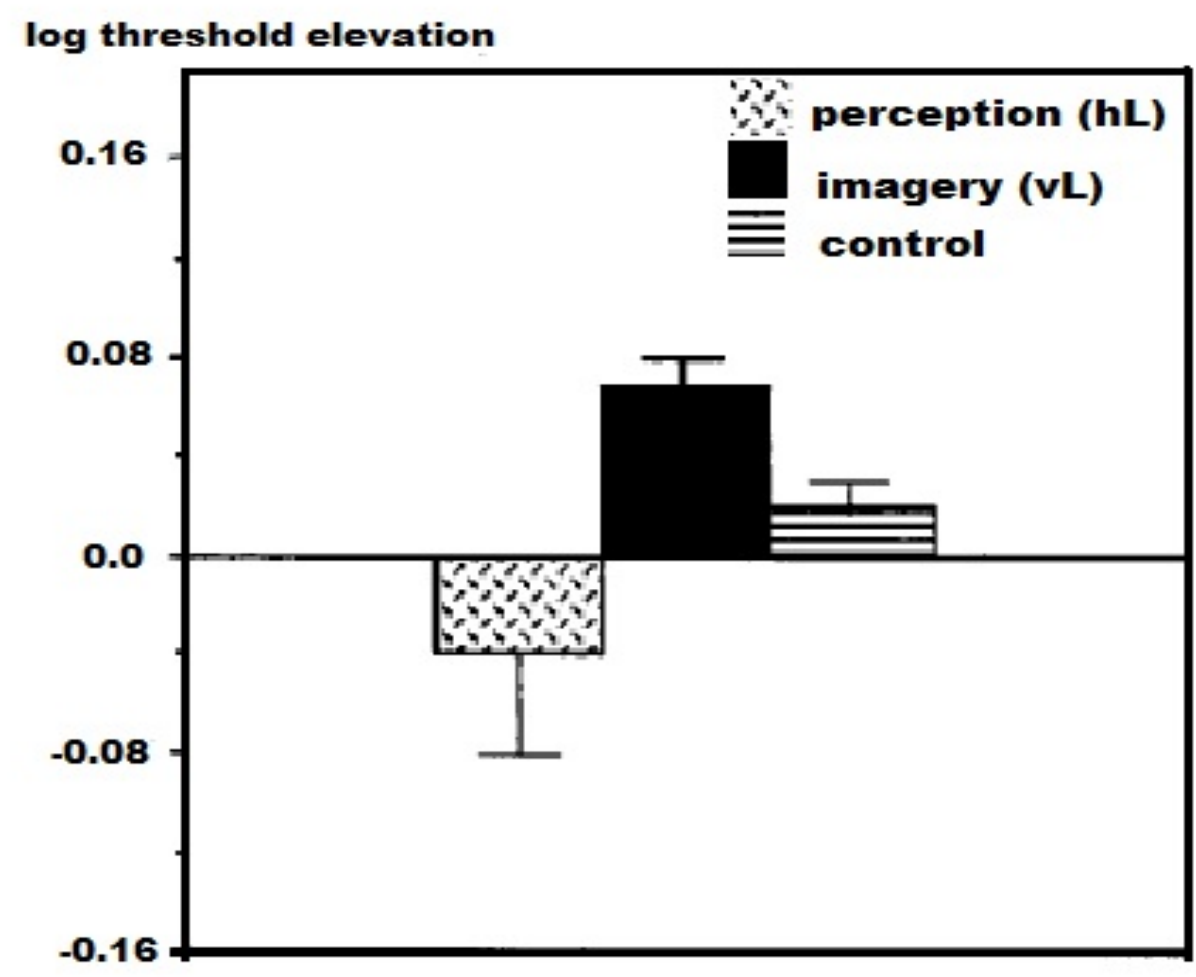

Abbildung 5: Ergebnisse des Experiments urspr. nach Ishai und Sagi (1997b, S. 737)

Visuell-räumliche Interferenzen

Um die Natur von Vorstellungen zu erforschen, benutzte Brooks (1968) eine Interferenzaufgabe.

\begin{tabular}{|l|c|c|c|}
\hline \multicolumn{1}{|c|}{ Reizmaterial } & \multicolumn{3}{|c|}{ Antwortformat } \\
\hline & $\begin{array}{c}\text { Zeigen } \\
\text { [in Sek.] }\end{array}$ & $\begin{array}{c}\text { Klopfen } \\
\text { [in Sek.] }\end{array}$ & $\begin{array}{c}\text { Sprechen } \\
\text { [in Sek.] }\end{array}$ \\
\hline Zeichnung (F) & 28,2 & 14,1 & 11,3 \\
\hline Satz & 9,8 & 7,8 & 13,8 \\
\hline
\end{tabular}

Tabelle 1: Ergebnisse des Experiments von Brooks (1968), Reaktionszeiten in Sekunden für die Klassifikation

Während Versuchspersonen sich Buchstaben vorstellen sollten, mussten sie gleichzeitig beurteilen, ob einzelne Wörter in einem Satz Substantive waren oder nicht. Die 
Antworten bezüglich dieser Sekundäraufgabe wurden in drei verschiedenen Formaten gefordert: Probanden sollten sie zeigen, klopfen oder sprechen.

Es zeigte sich, dass das Scannen von Buchstaben bzw. die Beantwortung der Frage, ob die Flächen des Buchstabens innen oder außen liegen, beim Antwortformat „zeigen“ länger dauerte als beim Aussprechen der Antwort. Hingegen dauerte eine Kategorisierung der Wörter am längsten beim „Sprechen“ und am schnellsten ging es beim „Zeigen“ (siehe Tab. 1). Dies wurde dahingehend interpretiert, dass ein Konflikt zwischen dem Scannen des Antwortblattes und dem Scannen einer mentalen Anordnung (Zeichnung von F) besteht und somit die Mentale Repräsentation der Vorstellungen eine Analogie zur physikalischen Anordnung hat.

Beruhen die Ergebnisse von Brooks (1968) tatsächlich auf einem Konflikt zwischen der Ausführung einer visuellen Zeigeaufgabe und dem Scannen einer visuellen Vorstellung? Baddeley (1976) differenzierte die Ergebnisse von Brooks: Er erweiterte Brooks Aufgabenstellung, indem gleichzeitig das Scannen des Buchstabens entweder mit einer Aufgabe mit eher räumlichen oder eher visuellen Anteilen verlangt wurde (Doppelaufgabe). Hierbei zeigten sich längere Reaktionszeiten beim Scannen der räumlichen Zweitaufgabe, weshalb auf räumliche Interferenz als Erklärung der Ergebnisse geschlossen wurde.

Die Aufgabe mit den räumlichen Anforderungen behinderte das Scannen des mentalen Bildes stärker als die visuelle Aufgabe. So wird geschlussfolgert, dass das Scannen mentaler Bilder durch Interferenzen behindert wird, wenn gleichzeitig räumliche Strukturen der Umgebung verarbeitet werden.

\section{Auch Handlungen beeinflussen Vorstellungen}

Es wird angenommen, dass mit der Vorstellung inkompatible Handlungen zu Störungen der Vorstellungen führen, während sie durch kompatible Handlungen erleichtert werden (Wexler, Kosslyn \& Berthoz, 1998). Der Versuch wird nachstehend erläutert:

In einem Doppelaufgaben-Paradigma wurde von Wexler et al. (ebd.) folgendes angenommen: "[...] visuomotor anticipation is the engine that drives mental rotation. Mental rotation would thus recruit motor planning and anticipation, but not the cortical and subcortical mechanisms responsible for the execution of movement" (Wexler, Kosslyn \& Berthoz, 1998, S. 79). Deshalb prüften sie, ob die Bewegungsausführung und Vorstellung (als Mentale Rotation operationalisiert) miteinander interferieren. So wurde gleichzeitig eine Bewegungsaufgabe und eine Mentale Rotationsaufgabe gestellt. Bei ersterer mussten die Probanden einen Joystick entweder in eine mit der Mentalen 
Rotation kompatiblen Richtung drehen oder in die entgegengesetzte Richtung, wobei sie den Joystick nicht sehen konnten. Die Geschwindigkeit war vorher einstudiert und variierte in einem Teilexperiment. Bei der Vorstellungsaufgabe sollte beurteilt werden, ob eine abstrakte Figur eine rotierte oder gespiegelte Abwandlung des Originals war. Anders als bei der ansonsten gleich aufgebauten Studie von Cooper und Shepard (1973) wurden die abstrakten Objekte nicht gleichzeitig, sondern hintereinander gezeigt. Ein Pfeil gab die Lage der zweiten Figur an. Als Ergebnis zeigte sich eine stärkere Interferenz beider Aufgaben, wenn die Bewegungsrichtung des Joysticks nicht kompatibel mit der Richtung der Mentalen Rotation war. Nicht nur die Richtung der Bewegung, sondern auch die Geschwindigkeit hatte Einfluss auf die Interferenz: Bei größerer Geschwindigkeit der Bewegungsausführung war auch die Vorstellung schneller. Es wurde außerdem eine V-förmige Reaktionskurve gefunden: Die geringsten Reaktionszeiten entstanden bei einem Mentalen Rotationswinkel von $45^{\circ}$ (abweichend von Cooper und Shepard (1973), als die kleinsten Reaktionszeiten im Winkel von $0^{\circ}$ festgestellt wurden). Diese Ergebnisse interpretierten die Autoren im Sinne ihrer Hypothese, dass Bewegungsausführung und Vorstellung gemeinsame Mechanismen zur Informationsverarbeitung nutzen.

Bei vorhandener Interferenz muss von gleichen neurologischen Strukturen ausgegangen werden; dies beinhaltet auch solche Strukturen, welche die Bewegungsausführungen steuern. Das ist widersprüchlich, da sie im Zitat oben ausgeschlossen werden.

Ebenfalls sollten Probanden bei einem Doppelaufgaben-Experiment fünfzehn mal eine Bewegung von unterschiedlicher Weite (30 versus $60 \mathrm{~cm}$ ) ausführen und diese nach einem Behaltensintervall nachahmen. Johnson (1982, zit. nach Engelkamp \& Zimmer, 2006) zeigte dabei, dass die gleichzeitige Ausführung von konkurrierenden Bewegungen (real und in der Vorstellung) jeweils zu einer Über- oder Unterschätzung der Reproduktion der ursprünglichen Bewegungsweite führt (je nachdem, ob die Konkurrenzbewegung länger oder kürzer war). Das Zählen und die Vorstellung der gleichen Bewegungsweite spielen hingegen keine Rolle, sie stören die Aufgabe nicht.

Ob die Faktoren Inhalt oder Gedächtnis oder beide für solche Effekte wie Interferenz oder Erleichterung der Wahrnehmung von Objekten verantwortlich sind, ist ungeklärt. Diese Dissertation wird den Faktor Gedächtnis näher beleuchten. 


\subsection{Vorstellung und Verkörperung}

Hier wird ein Ansatz mit der Hauptthese dargestellt, dass Athleten besondere Wahrnehmungsfähigkeiten erlangen und diese auch auf das Erlernen von motorischen Fähigkeiten zurückwirken. Im Gegensatz zu Ishai und Sagi (1997b) soll das gespeicherte (motorische und visuelle) Wissen positive Auswirkungen auf die Bewältigung der in den Versuchen dieser Dissertation gestellten Vorstellungs- und Wahrnehmungsaufgabe haben.

Im Rahmen des „embodied cognition“- oder auch „embodied perception“- Ansatzes wird der Einfluss motorischen Wissens und Könnens auf die Wahrnehmung und umgekehrt der Wahrnehmung auf das motorische Wissen und Können erforscht. Dies steht im Gegensatz zu traditionellen Auffassungen von Wahrnehmung. Demnach ist diese von der körperlichen Verfassung unabhängig (z.B. Marr, 1982).

Theoretische Modelle und Theorien der „embodied perception“ sehen das visuelle System nicht als Symbolverarbeitungssystem, sondern eingebettet in den Körper. Dabei bestimmen z.B. körperliche Zustände oder Handlungsmöglichkeiten die visuelle Wahrnehmung (Shiffrar \& Heinen, 2010). Die Möglichkeit, Bewegungen zu initiieren, beeinflusst außerdem die Wahrnehmung dieser Bewegung (Wilson, 2001, zit. nach Shiffrar \& Heinen, 2010). „Visuelle Wahrnehmungen verändern sich dabei als Funktion des Grades, inwieweit das motorische System eines Beobachters eine beobachtete Bewegung repräsentieren und reproduzieren kann“ (Shiffrar \& Heinen, 2010, S. 133).

Die Autoren vertreten, anders als allgemeine Modelle zur Wahrnehmung, die Auffassung, dass Wahrnehmung nicht ohne den Körper geschieht und dass diese von bestimmten Faktoren beeinflusst werden kann:

„Empirische Ergebnisse deuten in diesem Kontext darauf hin, dass die Wahrnehmungsbildung der physikalischen und sozialen Umwelt davon abhängt, inwieweit sich der Körper von Beobachtern in dieser Umwelt bewegen kann (Bewegungsmöglichkeiten), sich bereits bewegt hat (Bewegungserfahrung), sich aktuell bewegt (Bewegungszustand) und sein Bewegungssystem repräsentiert (Körperschema).“ (Shiffrar \& Heinen, 2010, S. 140)

Daraus werden von Shiffrar und Heinen (2010) sinngemäß folgende Hypothesen abgeleitet:

1. Visuelle und motorische Erfahrung korrelieren mit der Aktivität des motorischen 
Systems.

2. Die Wahrnehmung bewegter Objekte unterscheidet sich von der Wahrnehmung menschlicher Bewegungsabläufe.

3. Die Wahrnehmung wird von spezifischen motorischen Fertigkeiten beeinflusst.

4. Die motorischen Fertigkeiten beeinflussen die Wahrnehmung.

So belegen Shiffrar und Heinen (2010) viele der Hypothesen mit den speziellen Fähigkeiten der Athleten, deren gute körperliche Verfassung sowie gute sportliche Fertigkeiten sich positiv auf die Wahrnehmung auswirken. Aufgrund der Wahrnehmungsähnlichkeit von Vorstellungen sollten viele der Thesen auch für diese gelten.

Im Folgenden werden Befunde zu den jeweiligen Thesen dargestellt:

\section{These 1: Zusammenhang der Aktivität des motorischen Systems mit der Erfahrung}

Die folgenden Befunde deuten darauf hin, dass „[...] die Aktivität des motorischen Systems mit dem Grad an motorischer Erfahrung mit einer spezifischen, beobachteten Bewegung korreliert" (Shiffrar \& Heinen, 2010, S. 135). Dabei ist anzumerken, dass die motorische Erfahrung immer auch mit visueller Erfahrung verbunden ist.

Casile und Giese (2006) haben in ihrem Lernexperiment den Erwerb einer neuen Bewegung des Oberkörpers und dessen Einfluss auf die Wahrnehmung von sogenannten „point-light-Läufern“ gemessen. Es wurde von Casile und Giese (2006) ein motorisches Training ohne visuelles Feedback durchgeführt. Sie verglichen die Leistungen mit einer Kontrollgruppe, die rein visuelles Training erhielt. Dies wurde deshalb derart konzipiert, da motorisches und visuelles Training in der Realität nur gekoppelt vorkommen. Als Ergebnis konnten Casile und Giese (ebd.) ihre Hypothese bestätigen: Es wurden nur motorisch trainierte Bewegungsmuster im Vergleich zu motorisch untrainierten erfolgreich erkannt. Es korrelierte die Genauigkeit der Ausführung der gelernten Bewegungen mit der visuellen Wiedererkennung. Demnach beeinflussen motorische Programme selektiv die visuelle Wiedererkennung.

Proffitt et al. (1995) zeigten, dass die Wahrnehmung von physikalischen Eigenschaften vom körperlichen Zustand (z.B. der Bewegungsfähigkeit oder dem Ermüdungszustand) abhängt. So wird ein Berg steiler wahrgenommen, wenn sich die Person auf dem Berg befindet oder zuvor einen Ausdauer-Lauf absolviert hat.

Spiegelneurone feuern des Weiteren nur bei Affen, wenn es sich um Bewegungen von Primaten, nicht von Maschinen handelt (Rizzolatti et al., 2001, zit. nach Shiffrar \& Heinen, 2010). Dies zeigt, dass die spezielle Erfahrung, die eine Spezies mit ihren 
Bewegungen hat, das motorische System aktiviert.

Die folgende Studie verdeutlicht, dass dies nicht nur in Bezug auf die Bewegungen einer Spezies gilt, sondern auch für Handlungen, die ein Individuum ausführen kann: So konnten Buccino et al. (2004) zeigen, dass Handlungen, die vom Beobachter ausgeführt werden konnten, im Gegensatz zu von ihnen nicht ausführbaren Handlungen, die Aktivität von Spiegelneuronen stärker beeinflussen (zit. nach Shiffrar \& Heinen, 2010). Vergleiche dazu Calvo-Merino et al. (2005) sowie Calvo-Merino et al. (2006) (siehe Kap. 7.5.2).

\section{These 2: Die Wahrnehmung bewegter Objekte versus der Bewegung von Menschen}

Eine weitere Hypothese dieser theoretischen Orientierung ist die, dass die visuelle Wahrnehmung imitierbarer Reize auf eine andere Art erfolgt als die nicht imitierbarer Stimuli (Shiffrar \& Heinen, 2010). Zum Beispiel kann eine sich bewegende Person anders wahrgenommen werden als ein fliegendes Objekt. Aus der Sichtweise der „embodied perception“ unterscheidet sich somit die Wahrnehmung bewegter Objekte von der von Menschen (Shiffrar \& Heinen, 2010). Vergleiche dazu auch Johansson (1973).

Werden etwa Standbilder menschlicher Bewegungen nacheinander gezeigt, so werden diese bei einer geringen Bildwechselfrequenz - ähnlich der Bilder von Objekten - entlang eines kürzest möglichen Bewegungspfades wahrgenommen. Werden Standbilder von menschlichen Bewegungen in "Echtzeit“ gezeigt, werden sie zudem anhand der biomechanischen Gesetze der Bewegungsproduktion wahrgenommen, nicht so Standbilderfolgen von Objektbewegungen (Shiffrar \& Freyd, 1990, 1993, zit. nach Shiffrar \& Heinen, 2010).

\section{Thesen 3 und 4: Die besonderen Fähigkeiten von Athleten}

Shiffrar und Heinen (2010) wiesen darauf hin, dass Athleten, die besondere motorische Fähigkeiten haben, auch besondere Wahrnehmungsfähigkeiten besitzen (z.B. Abernethy, 1990; Kioumourtzoglou et al., 1998; Williams \& Davis, 1998, zit. nach Shiffrar \& Heinen, 2010). Dabei seien diese nur zum Teil der perzeptuellen Expertise geschuldet (hier gemeint: vorheriges visuelles Lernen) vor allem aber der motorischen Expertise.

Weiterhin haben Untersuchungen gezeigt, dass die Ausführung von Bewegungen durch den Beobachter die Wahrnehmung anderer Personen beeinflusst (Jacobs \& Shiffrar, 2005; Reed \& McGoldrick, 2007, zit. nach Shiffrar \& Heinen, 2010). 
Zudem wurde festgestellt, dass die Wahrnehmung von den konditionellen Fähigkeiten des Beobachters abhängig ist. Beim Gehen auf einem flachen Laufband werden die Personen anders wahrgenommen als auf einem steilen Band. Dies wird zusätzlich durch den Faktor Kondition moderiert. Personen mit besserer Kondition schätzen die Geschwindigkeit auf dem Laufband eher langsam ein als weniger fitte, die sie als schnell wahrnehmen (Jacobs \& Shiffrar, 2005, zit. nach Shiffrar \& Heinen, 2010).

Die visuo-motorische Expertise kann dazu führen, die Intentionen anderer Spieler, z.B. im Tennis, besser vorauszusagen (Shiffrar \& Heinen, 2010). Für die Sportart Tennis gibt es Untersuchungen, die darauf hinweisen, dass es nötig ist, um die Flugbahn des Balls vorherzusagen, eine Analyse des gesamten Körpers des Gegners durchzuführen (Huys et al., 2009, zit. nach Shiffrar \& Heinen, 2010). Für die Sportart Basketball konnten Hinweise für die Benutzung dynamischer Reize für das Erkennen von Täuschungsmanövern gesammelt werden (Sebanz \& Shiffrar, 2009, zit. nach Shiffrar \& Heinen, 2010). Dabei sollten Videosequenzen, „point-light-Aufnahmen“ oder statische Bilder vor einem echten oder vorgetäuschten Abwurf des Balls hinsichtlich einer Täuschung klassifiziert werden. Bei den Bedingungen mit dynamischen Stimuli waren die Spieler am effizientesten. Bei Badmintonspielern wurde dabei eine erhöhte Aktivität in Gehirnregionen gefunden, die mit der Ausführung von Handlungen in Verbindung gebracht wird (Wright et al., 2010, zit. nach Shiffrar \& Heinen, 2010).

Eine weitere Studie stützt die Vorhersage, dass motorische Expertise (im Vergleich zur visuellen Expertise) die Wahrnehmungssensitivität hinsichtlich bestimmter Handlungen erhöht: Dazu haben Aglioti et al. (2008, zit. nach Shiffrar \& Heinen, 2010) Basketballprofis, Basketballtrainer und Sportjournalisten gebeten, anhand von Videos von Korbwürfen die Treffgenauigkeit vorherzusagen. Nur die Basketballprofis, die sowohl über die motorische als auch über die visuelle Erfahrung verfügten, waren den anderen Gruppen überlegen. Im Vergleich zu den Journalisten wiesen die Profis eine klarer differenzierte Aktivierung auf. Dies wurde anhand der transkraniellen Magnetstimulation, die die kortikale Erregbarkeit messen soll, festgestellt. 


\subsection{Zusammenhang von Vorstellung und motorischen Fertigkeiten in der Kindheit}

Die Variable Alter ist mit der Variablen Erfahrung konfundiert. Das bedeutet, dass ältere Kinder auch mehr Erfahrung mit einer Bewegung haben als jüngere. Kritisch ist anzumerken, dass Kinder aber trotzdem keine Bewegungsexperten sein müssen. Ihre motorischen Fertigkeiten müssen trotz eines höheren Alters und dem jahrelangen Erlernen einer Sportart nicht exzellent sein. Dennoch sind die Variablen Alter und Erfahrung möglicherweise wichtige Determinanten der Beziehung zwischen Vorstellungen und Bewegungsfertigkeiten. Wobei auch anzumerken ist, dass die Variable Alter eine Beschreibungsvariable darstellt, d.h. sie kann Ursachen nicht erklären.

Es gibt nur sehr wenige Studien, die den Zusammenhang von Vorstellungen und motorischen Fertigkeiten in der Kindheit untersuchen.

Es fanden etwa Caeyenberghs et al. (2009a) einen mit dem Alter stärker werdenden Zusammenhang zwischen Vorstellungen und motorischen Fertigkeiten. Sie untersuchten altersabhängige Entwicklungsvorgänge zum Thema mentale Vorstellungen sowie die Beziehung zwischen Vorstellungen und Bewegungsfertigkeit. Sie maßen motorische und visuelle Vorstellungen bei Kindern im Alter zwischen 7 und 12 Jahren. Dies testeten sie durch folgende Aufgaben: Die Zeigeaufgabe umfasste eine tatsächliche und eine vorgestellte Hin- und Herbewegung zwischen einem Startpunkt in der Mitte und einem Halbkreis darüber angeordneter Ziele. Diese Ziele waren pro Durchgang in unterschiedlicher Größe dargestellt, was eine Variation der Schwierigkeit bedeutete. Bei der Handrotationsaufgabe (Erste-Person-Perspektive, motorische Vorstellung) wurden Hände in zufälliger Reihenfolge rotiert und zwar in $45^{\circ}$-Schritten. Die Aufgabe war es, die eigene Hand so in der Vorstellung umzudrehen, dass sie mit der präsentierten Hand übereinstimmt, um dann die Seitigkeit der Hand zu beurteilen. Bei der visuellen Vorstellungsaufgabe wurden Buchstaben in verschiedenen Winkeln rotiert und es sollte entschieden werden, ob die Reize rotiert oder gespiegelt waren. Weiterhin wurde die motorische Fertigkeit (Grob- und Feinmotorik) mittels des „McCarron Assessment of Neuromuscular Development" (McCarron, 1997) erhoben, um eine Beziehung von Vorstellungsaktivitäten und Bewegungsausführung zu erheben. Die Ergebnisse wiesen auf Altersunterschiede bei Bewegungsfertigkeiten, motorischen Vorstellungen und visuellen Vorstellungen hin. Signifikante Verbesserungen der motorischen Vorstellungs- 
fähigkeit ließen sich so ab 7-8 Jahren nachweisen (hier die jüngste Altersgruppe). Die Beziehung zwischen motorischer Vorstellung und Bewegungsausführung wurde mit dem Alter stärker. Hingegen gab es zu keinem Zeitpunkt eine Beziehung von visueller und motorischer Fertigkeit, wohl aber von motorischer Vorstellung und Bewegungsfertigkeit. Die Autoren interpretieren dies als Hinweis darauf, dass motorische Vorstellungen eigenen Entwicklungsgesetzen folgen als visuelle Vorstellungen. Kritisch ist anzumerken, dass es sich hierbei nicht um eine Längsschnittuntersuchung handelt. Außerdem könnte der Befund durch die besser werdende Beherrschung des Buchstabenmaterials (visuell und motorisch) erklärt werden. Es sollten auch noch jüngere Kinder in die Studie mit eingeschlossen werden. Zudem wurden australische Kinder untersucht, was kulturelle Einflüsse möglich macht.

\subsection{Vorstellung und Alter}

Ab wann können Kinder Vorstellungen erzeugen? Es stellt sich auch die Frage nach einem geeigneten Messinstrument hierfür.

Piaget und Inhelder (1971) nahmen an, dass Kinder, welche jünger als sieben Jahre sind, nur statische Vorstellungen erzeugen könnten, es sei ihnen nicht möglich, Bewegungen und Transformationen zu repräsentieren sowie Zukünftiges zu antizipieren. Das Voroperatorische Stadium im Rahmen der Theorie Piagets ist durch die Zentrierung auf Zustände gekennzeichnet, bei dem die Transformationen, die Objekte verändern, nicht als solche wahrgenommen würden, sondern das Endergebnis würde als neuer Zustand genommen und beurteilt (Oerter \& Montada, 1995). Erst mit 7-8 Jahren könnten nach Piaget und Inhelder (1971) dynamische Vorstellungen (die einfache Handlungsfolgen in der Zukunft einschließen können) gebildet werden, wenn die Kinder das Stadium der konkreten Denkoperationen erreichen.

\subsubsection{Bilderselektionstest}

Von Chevalier et al. (1995) wurde ein Test der Bewegungsvorstellung, ursprünglich von anderen Kollegen verwendet, adaptiert. Dabei sollten 13 Bewegungen vor einem Spiegel ausgeführt und sich vorgestellt werden, um dann diejenigen aus 4 Bildern auszuwählen, die der Vorstellung am ähnlichsten waren. Im Ergebnis wurde festgestellt, dass bereits 6jährige Kinder Vorstellungen erzeugen können. Hier stand die Frage nach kinästhetischen Vorstellungen im Vordergrund. Allerdings wäre es auch möglich, ohne tatsächliche Vorstellungen eine Auswahl aus 4 Bildern zu treffen, quasi per Zufall. 
Manidi-Faes (1996) fand heraus, dass sich die Vorstellung einer Bewegung nicht konstant entwickelt. Hierzu sollten Jungen im Alter von 6 bis 10 Jahren vor und nach der Ausführung einer Rolle vorwärts fehlerhafte Zeichnungen dieser Rolle erkennen. Die Ergebnisse zeigen einen Zusammenhang zwischen der Bildung der Vorstellung und dem Alter: es ergaben sich strategische Änderungen bei Vorstellungsleistungen bei Kindern im Alter von 6, 8 und 10 Jahren im Turnen insofern, als dass bei jüngeren und den ältesten Kindern dieser Gruppen Fehler in den Zeichnungen erkannt wurden, während diese Fähigkeit bei Kindern im Alter von 8 Jahren gestört schien. Außerdem hatte eine besonders große Anzahl von Übungswiederholungen bei den ältesten Kindern den größten Effekt.

Nach Ansicht der Autorin dieser Dissertation gibt es hier Hinweise auf strategische Veränderungen beim Einsatz von Vorstellungen. Kritisch ist anzumerken, dass bei einer quasi-experimentellen Studie keine Kausalaussagen möglich sind. Die Ergebnisse weisen auf zahlreiche Phasen hin, die nicht immer kontinuierliches Wachstum und Entwicklung beinhalten müssen.

\subsubsection{Mentale Rotation}

Marmor (1975) kritisierte Piaget und Inhelder (1971) hinsichtlich dessen, dass ein Kind zwar die zu untersuchende Fähigkeit besitzen könnte (Kompetenz), sie aber nicht im geforderten Antwortformat zeigen kann (Performanz). Auch nannte sie das Aufgabenverständnis als einen weiteren Störfaktor. Deshalb wurde in ihrer Studie eine andere Aufgabe, die von Shepard und Mitarbeitern entwickelt wurde, benutzt und leicht verändert: Hierbei sollten zwei zur selben Zeit gezeigte zweidimensionale Pandabären hinsichtlich der Gleichheit beurteilt werden. Die Vergleichsfigur war entweder ein Spiegelbild oder rotierte. Shepard und Metzler (1971) zeigten, dass die Reaktionsgeschwindigkeit linear mit dem Rotationswinkel des Objekts anstieg. Als Resultat ergab sich dieses Ergebnismuster auch bereits bei 5-jährigen Kindern. Dieses Ergebnis konnten Kosslyn, Margolis, Barrett, Goldenknopf und Daly (1990) bestätigen.

Kinder und Erwachsene wurden von Estes (1998) untersucht, ob sie sich bewusst sind, mentale Vorstellungen (bei der Mentalen Rotation) einzusetzen. Dabei konnte er zeigen, dass die Gruppe der 4- und 5-jährigen Kinder sehr heterogen war und nur die Kinder, welche die Aufgabe über dem Zufallsniveau lösen konnten, nannten auch mentale Vorstellungen als verwendete Technik. Bei 6-jährigen Kindern ist das Verhalten schon 
entsprechend der von Erwachsenen und zwar bezüglich der spontanen Nutzung und der Bewusstheit von mentalen Vorstellungen.

Kail, Pellegrino und Carter (1980) untersuchten Entwicklungsveränderungen bei der Mentalen Rotation bei Dritt-, Viert- und Sechstklässlern und einer Kontrollgruppe von Studenten. Das Alter betrug durchschnittlich 8;6, 9;2 und 11;6 Jahre. Dabei fanden sie heraus, dass die Geschwindigkeit der Mentalen Rotation mit dem Alter steigt und dass bekannte Reize (Zahlen) schneller rotiert wurden als unbekannte (Symbole). Ob möglicherweise jüngere Kinder die ganze Figur rotierten, ältere Kinder und Erwachsene jedoch nur Teile der Figur, bedarf weiterer Forschung. Es würden, so die Autoren, viele Informationsverarbeitungsprozesse automatisiert ablaufen (z.B. die visuelle Suche und der Informationsabruf), welches eine mögliche Erklärung der Ergebnisse wäre.

Funk, Brugger und Wilkening (2005) nutzten eine Mentale Rotationsaufgabe, die verschiedene Handpositionen beinhaltete, um bei 5- und 6-jährigen Kindern sowie bei Erwachsenen Vorstellungsprozesse zu untersuchen. Dabei hatten die Probanden die Aufgabe, auf Fotos von Händen zu bestimmen, ob eine rechte oder linke Hand abgebildet war. Die Hände wurden vor- und rückseitig mit jeweils 4 verschiedenen Rotationswinkeln präsentiert. Die Probanden sollten nun die verschiedenen Positionen erkennen: mit den Händen vorder- oder rückseitig (unsichtbar) unter eine Abdeckung abgelegt. Als Ergebnis konnten sie zeigen, dass sowohl Erwachsene als auch die Kinder länger brauchten, wenn die Position der eigenen Hände zur Position der Hände auf dem Foto ungünstig war. Es zeigte sich zusammengefasst als Hauptergebnis, dass die Hände bei Kindern und Erwachsenen am schnellsten erkannt wurden, die mit der eigenen Handposition übereinstimmten. Schon Kinder in diesem Alter sind fähig, Mentale Rotationen durchzuführen, nicht nur um die Gleichheit, sondern auch die Seitigkeit von Händen zu bestimmen. Die Forscher schlussfolgerten daraus, dass motorische Prozesse bei Kindern ( 5 und 6 Jahre) stärker an Vorstellungsprozessen beteiligt sind als bei Erwachsenen. Die Autoren argumentierten, dass eine stärkere Kopplung von sensomotorischen und visuellen Prozessen bei Kindern gegeben sei (siehe auch Bertenthal \& Clifton; 1998, Piaget, 1954; Rosenbaum, Carlson \& Gilmore, 2001) und die motorische Aktivität die Grundlage aller intellektuellen Fähigkeiten bilde. Letzteres wird hier auch vertreten. Es bleibt zu fragen, wieso diese Erklärung nur für Kinder gelten soll, wenn die Ergebnisse bei Erwachsenen ähnlich waren. Kritisch ist außerdem anzumerken, dass es womöglich bei den Erwachsenen einen Deckeneffekt gegeben 
haben könnte.

\subsubsection{Chronometrie-Paradigma}

Molina, Tijus und Jouen (2008) untersuchten 80 Kinder (Altersgruppen: 5 und 7 Jahre), um deren Fähigkeit zu testen, ein motorisches Vorstellungsbild zu erzeugen. Sie nutzten hierbei das Chronometrie-Paradigma. Dabei wird die Dauer von tatsächlicher und mentaler Vorstellung verglichen. Aufgabe war es eine Puppe von einem Ort zum anderen zu bringen (mental versus tatsächlich) unter zwei Bedingungen: keine Informationen über das Gewicht der Puppe oder die Information, dass sie schwer sein sollte. Ergebnis war eine signifikante Korrelation zwischen tatsächlicher und vorgestellter Bewegung nur bei den 7-Jährigen und zwar bei der Bedingung mit der Information über das Gewicht. Die Autoren schlussfolgerten, dass es Prozesse für das Sich-Vorstellen aus einer egozentrischen Perspektive (Erste-Person-Perspektive) bei 7-Jährigen gibt. Gabbard (2012) kritisiert, dass es für die 5-Jährigen möglicherweise Schwierigkeiten gibt, das Wort „schwer“ zu verstehen oder es sich vorzustellen.

\subsubsection{Sonstige Methoden}

Wenn sich dreieinhalbjährige oder ältere Kinder eine Lehrerin von zu Hause aus vorstellten, wie diese sich auf ihrem Platz in der Vorschule befindet, dann waren die Kinder besser, wenn sie tatsächlich auf die vorgestellte Lehrerin zugehen sollten als wenn sie sich die Bewegung zur Lehrerin nur vorstellten (Rieser, Garing und Young, 1994) . Dies interpretierten die Forscher als Hinweis für dynamische Vorstellungen in der Kindheit (siehe auch Frick, Daum, Wilson und Wilkening (2009) weiter unten).

Von Kosslyn, Margolis, Barrett, Goldknopf und Daly (1990) wurden Unterschiede bei 5-, 8- und 14-jährigen Kindern und Erwachsenen hinsichtlich verschiedener Maße für visuelle Mentale Vorstellungen verglichen: Bildgeneration, Bildbereithaltung, Scannen und Rotation. Während bei der Generation eines Vorstellungsbildes getestet wurde, ob eine Person überhaupt in der Lage ist, eine Vorstellung zu erzeugen, wurde beim Bereithalten geprüft, wie lange es im Arbeitsspeicher bereitgehalten werden konnte. Das Scannen wurde beispielsweise bei einem mentalen Größenvergleich erforderlich („Ist eine Maus größer als ein Elefant?") und die Rotation stellte eine Aufgabe dar, die noch heute für dynamische Bewegungsvorstellungen genutzt wird. Dabei werden Objekte in rotierter Weise präsentiert und die Aufgabe ist es zu entscheiden, ob ein anderer Reiz 
nebenan das gleiche Objekt ist oder nicht. Die Resultate ergaben, dass alle Altersgruppen Vorstellungen bilden konnten, so dass die Autoren nicht von sich spezialisierenden Vorstellungsfähigkeiten ausgingen. Allerdings hatten die 5-Jährigen Probleme beim Scannen, Rotieren und beim Vorstellungen generieren. Gut waren sie hingegen bei der Bereithaltung mentaler Bilder.

Frick, Daum, Wilson und Wilkening (2009) untersuchten mit einer Wasserstand-Aufgabe (water-level task), ob visuelle oder motorische Hinweise zu einer Bewegung die dynamische Vorstellungen bei Kindern im Alter von 5, 7 und 9 Jahren und Erwachsenen positiv beeinflussen können. Dabei sollten die Probanden ein Glas bei bekanntem, aber imaginärem Füllstand, so kippen, dass das Wasser fast heraus lief. Als Ergebnis zeigte sich bei den jüngeren Kindern (mit 5 Jahren), dass beim tatsächlichen Kippen mit der Nutzung des visuellem Feedbacks gute Erfolge erzielt wurden, aber bei der Vorstellungsaufgabe die Leistung eher schlecht war.

Es wurde geschlussfolgert, dass diese jüngeren Kinder bereits motorische Vorstellungen nutzen und sie dabei (eigenes) motorisches Feedback eher nutzen als ältere Kinder und Erwachsene. Letzteren halfen eher visuelle Hinweise. Wulf und Prinz (2001) zeigten etwa anhand des Golfspiels, dass die Lenkung der Aufmerksamkeit auf die Effekte von Bewegungen eine bessere Bewegungsleistung erzeugt. Dies wurde damit begründet, dass eine innere Aufmerksamkeit mit automatischen Kontrollprozessen des Arbeitsgedächtnisses in Konflikt gerät. Womöglich ist dies bei den jüngeren Kindern ebenfalls der Fall. Die Aufmerksamkeit auf innere Prozesse (Bewegungsgefühl) konfligiert hier mit der Aufgabenlösung.

\subsubsection{Fazit}

Ab dem Alter von 5 Jahren können Kinder Vorstellungen generieren (Marmor, 1975, Kosslyn et al., 1990). Mit anderen Methoden kann die Verwendung von Vorstellungen ab dem Alter von dreieinhalb Jahren gezeigt werden (Rieser et al., 1994). Es konnte nachgewiesen werden, dass bereits 6-jährige Kinder Mentale Rotationen durchführen können, um die Seitigkeit von Händen zu beurteilen. Die Kompetenzen von Kindern unterscheiden sich hinsichtlich der geringeren Vielfalt und Genauigkeit sowie in der Nutzung verschiedener Informationen (egozentrisch-motorische versus visuelle Hinweise) zu der von Erwachsenen. Es gibt außerdem Hinweise auf eine getrennte Entwicklung von visuellen und motorischen Vorstellungen. 
Diese Forschungsergebnisse sind die Basis dieser Arbeit. Ohne die Fähigkeit zur Vorstellung von Bewegungen wäre diese Untersuchung nicht möglich. Die Fähigkeit junger Kinder könnte bei der Imitation von Bewegungen helfen bzw. beim Erwerb sportlicher Fertigkeiten eine Rolle spielen. Wichtig ist hierbei auch, dass die präsentierte Vorlage, die es zu imitieren gilt, nicht rotiert wird, damit es gute Nachahmungsmöglichkeiten gibt. Beispielsweise sollte der Trainer, der einen Griff beim Schlag erklärt, zum Kind gehen und es nicht seitenverkehrt einweisen. 


\subsection{Vorstellung und Vorwissen}

Zunächst soll eine grundsätzliche Frage beantwortet werden: Wie unterscheiden sich Experten und Novizen? Dazu hat Huber (1997) in seiner Studie 4 Experten und 4 Novizen beim Springen vom Sprungbrett mittels Auswertung von Aussagen nach dem Sprung untersucht. Er stellte fest, dass die Konzepte der Experten komplexer waren als die der Novizen. Sie enthielten etwa eine größere Anzahl von Merkmalen und Verknüpfungen. Auch war der Fortschrittsgrad zur Diskriminierung und Prozeduralisierung größer.

Kritisch anzumerken ist die geringe Zahl der Probanden dieser Studie. Auch könnten die Ergebnisse dadurch erklärt werden, dass sich Experten im Laufe des Übens eine verbesserte Verbalisierungsfähigkeit angeeignet haben als Novizen. Denn wenn ein Trainer eine Bewegung zeigt, begleitet er das Zeigen auch mit Worten. Außerdem wird während der Nachahmung von Bewegungsabläufen möglicherweise verinnerlichtes Sprechen angewandt.

Es folgen Studien zu Vorstellungen und Expertise im Speziellen:

Naito (1994) nutzte den von Nishida et al. (1986, zit. nach Naito, 1994) entwickelten Fragebogen zur "Controllability of Motor Imagery" (CMIT) und ließ 54 Probanden (darunter Experten und Novizen) sich zum einen Bewegungen in der Erste-PersonPerspektive vorstellen, zum anderen solten sie eines von 5 Bildern der letzten Phase einer Bewegung auswählen und die Bildreihe ergänzen. Er fand heraus, dass die Vorstellung von Bewegung in der internen Perspektive (eigene Bewegung) mit sportlicher Erfahrung korreliert. Je größer die Erfahrung war, desto besser war auch die Vorstellung. Die beobachtungszentrierte Vorstellung (Bewegungen anderer), gemessen mit dem Bilderselektionstest, hing nicht mit der Expertise zusammen. Die Zeit für die Mentale Rotation ist bei Experten kürzer als bei Novizen. Die Ergebnisse werden als Hinweise für zwei verschiedene Arten von motorischer Vorstellungen gewertet: eine internale und eine externale Vorstellung. Korrelationsstudien können allerdings keine Aussagen zu Kausalzusammenhängen machen.

Hierzu gibt es eine Studie, die die Hauptaussage zum Verhältnis zwischen Expertise und Vorstellungsart repliziert: Frester (1996) befragte Nachwuchstalente in 4 Disziplinen (Wasserspringen, Hoch- und Dreisprung sowie Kanu-Slalom) nach den Bedingungen, die den Aneignungsprozess der Mentalen Repräsentation (Bewegungswissen) fördern. Er fand drei Hauptergebnisse: Erstens werden hauptsächlich bildhaft-anschauliche Repräsentation an den Knotenpunkten der Bewegung gebildet. Zudem sind hierzu 
metaphorische Hilfen förderlich. Zweitens ist ein flüssiges Verbinden der Bewegungsphasen wichtig und drittens sind bildhaft-anschauliche und kinästhetische Vorstellungen verwoben. Kritisch wird hier die Befragungsmethode gesehen. Zwar ergänzt Frester (ebd.) die Untersuchung mit einem Training der Bewegungsausführung, doch müssen oder können nicht alle Details ins Bewusstsein gelangen, d.h. sie sind nicht bewusstseinspflichtig oder sind nicht bewusstseinsfähig. Es ist nicht auszuschließen, dass vorherige Annahmen der Forscher beim Interpretationsprozess der Interviews in die Auswertung hineinkommen. Beide Studien haben demnach Nachteile, die erstere ist eine Korrelationsstudie und die zweite eine Befragung (vergleiche auch Hinweise im Kapitel über die Methoden zur Untersuchung von Vorstellungen und deren Kritik. Siehe dazu auch Kap. 5.3).

De Beni und Pazzaglia (1978, zit. nach Kosslyn, Behrmann \& Jeannerod, 1995) fanden heraus, dass autobiografische Vorstellungen (im Vergleich zu anderen Kontexten) die meiste Zeit beanspruchten, um generiert zu werden, aber zu einem sehr guten Abruf führten und sehr lebendig waren.

Hinweise auf eine diskontinuierliche Entwicklung gibt es bei der folgenden Studie: Reed (2002, zit. nach Moran, 2004) untersuchte drei Gruppen mit unterschiedlichem Expertenlevel (hoch, mittel, gering) beim Springen von einem Sprungbrett mit dem Mentalen Chronometrie-Paradigma. Ziel war es zu prüfen, ob die zeitliche Relation zwischen Vorstellung und Bewegungsausführung Unterschiede des Inhalts und der Struktur der motorischen Repräsentationen aufweist, die mit dem Expertise-Niveau zusammenhängen. Die Gruppen sollten sich den Sprung vorstellen und auch ausführen. Es wurden die jeweiligen Zeiten dafür verglichen. Dabei konnte festgestellt werden, dass die mittlere Expertisegruppe länger für die Vorstellung als für den tatsächlichen Sprung brauchten als die Novizen und Experten. Dies wurde im Sinne von Unterschieden in der Bewegungsrepräsentation interpretiert. Dabei soll, so Reed (ebd.), die mittlere Gruppe mehr vom noch nicht automatisiertem Wissen aktualisieren. Ergänzend waren die Zeiten für den Faktor Komplexitätsgrad bei vorgestelltem und tatsächlichem Sprung vergleichbar und monoton ansteigend, d.h. je komplexer der Sprung, desto länger war die Zeit.

Allerdings muss die Definition des Expertise-Niveaus genauer betrachtet werden. Diese ist von Studie zu Studie verschieden. Möglicherweise hatten die Sportler der mittleren Fertigkeitsgruppe die Prozeduren und Bewegungsregeln noch nicht gefestigt. Auch 
wurde keine Messung der Fähigkeiten durchgeführt. Die Expertise ist eigentlich eine Erfahrungsvariable, sie hat nur indirekt mit dem Beherrschungsgrad der Sportart zu tun. Dabei wird hier in der Dissertation die motorische Fertigkeit durch ein Expertenrating bewertet und geht so mit in die Analyse ein.

Bohan et al. (1999) wollten herausfinden, unter welchen Bedingungen Mentales Training die besten Effekte verursacht. Sie untersuchten drei Gruppen unterschiedlicher Expertise mit einem Vor- und Nachtest. Dieser Effekt des mentalen Trainings lässt mit zunehmender Beherrschung der Fertigkeit nach. Bei Experten ist demnach das Mentale Training weniger effektiv als bei Novizen. Dies kann nach Meinung der Autorin der Dissertation daran liegen, dass Experten schon über automatisierte Strategien verfügen, die sie bereits anwenden. Deshalb sind sie möglicherweise nicht mehr so gut trainierbar. Allerdings weisen Driskell et al. (1994) auch darauf hin, dass der Wissensstand hierzu nicht einheitlich ist.

In der Literatur zum mentalen Training, auf die hier nur in den für die Dissertation notwendigen Fragen eingegangen wird, heißt es in einer der wichtigsten klassischen Studie dazu folgendes: Laut der Metaanalyse von Driskell et al. (1994, zit. nach Moran 2004) ergibt sich eine Interaktion zwischen Niveau der motorischen Bewegungsausführung und dem Aufgabentyp beim mentalen Training: Experten profitieren unabhängig vom Aufgabentyp gleich gut vom mentalen Training. Novizen hätten hingegen bei kognitiven Aufgaben vom mentalen Üben einen größeren Nutzen. Bei Metaanalysen gelten die von Bortz und Döring (2006) genannten kritischen Hinweise.

\subsubsection{Fazit}

Vorstellungen aus der Beobachterperspektive (oder beobachtungsbezogene motorische Vorstellungen) sollen für das Lernen von Bewegungen insoweit förderlich sein, als dass sie den exakten Erwerb durch den genauen inneren Nachvollzug der Bewegung fördern. Sie sollen weniger erfahrungsabhängig als körperzentrierte (oder eigenmotorische) Vorstellungen sein. Umgekehrt sollen Vorstellungen aus der Erste-Person-Perspektive abhängig von Erfahrungen mit Bewegungen sein. Autobiografische Vorstellungen seien zeitaufwendiger als Vorstellungen, die in anderen Kontexten erzeugt werden. Es lassen sich Diskontinuitäten in der Entwicklung von Vorstellungsfähigkeiten aufzeigen, was auf unterschiedliche Automatisierung des Bewegungswissens bezogen wird. 


\subsection{Vorstellung und Geschlecht}

Im Folgenden werden psychologische Befunde zu der Variablen Geschlecht im Zusammenhang zu Vorstellungen referiert.

Es werden verschiedene Faktoren angenommen, die die Geschlechterunterschiede erklären können. Sie werden beispielhaft anhand einzelner Studien erläutert.

\subsubsection{Mögliche Ursachen für Geschlechterunterschiede}

\subsubsection{Aufgabenart}

Werden die zugrundeliegenden Faktoren von Fragebögen zur Messung von Vorstellungsfähigkeiten analysiert, dann zeigen sich zwei unabhängige Faktoren: die verbale Fähigkeit und die visuell-räumliche Fähigkeit (Isaac \& Marks, 1994). Dabei besteht die visuell-räumliche Fähigkeit wiederum aus zwei Komponenten (McGee, 1979, zit. nach Halpern, 1986):

- Einen Visualisierungsfaktor; der etwa die Fähigkeit beinhaltet, sich vorzustellen, wie Objekte aussehen, wenn sie rotiert werden, zu imaginieren, wie ein flaches Objekt erscheint, wenn es gefaltet wird oder wie ein Körper aussieht, wenn er auseinandergefaltet wird.

- Der zweite ist der Orientierungsfaktor. Er beinhaltet die Fähigkeit, Beziehungen zwischen verschiedenen Reizen zu erkennen und die räumlichen Muster zu erfassen.

Es wird angenommen, dass Frauen eine variablere Leistung beim Faktor Visualisierung und Männer größere Variabilität beim Faktor Orientierung aufweisen (Halpern, 1986).

Demnach bestimmt die Art der Aufgabe, ob es Geschlechterunterschiede gibt.

Kimura (1999) fasst dies noch einmal zusammen:

Männer seien Frauen bei der Mentalen Rotation überlegen (Collins \& Kimura, 1997, zit. nach Kimura, 1999), während Frauen die Positionen von Objekten auf einem Feld besser aus dem Gedächtnis abrufen könnten (etwa, wenn im Experiment eines von einander zugehörigen Objektpaaren vertauscht wird) (Eals \& Silverman, 1994, zit. nach Kimura, 1999).

Es wird angenommen, dass es sich hierbei um zwei sehr verschiedene Fähigkeiten handelt, denn je nach Test gebe es sogar mehr oder weniger große Geschlechterunterschiede (Kimura, 1999): 
- Größere Unterschiede gebe es bei den Aufgaben, welche die räumliche Orientierung beinhalten (sie beinhalten die Korrektur von Orientierungsänderungen, ohne eine Manipulation der Teile des Objekts), beim Zielen und beim räumlichen Ortsgedächtnis (wobei hier Frauen teilweise Männern überlegen sein können).

- Kleinere Geschlechterunterschiede gebe es bei folgenden Aufgabenbereichen: räumliche Visualisierung (siehe Faktor Orientierung oben), „disembedding“ (dem Herauslösen von Objektteilen aus einem Gesamtobjekt) und räumliche Wahrnehmung (mit dem Merkmal Feldunabhängigkeit). Letzteres entspricht der Fähigkeit, eine räumliche Orientierung in einem bestimmten Umfeld zu identifizieren, zum Beispiel die Orientierung einer Senkrechten in einem gekippten Rahmen zu bestimmen, während die Person selbst auf einem gekippten Stuhl sitzt, ohne Bodenkontakt zu haben.

Vecchi und Girelli (1998) stellten fest, dass Männer eher Vorteile bei Aufgaben mit dynamischen Anteilen haben und Frauen eher bei Aufgaben mit passiven Merkanteilen. Sie referieren die Studien von Linn und Petersen (1985), die Vorteile bei männlichen Probanden bei der räumlichen Wahrnehmung und der Mentalen Rotation sehen und dagegen weibliche Studienteilnehmer bei räumlichen Visualisierungsaufgaben besser abschneiden. Auch Voyer et al. (1995) fanden in einer Metaanalyse heraus, dass bei Aufgaben mit dynamischen Aspekten - etwa der Mentalen Rotation und der Transformation mentaler Bilder - männliche Teilnehmer besser abschnitten als weibliche. Bei Kindern wurde dieser Einfluss nach dem Wissenstand der Autorin dieser Dissertation nur selten untersucht. Dies war z.B. bei Kosslyn et al. (1990) der Fall (siehe Kap. 7.5.4).

\subsubsection{Strategien}

Nicht nur die Aufgabenart beeinflusst die Unterschiede zwischen Frauen und Männern. Auch die Art der Strategie ist ein wichtiger Faktor, denn geschlechtsspezifische Unterschiede (wie übrigens auch Altersunterschiede) werden durch die Verwendung von verbalen Strategien vermindert (Clarkson-Smith \& Halpern, 1983, zit. nach Halpern, 1986).

Andere Untersuchungen zur unterschiedlichen Verwendung von Strategien bei Mentalen Rotationen wurden von Heil und Jansen-Osmann (2008a) durchgeführt. Dabei wurde der Einfluss zweier Faktoren (Komplexitätsgrad und Geschlecht) hinsichtlich der Reaktions- 
geschwindigkeit beim Mentalen Rotieren von Polygonen ins Visier genommen. Als Ergebnis stellten die Autoren heraus, dass Männer besser als Frauen abschnitten. Je komplexer die Polygone, desto schlechter war die Reaktionszeit. Dieser Effekt verstärkte sich noch bei Frauen und komplexen Polygonen, während er bei Männern gleich blieb. Die Autoren interpretieren dies mit einer holistischen Strategie bei Männern und einer analytischen Strategie bei Frauen: „If men prefer a holistic mental rotation mode, then their mental rotation speed should be faster than women's and should be independent of the polygons' complexity. If women prefer an analytic mental rotation mode, however, then their mental rotation speed should be slower for more complex than for simple polygons“ (Heil \& Jansen-Osmann, 2008a, S. 687). Ein unterstützender Befund kommt von Jordan et al. (2002) hinzu. Sie untersuchten mittels fMRT-Studie solche Probanden, die in drei verschiedenen Mentalen Rotationstests keine Geschlechterunterschiede zeigten. Sie fanden jedoch Geschlechterunterschiede bei der Aktivierung des Kortex, die ebenfalls auf die Wahl geschlechtsspezifischer Strategien zurückgeführt werden: Frauen sollen hierbei analytisch, Männer holistisch vorgehen.

Vecchi und Girelli (1998) verglichen Geschlechterunterschiede bei zwei ähnlichen Aufgaben, eine stellte eher Anforderungen dynamischer Art und die andere verlangte eher die Nutzung passiven Merkens. Dabei sollte einem zuvor gelernten Pfad mental gefolgt werden bzw. der Ort von in einer Matrix ausgefüllter Kästchen gemerkt und danach vorgestellt werden. Männer waren bei der ersten Aufgabe besser, Frauen hingegen bei der zweiten Aufgabe. Dies interpretierten die Autoren dahingehend, dass die visuell-räumlichen Fähigkeiten ein komplexes Konstrukt sind. Das bedeutet, dass unterschiedliche Fähigkeiten dazu beitragen. Inwieweit hier allerdings der Faktor „Strategienutzung“ kontrolliert wurde, ist unklar, denn bei beiden Bedingungen kann eine verbale Strategie genutzt werden, um sich den Pfad und um sich den Ort des ausgefüllten Kästchens zu merken. Je nachdem, ob bei der Mentalen Rotation eine analytische oder eine holistische Strategie angewendet wird, ist die zusätzliche Verwendung von verbalen Strategien eher bei der analytischen Strategie erdenklich. Ein zusätzliches Experiment, bei dem keine verbale Strategie möglich ist, ist anzuraten.

\subsubsection{Expertise}

Ein Einflussfaktor könnte neben den Strategien die Expertise (im Sinne von Übung) sein. Isaac und Marks (1994) nutzten einen Selbstbeobachtungsfragebogen, um entwicklungsbezogene Änderungen und Geschlechterunterschiede bei Kindern im Alter von 7-8, 
8-9 und 10-11 Jahren und Erwachsenen bis zu 50 Jahren (bei insgesamt 547 Probanden) zu erforschen. Die Messinstrumente waren die Fragebögen „Vividness of Visual Imagery Questionnaire“ und „Vividness of Movement Imagery Questionnaire“. Es wurden bedeutsame Veränderungen bei Kindern ab 8 Jahren gefunden. Die Mädchen berichteten über eine lebendigere Vorstellungskraft als Jungen. Die Kinder mit geringen motorischen Fertigkeiten waren auch schlecht bei den Vorstellungen (hiervon berichteten 42 Kinder keine Vorstellungsbilder). Die jungen Erwachsenen mit Erfahrung in einer oder mehr Sportarten waren auch beim Bilden von Vorstellungen denen ohne Erfahrung überlegen. Diese Ergebnisse sprechen laut den Autoren für die bedeutende Rolle von Vorstellungen bei der Handlungsplanung. Hierbei handelt es sich allerdings um eine korrelative Studie, die Aussagen zur Kausalität nicht ermöglicht. Es gilt auch die Kritik aus Kapitel 5.3.1.

Eine Studie von Neubauer, Bergner und Schatz (2010) untersuchte Trainingseffekte bei der Mentalen Rotation von den Figuren wie sie Shepard und Metzler (1971) verwendeten und eine Version in 3D, wie sie in einem Computerprogramm in der "Virtuellen Realität“ (VR) geschaffen wurde. Dabei zeigten sich im Elektroenzephalogramm (EEG) Trainingseffekte für beide Varianten der mental zu drehenden Objekte, denn die Gehirnaktivität verminderte sich hierbei. Geschlechtereffekte bei den 15-jährigen Jugendlichen konnten für die Figuren in der VR nicht nachgewiesen werden, doch die Geschlechterunterschiede für die 2D-Figuren verschwanden nach dem Training. Genauer: Bei männlichen Jugendlichen konnte weniger Gehirnaktivität im EEG nach dem Training für die beiden Bedingungen gemessen werden, bei weiblichen Jugendlichen verbesserte das Training nur die Rotationsfähigkeit der 3D-Figuren.

Es lassen sich laut Kimura (1999) aber durch das Üben nur Kurzzeiteffekte nachweisen. Sinnvoll sind also „follow-up-Studien“, die einen größeren Zeitraum nach dem Training einschließen, so dass diesem Argument begegnet werden kann. Zusammengefasst bedeutet das Ergebnis einen Einfluss der Expertise auf die Leistungen der Mentalen Rotation: Während beim Experiment von Neubauer et al. (2010) männliche Jugendliche mit mehr Training auch bessere Leistungen bei der Mentalen Rotation von 3D-Objekten in der Virtuellen Realität erzielten, blieben die Leistungen der weiblichen Jugendlichen konstant. Ohne Training konnten hierbei keine Unterschiede der Geschlechter gefunden werden. Womöglich haben Männer mit Art und Umgang der bzw. mit den 2D-Figuren mehr Erfahrung als Frauen, denn bei relativ ungewohntem Reizmaterial verschwinden diese Geschlechterunterschiede. In der oben berichteten Studie haben beide 
Geschlechter keine oder wenig visuelle Erfahrung mit den Objekten, was für die Autorin dieser Dissertation hierbei der ausschlaggebende Faktor sein könnte.

Bei Rilea (2008) wurden 67 erwachsenen Rechtshändern Aufgaben zur Mentalen Rotation mit menschlichen Figuren, Buchstaben und 2D-Objekten gezeigt. Während keine Geschlechterunterschiede bei der Mentalen Rotation von menschlichen Figuren gefunden wurden, bestanden diese bei Buchstabenrotationen. Dabei zeigte sich nur bei Frauen eine stärkere Lateralisierung in der linken Hemisphäre. Dies wurde von den Autoren im Sinne der Verwendung einer verbalen Strategie gewertet. In der Kontrollbedingung (2D-Objektrotation) wurden unerwartet keine Unterschiede zwischen den Geschlechtern gefunden.

Demnach ist das Reizmaterial und der Bekanntheitsgrad mit diesem Material bestimmend, ob es Unterschiede zwischen Männern und Frauen gibt. Somit könnte die Expertise eine Moderatorvariable für das Geschlecht sein.

\subsubsection{Lateralisierung und andere Entwicklungsfaktoren}

Eine weitere Erklärung für die Geschlechterunterschiede ist die unterschiedliche Hemisphärenlateralisation bei Jungen und Mädchen ab dem Alter von 5 Jahren: Hahn, Jansen und Heil (2009) untersuchten mittels EEG die elektrophysiologischen Korrelate im Gehirn. Hierbei fanden sie Hinweise auf bilaterale Aktivitäten bei Jungen und linkslaterale Aktivitäten bei Mädchen, während sie Objekte mental rotierten. Die Autoren schlussfolgerten, dass noch vor der hormonellen Umstellung in der Pubertät die unterschiedliche Entwicklung und Funktion des Gehirns eine Rolle spielt.

In den folgenden Kapiteln 7.7.2 und 7.7.3 dargestellten Studien von Karádi et al. (1999) sowie Hoyek et al. (2009) sahen die Autoren ihre Ergebnisse im Zusammenhang von entwicklungsbedingten Veränderungen während der späteren Kindheit und Adoleszenz.

\subsubsection{Einfluss der Instruktion und stereotype Erwartungen}

Sharps, Price und Williams (1994) zeigten, dass die Instruktion und die spezifischen Kontextinformationen, in denen die Aufgaben eingebunden sind, die Ergebnisse so beeinflussen, dass bei Kontrolle dieser Variablen keine Geschlechterunterschiede mehr sichtbar werden. So etwa wurden bei der Bedingung mit Instruktion die räumlichen Merkmale bei der Mentalen Rotation betont (bzw. nicht betont). In der Kontextbedingung wurde auf die Nutzung von räumlichen Fähigkeiten bei bestimmten geschlechtstypischen Berufen hingewiesen. In der Instruktionsbedingung konnten keine Unterschiede ge- 
funden werden, wenn keine Hinweise auf die räumlichen Merkmale gegeben wurden. Bei der Kontextvariation ergab sich eine Interaktion zwischen Geschlecht und Kontextinformation. Hierzu führen die Autoren als Erklärung mögliche soziokulturelle Erwartungen an das jeweilige Geschlecht an.

\subsubsection{Mentalen Rotation und Geschlechterunterschiede bei Kindern}

Es sind der Autorin dieser Dissertation nur sehr wenige Untersuchungen bekannt, die bei Kindern im Alter zwischen 4 und 10 Jahren Geschlechterunterschiede und deren Ausprägung beim Mentalen Rotieren oder mit anderen Verfahren untersuchen.

Wenn es um Befunde zur Mentalen Rotation geht, werden darunter Fähigkeiten verstanden, mehrdimensionale Objekte in der Vorstellung zu drehen (Linn \& Petersen, 1985).

Während weibliche Erwachsene im Durchschnitt länger brauchen als männliche Erwachsene, wenn sie mental rotieren sollen (Hirsch et al., 2003; Roberts \& Bell, 2003; Shepard \& Metzler, 1971), sind die Befunde bei Kindern rar und kontrovers. Einige wenige werden hier erläutert:

Karádi et al. (1999) untersuchten eine Gruppe Kinder ( $N=89)$ im Alter von 9 Jahren und eine Kontrollgruppe von 48 jungen Erwachsenen mit einer Mentalen Rotationsaufgabe hinsichtlich der Geschlechterunterschiede. Dabei sollten Hände in der Vorstellung rotiert werden (Rotation in der Erste-Person-Perspektive). Es zeigte sich, dass Unterschiede zwischen den Geschlechtern erst ab einem späteren Alter (>9 Jahren) bedeutsam waren.

Geschlechterunterschiede fanden Heil und Osmann (2008b) bei 8-jährigen Kindern hinsichtlich der Genauigkeit, mit der die Mentale Rotation durchgeführt wurde, nicht aber bei der Geschwindigkeit. Die Mentale Rotation von 109 Mädchen und Jungen zwischen 7 und 8 Jahren wurde hierbei mittels psychometrischer und chronometrischer Methoden untersucht.

Hahn (2010) hat in ihrer Dissertation bei 4- bis 6-jährigen Kindern untersucht, ob sich bei der Mentalen Rotation von bunten Figuren (z.B. ein Krokodil) oder von Buchstaben Geschlechterunterschiede zeigen. So erhielt sie bei bunten Figuren als Ergebnis, dass sich Mädchen und Jungen zwar nicht bezüglich der Reaktionszeiten unterschieden, die Jungen aber hinsichtlich der Fehlerrate eine bessere Leistung zeigten, wenn dabei Mentale Rotation erforderlich war. Beim Reizmaterial Buchstaben unterschieden sich 
Mädchen und Jungen weder bezüglich der Fehler noch der Reaktionszeit.

Die fehlenden Geschlechterunterschiede bei dem Buchstabenmaterial könnten darauf zurückzuführen sein, dass sowohl Jungen als auch Mädchen mit Buchstaben weniger Erfahrung haben als mit bunten Figuren. Jungen hätten demnach anscheinend mehr Erfahrung mit bunten Figuren als Mädchen.

Levine, Huttenlocher, Taylor und Langrock (1999) untersuchten mittels einer neuen Aufgabe bei Kindern, die im Mittel 4;6 Jahre alt waren, ob es Geschlechterunterschiede eher beim Verschieben oder eher beim Rotieren gibt. Dazu entwickelten sie eine Aufgabe, bei der das Verschieben bzw. Rotieren von geteilten 2D-Figuren zur vollständigen Figur notwendig war. Jungen waren den Mädchen ab dem Alter von 4;6 Jahren überlegen. Als Ergebnis erhielten die Autoren zudem Hinweise darauf, dass Jungen in diesem Alter eher beim Verschieben einen Vorteil hatten, nicht beim Rotieren. Ein Vorteil intellektueller Entwicklung der Jungen konnte ausgeschlossen werden. Kritisch ist zu bewerten, dass die 4 Aufgabentypen nicht streng genug nach Verschiebeund Rotationsaufgabe getrennt werden können.

Voyer, Voyer und Bryden (1995) untersuchten in ihrer Metaanalyse ebenfalls die Größe der Geschlechterunterschiede bei räumlichen Fähigkeiten u.a. auch bei der Mentalen Rotation. Dabei wird in ihrer Auflistung der Studien deutlich, dass bei zwei der fünf Studien, die sich mit der Altersspanne von 4 bis 10 Jahren befassen, Vorteile zugunsten der Jungen gefunden wurden. Bei drei Studien sind keine Geschlechterunterschiede erkennbar. Aus den Angaben bezüglich der Effektstärken der zusammengefassten Studien zur Mentalen Rotation wird auch deutlich, dass die Effektstärken mit dem Alter zunehmen ( 0.33 unter 13 Jahren, 0.45 zwischen 13 und 18 Jahren, 0.66 über 18 Jahre). Dies deutet darauf hin, dass der Test der Mentalen Rotation hinsichtlich des Geschlechts in jungen Jahren nicht so gut differenzieren kann (siehe auch Fazit in Kap. 7.7.4). Die Effektstärke ist ein Maß für einen praktisch bedeutsamen Unterschied (Bortz, 1993).

\subsubsection{Befunde sonstiger Methoden bei Kindern}

Mit einem Mentalen Chronometrie-Paradigma untersuchten die Forscher Hoyek et al. (2009) Kinder ( $\mathrm{N}=30$ ) im Alter zwischen 7 und 8 Jahren und Schulkinder im Alter von 11 und 12 Jahren $(\mathrm{N}=61)$. Anhand eines Hindernisparcours, der mental und aktiv durchlaufen werden sollte, wurden die Zeiten für diese beiden Aufgabentypen verglichen. Es ergaben sich stärkere Zusammenhänge zwischen mentaler und tatsächlich 
gebrauchter Zeit bei den älteren Kindern. Der Zusammenhang war bei Jungen eher zu finden als bei Mädchen.

Waber, Carlson und Mann (1982) untersuchten Kinder der Klassen 5 im Alter von 10;9 und 11;0 Jahren und der Klassenstufe 7 im Alter von 12;9 Jahren bei einer tachistoskopischen Präsentation von Buchstaben, die in verschiedenen Winkeln rotiert wurden. Aufgabe war zu entscheiden, ob diese richtig oder seitenverkehrt stehen. In einer Bedingung wurden Hinweise zur Identität und Orientierung des Reizes, in der anderen keine zusätzliche Information gegeben. Kinder können mit zunehmendem Alter besser mental rotieren und Zusatzinformationen nutzen. Es wurden hier keine Geschlechterunterschiede gefunden. Es gab allerdings sozioökonomische Einflüsse, diese aber eher bei den jüngeren Kindern. Eine chronometrische Analyse ergab, dass Kinder mit besserer Rotationsfähigkeit auch genauer in der zeitlichen Übereinstimmung von vorgestellter und physischer Rotation waren.

\subsubsection{Fazit}

Aus der Gesamtheit der Studien hinsichtlich der Betrachtung des Geschlechts folgt: es gibt noch keine Klarheit über Geschlechterdifferenzen und die Größe eventueller Unterschiede. Es besteht der Verdacht, dass der Test der Mentalen Rotation nicht ausreichend hinsichtlich des Geschlechtes differenzieren kann. Ein Vergleich von Tests zur Mentalen Rotation mit Tests zur Mentalen Verschiebung (Levine et al., 1999) weisen auch darauf hin, haben aber, wie oben angesprochen, methodische Mängel.

Die Befunde zu Geschlechterunterschieden, gerade wenn es um das Kindesalter geht, sind nicht immer übereinstimmend mit der Befundlage bei Erwachsenen und teilweise widersprüchlich. Die Ergebnisse der Studien deuten darauf hin, dass zum einen das Vorwissen bzw. die Expertise eine mögliche moderierende Variable darstellt. Hierbei wurde auf die visuelle Erfahrung mit dem Reizmaterial und der alleinigen Trainierbarkeit von 3D-Firguren bei Jungen (nicht so bei Mädchen) hingewiesen. Auch wird von einigen Autoren angenommen, dass Strategien einen Einfluss auf Geschlechterunterschiede haben. Andererseits lassen sich Geschlechterunterschiede nicht mehr nachweisen, wenn Kontextinformationen kontrolliert werden, die Geschlechtsstereotype in den Reaktionen hervorrufen. Als Erklärung wird auch die geschlechtsspezifische Lateralisierung herangezogen.

Im Test für die Mentale Rotation sind Geschlechterunterschiede schon vor dem Alter von 
13 Jahren erkennbar. Der Beginn der Altersgrenze hierfür hängt jedoch vom jeweiligen Test ab. Zum Beispiel können Geschlechterunterschiede beim Messen der Mentalen Rotationsgeschwindigkeit nachgewiesen werden. Im Gegensatz dazu wurden bei den Genauigkeitsparametern keine solchen Unterschiede gefunden.

Es ergibt sich demnach die Notwendigkeit der begrifflichen Ausdifferenzierung und der Validierung der Tests räumlicher Fähigkeiten. 


\section{Empirische Untersuchungen}

\subsection{Hypothesen}

Es werden im empirischen Teil aus den eingangs gestellten Fragen $(F)$ abgeleitete Hypothesen $(\mathrm{H})$ sowie deren statistische Hypothesen $(\mathrm{SH})$ mit den statistischen Tests (ST) wie folgt untersucht (die $H_{0}$ entspricht der Nullhypothese, die $H_{1}$ der Alternativhypothese):

\section{Zum Zusammenhang motorischer und kognitiver Leistungen in der Kindheit}

(Studie 1)

$\mathrm{F}_{1}$ : Wie hoch ist der Zusammenhang zwischen dem Bewegungslernen und den Wahrnehmungs- und Vorstellungsleistungen?

$\mathrm{H}_{(\mathrm{F} 1)}$ : Es können signifikante Korrelationen zwischen dem Bewegungslernen und den Wahrnehmungs- und Vorstellungsleistungen aufgezeigt werden.

$\mathrm{SH}_{(\mathrm{F} 1)}: \mathrm{H}_{1}: 0<\mathrm{e}<=1 \quad \mathrm{H}_{0}: \mathrm{\varrho}=0$

$S T_{(F 1):}$ Korrelationsrechnung (nach Pearson)

\section{Zum Einfluss von Erfahrung auf Wahrnehmung und Vorstellung von Bewegungen} in der Kindheit (Studie 2)

F2: Welchen Einfluss hat der Faktor Erfahrung auf die Wahrnehmungs- und Vorstellungsleistungen?

$\mathrm{H}_{(\mathrm{F} 2)}$ : Eine größere Erfahrung hat einen positiven Einfluss auf die Wahrnehmung und Vorstellung von Bewegungen in der Kindheit. Das bedeutet, dass die Tenniskinder (TK) gegenüber den untrainierten Kindern (UK) einen Vorteil bei der Lösung der Wahrnehmungs- und Vorstellungsaufgaben haben.

$\mathrm{SH}(\mathrm{F} 2): \mathrm{H}_{1}: \mu_{\mathrm{TK}}>\mu_{\mathrm{UK}} \quad \mathrm{H}_{0}: \mu_{T K}<=\mu_{U K}$

ST(F2): Varianzanalyse; Erfahrung als between-subject-Faktor

\section{Zum Einfluss des Geschlechts auf Wahrnehmung und Vorstellung von Bewegungen in der Kindheit (Studie 3)}

F3: Welchen Einfluss hat der Faktor Geschlecht auf die Wahrnehmungs- und Vorstellungsleistungen?

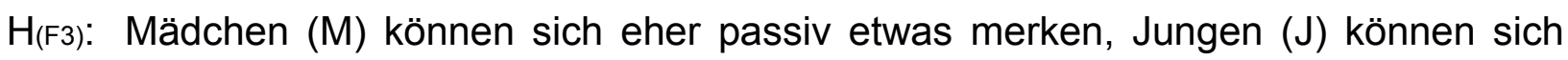


besser etwas dynamisch vorstellen. Jungen $(\mathrm{J})$ haben gegenüber Mädchen $(\mathrm{M})$ einen Vorteil bei der Lösung der Vorstellungsaufgabe. Bei der Wahrnehmungsaufgabe unterscheiden sich die Jungen und Mädchen nicht voneinander.

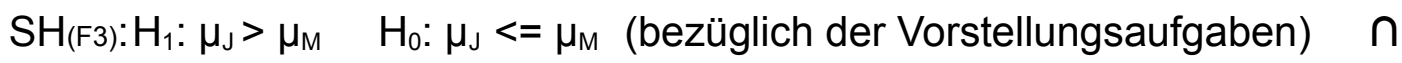

$\mathrm{H}_{1}: \mu_{\mathrm{J}}=\mu_{\mathrm{M}} \quad \mathrm{H}_{0}: \mu_{\mathrm{J}} \neq \mu_{\mathrm{M}} \quad$ (bezüglich der Wahrnehmungsaufgabe)

$\mathrm{ST}_{(\mathrm{F} 3)}$ : Varianzanalyse, Geschlecht als between-subject-Faktor

\section{Zum Einfluss der Versuchsbedingung auf Wahrnehmung und Vorstellung von Bewegungen in der Kindheit (Studie 4)}

F4: Hat die Versuchsbedingung einen Einfluss auf die Wahrnehmung und Vorstellung von Bewegungen in der Kindheit?

$\mathrm{H}_{(\mathrm{F} 4)}$ : Die beiden Vorstellungsbedingungen „50z“ und „50g“ unterscheiden sich von der Wahrnehmungsbedingung „0“.

$\mathrm{SH}_{(\mathrm{F} 4)}: \mathrm{H}_{1}: \mu_{0} \neq \mu_{50 \mathrm{z}} \neq \mu_{50 \mathrm{~g}} \quad \mathrm{H}_{0}: \mu_{0}=\mu_{50 \mathrm{z}}=\mu_{50 \mathrm{~g}}$

ST(F4): Varianzanalyse, Versuchsbedingung als within-subject-Faktor

\section{Zum Einfluss von Trainingserfahrung auf Wahrnehmung und Vorstellung von Bewegungen in der Versuchsgruppe (Studie 5)}

F5: Welchen Einfluss hat der Faktor Trainingserfahrung auf die Wahrnehmungs- und Vorstellungsleistungen in der Versuchsgruppe?

$\mathrm{H}_{(\mathrm{F} 5)}$ : Die älteren Kinder (AK) der Versuchsgruppe haben gegenüber den jüngeren Kindern der Versuchsgruppe (JK) keine Vorteile bei der Wahrnehmung und Vorstellung von Bewegungen.

$\mathrm{SH}_{(\mathrm{F} 5)}: \mathrm{H}_{1}: \mu_{\mathrm{AK}}=\mu_{\mathrm{JK}} \quad \mathrm{H}_{0}: \mu_{\mathrm{AK}} \neq \mu_{\mathrm{JK}}$

$\mathrm{ST}_{(\mathrm{F} 5)}$ : Die Versuchsgruppe wurde anhand des Altersmedians geteilt, was den zwei unterschiedlich trainierten Gruppen der Versuchsgruppe entsprach. Es wurde eine Varianzanalyse mit den beiden unterschiedlich trainierten Gruppen als Gruppenfaktor durchgeführt.

Zum Einfluss von Trainingserfahrung auf Veränderungen im Bewegungslernen in der Versuchsgruppe (Studie 6)

F6: Welchen Einfluss hat die Trainingserfahrung auf Veränderungen im Bewegungs- 
lernen in der Versuchsgruppe? Gibt es Hinweise dafür, dass unterschiedliche Eingangsvoraussetzungen der Kinder dieses Ergebnis mit bedingen?

$H_{(F 6)}$ : Die älteren Kinder der Versuchsgruppe (AK) unterscheiden sich nicht von den jüngeren Kindern (JK) hinsichtlich des Bewegungslernens. Die Eingangsvoraussetzungen sind aufgrund der Stichprobenziehung (Klumpenstichprobe) bei den Kindern nahezu gleich.

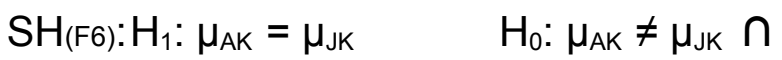

Es wird kein Einfluss der Kovariaten angenommen.

ST(F6): Es wurde eine Kovarianzanalyse mit dem Faktor Trainingserfahrung als festen Faktor berechnet. Die Versuchsgruppe wurde anhand des Altersmedians geteilt, was den zwei unterschiedlich trainierten Gruppen der Versuchsgruppe entsprach. Die Ermittlung der Variablen Bewegungslernen erfolgte durch die Differenzbildung von zwei Messzeitpunkten (MZP2-MZP3) der Gesamtskala. Die Kovariaten waren: die Anzahl der Sportarten, das Verhältnis der Bewegungsaktivitäten, die Trainingstage in \% (bezüglich der Gesamtdauer des Trainings) und die Ergebnisse des Kasten-Bumerang-Laufs.

\section{Zum Einfluss des Geschlechts auf Veränderungen im Bewegungslernen in der Versuchsgruppe (Studie 7)}

F7: Welchen Einfluss hat der Faktor Geschlecht auf Veränderungen im Bewegungslernen in der Versuchsgruppe? Gibt es Hinweise dafür, dass unterschiedliche Eingangsvoraussetzungen der Kinder dieses Ergebnis mit bedingen?

$H_{(F 7)}$ : Die Mädchen der Versuchsgruppe (M) unterscheiden sich hinsichtlich des Bewegungslernens nicht von den Jungen $(\mathrm{J})$.

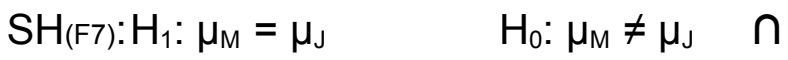

Es wird kein Einfluss der Kovariaten angenommen.

ST(F7): Es wurde eine Kovarianzanalyse mit dem Geschlecht als festen Faktor berechnet. Die Ermittlung der Variablen Bewegungslernen erfolgte durch Differenzbildung von zwei Messzeitpunkten (MZP2-MZP3) der Gesamtskala. Als Kovariaten gingen ein: die Anzahl der Sportarten, das Verhältnis der Bewegungsaktivitäten, die Trainingstage in \% (bezüglich der Gesamtdauer des Trainings) sowie die Ergebnisse des Kasten-Bumerang-Laufs. 


\subsection{Ablauf und Versuchsplanung der Gesamtuntersuchung}

Die Studie nutzt den Ansatz ähnlich dem des Novizen-Experten-Vergleichs. Dabei ist hier die Besonderheit, dass die Novizen untrainierte Kinder sind. Diese Kinder haben keine visuelle oder motorische Erfahrungen mit dem Reizmaterial. Die Versuchsgruppenkinder sind hingegen Lernanfänger, wobei davon auszugehen ist, dass eine visuelle und motorische Erfahrung mit dem Stimulus gegeben ist.

Die Studie wurde in einem quasi-experimentellen Setting durchgeführt, da dies möglicherweise die externe Validität erhöht. Sie wurde begleitend zum Projekt „Jüngsten-Tennis“ in Kooperation mit dem Niedersächsischen Tennisverband in die Praxis umgesetzt.

Nachfolgend wird der Ablauf der Studie exemplarisch verdeutlicht:

- Voruntersuchungen, die zur genaueren Stichprobenbeschreibung dienten (z.B. die Trainingstage). Weiterhin fanden hier die untersuchten Variablen Eingang in die Kovarianzanalyse, um die Eingangsvoraussetzungen der Tenniskinder zu kontrollieren (z.B. die Erfassung der Koordinations- und Antizipationsfähigkeit, welche mit dem Kasten-Bumerang-Lauf gemessen wurde).

- Erfassung des Bewegungslernens: Mittels des „Fragebogens zur Beherrschung der Tennistechnik“" wurden die Videos von Tennisschlägen der Kinder von lizenzierten Tennistrainern analysiert und bewertet.

- Studie zu Wahrnehmungen und Vorstellungen bei der Versuchsgruppe: Durchführung des Versuchs mit mehreren Variationen der abgedeckten Videofläche, dabei wurden folgende Varianten zuerst und in dieser Reihenfolge durchgeführt: Basisbedingung „0“, „50z“ (50\% abgedeckte Fläche, während diese Fläche zusammenhängend ist), „50g“ (50\% abgedeckte Fläche, während diese Fläche nicht zusammenhängend ist), in zufälliger Reihenfolge wurden die Versuche „25“, „33z“, „33g“, 67z“ und „67g“ angeschlossen

- Studie zu Vorstellungen bei der Kontrollgruppe: Durchführung mit drei Varianten abgedeckter Videofläche („0“, „50z“ und „50g“)

- Auswertungsphase

In der folgenden Tabelle 2 wird der Ablauf dargestellt: 


\begin{tabular}{|c|c|c|c|c|c|}
\hline \multicolumn{6}{|c|}{ Studienablauf } \\
\hline & Juni 2005 & Sept. 2005 & $\begin{array}{l}\text { Sommersemester } \\
2006\end{array}$ & $\begin{array}{l}\text { Juni 2006- } \\
\text { April } 2007\end{array}$ & $\begin{array}{l}\text { Mai - Juni } \\
2007\end{array}$ \\
\hline VG & $\begin{array}{l}\text { Kasten- } \\
\text { Bumerang- } \\
\text { Lauf }\end{array}$ & $\begin{array}{l}\text { Bewegungs- } \\
\text { tagebuch }\end{array}$ & $\begin{array}{l}\text { Entwicklung eines } \\
\text { Fragebogens zur } \\
\text { Tennistechnik, } \\
\text { Expertenschulung, } \\
\text { Expertenrating }\end{array}$ & $\begin{array}{l}\text { Versuche: } \\
\text { Basisbed. 0, } \\
\text { 25, 33z/33g, } \\
50 z / 50 \mathrm{~g}, \\
67 \mathrm{z} / 67 \mathrm{~g}\end{array}$ & \\
\hline$K G$ & & & & & $\begin{array}{l}\text { Mündliche } \\
\text { Befragung, } \\
\text { Versuche: } \\
0,50 \mathrm{z}, 50 \mathrm{~g}\end{array}$ \\
\hline
\end{tabular}

Tabelle 2: Der Ablauf der Studie (Legende: Basisbedingung 0, d.h. ohne Flächenabdeckung, 25= 25\% Flächenabdeckung, $\quad 33 z=33 \%$ zusammenhängende Flächenabdeckung, $33 g=33 \%$ getrennte Flächenabdeckung, 50z/50g=50\% zusammenhängende/getrennte Flächenabdeckung) 


\subsection{Studie 1: Zum Zusammenhang motorischer und kognitiver Leistungen in der Kindheit}

Es soll hier der Zusammenhang von motorischen und kognitiven Leistungen am Beispiel des Zusammenhangs von Bewegungslernen und Wahrnehmungs- bzw. Vorstellungsleistungen überprüft werden. Der Hypothese nach sollen Zusammenhänge nachgewiesen werden können. Dies entspricht einer möglichen Validierung des Verfahrens zur Messung von Vorstellungen.

\subsubsection{Stichprobe}

Es wurden zum einen 30 Kinder des Projekts "Jüngsten-Tennis“ untersucht, ein sportliches Angebot für Kinder ab 4 Jahren mit dem Schwerpunkt des Erlernens der Tennistechnik. Es wurde darauf geachtet, dass in die Stichprobe der Versuchsgruppe (VG) auch solche Kinder aufgenommen wurden, die sonst üblicherweise keinen Tennissport treiben konnten. Dies wurde mittels geringer finanzieller Beiträge und Ansprechen von Eltern aus weniger tenniserfahrenen Elternhäusern umgesetzt. Da die Kinder der Versuchsgruppe am Tennistraining teilnahmen, war die Anzahl der Sportarten schon deshalb größer als bei den Kindern der Kontrollgruppe. Im Durchschnitt haben die Kinder der Versuchsgruppe eine Lernerfahrung von ca. 21 Trainingstagen (aufgeteilt auf die 21 Samstage, an denen das Tennistraining stattfand).

Ein besonderer Sachverhalt stellt die unterschiedliche Zusammensetzung der Stichprobe zu unterschiedlichen Zeiten der Untersuchung dar. Während keines der Kinder während der Hauptuntersuchung (Experimente 0,50z, 50g) das Projekt verließ, gab es Wechsel in den anderen Phasen des Projekts. Meist schieden Kinder altersbedingt aus. Es folgten innen jüngere Kinder. Es gab auch ein Kind aus der Versuchsgruppe, das sich der Untersuchung entzog.

In der folgenden Tabelle 3 wird das Alter nach Gruppen angegeben: 


\begin{tabular}{|l|c|c|c|c|c|c|}
\hline \multicolumn{7}{|c|}{ Alter in VG- deskriptive Statistik } \\
\hline & N & Min & Max & Mittelwert & $\begin{array}{c}\text { Standard- } \\
\text { abweichung }\end{array}$ & Varianz \\
\hline ALTER0 & 30 & 5,5 & 10,1 & 8,06 & 1,32 & 1,74 \\
\hline ALTER25 & 18 & 5,5 & 10,6 & 8,28 & 1,52 & 2,31 \\
\hline ALTER33Z & 24 & 5,3 & 10,5 & 8,30 & 1,40 & 1,95 \\
\hline ALTER33G & 23 & 5,4 & 10,4 & 8,46 & 1,48 & 2,18 \\
\hline ALTER50Z & 29 & 5,5 & 10,2 & 8,14 & 1,33 & 1,76 \\
\hline ALTER50G & 27 & 5,5 & 10,5 & 8,37 & 1,38 & 1,89 \\
\hline ALTER67Z & 19 & 5,7 & 10,7 & 7,83 & 1,34 & 1,80 \\
\hline ALTER67G & 22 & 5,5 & 10,2 & 8,00 & 1,25 & 1,57 \\
\hline
\end{tabular}

Tabelle 3: Alter in der Versuchsgruppe (Anmerkung: Alter0=Alter in der Wahrnehmungsbedingung „0“)

Das Geschlechterverhältnis in der Versuchsgruppe wird wie folgt beschrieben (siehe Abb. 6):

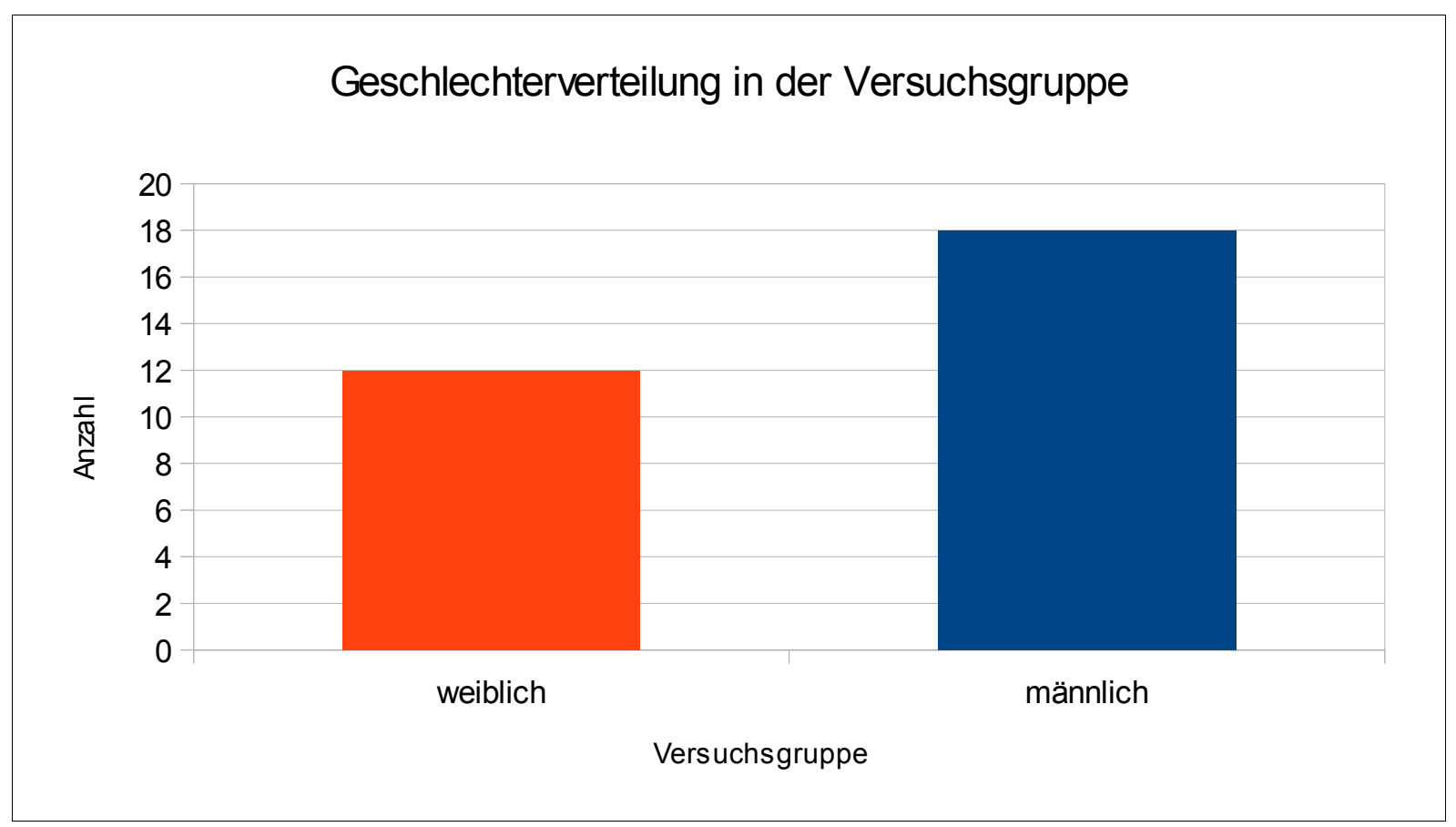

Abbildung 6: Geschlechterverteilung in der Versuchsgruppe

\subsubsection{Methode}

Um den Zusammenhang von motorischen und kognitiven Leistungen in der Kindheit zu messen, wurden folgende Variablen untersucht:

- motorische Leistungen: das Bewegungslernen

- kognitive Leistungen: die Wahrnehmungs- und Vorstellungsleistung 


\subsubsection{Messinstrumente}

Im Folgenden werden die Instrumente dargestellt, mit denen die zuvor beschriebenen Variablen gemessen wurden.

Das Bewegungslernen wurde mit Hilfe eines Fragebogens ermittelt (siehe Anhang 9.1). Dieser Fragebogen erfasst bedeutende Merkmale der Beherrschung der Tennistechnik. Inhalte des Fragebogens sind das rechtzeitige Einnehmen der Schlagposition (einfach gemessen), die Ausholbewegung (zweifach), die Schlagbewegung (siebenfach), die Ausschwungbewegung (einfach) und die Bewegungskonstanz (einfach). Der Fragebogen verwendet eine vierstufige Ratingskala.

Desweiteren wird dargestellt, wie das Material für die Untersuchung der Wahrnehmungsund Vorstellungsleistung hergestellt wurde.

Es wurden zunächst Videosequenzen eines Tennis spielenden Kindes hergestellt. Das Kind war den späteren Untersuchungsteilnehmern unbekannt und wurde in leichter Abweichung von der Frontalperspektive gefilmt. Diese Perspektive entsprach der, von der aus der Trainer mit den Kindern der Versuchsgruppe arbeitete. Dazu wurde die Digitalkamera DCR-TRV950E der Firma "Sony“ verwendet und anschließend die Aufnahmen mit dem Schnittprogramm „Adobe Premiere Pro 1.5“ in Sequenzen von zwei Sekunden Dauer geschnitten.

Die Sequenz zeigte einen Vorhand-Grundschlag. Die Größe/Auflösung der Videos betrug 360x288 Pixel. Das aufgezeichnete Kind trug bei allen Videoaufnahmen die gleiche Kleidung.

Diese Videos wurden dann mittels proprietärer Software mit vorher maschinell hergestellten Grafiken in mehreren Schritten zusammengefügt und in ein "Java“ausführbares Dateiformat konvertiert.

Folgendes Material wurde mit dieser Methode hergestellt: Für die Basisbedingung wurde das Videomaterial ohne flächenabdeckende Grafik benutzt. Für alle weiteren Bedingungen wurden immer Videopaare wie folgt erzeugt: das erste Video eines Paares enthielt eine schwarze Fläche auf der linken (respektive rechten) Seite oder auf der oberen (unteren) Seite.

Der Prozentsatz der schwarz bedeckten Fläche des ersten Videos von einem Paar betrug 25\%, 33\%, 50\% bzw. 67\%. Weiterhin wurden im Falle von 50\% Flächenabdeckung zusammenhängende Flächen hergestellt, d.h. eine Hälfte des Bildes wurde verdeckt („50z“, siehe Abbildung 7). Es wurden auch unzusammenhängende 
Flächen erzeugt (z.B. wurde 25\% Flächenanteil oben links und 25\% unten links verdeckt= „50g“). Dabei wurden bei unzusammenhängenden und zusammenhängenden Flächenverteilungen gleiche Videos in unterschiedlicher, zufälliger Reihenfolge benutzt. Die Zuordnung zu konkreten Flächenabdeckungen (links/rechts oder oben/unten) wurde hierbei zufällig vorgenommen.

Es wurden von 18 Videovorlagen, die für die jeweiligen Subtest verschieden waren, gezeigt. Davon wurden jeweils 6 Videopaare für den Probedurchgang und die restlichen 12 Paare zufällig ausgewählt. Nur Teilversuche, die im Versuch bei gleichem Flächenabdeckungsanteil zusammenhängende Flächen und nicht zusammenhängende Flächen beinhalteten, basierten auf 18 gleichen Videovorlagen.

Die rein mechanische Konstruktion des Versuchs basiert auf dem Grundsatz, dass erst einmal die Methode getestet werden sollte und Ergebnisse nicht vorweg bestimmt werden sollten.

In der Abb. 7 werden das Untersuchungsmaterial und ein beispielhafter Versuchsablauf schematisch dargestellt.

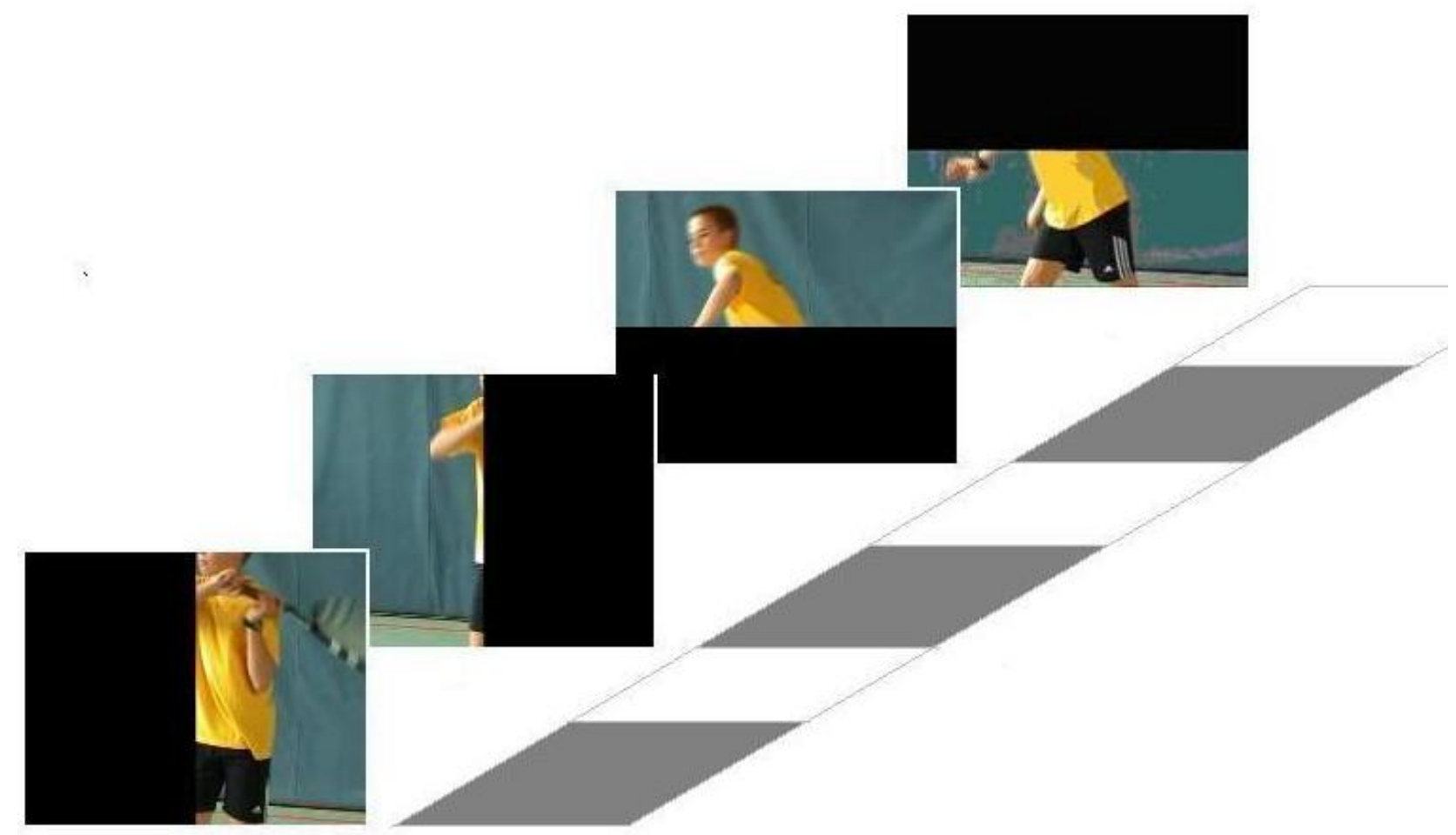

Abbildung 7: Skizze Untersuchungsmaterial und -ablauf (die Trapeze kennzeichnen die Zeitlinie)

Während beim Versuch „50z“ und „50g“ prozentual gleich viel Fläche abgedeckt war, war 
die augenscheinlich sichtbare Fläche beim Versuch „50g“ kleiner als bei „50z“.

\subsubsection{Untersuchungsablauf}

Es folgt der Ablauf für die Messung des Bewegungslernens. Dazu wurde ein Fragebogen (siehe Anhang 9.1) von Studenten entwickelt, die Erfahrung als Tennistrainer aufwiesen. Fünf Rater (lizenzierte Tennistrainer) wurden dann so geschult, dass möglichst eindeutig war, wie eine Bewegung auf dem Fragebogen zu bewerten war.

Die Rater sahen jeweils drei Videosequenzen pro Kind, die zu folgenden drei Messzeitpunkten aufgezeichnet worden waren:

1. MZP: 07.02 .2004

2. MZP: 30.10 .2004 und 13.11.2004

3. MZP:03.09.2005 und 17.09.2005.

Die Experten waren gegenüber den Messzeitpunkten blind.

Es konnte einer der drei Experten nicht in die Auswertung mit einbezogen werden, da ein Fehler bei der Reihenfolge der Kinder auftrat. Zwei der fünf Experten nahmen am späteren Rating aus Zeitmangel nicht teil.

Es wird im Folgenden der Untersuchungsablauf zu den Variablen Wahrnehmung und Vorstellung geschildert.

Während des Projekts „Jüngsten-Tennis“ durchliefen die Tenniskinder (Versuchsgruppe) drei Stationen: ein tennisspezifisches Training, ein Koordinationstraining und eine Station, bei der Spiel und Spaß im Vordergrund standen. Aus diesem Kontext kamen die Kinder aus den unterschiedlichen Situationen zu einem gesonderten Raum, in dem die Untersuchung der Wahrnehmungs- und Vorstellungsleistung stattfand.

Die Untersuchung war freiwillig. Zusätzliche Pausen wurden den Kindern bei Bedarf gewährt. Jedes Kind, das später diese Videos sah, saß in etwa gleichem Abstand vor dem Laptop. Auf eine Zwangshaltung wurde aus ethischen Gründen verzichtet.

Die Videos wurden ohne Ton und ohne Rückmeldung über das Abschneiden in der Mitte des Bildschirms präsentiert.

Die Probanden hatten die Aufgabe per Tastendruck zu entscheiden, ob ein Videopaar gleiche Videos enthielt oder nicht (Wahlentscheidungsaufgabe). Es wurde die „y“-Taste für die Antwort „gleich“ und die „Minus“-Taste für die Antwort „verschieden“ gewählt und mit einer weißen bzw. einer roten Markierung versehen. Die Tasten wurden von den 
Kindern vor dem ersten Versuch probeweise gedrückt, um ein Gefühl für die notwendige Kraft zum Drücken und für das Loslassen zu bekommen und um sicher zu stellen, dass die korrekte Tastenbelegung eingeübt wurde.

Probedurchläufe wurden mit jeweils 6 Videopaaren durchgeführt, um mit dem Versuchsablauf vertraut zu werden.

Jeder Teilversuch bestand aus zwei Abschnitten: Es wurden 6 Videopaare vor einer Pause und 6 Videopaare nach einer Pause gezeigt. Die Pause betrug eine Minute. Der Abstand zwischen den Videopaaren betrug 2 Sekunden wie auch der Abstand zwischen den einzelnen Videos eines Paares. Mit dem Tastendruck wurde die Präsentation des zweiten Videos eines Paares unterbrochen. Es konnte nicht mehr zum ersten Video zurück gesteuert werden.

In der Hälfte der insgesamt 12 Videopaare sind die zwei zugrunde liegenden Videos eines Paares identisch. Kein Videopaar kam doppelt vor.

An drei Tagen in aufeinanderfolgenden Wochen wurde die Untersuchung durchgeführt. Dabei wurden jedem Kind drei Versuche in dieser Reihenfolge Basisbedingung „0“, Wahrnehmungsbedingung „50z“ und „50g“ gezeigt. Die Kinder der Versuchsgruppe nahmen anschließend noch an den Versuchen „25“, „33z“ und „33g“ sowie „67z“ und „67g“ teil. Jedes Kind bekam pro Tag und Woche nur einen Teilversuch zu sehen. Die Basisbedingung ging immer voraus und der Teilversuch mit 50\% zusammenhängend abgedeckter Fläche folgte immer. Anschließend wurde immer der Teilversuch $50 \%$ mit der unzusammenhängend abgedeckten Fläche gezeigt. Die restlichen Teilversuche wurden in zufälliger Reihenfolge präsentiert.

Die Ergebnisse wurden in einer Datei gespeichert, die dann in eine EXCEL-Tabelle und in SPSS überführt wurden. Sie enthielten Informationen darüber, welche Videos mit welcher Abdeckung gezeigt wurden, ob es sich um ein gleiches Videopaar handelte, welche Antwort gegeben wurde und ob die Antwort richtig war. Es folgte die Aufnahme der Reaktionszeit, die hier nur für die Berechnung der Ausreißer benutzt wurde.

\subsubsection{Auswertungsprozedur}

Es wurde zunächst die Interraterreliabilität mittels Korrelationsrechnung bestimmt. Sie stellt die korrelative Übereinstimmung von Raterurteilen dar. Für die Ermittlung des Bewegungslernens wurden die Werte aus dem Fragebogen („Fragebogen zur Beherrschung der Tennistechnik“, siehe Anhang 9.1) über die Beurteilenden gemittelt und eine Gesamtskala (d.h. den Mittelwert über alle Subskalen) gebildet sowie auch die 
Werte aus den Subskalen verwendet. Anschließend wurden jeweils T-Tests für abhängige Stichproben berechnet, um einen möglichen Lernfortschritt beim Bewegungslernen dokumentieren zu können (für die Gesamtskala und für die Subskalen).

Für die Bestimmung der Wahrnehmungs- und Vorstellungsleistung wurden zunächst die Ausreißer anhand der Reaktionszeiten ausgeschlossen. Als Ausreißer wurden Werte mit mehr als 1,5 Quartilsabständen unter und über dem 1. bzw. 3. Quartil aufgefasst (nach Hinweisen von Holling und Schmitz, 2010). Es wurden zudem nur Rechtshänder in die Auswertung eingeschlossen. Weiterhin wurde als abhängige Variable der Prozentsatz richtiger Antworten für jede Untersuchungseinheit (12 Videopaare) pro Kind und für jeden Versuch (z.B. „0“, „50g“ und „50z“) bestimmt.

Es wurde anschließend versucht, Beziehungen zwischen dem Bewegungslernen und den Wahrnehmungs- und Vorstellungsleistungen aufzuzeigen. Hierzu wurden zunächst Korrelationen zwischen dem Bewegungslernen und der Wahrnehmungs- und Vorstellungsleistung berechnet.

Folgende Daten wurden für die Berechnung genutzt: die über alle Beurteiler des „Fragebogens zur Beherrschung der Tennistechnik“ gemittelten Werte (Gesamtskala bzw. Subskalen) wurden mit den Vorstellungs- und Wahrnehmungsleistungen (siehe Kap. 8.3.2.1) korreliert. Die Wahrnehmungsleistung basierte dabei auf den Ergebnissen der Basisbedingung „0“ und die Vorstellungsleistungen basierten auf den Versuchsergebnissen „25“, „33z“ „33g“, „50z“, „50g“, „67z“ und „67g“.

Es musste auf die Darstellung des ersten Messzeitpunktes verzichtet werden, da nicht genügend Kinder, welche zu diesem Zeitpunkt untersucht wurden, später noch im Training verblieben (sogenannte Drop-Outs).

Abschließend wurden Einzelfallbetrachtungen angestellt, eine Rangordnung der Kinder hinsichtlich des Bewegungslernens gebildet (siehe Anhang 9.4) und die Merkmale des Fragebogens als Lernindikatoren betrachtet. Diese werden aus der durchschnittlichen Bewertung einer Subskala der Tennistechnik und deren Rangfolge gebildet.

\subsubsection{Ergebnisse der Studie 1}

Im Folgenden werden die Ergebnisse der Studie 1 dargestellt.

\subsubsection{Interraterreliabilität}

Die Interraterreliabilität ist für die einzelnen Messzeitpunkte in der folgenden Tabelle 4 
abgebildet (siehe auch das Abkürzungsverzeichnis im Kap. 9.5)

\begin{tabular}{|l|l|l|l|}
\hline \multicolumn{4}{|l|}{ Interraterreliabilität } \\
\hline & MZP 1 & MZP 2 & MZP 3 \\
\hline SP & .14 & $.35^{*}$ & $.70^{* *}$ \\
\hline ABA & .29 & $.44^{*}$ & $.75^{* *}$ \\
\hline ABB & .20 & $.38^{*}$ & $.62^{* *}$ \\
\hline SBG & .19 & .14 & $.51^{*}$ \\
\hline SBT & $.65^{* *}$ & $.46^{*}$ & $.63^{* *}$ \\
\hline SBF & $.56^{*}$ & $.43^{*}$ & $.55^{* *}$ \\
\hline SBK & .08 & .27 & .29 \\
\hline SBGK & -.04 & .01 & .05 \\
\hline SBVA & $.64^{*}$ & $.55^{* *}$ & .20 \\
\hline SBW & $.75^{* *}$ & .30 & .40 \\
\hline AB & .22 & $.61^{* *}$ & $.80^{* *}$ \\
\hline BK & $.55^{*}$ & $.45^{* *}$ & .24 \\
\hline
\end{tabular}

Tabelle 4: Interraterreliabilität zu verschiedenen Messzeitpunkten

Für alle signifikanten Reliabilitäten (rot markiert) ist eine Interpretation sinnvoll. Aufgrund der Notwendigkeit, in einer weiteren Studie mehr Experten für das Expertenrating zu gewinnen, werden alle Auswertungen hier nur beispielhaft ausgeführt. Die restlichen (nicht signifikanten Reliabilitäten) sind bei der Interpretation mit Vorsicht zu behandeln.

\subsubsection{Bewegungs/ernen über 3 Messzeitpunkte (Gesamtskala)}

Es werden die Ergebnisse für das Bewegungslernen der Versuchsgruppe über 3 Messzeitpunkte (MZP) (Gesamtskala) angegeben (siehe Tab. 5, Abb. 8).

\begin{tabular}{|c|c|c|}
\hline \multicolumn{3}{|c|}{ Mittelwerte (SD) Bewegungslernen } \\
\hline MZP1 & MZP2 & MZP3 \\
\hline $2,65(0,18)$ & $2,54(0,33)$ & $2,18(0,48)$ \\
\hline $\mathrm{N}=9$ & $\mathrm{~N}=24$ & $\mathrm{~N}=21$ \\
\hline
\end{tabular}

Tabelle 5: Mittelwerte (Standardabweichungen) und Fallzahlen für das Bewegungslernen an drei Messzeitpunkten

Dabei ergeben kleinere Werte eine bessere Beherrschung der Tennistechnik. 


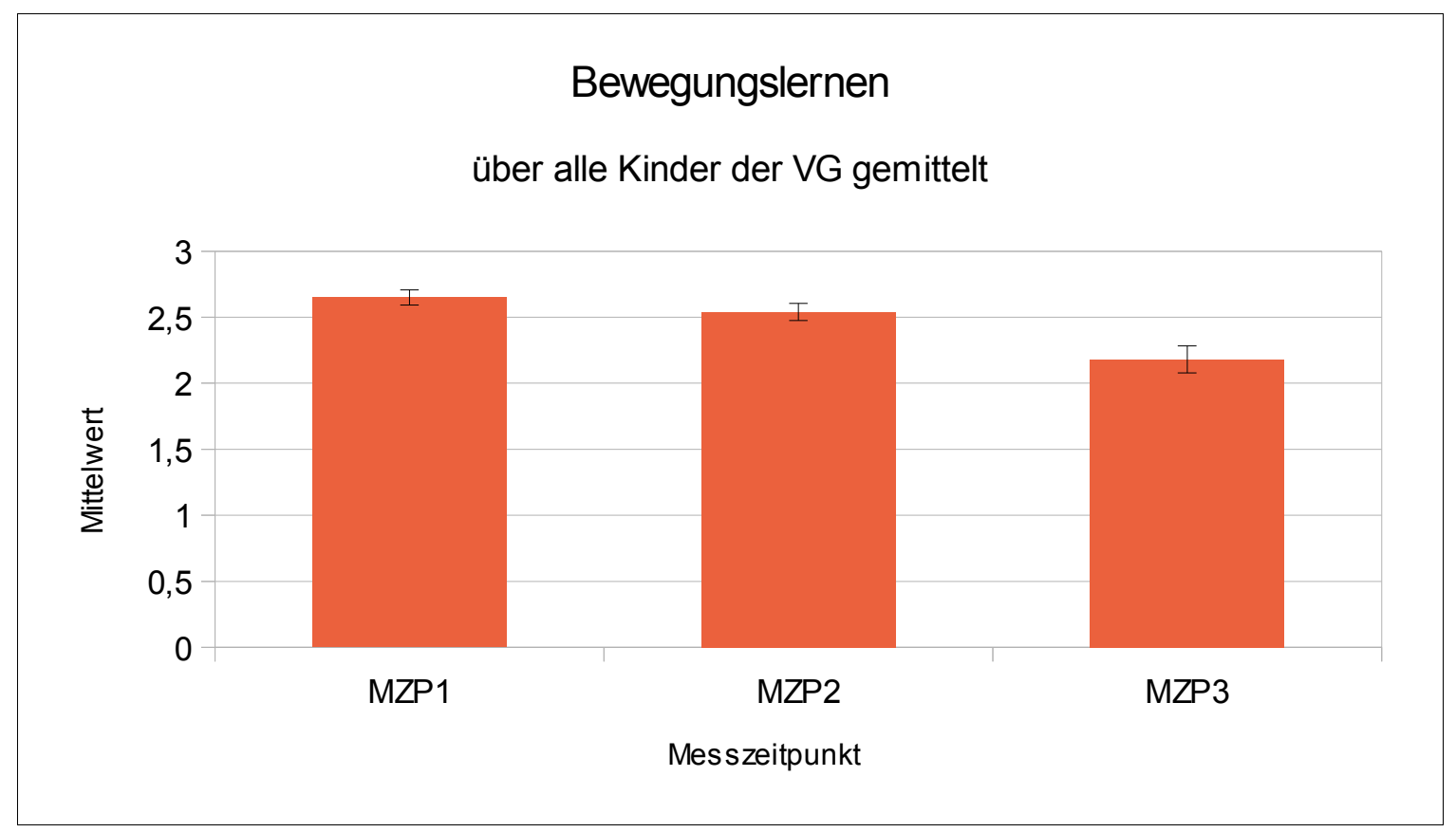

Abbildung 8: Bewegungslernen über drei Messzeitpunkte in der VG

Der T-Test für abhängige Stichproben ergab folgende Ergebnisse:

$\mathrm{Zu}$ den beiden ersten Zeitpunkten MZP1 und MZP2 ändert sich die Tennistechnikbeherrschung als Indikator für das Bewegungslernen nicht signifikant $(T=1,72 ; d f=9 ; p=0.12)$. Im Vergleich dazu ist zum MZP2 der Lernfortschritt durch die signifikanten Unterschiede MZP3 dokumentierbar $(T=3,99 ; \quad d f=21 ; \quad p<.001)$. Auch Veränderungen vom ersten zum dritten Messzeitpunkt werden signifikant $(T=3,78$; $d f=8$; $\mathrm{p}<.01)$.

Problem bei der Berechnung waren die nicht anwesenden Kinder zum MZP1 und MZP3. Das Ersetzen der fehlenden Werte - wie in Holling und Schmitz (2010) empfohlen würde eine zu starke Verzerrung herbeiführen. Deshalb wurde hier darauf verzichtet.

\subsubsection{Bewegungslernen über 3 Messzeitpunkte (Subskalen)}

Die nachstehende Tabelle 6 weist die Ergebnisse des Fragebogens zur Tennistechnik mit seinen Subskalen als Einzelindikatoren für das Bewegungslernen auf. Dabei sind die einzelnen Merkmale im Abkürzungsverzeichnis (siehe Kap. 9.5) beschrieben. 


\begin{tabular}{|l|c|c|c|c|c|c|}
\hline & \multicolumn{2}{|c|}{ MZP1 } & \multicolumn{2}{c|}{ MZP2 } & \multicolumn{2}{c|}{ MZP3 } \\
\hline & Mittel & SD & Mittel & SD & Mittel & SD \\
\hline SP & 2,39 & 0,11 & 2,58 & 0,12 & 2,29 & 0,19 \\
\hline ABA & 2,44 & 0,19 & 2,42 & 0,11 & 2,00 & 0,17 \\
\hline ABB & 2,61 & 0,11 & 2,46 & 0,07 & 2,17 & 0,15 \\
\hline SBG & 2,17 & 0,12 & 1,92 & 0,08 & 1,91 & 0,16 \\
\hline SBT & 2,83 & 0,14 & 2,75 & 0,13 & 2,38 & 0,16 \\
\hline SBF & 2,61 & 0,22 & 3,13 & 0,10 & 2,55 & 0,15 \\
\hline SBK & 2,89 & 0,07 & 2,96 & 0,08 & 2,12 & 0,12 \\
\hline SBGK & 2,61 & 0,14 & 2,67 & 0,08 & 2,19 & 0,10 \\
\hline SBVA & 2,17 & 0,14 & 1,94 & 0,06 & 1,79 & 0,08 \\
\hline SBW & 3,50 & 0,14 & 3,06 & 0,09 & 2,74 & 0,18 \\
\hline AB & 2,67 & 0,19 & 2,44 & 0,13 & 2,17 & 0,19 \\
\hline BK & 2,89 & 0,14 & 2,38 & 0,11 & 2,02 & 0,14 \\
\hline
\end{tabular}

Tabelle 6: Mittelwerte (links) und Standardabweichung (rechts) der Tennismerkmale an drei Messzeitpunkten, MZP1 (N=9), MZP2 (N=24), MZP3 ( $N=21)$

Die folgende Tabelle 7 stellt die Mittelwertvergleiche der MZP 1 und 2 und MZP 2 und 3 sowie MZP 1 und 2 dar. Diese wurden mittels T-Test für abhängige Stichproben durchgeführt. Dabei sind mit rot signifikante Ergebnisse markiert, mit blau werden Trends angegeben. 


\begin{tabular}{|c|c|c|}
\hline \multicolumn{2}{|c|}{ Ergebnisse für das Bewegungslernen } \\
\hline $\begin{array}{c}\text { Vergleich } \\
\text { MZP1 und MZP2 }\end{array}$ & $\begin{array}{c}\text { Vergleich } \\
\text { MZP2 und MZP3 }\end{array}$ & $\begin{array}{c}\text { Vergleich } \\
\text { MZP1 und MZP2 }\end{array}$ \\
\hline SP & SP & SP \\
\hline ABA & ABA & ABA \\
\hline ABB & ABB & ABB \\
\hline SBG & SBG & SBG \\
\hline SBT & SBT & SBT \\
\hline SBF & SBF & SBF \\
\hline SBK & SBK & SBK \\
\hline SBGK & SBGK & SBGK \\
\hline SBVA & SBVA & SBVA \\
\hline SBW & SBW & SBW \\
\hline AB & AB & AB \\
\hline BK & BK & BK \\
\hline
\end{tabular}

Tabelle 7: Ergebnisse des Bewegungslernens über alle Kinder der Versuchsgruppe (rot: signifikant, blau: Trend)

Die T-Tests für abhängige Stichproben ergaben folgende signifikante Ergebnisse für den Vergleich von MZP1 und MZP2:

SBVA1-SVBA2 ( $T=2,40 ; d f=8 ; p=.04)$,

SBW1-SBW2 ( $T=2,44 ; d f=8 ; p=.04)$,

$A B 1-A B 2(T=2,83 ; d f=8 ; p=.02)$;

BK1-BK2 $(T=3,5 ; d f=8 ; p<.01)$.

Einen Trend ergeben folgende Vergleiche:

ABB1-ABB2 $(T=2,29 ; d f=8 ; p=.051)$,

SBK1-SBK2 $(T=2 ; d f=8 ; p=.08)$.

T-Tests (für abhängige Stichproben) ergaben diese signifikanten Resultate für den Vergleich von MZP2 und MZP3:

ABA2-ABA3 $(T=2,37 ; d f=20 ; p=.03)$;

SBT2-SBT3 ( $T=2,31 ; d f=20 ; p=.03)$; 
SBF2-SBF3 ( $T=3,51 ; d f=20 ; p<.01)$;

SBK2-SBK3 ( $T=5,77 ; d f=20 ; p<.001)$;

SBGK2-SBGK3 $(T=4,08 ; d f=20 ; p<.01)$;

BK2-BK3 (T=2,36; $d f=20 ; p=.03)$.

Einen Trend ergibt folgendes Paar: ABB2-ABB3 ( $T=1,76 ; d f=20 ; p=.09)$.

T-Tests für abhängige Stichproben ergaben folgende signifikante Ergebnisse für den Vergleich von MZP1 und MZP3:

SP1-SP3 $(T=4,33 ; d f=7 ; p<.01)$;

ABA1-ABA3 ( $T=7,48 ; d f=7 ; p<.001)$;

ABB1-ABB3 ( $T=3,97 ; d f=7 ; p<.01)$;

SBG1-SBG3 $(T=2,83 ; d f=7 ; p=.03)$;

SBT1-SBT3 $(T=4,32 ; d f=7 ; p<.01)$;

SBK1-SBK3 $(T=6,11 ; d f=7 ; p<.001)$;

SBGK1-SBGK3 $(T=3,42 ; d f=7 ; p=.01)$;

SBVA1-SVBA3 $(T=3,74 ; d f=7 ; p<.01)$;

SBW1-SBW3 $(T=3,99 ; d f=7 ; p<.01)$;

$A B 1-A B 3(T=3 ; d f=7 ; p=.02)$;

BK1-BK3 $(T=8,21 ; d f=7 ; p<.001)$.

Nicht signifikant bleibt allein der Variablenvergleich SBF1-SBF3. 


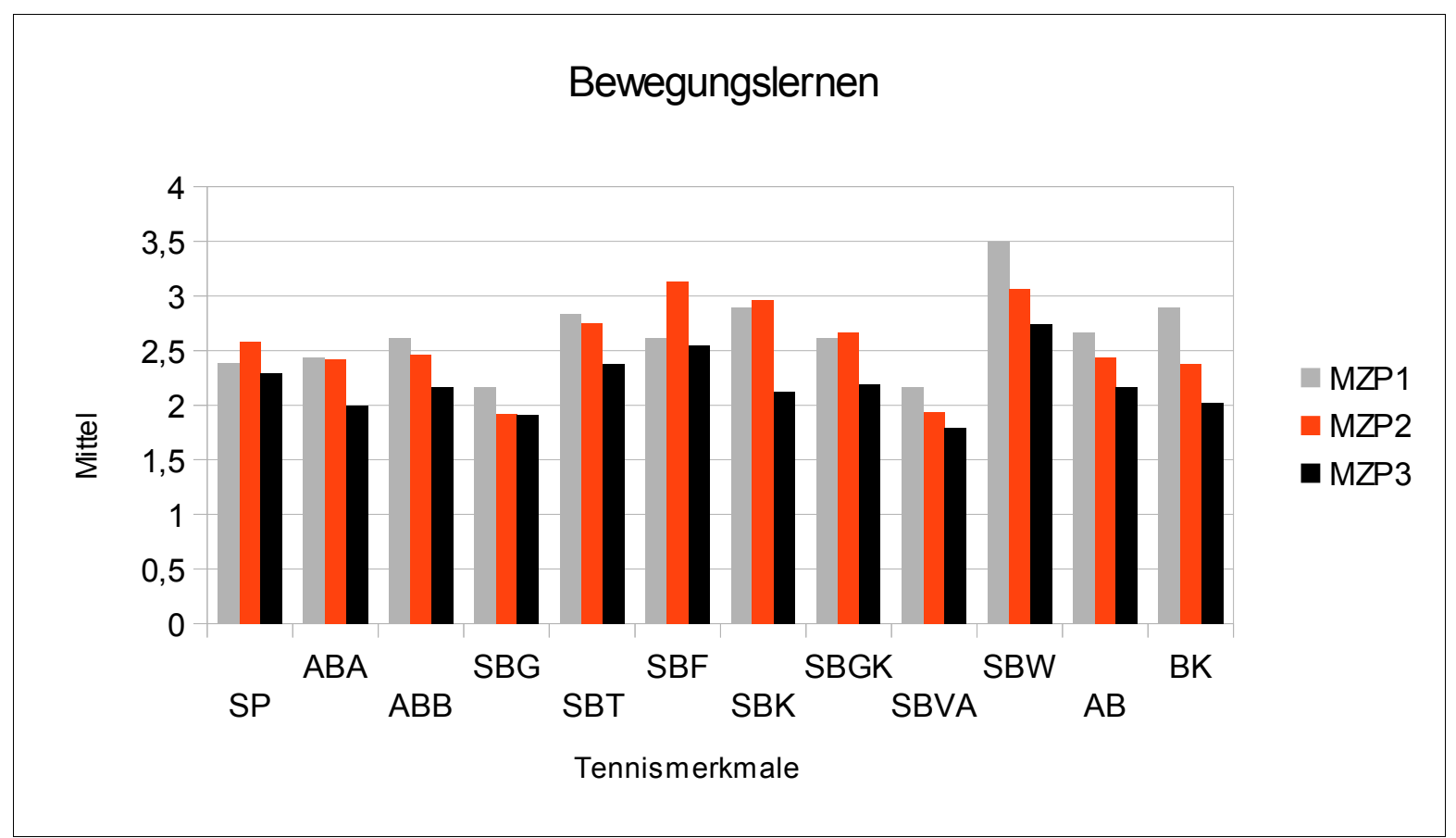

Abbildung 9: Bewegungslernen anhand der verschiedenen Tennismerkmale zu den drei Messzeitpunkten

Die Abbildung 9 stellt die Ergebnisse zum Bewegungslernen grafisch dar.

Kleiner werdende Werte weisen auf eine verbesserte Beherrschung der Tennistechnik und damit auf Bewegungslernen hin.

\subsubsection{Korrelationen zum MZP2 und MZP3 (Gesamtskala)}

Die folgenden zwei Tabellen 8 und 9 geben die Korrelationen für die verschiedenen Teilversuche des Hauptversuchs mit der Gesamtskala des „Fragebogens zur Beherrschung der Tennistechnik" zu den Messzeitpunkten 2 und 3 an. Es ergeben sich bezüglich der Korrelationsrechnung keine signifikanten Ergebnisse, weder zum Messzeitpunkt 2 noch zum Messzeitpunkt 3. Korrelationen wurden mit der Standardmethode nach Pearson berechnet. 


\begin{tabular}{|c|c|c|c|}
\hline \multicolumn{4}{|c|}{ Messzeitpunkt 2 } \\
\hline Teilversuch & Korrelation & Signifikanz & $\mathrm{N}$ \\
\hline 0 & -.11 & .59 & 26 \\
\hline $50 \mathrm{z}$ & -.16 & .43 & 25 \\
\hline $50 \mathrm{~g}$ & .01 & .98 & 19 \\
\hline 25 & -.02 & .94 & 16 \\
\hline $33 \mathrm{z}$ & -.11 & .64 & 20 \\
\hline $33 \mathrm{~g}$ & -.05 & .82 & 20 \\
\hline $67 \mathrm{z}$ & -.18 & .53 & 15 \\
\hline $67 \mathrm{~g}$ & -.12 & .66 & 16 \\
\hline
\end{tabular}

Tabelle 8: Korrelationen von Vorstellungs- und Wahrnehmungsleistungen mit dem Bewegungslernen zum Messzeitpunkt 2

\begin{tabular}{|c|c|c|c|}
\hline \multicolumn{4}{|c|}{ Messzeitpunkt 3 } \\
\hline Teilversuch & Korrelation & Signifikanz & $\mathrm{N}$ \\
\hline 0 & -.17 & .44 & 22 \\
\hline $50 \mathrm{z}$ & .01 & .96 & 21 \\
\hline $50 \mathrm{~g}$ & -.37 & .16 & 16 \\
\hline 25 & .07 & .81 & 13 \\
\hline $33 \mathrm{z}$ & -.17 & .52 & 17 \\
\hline $33 \mathrm{~g}$ & -.32 & .20 & 18 \\
\hline $67 \mathrm{z}$ & -.15 & .62 & 14 \\
\hline $67 \mathrm{~g}$ & -.11 & .72 & 13 \\
\hline
\end{tabular}

Tabelle 9: Korrelationen von Vorstellungs- und Wahrnehmungsleistungen mit dem Bewegungslernen zum Messzeitpunkt 3

Korrelationen werden von wie folgt eingestuft (Bortz, 1993):

- geringe Korrelationen: bis ca. 0.30

- mittlere Korrelationen: ca. 0.30- ca. 0.70

- hohe Korrelationen: ab ca. 0.70

\subsubsection{Korrelationen zum MZP2 (Subskalen)}

Weiterhin wurden die Zusammenhänge zwischen den einzelnen Teilversuchen des Experiments und den Subskalen zum Messzeitpunkt 2 verglichen (siehe folgende Tabellen). Die rote Markierung stellt die signifikante Korrelation dar, die blaue einen Trend (siehe folgende Tabellen 10 bis 21).

Signifikant wurden folgende Korrelationen: die „Schlagbewegung Fußstreckung“ (SBF2) 105 
mit "67z" ergab eine mittlere Korrelation sowie die "Schlagbewegung Wischen“ (SBW2) mit der Basisbedingung "0“ ergab ebenfalls eine mittlere Korrelation. Einen Trend ergab die Beziehung „Schlagbewegung Fußstreckung“ (SBF2) mit „33z“ (durch die Farbe blau gekennzeichnet) (mittlere Korrelation).

Die Korrelationen wurden anhand der Einteilung aus Bortz (1993) bewertet (siehe Kap. 8.3.3.4).

\section{Korrelationen}

\begin{tabular}{|c|c|c|c|c|c|c|c|c|c|}
\hline \multirow{4}{*}{$\overline{\mathrm{SP} 2}$} & & Basis & $50 z$ & $\overline{50 \mathrm{~g}}$ & $\overline{25}$ & $\overline{33 z}$ & $\overline{33 g}$ & $\overline{c 67 z}$ & $67 \mathrm{~g}$ \\
\hline & Korrelation & $-0,238$ & 0,068 & 0,182 & $-0,019$ & $-0,322$ & $-0,005$ & $-0,315$ & $-0,137$ \\
\hline & Signifikanz (2-seitig) & 0,263 & 0,753 & 0,469 & 0,946 & 0,179 & 0,985 & 0,272 & 0,627 \\
\hline & $\mathrm{N}$ & 24 & 24 & 18 & 15 & 19 & 19 & 14 & 15 \\
\hline
\end{tabular}

Tabelle 10: Zusammenhänge zwischen den Subtests des Experiments und dem Tennismerkmal SP2

Korrelationen

\begin{tabular}{|c|c|c|c|c|c|c|c|c|c|}
\hline \multirow{3}{*}{$\overline{\mathrm{ABA} 2}$} & & Basis & $\bar{~} \overline{c 0 z}$ & $\overline{50 \mathrm{~g}}$ & $\overline{25}$ & $33 z$ & $33 g$ & $\overline{67 z z}$ & $67 \mathrm{~g}$ \\
\hline & Korrelation & $-0,105$ & $-0,033$ & $-0,231$ & $-0,130$ & $-0,143$ & $-0,099$ & 0,142 & $-0,480$ \\
\hline & Signifikanz (2-seitig) & 0,624 & 0,878 & 0,356 & 0,646 & 0,559 & 0,687 & 0,629 & 0,070 \\
\hline & $\mathrm{N}$ & 24 & 24 & 18 & 15 & 19 & 19 & 14 & 15 \\
\hline
\end{tabular}

Tabelle 11: Zusammenhänge zwischen den Subtests des Experiments und dem Tennismerkmal ABA2

Korrelationen

\begin{tabular}{|c|c|c|c|c|c|c|c|c|c|}
\hline \multirow{3}{*}{$\overline{\mathrm{ABB} 2}$} & & Basis & $\overline{50 z}$ & 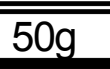 & 25 & $\overline{33 z}$ & $\overline{33 g}$ & $\overline{c 67 z}$ & $67 \mathrm{~g}$ \\
\hline & Korrelation & 0,126 & $-0,317$ & 0,025 & 0,270 & $-0,108$ & $-0,014$ & 0,255 & $-0,508$ \\
\hline & Signifikanz (2-seitig) & 0,557 & 0,131 & 0,923 & 0,331 & 0,659 & 0,956 & 0,379 & 0,053 \\
\hline & $\mathrm{N}$ & 24 & 24 & 18 & 15 & 19 & 19 & 14 & 15 \\
\hline
\end{tabular}

Tabelle 12: Zusammenhänge zwischen den Subtests des Experiments und dem Tennismerkmal ABB2 
Korrelationen

\begin{tabular}{|c|c|c|c|c|c|c|c|c|c|}
\hline & & Basis & $50 z$ & $50 \mathrm{~g}$ & 25 & $33 z$ & $33 g$ & $67 z$ & $67 \mathrm{~g}$ \\
\hline \multirow[t]{3}{*}{ SBG2 } & Korrelation & 0,157 & $-0,186$ & 0,201 & 0,150 & 0,019 & $-0,097$ & $-0,174$ & $-0,326$ \\
\hline & Signifikanz (2-seitig) & 0,464 & 0,385 & 0,423 & 0,592 & 0,939 & 0,693 & 0,551 & 0,236 \\
\hline & $\mathrm{N}$ & 24 & 24 & 18 & 15 & 19 & 19 & 14 & $\underline{5}$ \\
\hline
\end{tabular}

Tabelle 13: Zusammenhänge zwischen den Subtests des Experiments und dem Tennismerkmal SBG2

\section{Korrelationen}

\begin{tabular}{llrrrrrrrr}
\hline \hline & & Basis & \multicolumn{1}{c}{$50 \mathrm{z}$} & $50 \mathrm{~g}$ & 25 & $33 \mathrm{z}$ & $33 \mathrm{~g}$ & $67 \mathrm{z}$ & $67 \mathrm{~g}$ \\
\hline SBT2 & Korrelation & $-0,010$ & $-0,196$ & 0,246 & 0,255 & 0,148 & $-0,050$ & 0,035 & $-0,245$ \\
& Signifikanz (2-seitig) & 0,961 & 0,358 & 0,325 & 0,358 & 0,545 & 0,839 & 0,905 & 0,379 \\
& $\mathrm{~N}$ & 24 & 24 & 18 & 15 & 19 & 19 & 14 & 15 \\
\hline \hline
\end{tabular}

Tabelle 14: Zusammenhänge zwischen den Subtests des Experiments und dem Tennismerkmal SBT2

\begin{tabular}{|c|c|c|c|c|c|c|c|c|c|}
\hline & & Basis & $50 z$ & $50 \mathrm{~g}$ & 25 & $33 z$ & $33 g$ & $67 z$ & $67 \mathrm{~g}$ \\
\hline \multirow[t]{3}{*}{$\overline{\text { SBF2 }}$} & Korrelation & 0,047 & 0,029 & 0,011 & $-0,116$ & 0,392 & 0,107 & $-0,587$ & 0,201 \\
\hline & Signifikanz (2-seitig) & 0,828 & 0,892 & 0,964 & 0,680 & 0,097 & 0,662 & 0,027 & 0,471 \\
\hline & $\mathrm{N}$ & 24 & 24 & 18 & 15 & 19 & 19 & 14 & 15 \\
\hline
\end{tabular}

Tabelle 15: Zusammenhänge zwischen den Subtests des Experiments und dem Tennismerkmal SBF2

\section{Korrelationen}

\begin{tabular}{|c|c|c|c|c|c|c|c|c|c|}
\hline & & Basis & $50 z$ & $\overline{\overline{50 \mathrm{~g}}}$ & 25 & $\overline{33 z}$ & $33 \mathrm{~g}$ & $\overline{67 z}$ & $67 \mathrm{~g}$ \\
\hline \multirow[t]{3}{*}{ SBK2 } & Korre & $-0,054$ & $-0,294$ & $-0,191$ & $-0,337$ & $-0,285$ & $-0,059$ & $-0,152$ & $-0,207$ \\
\hline & Signifikanz (2 & 0,802 & 0,163 & 0,449 & 0,219 & 0,237 & 0,811 & 0,603 & 0,459 \\
\hline & $\mathrm{N}$ & 24 & 24 & 18 & 15 & 19 & 19 & 14 & 15 \\
\hline
\end{tabular}

Tabelle 16: Zusammenhänge zwischen den Subtests des Experiments und dem Tennismerkmal SBK2 
Korrelationen

\begin{tabular}{|c|c|c|c|c|c|c|c|c|c|}
\hline & & Basis & $50 z$ & $50 \mathrm{~g}$ & 25 & $33 z$ & $33 \mathrm{~g}$ & $67 z$ & $67 \mathrm{~g}$ \\
\hline \multirow[t]{3}{*}{ SBGK2 } & Korrel & $-0,163$ & $-0,153$ & $-0,204$ & $-0,254$ & $-0,246$ & $-0,162$ & $-0,409$ & $-0,326$ \\
\hline & Signifikanz (2-seitig) & 0,447 & 0,474 & 0,416 & 0,360 & 0,310 & 0,507 & 0,147 & 0,236 \\
\hline & $\mathrm{N}$ & 24 & 24 & 18 & 15 & 19 & 19 & 14 & 5 \\
\hline
\end{tabular}

Tabelle 17: Zusammenhänge zwischen den Subtests des Experiments und dem Tennismerkmal SBGK2

\section{Korrelationen}

\begin{tabular}{|c|c|c|c|c|c|c|c|c|c|}
\hline \multirow{4}{*}{$\overline{\text { SBVA2 }}$} & & Basis & $50 z$ & $\overline{50 \mathrm{~g}}$ & 25 & 333 & $33 \mathrm{~g}$ & $\overline{c 67 z}$ & $\overline{679 \mathrm{~g}}$ \\
\hline & Korrelation & 0,229 & $-0,137$ & 0,004 & 0,012 & 0,193 & $-0,009$ & $-0,149$ & 0,074 \\
\hline & Signifikanz (2-seitig) & 0,283 & 0,524 & 0,988 & 0,967 & 0,429 & 0,971 & 0,611 & 0,792 \\
\hline & $\mathrm{N}$ & 24 & 24 & 18 & 15 & 19 & 19 & 14 & 15 \\
\hline
\end{tabular}

Tabelle 18: Zusammenhänge zwischen den Subtests des Experiments und dem Tennismerkmal SBVA2

Korrelationen

\begin{tabular}{llrrrrrrrr}
\hline \hline & & Basis & $50 z$ & $50 \mathrm{~g}$ & 25 & $33 \mathrm{z}$ & $33 \mathrm{~g}$ & $67 \mathrm{z}$ & \multicolumn{1}{c}{$67 \mathrm{~g}$} \\
\hline SBW2 & Korrelation & $-0,578$ & $-0,256$ & 0,219 & 0,182 & $-0,254$ & $-0,379$ & $-0,276$ & 0,027 \\
& Signifikanz (2-seitig) & 0,003 & 0,227 & 0,382 & 0,517 & 0,294 & 0,110 & 0,339 & 0,923 \\
& $\mathrm{~N}$ & 24 & 24 & 18 & 15 & 19 & 19 & 14 & 15 \\
\hline \hline
\end{tabular}

Tabelle 19: Zusammenhänge zwischen den Subtests des Experiments und dem Tennismerkmal SBW2

Korrelationen

\begin{tabular}{|c|c|c|c|c|c|c|c|c|c|}
\hline & & Basis & $50 z$ & $50 \mathrm{~g}$ & 25 & $33 z$ & $33 \mathrm{~g}$ & $67 z$ & $67 \mathrm{~g}$ \\
\hline \multirow[t]{3}{*}{$\overline{\mathrm{AB} 2}$} & Korrelation & $-0,052$ & $-0,060$ & $-0,108$ & 0,144 & 0,043 & $-0,013$ & $-0,018$ & $-0,404$ \\
\hline & Signifikanz (2-seitig) & 0,810 & 0,781 & 0,670 & 0,608 & 0,862 & 0,958 & 0,952 & 0,136 \\
\hline & $\mathrm{N}$ & 24 & 24 & 18 & 15 & 19 & 19 & 14 & 15 \\
\hline
\end{tabular}

Tabelle 20: Zusammenhänge zwischen den Subtests des Experiments und dem Tennismerkmal AB2 


\begin{tabular}{|c|c|c|c|c|c|c|c|c|c|}
\hline & & Basis & $50 z$ & $\overline{50 \mathrm{~g}}$ & 25 & $33 z$ & $33 g$ & $67 z$ & $67 g$ \\
\hline \multirow[t]{3}{*}{ BK2 } & Korrelation & $-0,147$ & $-0,232$ & 0,261 & 0,077 & $-0,240$ & $-0,126$ & 0,000 & $-0,287$ \\
\hline & Signifikanz (2-seitig) & 0,492 & 0,275 & 0,295 & 0,786 & 0,322 & 0,608 & 1,000 & 0,300 \\
\hline & $\mathrm{N}$ & 24 & 24 & 18 & 15 & 19 & 19 & 14 & 15 \\
\hline
\end{tabular}

Tabelle 21: Zusammenhänge zwischen den Subtests des Experiments und dem Tennismerkmal BK2

\subsubsection{Korrelationen zum MZP3 (Subskalen)}

Anschließend wurden die Korrelationen zwischen den einzelnen Teilversuchen des Versuchs und den Subskalen zum Messzeitpunkt 3 berechnet (siehe Tabellen 22 bis 33). Es wurden folgende Zusammenhänge signifikant (siehe rote Markierung): "Schlagbewegung Griffhaltung“ (SBG3) mit dem Teilversuch „33g“ ergab eine mittlere Korrelation, die "Schlagbewegung Wischen“ (SBW3) mit „50g“ ergab eine hohe Korrelation und die "Bewegungskonstanz" (BK3) mit der Basisbedingung ergab eine mittlere Korrelation. Einen Trend gab es bei den Korrelationen „Schlagbewegung Griffhaltung“ (SBG3) mit dem Teilversuch „67z“, „Schlagbewegung Wischen“ (SBW3) mit „33g“ mit jeweils mittlerer Ausprägung.

Die Korrelationen wurden anhand der Einteilung von Bortz (1993) bewertet (siehe Kap. 8.3.3.4).

Die rote Markierung stellt die signifikante Korrelation dar, die blaue den Trend (siehe folgende Tabellen 22 bis 33 ).

Korrelationen

\begin{tabular}{|c|c|c|c|c|c|c|c|c|c|}
\hline & & Basis & $\overline{50 z}$ & $\overline{50 \mathrm{~g}}$ & 25 & $\overline{33 z}$ & $33 \mathrm{~g}$ & $\overline{667 z}$ & $\overline{67 g}$ \\
\hline \multirow[t]{3}{*}{ SP3 } & $\begin{array}{l}\text { Korrelation nach } \\
\text { Pearson }\end{array}$ & $-0,229$ & $-0,227$ & $-0,100$ & 0,21 & $-0,260$ & $-0,319$ & $-0,06$ & $-0,125$ \\
\hline & Signifikanz (2-seitig) & 0,318 & 0,322 & 0,713 & 0,488 & 0,314 & 0,197 & 0,835 & 0,683 \\
\hline & $\mathrm{N}$ & 21 & 21 & 16 & 13 & 17 & 18 & 14 & 13 \\
\hline
\end{tabular}

Tabelle 22: Zusammenhänge zwischen den Subtests des Experiments und dem Tennismerkmal SP3 
Korrelationen

\begin{tabular}{lrlrrrrrr}
\hline \hline & Basis & \multicolumn{1}{c}{$50 \mathrm{z}$} & $50 \mathrm{~g}$ & 25 & \multicolumn{1}{c}{$33 \mathrm{z}$} & \multicolumn{1}{c}{$33 \mathrm{~g}$} & $67 \mathrm{z}$ & $67 \mathrm{~g}$ \\
\hline ABA3 Korrelation nach & $-0,074$ & $-0,018$ & $-0,156$ & 0,013 & $-0,411$ & $-0,296$ & 0,014 & $-0,252$ \\
Pearson & & & & & & & & \\
Signifikanz (2-seitig) & 0,749 & 0,940 & 0,565 & 0,966 & 0,101 & 0,233 & 0,963 & 0,406 \\
$\mathrm{~N}$ & 21 & 21 & 16 & 13 & 17 & 18 & 14 & 13 \\
\hline \hline
\end{tabular}

Tabelle 23: Zusammenhänge zwischen den Subtests des Experiments und dem Tennismerkmal ABA3

Korrelationen

\begin{tabular}{lrlrrrrrr}
\hline \hline & Basis & $50 z$ & $50 \mathrm{~g}$ & \multicolumn{1}{c}{25} & $33 \mathrm{z}$ & $33 \mathrm{~g}$ & \multicolumn{1}{c}{$67 \mathrm{z}$} & $67 \mathrm{~g}$ \\
\hline ABB3 Korrelation nach & $-0,105$ & $-0,091$ & $-0,257$ & 0,308 & 0,004 & $-0,162$ & 0,188 & $-0,162$ \\
Pearson & & & & & & \\
Signifikanz (2-seitig) & 0,651 & 0,695 & 0,337 & 0,305 & 0,988 & 0,521 & 0,519 & 0,597 \\
$\mathrm{~N}$ & 21 & 21 & 16 & 13 & 17 & 18 & 14 & 13 \\
\hline \hline
\end{tabular}

Tabelle 24: Zusammenhänge zwischen den Subtests des Experiments und dem Tennismerkmal ABB3

Korrelationen

\begin{tabular}{|c|c|c|c|c|c|c|c|c|}
\hline & Basis & $\overline{\overline{50 z}}$ & $\overline{750 \mathrm{~g}}$ & 25 & 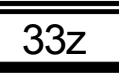 & $\overline{733 g}$ & 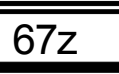 & 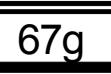 \\
\hline $\begin{array}{c}\text { SBG3 Korrelation nach } \\
\text { Pearson }\end{array}$ & $-0,09$ & $-0,03$ & $-0,18$ & 0,44 & 0,06 & $-0,59$ & $-0,48$ & 0,14 \\
\hline Signifikanz (2-seitig) & 0,696 & 0,885 & 0,508 & 0,128 & 0,824 & 0,010 & 0,083 & 0,651 \\
\hline $\mathrm{N}$ & 21 & 21 & 16 & 13 & 17 & 18 & 14 & 13 \\
\hline
\end{tabular}

Tabelle 25: Zusammenhänge zwischen den Subtests des Experiments und dem Tennismerkmal SBG3

Korrelationen

\begin{tabular}{lllllllll}
\hline \hline & Basis & $50 z$ & $50 \mathrm{~g}$ & \multicolumn{1}{c}{25} & \multicolumn{1}{c}{$33 \mathrm{z}$} & $33 \mathrm{~g}$ & $67 \mathrm{z}$ & $67 \mathrm{~g}$ \\
\hline SBT3 Korrelation nach & $-0,289$ & $-0,056$ & $-0,233$ & $-0,108$ & $-0,150$ & $-0,139$ & 0,300 & $-0,238$ \\
Pearson & & & & & & & & \\
Signifikanz (2-seitig) & 0,204 & 0,811 & 0,384 & 0,726 & 0,566 & 0,583 & 0,298 & 0,433 \\
$\mathrm{~N}$ & 21 & 21 & 16 & 13 & 17 & 18 & 14 & 13 \\
\hline \hline
\end{tabular}

Tabelle 26: Zusammenhänge zwischen den Subtests des Experiments und dem Tennismerkmal SBT3 
Korrelationen

\begin{tabular}{llrrrrrrr}
\hline \hline & Basis & $50 z$ & $50 \mathrm{~g}$ & 25 & $33 z$ & $33 g$ & $67 z$ & $67 g$ \\
\hline SBF3 Korrelation nach & 0,037 & 0,281 & $-0,122$ & 0,069 & 0,181 & $-0,098$ & $-0,444$ & 0,357 \\
Pearson & & 0,218 & 0,652 & 0,822 & 0,486 & 0,698 & 0,111 & 0,230 \\
Signifikanz (2-seitig) & 0,873 & 0,21 & 16 & 13 & 17 & 18 & 14 & 13 \\
N & 21 & 21 & 16 &
\end{tabular}

Tabelle 27: Zusammenhänge zwischen den Subtests des Experiments und dem Tennismerkmal SBF3

Korrelationen

\begin{tabular}{|c|c|c|c|c|c|c|c|c|}
\hline & Basis & $50 z$ & $50 \mathrm{~g}$ & 25 & $33 z$ & $33 g$ & $67 z$ & $67 g$ \\
\hline $\begin{array}{l}\text { SBGK Korrelation nach } \\
3 \quad \text { Pearson }\end{array}$ & 0,028 & 0,132 & $-0,148$ & 0,064 & 0,038 & $-0,037$ & $-0,333$ & 0,119 \\
\hline Signifikanz (2-seitig) & 0,904 & 0,570 & 0,584 & 0,836 & 0,884 & 0,885 & 0,244 & 0,698 \\
\hline $\mathrm{N}$ & 21 & 21 & 16 & 13 & 17 & 18 & 14 & 13 \\
\hline
\end{tabular}

Tabelle 28: Zusammenhänge zwischen den Subtests des Experiments und dem Tennismerkmal SBGK3

Korrelationen

\begin{tabular}{|c|c|c|c|c|c|c|c|c|}
\hline & Basis & $\overline{50 z}$ & $\overline{50 \mathrm{~g}}$ & $\overline{25}$ & $\overline{\overline{33 z}}$ & $33 \mathrm{~g}$ & $67 z$ & $67 \mathrm{~g}$ \\
\hline $\begin{array}{l}\text { SBVA Korrelation nach } \\
3 \quad \text { Pearson }\end{array}$ & $-0,066$ & $-0,351$ & $-0,355$ & 0,058 & $-0,238$ & $-0,046$ & 0,194 & $-0,250$ \\
\hline Signifikanz (2-seitig) & 0,775 & 0,119 & 0,177 & 0,852 & 0,358 & 0,856 & 0,506 & 0,411 \\
\hline $\mathrm{N}$ & 21 & 21 & 16 & 13 & 17 & 18 & 14 & 13 \\
\hline
\end{tabular}

Tabelle 29: Zusammenhänge zwischen den Subtests des Experiments und dem Tennismerkmal SBVA3

Korrelationen

\begin{tabular}{lrrrrrrrr}
\hline \hline & Basis & $50 z$ & $50 \mathrm{~g}$ & \multicolumn{1}{c}{25} & \multicolumn{1}{c}{$33 \mathrm{z}$} & $33 \mathrm{~g}$ & $67 \mathrm{z}$ & $67 \mathrm{~g}$ \\
\hline SBK3 Korrelation nach & 0,085 & 0,211 & $-0,400$ & $-0,209$ & $-0,143$ & 0,100 & $-0,455$ & 0,043 \\
Pearson & & & & & & & \\
Signifikanz (2-seitig) & 0,715 & 0,358 & 0,124 & 0,493 & 0,583 & 0,694 & 0,102 & 0,890 \\
$\mathrm{~N}$ & 21 & 21 & 16 & 13 & 17 & 18 & 14 & 13 \\
\hline \hline
\end{tabular}

Tabelle 30: Zusammenhänge zwischen den Subtests des Experiments und dem Tennismerkmal ABK3 


\begin{tabular}{|c|c|c|c|c|c|c|c|c|c|}
\hline & & Basis & $\overline{50 z}$ & $\overline{50 \mathrm{~g}}$ & 25 & $\overline{\overline{33 z}}$ & $\overline{33 g}$ & $\overline{\overline{67 z}}$ & $\overline{67 g}$ \\
\hline \multirow[t]{3}{*}{$\begin{array}{l}\text { SBW } \\
3\end{array}$} & $\begin{array}{l}\text { Korrelation nach } \\
\text { Pearson }\end{array}$ & $-0,115$ & $-0,035$ & $-0,731$ & $-0,070$ & $-0,013$ & $-0,407$ & $-0,311$ & $-0,297$ \\
\hline & Signifikanz (2-seitig) & 0,619 & 0,879 & 0,001 & 0,819 & 0,961 & 0,094 & 0,279 & 0,324 \\
\hline & $\mathrm{N}$ & 21 & 21 & 16 & 13 & 17 & 18 & 14 & 13 \\
\hline
\end{tabular}

Tabelle 31: Zusammenhänge zwischen den Subtests des Experiments und dem Tennismerkmal SBW3

Korrelationen

\begin{tabular}{|c|c|c|c|c|c|c|c|c|c|}
\hline & & Basis & $50 z$ & $50 \mathrm{~g}$ & 25 & $33 z$ & $33 g$ & $67 z$ & $67 g$ \\
\hline \multirow[t]{3}{*}{ AB3 } & $\begin{array}{l}\text { Korrelation nach } \\
\text { Pearson }\end{array}$ & $-0,100$ & 0,263 & $-0,394$ & $-0,332$ & $-0,331$ & $-0,086$ & 0,012 & $-0,217$ \\
\hline & Signifikanz (2-seitig) & 0,667 & 0,250 & 0,131 & 0,268 & 0,195 & 0,736 & 0,967 & 0,476 \\
\hline & $\mathrm{N}$ & 21 & 21 & 16 & 13 & 17 & 18 & 14 & 13 \\
\hline
\end{tabular}

Tabelle 32: Zusammenhänge zwischen den Subtests des Experiments und dem Tennismerkmal AB3

Korrelationen

\begin{tabular}{|c|c|c|c|c|c|c|c|c|c|}
\hline \multirow{2}{*}{ BK3 } & & Basis & $\overline{50 z}$ & $\overline{50 \mathrm{~g}}$ & 25 & $\overline{33 z}$ & $\overline{33 g}$ & $\overline{667 z}$ & $\overline{67 g}$ \\
\hline & $\begin{array}{l}\text { Korrelation nach } \\
\text { Pearson }\end{array}$ & $-0,467$ & $-0,037$ & $-0,054$ & 0,327 & $-0,157$ & $-0,339$ & 0,031 & 0,137 \\
\hline & Signifikanz (2-seitig) & 0,033 & 0,873 & 0,842 & 0,275 & 0,547 & 0,169 & 0,915 & 0,655 \\
\hline & $\mathrm{N}$ & 21 & 21 & 16 & 13 & 17 & 18 & 14 & 13 \\
\hline
\end{tabular}

Tabelle 33: Zusammenhänge zwischen den Subtests des Experiments und dem Tennismerkmal BK3 


\subsubsection{Einzelfallanalyse zum Bewegungslernen}

Eine Einzelfallanalyse kann Aufschluss über das Niveau der Fertigkeiten und den Lernzuwachs beim jeweiligen Kind geben. Ein Vergleich mit anderen Lernprofilen (siehe Anhang 9.2) macht interindividuelle (Lern-)Unterschiede deutlich.

Folgende Einzelfallanalysen wurden durchgeführt (siehe Abb. 10 und Kap. 9.2). Dabei entsprechen niedrige Werte einer besseren Leistung als hohe. Die 3 Messzeitpunkte ergeben einen nicht linearen Zeitstrahl: MZP 1: Februar 2004 ,MZP 2: Oktober/November 2004, MZP 3: September 2005. Das bedeutet, die Veränderungen von MZP 2 zu MZP 3 haben sich in einem größeren Zeitraum abgespielt als diejenigen zwischen den ersten beiden Messzeitpunkten.

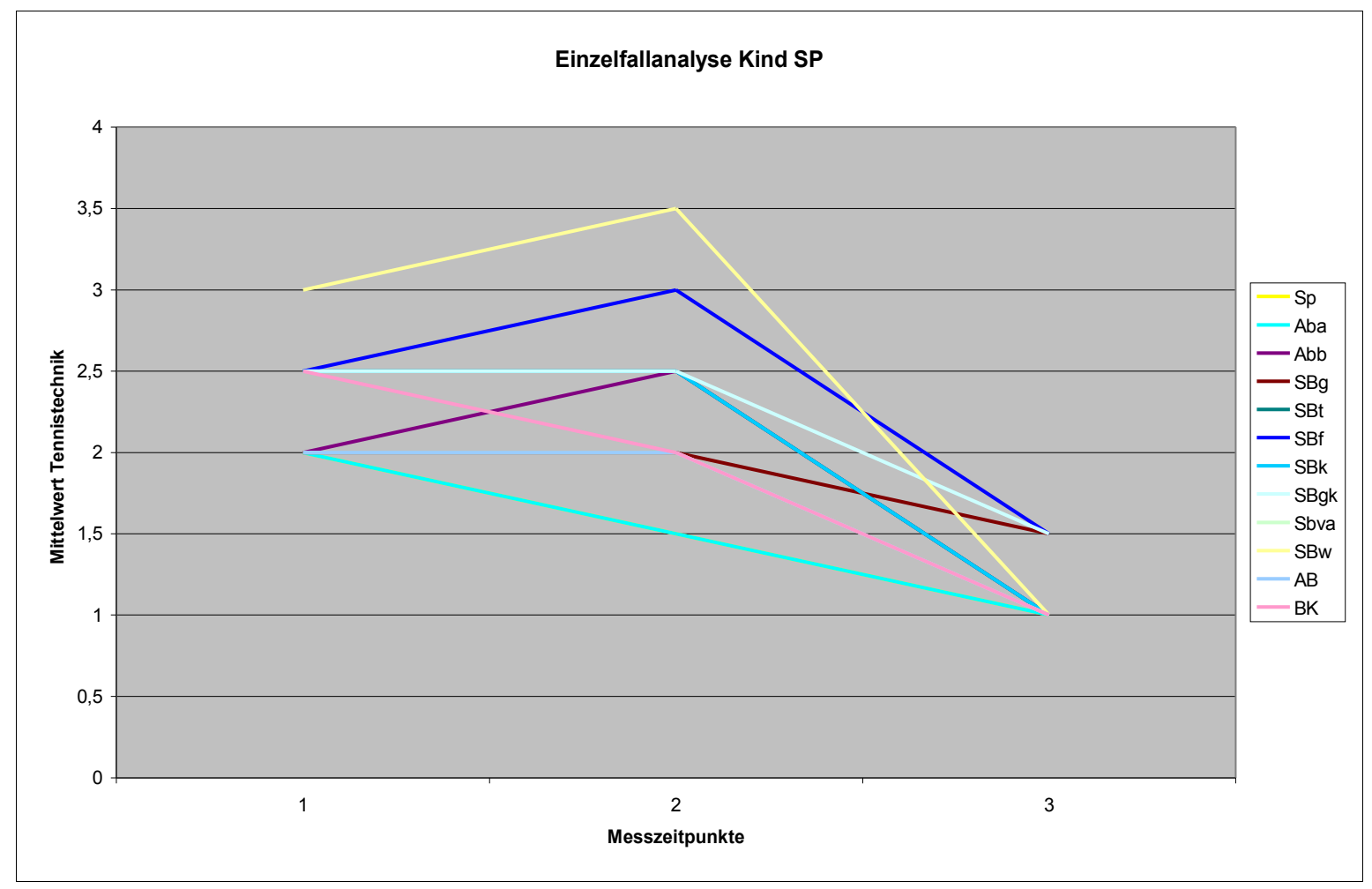

Abbildung 10: Entwicklung der Tennistechnik über 3 Messzeitpunkte bei Kind SP

Die Einzelfallbetrachtungen im Kap. 9.2 machen deutlich, dass das Lernen von Bewegungen sehr unterschiedliche Verläufe bei einzelnen Kindern annehmen kann. Eine gute Leistung zu einem Messzeitpunkt kann zudem auch wieder schlechter werden. Außerdem kann das Niveau zu den Anfangs-, Zwischen- und Endmesszeitpunkten jeweils unterschiedlich sein (siehe Steigungswinkel der Geraden). Sicherlich hat die Selektion der Stichprobe (hier gemeint sind die Filmsequenzen der Kinder, die den Experten gezeigt wurden) einen Einfluss auf die Ergebnisse. Dennoch kann gezeigt 
werden, dass Bewegungslernen stattfindet und dass es verschiedene Indikatoren hierfür gibt (z.B. hier verschiedene Merkmale der Tennistechnik), welche in einem Expertenrating ermittelt wurden. Dabei sind solche Werte wie die Fußstreckung und die Vorwärts-Aufwärts-Bewegung weniger gut geeignete Lernindikatoren (siehe Kap. 8.3.3.8). Alle anderen waren zumindest im Zeitraum der ersten und dritten Messung von Bedeutung.

Es wurde auch eine Rangfolge der Kinder hinsichtlich der erzielten Werte im Bewegungslernen abgebildet (siehe Anhang 9.4).

\subsubsection{Merkmale der Tennistechnik als Lernindikatoren}

Die Merkmale der Tennistechnik können als Lernindikatoren für das Bewegungslernen angesehen werden und wurden in der folgenden Tabelle 34 dargestellt. Eine Rangfolge nahe eins bedeutet, dass es eher leicht ist, dieses Tennismerkmal zu erwerben. Lernindikatoren geben an, ob die Merkmale eher leichter oder eher schwerer erlernt werden können.

\begin{tabular}{|c|c|c|c|c|c|c|c|c|c|c|c|c|c|}
\hline \multicolumn{10}{|c|}{ Messzeitpunkt 1 } \\
\hline & SP1 & ABA1 & ABB1 & SBG1 & SBT1 & SBF1 & SBK1 & SBGK1 & SBVA1 & SBW1 & AB1 & BK1 \\
\hline Mittel & 2,3 & 2,33 & 2,53 & 2,17 & 2,77 & 2,7 & 2,77 & 2,6 & 2,1 & 3,1 & 2,6 & 2,9 \\
\hline Rang & 3 & 4 & 5 & 2 & 9 & 8 & 9 & 6 & 1 & 11 & 6 & 10 \\
\hline \multicolumn{10}{|c|}{ Messzeitpunkt 2 } \\
\hline & SP2 & ABA2 & ABB2 & SBG2 & SBT2 & SBF2 & SBK2 & SBGK2 & SBVA2 & SBW2 & AB2 & BK2 \\
\hline Mittel & 2,47 & 2,36 & 2,36 & 1,89 & 2,67 & 3,12 & 2,91 & 2,7 & 2 & 3,03 & 2,39 & 2,3 \\
\hline Rang & 7 & 3 & 3 & 1 & 8 & 12 & 10 & 9 & 2 & 11 & 6 & 5 \\
\hline \multicolumn{10}{|c|}{ Messzeitpunkt 3 } & & & \\
\hline & SP3 & ABA3 & ABB3 & SBG3 & SBT3 & SBF3 & SBK3 & SBGK3 & SBVA3 & SBW3 & AB3 & BK3 \\
\hline Mittel & 2,25 & 1,92 & 2,15 & 1,87 & 2,35 & 2,63 & 2,13 & 2,19 & 1,83 & 2,67 & 2,12 & 2,1 \\
\hline Rang & 9 & 3 & 7 & 2 & 10 & 11 & 6 & 8 & 1 & 12 & 5 & 4 \\
\hline
\end{tabular}

Tabelle 34: durchschnittliche Ausprägung der Tennistechnik und Rangfolge der Mittelwerte zu den drei Messzeitpunkten (durch das Expertenrating ermittelt) (Anmerkung: hier sind die genauen Mittelwerte eingetragen, während bei den T-Tests oben ein Fallausschluss die Mittelwerte beeinflusst)

Das am schwierigsten auszuführende Merkmal scheint bei fast allen Messzeitpunkten das Wischen zu sein (SBW1, SBW2, SBW3). Am leichtesten ist die Griffhaltung (SBG) und die Vorwärts-Aufwärts-Bewegung (SBVA) zu erlernen. Möglich ist aber auch diese Interpretation, dass die studentischen Experten, die die Tennissequenzen der Kinder 
analysieren und bewerten, bei diesen Merkmalen wie z.B. beim Wischen besonders kritisch zu sein scheinen.

\subsubsection{Diskussion}

Es wird hier in dieser Dissertation angenommen, dass der Erfolg beim Erwerb sportlicher Fertigkeiten anhand kognitiver Leistungen ermittelt werden kann. Somit sollte ein Zusammenhang von Indikatoren für das Bewegungslernen und jenen für Wahrnehmungs- und Vorstellungsleistungen für diese These sprechen.

Aufgrund der Interraterreliabilitäten, die nicht für alle Merkmale des Fragebogens signifikant wurden, ist eine Interpretation nur mit Vorsicht durchzuführen.

Es konnte gezeigt werden, dass Bewegungslernen stattfindet. Insbesondere zum dritten Messzeitpunkt sind Veränderungen auf fast allen Subskalen feststellbar. Dennoch zeigen die Einzelfallanalysen, dass individueller Lernfortschritt mit Rückschritten oder Plateauphasen einhergehen kann. Auch können teilweise große interindividuelle Unterschiede im Verlauf und im Niveau der Leistungen zu den Messzeitpunkten dargestellt werden. Die Darstellung von sogenannten Lernindikatoren zeigt, dass nicht alle Merkmale gleich gut erlernt werden können. Auf das Problem fehlender Werte wurde schon hingewiesen; auf einen Ersatz durch Prozeduren wurde wegen des Problems der zu starken Verzerrung der Ergebnisse verzichtet.

Zusammenhänge zwischen dem Bewegungslernen und Vorstellungen können nur gefunden werden, wenn die einzelnen Subskalen des Fragebogens für die Tennistechnik zur Berechnung herangezogen werden. Die erste Hypothese $(\mathrm{H} 1)$ lässt sich nur in Teilen bestätigen. Denn es ergeben sich in Anbetracht der 24 Messungen (12 Skalen zu 2 Messzeitpunkten) 21 Prozent signifikante Korrelationen (5 Messpaare). Es kann somit in einzelnen Fällen von einem Zusammenhang von Vorstellungen der Bewegungen mit dem Bewegungslernen in der Kindheit ausgegangen werden. Abhängig scheint dies von der Art des verwendeten Teilversuchs des Experiments zu sein. Im Rahmen einer korrelativen Studie ist es allerdings nicht möglich, auf einen Kausalzusammenhang zu schließen. Auch sind Scheinkorrelationen möglich. Dies sind Korrelationen, die von anderen Faktoren bestimmt werden als diejenigen, die in der Gleichung vorhanden sind.

Besonders interessant erscheint zudem die Korrelation von der Bewegungskonstanz zum dritten Messzeitpunkt (BK3) mit der Basisbedingung. Dies könnte ein Hinweis dafür 
sein, dass nicht nur Vorstellungsleistungen das Bewegungslernen beeinflussen, sondern auch Wahrnehmungsprozesse (Bewegungswahrnehmung). Doch auch hier gelten vorsichtige Interpretationen, was den Kausalzusammenhang angeht.

Einzelne Subskalen des Fragebogens zur Tennistechnik, mit dem das Bewegungslernen erfasst wurde, ergaben Zusammenhänge zwischen Vorstellungen und Bewegungslernen bei Kindern. Abhängig scheint dies von der Art des verwendeten Teilversuchs des Experiments zu sein. Kognitive Faktoren (wie die Wahrnehmung und die Vorstellung von Bewegungen) sind demnach beim Erwerb einer sportlichen Fertigkeit in der Kindheit beteiligt. Damit werden Ergebnisse von Feltz und Landers (1983) sowie von Kosslyn, Behrmann und Jeannerod (1995) bestätigt, welche die Rolle der mentalen Vorstellungen für den Fertigkeitserwerb betonen. Die Eingangshyothese bestätigt sich in Teilen. 


\subsection{Studie 2: Einfluss von Erfahrung auf Wahrnehmung und Vorstellung von Bewegungen in der Kindheit}

Es wurde hier der Einfluss von Erfahrung auf die Wahrnehmung und Vorstellung von Bewegungen untersucht. Laut Hypothese haben die Tenniskinder (sie verfügen über visuelle und motorische Erfahrungen bezüglich der Tennisbewegungen) gegenüber Kindern, die nicht diese Erfahrungen besitzen, einen Lösungsvorteil bei den Wahrnehmungs- und Vorstellungsaufgaben.

\subsubsection{Stichprobe}

Zusätzlich zu den 30 Tenniskindern der Versuchsgruppe (siehe Stichprobenbeschreibung in Kap. 8.3.1) wurde eine Kontrollgruppe gesucht, die vergleichbar hinsichtlich des Merkmals Alter war (Parallelisierung). Das Merkmal „Anzahl der ausgeführten Sportarten" wurde nicht kontrolliert, da hier der Einfluss der sportlichen Expertise als Gruppenfaktor erhalten bleiben sollte. In die Kontrollgruppe wurden nur Kinder aufgenommen, die keine Tenniserfahrung hatten, was durch Unterschrift der Eltern bestätigt wurde. Ein Kind der Kontrollgruppe wurde nicht einbezogen, weil es während der Befragung angab, schon einmal Tennis gespielt zu haben.

Die parallelisierte Kontrollgruppe (KG) bestand somit aus 35 Kindern der ersten bis dritten Klasse einer Grundschule in Göttingen. Weiterhin fiel auf, dass die Kinder der Kontrollgruppe häufiger aus Familien mit Migrationshintergrund kamen. Ein Kind aus der Kontrollgruppe verweigerte die Teilnahme.

Es wird die Altersstruktur der Stichprobe aufgeführt (siehe Tab. 35):

\begin{tabular}{|l|c|c|c|c|c|c|}
\hline \multicolumn{6}{|c|}{ Alter Gesamtgruppe- deskriptive Statistik } \\
\hline & N & Min & Max & Mittelwert & $\begin{array}{c}\text { Standard- } \\
\text { abweichung }\end{array}$ & Varianz \\
\hline ALTER0 & 65 & 5,5 & 10,2 & 8,27 & 1,25 & 1,55 \\
\hline ALTER50Z & 57 & 5,5 & 10,2 & 8,23 & 1,23 & 1,51 \\
\hline ALTER50G & 57 & 5,5 & 10,5 & 8,42 & 1,22 & 1,48 \\
\hline
\end{tabular}

Tabelle 35: Alter der Gesamtgruppe in der deskriptiven Statistik zu den einzelnen Teilversuchs (Anmerkung: Alter0=Alter in der Basisbedingung)

Die geringeren Mittelwerte bei den Teilversuchen „67z" und „67g“ waren durch Nichtanwesenheit/Ausfälle bei den älteren Kindern bedingt.

In den folgenden Tabellen 36 und 37 wird das Alter nach Gruppen angegeben: 


\begin{tabular}{|l|c|c|c|c|c|c|}
\hline \multicolumn{7}{|c|}{ Alter in VG- deskriptive Statistik } \\
\hline & N & Min & Max & Mittelwert & $\begin{array}{c}\text { Standard- } \\
\text { abweichung }\end{array}$ & Varianz \\
\hline ALTER0 & 30 & 5,5 & 10,1 & 8,06 & 1,32 & 1,74 \\
\hline ALTER50Z & 29 & 5,5 & 10,2 & 8,14 & 1,33 & 1,76 \\
\hline ALTER50G & 27 & 5,5 & 10,5 & 8,37 & 1,38 & 1,89 \\
\hline
\end{tabular}

Tabelle 36: Alter in der Versuchsgruppe (Anmerkung: Alter0=Alter in der Basisbedingung)

\begin{tabular}{|l|c|c|c|c|c|c|}
\hline \multicolumn{7}{|c|}{ Alter in KG - deskriptive Statistik } \\
\hline & & & & & $\begin{array}{c}\text { Standard- } \\
\text { abweichung }\end{array}$ & Varianz \\
\hline ALTER0 & 35 & 6,4 & 10,2 & 8,46 & 1,16 & 1,40 \\
\hline ALTER50Z & 33 & 6,4 & 10,2 & 8,44 & 1,17 & 1,37 \\
\hline ALTER50G & 35 & 6,5 & 10,2 & 8,49 & 1,09 & 1,19 \\
\hline
\end{tabular}

Tabelle 37: Alter in der Kontrollgruppe (Anmerkung: Alter0=Alter in der Basisbedingung)

Die beiden Gruppen unterscheiden sich laut T-Test für unabhängige Stichproben in den folgenden Variablen nicht:

- Alter0 (Alter zum Test in der Basisbedingung „0“): $\mathrm{T}=-1,31 ; \mathrm{p}=.20$, df= 63

- Alter50z (Alter zum Test „50z"): $T=-.93 ; p=.36, d f=60$

- Alter50g (Alter zum Test „50g“): $\mathrm{T}=-.39 ; \mathrm{p}=.70, \mathrm{df}=60$

Um zunächst zu überprüfen, ob sich die Versuchs- und die Kontrollgruppe hinsichtlich des Merkmals Geschlecht unterscheiden, müsste ein Chi-Quadrat-Test berechnet werden. Der Chi-Quadrat-Test prüft, ob zwei Merkmale voneinander unabhängig sind. Bei kleinen Fallzahlen ist dieser Test jedoch nicht aussagekräftig. Das Geschlechterverhältnis wird deshalb wie folgt beschrieben (siehe Abb. 11): Das Geschlechterverhältnis ist in beiden Gruppen verschieden. 


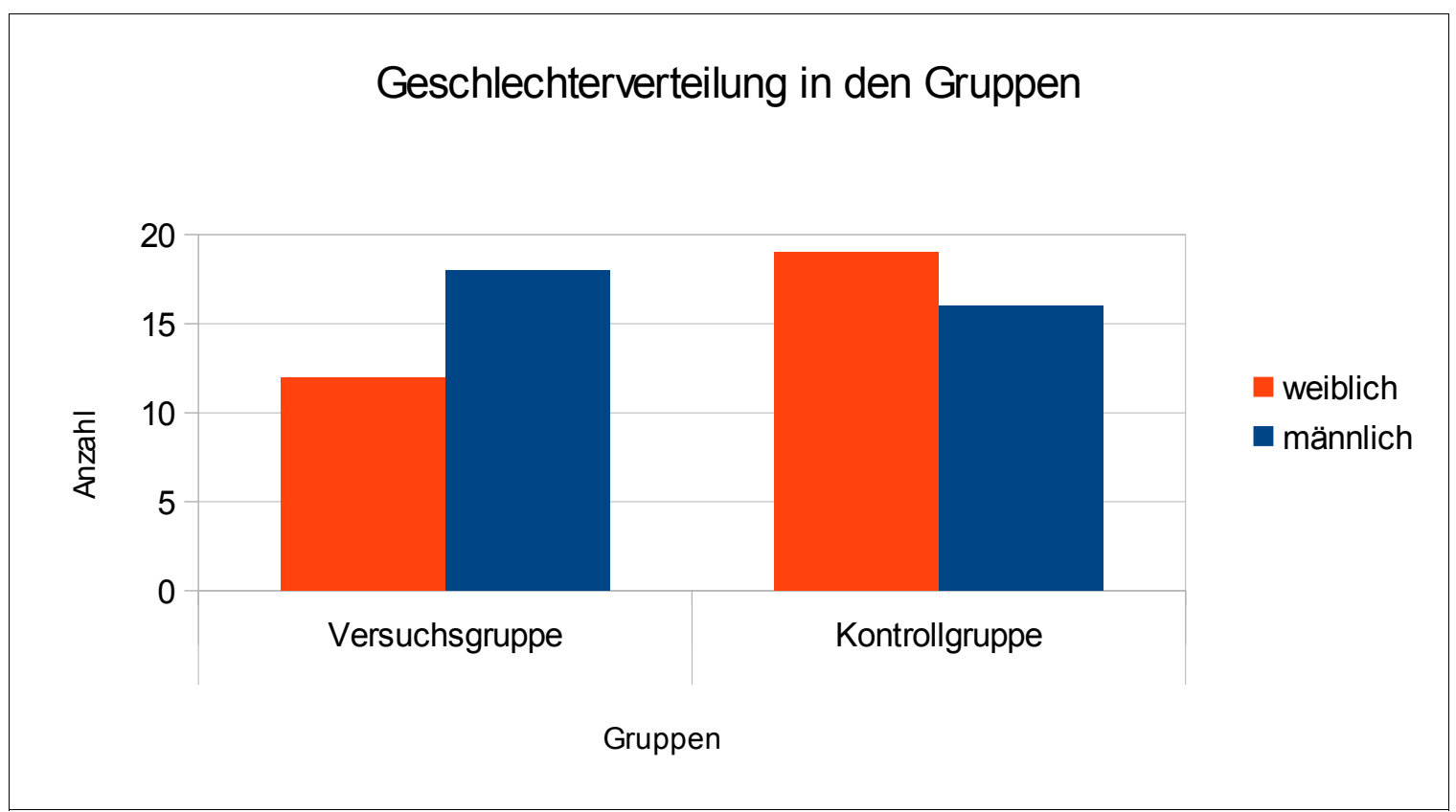

Abbildung 11: Geschlecht nach Gruppen

\subsubsection{Methode}

In der Studie 2 ging es um den möglichen Einfluss von Erfahrung auf die Wahrnehmungs- und Vorstellungsleistung. Dazu wurden die Tenniskinder mit einer Vergleichsgruppe ohne Tenniserfahrung verglichen. Weil die Versuchspersonen den Versuchsgruppen nicht zufällig zugeordnet werden konnten, ergibt sich ein quasiexperimentelles Design.

Die Messung des Bewegungslernens und das Material für die Untersuchung der Wahrnehmungs- und Vorstellungsleistung wurde bereits in der Studie 1 beschrieben (siehe Kap. 8.3.2.1).

Während die Kinder der Versuchsgruppe während des Trainings untersucht wurden, fanden die Versuche bei der Kontrollgruppe während einer Deutschstunde statt, da die Durchführung während des Sportunterrichts nicht möglich war. Der weitere Ablauf entsprach dem des Ablaufes in der Studie 1 (siehe Kap. 8.3.2.2).

\subsubsection{Auswertungsprozedur}

Der Vergleich der Wahrnehmungs- und Vorstellungsleistung mit der Ratewahrscheinlichkeit wurde mittels T-Test (mit einem Testwert von 50) berechnet.

Die Versuchs- und die Kontrollgruppe wurde danach hinsichtlich des Einflusses von Erfahrung auf die Vorstellungsleistung mittels einer Varianzanalyse mit Messwiederholungen verglichen. Der Messwiederholungsfaktor bestand aus den drei 
Versuchsbedingungen (Basisbedingung „0“, „50z und „50g“). Der Faktor Erfahrung war dabei der between-subject-Faktor.

\subsubsection{Ergebnisse der Studie 2}

Die deskriptive Statistik ergab folgende Ergebnisse (siehe Tab. 38):

Deskriptive Statistiken

\begin{tabular}{|c|c|c|c|c|c|}
\hline & GRUPPE & GESCHL & Mittelwert & $\begin{array}{l}\text { Standardab } \\
\text { weichung }\end{array}$ & $\mathrm{N}$ \\
\hline \multirow[t]{9}{*}{ Basisbed } & \multirow[t]{3}{*}{ VG } & männl & 67,30 & 18,159 & 13 \\
\hline & & weibl & 65,83 & 12,711 & 10 \\
\hline & & Gesamt & 66,66 & 15,701 & 23 \\
\hline & \multirow[t]{3}{*}{ KG } & männl & 66,02 & 13,384 & 13 \\
\hline & & weibl & 76,79 & 8,106 & 14 \\
\hline & & Gesamt & 71,60 & 12,067 & 27 \\
\hline & \multirow[t]{3}{*}{ Gesamt } & männl & 66,66 & 15,643 & 26 \\
\hline & & weibl & 72,22 & 11,437 & 24 \\
\hline & & Gesamt & 69,33 & 13,933 & 50 \\
\hline \multirow[t]{9}{*}{$50 Z$} & \multirow[t]{3}{*}{ VG } & männl & 57,062 & 17,9533 & 13 \\
\hline & & weibl & 58,330 & 11,1084 & 10 \\
\hline & & Gesamt & 57,613 & 15,0568 & 23 \\
\hline & \multirow[t]{3}{*}{ KG } & männl & 51,262 & 12,6597 & 13 \\
\hline & & weibl & 67,414 & 10,5862 & 14 \\
\hline & & Gesamt & 59,637 & 14,0587 & 27 \\
\hline & \multirow[t]{3}{*}{ Gesamt } & männl & 54,162 & 15,5045 & 26 \\
\hline & & weibl & 63,629 & 11,5134 & 24 \\
\hline & & Gesamt & 58,706 & 14,4117 & 50 \\
\hline \multirow[t]{9}{*}{$50 \mathrm{G}$} & \multirow[t]{3}{*}{ VG } & männl & 61,546 & 13,5144 & 13 \\
\hline & & weibl & 60,840 & 15,2427 & 10 \\
\hline & & Gesamt & 61,239 & 13,9570 & 23 \\
\hline & \multirow[t]{3}{*}{ KG } & männl & 64,831 & 13,8176 & 13 \\
\hline & & weibl & 79,721 & 14,0927 & 14 \\
\hline & & Gesamt & 72,552 & 15,6495 & 27 \\
\hline & \multirow[t]{3}{*}{ Gesamt } & männl & 63,188 & 13,4950 & 26 \\
\hline & & weibl & 71,854 & 17,1344 & 24 \\
\hline & & Gesamt & 67,348 & 15,8066 & 50 \\
\hline
\end{tabular}

Tabelle 38: deskriptive Statistik für die Faktoren Gruppe, Geschlecht und Versuchsbedingung hinsichtlich der Wahrnehmungs- und Vorstellungsleistung

Ein Vergleich des kleinsten Mittelwertes der Vorstellungsbedingung mit der Ratewahrscheinlichkeit von 50\% (T-Test mit einem Testwert von 50) ergibt ein signifikantes Ergebnis $(\mathrm{T}=3,45, \mathrm{df}=28, \mathrm{p}=.00)$. Das heißt, dass dieses Ergebnis und alle weiteren größer ausfallenden Ergebnisse vom Wert 50 verschieden sind und nicht mit Raten erklärt werden können. 
Die Varianzanalyse mit Messwiederholung ergab für den Faktor Erfahrung ein signifikantes Ergebnis $F(1,46)=5,78 ; p=.02 ; \eta^{2}=.11$.

Die deskriptive Statistik wird in der Tabelle 39 dargestellt.

Die durchschnittliche Wahrnehmungs- und Vorstellungsleistung beträgt ca. 65\% (siehe Tab. 46).

\begin{tabular}{|c|c|c|c|}
\hline \multirow{2}{*}{ Mittelwert } & Standardfehler & \multicolumn{2}{|c|}{$95 \%$ Konfidenzintervall } \\
\cline { 3 - 4 } & & Untergrenze & Obergrenze \\
\hline 64,75 & 1,22 & 62,29 & 67,20 \\
\hline
\end{tabular}

Tabelle 39: Gesamtmittel der Wahrnehmungs- und Vorstellungsleistung

Die Tabelle 40 stellt den Einfluss der Erfahrung (operationalisiert durch Gruppenunterschiede) auf die Wahrnehmungs- und Vorstellungsleistung dar. Dabei schneidet die Kontrollgruppe in der Wahrnehmungs- und Vorstellungsleistung durchschnittlich besser ab als die Versuchsgruppe (siehe auch Abb. 12).

\begin{tabular}{|c|c|c|c|c|}
\hline Gruppe & Mittelwert & Standardfehler & \multicolumn{2}{|c|}{$95 \%$ Konfidenzintervall } \\
\cline { 3 - 5 } & & & Untergrenze & Obergrenze \\
\hline VG & 61,82 & 1,80 & 58,20 & 65,43 \\
\hline KG & 67,67 & 1,65 & 64,36 & 70,98 \\
\hline
\end{tabular}

Tabelle 40: Mittelwerte und Standardfehler des Faktors Gruppe 


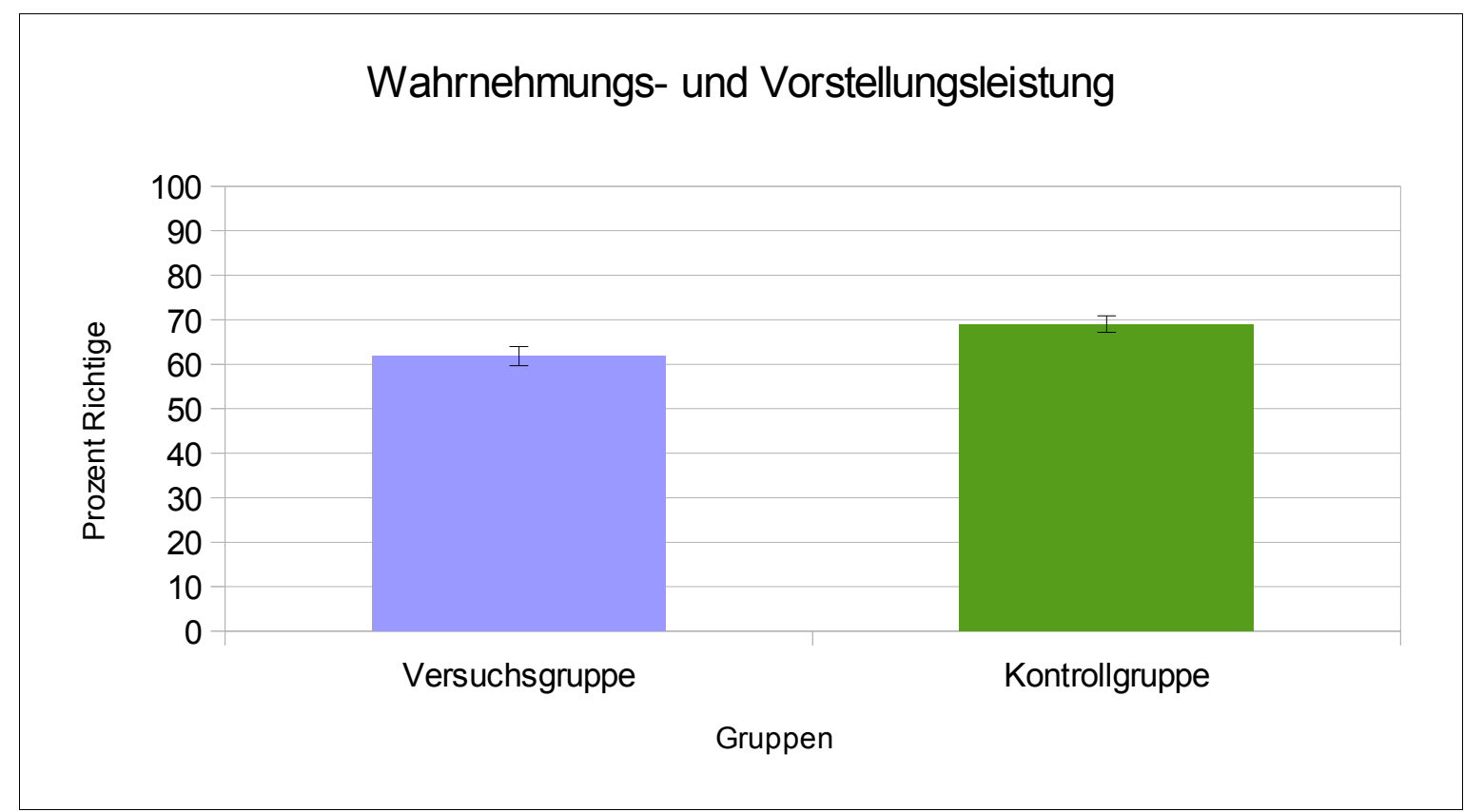

Abbildung 12: Ergebnisse der Varianzanalyse mit Messwiederholungen für die Wahrnehmungsund Vorstellungsleistung nach Gruppen

Der Versuch „50z" unterscheidet sich nur in der Kontrollgruppe von den beiden Versuchen Basisbedingung „0“ und „50g“ (siehe Tabelle 41). Die Werte im Versuch „50z“ fallen im Vergleich zum ersten Versuch „0“ und zum dritten Versuch „50g“ ab. Die Werte von Versuch „0“ und „50g“ sind ähnlich. In der Versuchsgruppe unterscheiden sich die drei Versuche Basisbedingung „0“, „50z“ und „50g“ nicht voneinander (siehe auch Abb. 13).

\begin{tabular}{|c|c|c|c|c|c|}
\hline \multirow{2}{*}{ Gruppe } & Bedingung & Mittelwert & Standardfehler & \multicolumn{2}{|c|}{ 95\% Konfidenzintervall } \\
\cline { 4 - 6 } & & & & Untergrenze & Obergrenze \\
\hline \multirow{2}{*}{ VG } & Basisbed. 0 & 66,57 & 2,85 & 60,84 & 72,29 \\
\cline { 2 - 6 } & $50 \mathrm{z}$ & 57,70 & 2,84 & 51,99 & 63,40 \\
\cline { 2 - 6 } & $50 \mathrm{~g}$ & 61,19 & 2,98 & 55,22 & 67,17 \\
\hline \multirow{2}{*}{ KG } & Basisbed. 0 & 71,40 & 2,61 & 66,16 & 76,64 \\
\cline { 2 - 6 } & $50 \mathrm{z}$ & 59,34 & 2,60 & 54,11 & 64,56 \\
\cline { 2 - 6 } & $50 \mathrm{~g}$ & 72,28 & 2,72 & 66,81 & 77,75 \\
\hline
\end{tabular}

Tabelle 41: Mittelwerte und Standardfehler der Faktoren Gruppe und Versuchsbedingung 


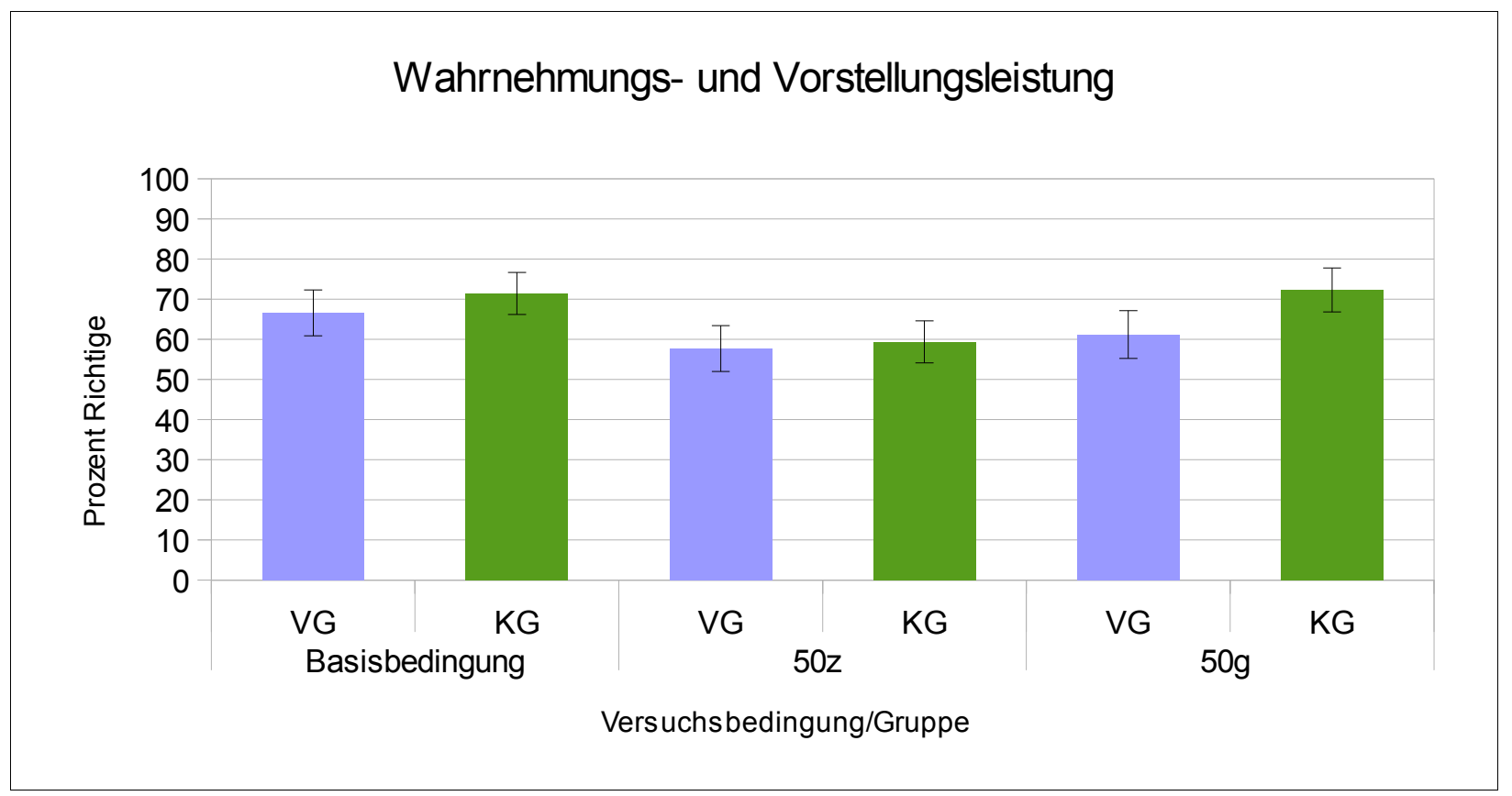

Abbildung 13: Interaktion von Gruppe und Versuchsbedingung hinsichtlich der Wahrnehmungs- und Vorstellungsleistung

\subsubsection{Diskussion}

Die Versuchsgruppe und die Kontrollgruppe unterscheiden sich hinsichtlich der Leistungen in den Wahrnehmungs- und Vorstellungsbedingungen signifikant, wobei die Kontrollgruppe bessere Vorstellungsleistungen erzielte (siehe Abb. 12). Dies kann nicht durch das Raten der Versuchspersonen erklärt werden.

Die Eingangshypothesen werden widerlegt. Die beiden Gruppen sind sowohl hinsichtlich der Wahrnehmungsleistungen als auch hinsichtlich der Vorstellungsleistungen vergleichbar.

Bedeutsame Unterschiede ergaben sich somit für die Vergleiche Basisbedingung „0 mit „50z“ sowie den Vergleich der Versuche „50z“ und „50g“ nur für die Kontrollgruppe (Abb. 13).

Demnach lässt sich folgendes Fazit ziehen:

Der Faktor Erfahrung hat einen Einfluss auf die Wahrnehmungs- und Vorstellungsleistung. Aber dieser Einfluss tritt nicht in der erwarteten Richtung auf. Die Annahme, dass Kinder, die über größere visuelle und motorische Erfahrung mit den Tennisbewegungen verfügen, in der Vorstellungsaufgabe besser abschneiden, konnte nicht bestätigt werden. Hingegen schnitten die Kinder der Kontrollgruppe im Vergleich 
zur Versuchsgruppe besser ab. Dies widerspricht den Studien von Calvo-Merino et al. $(2005,2006)$.

Die Befunde sprechen zunächst für die Hypothese von Ishai und Sagi (1997b). Demnach hätten die Kinder aus dem Langzeitgedächtnis Vorstellungen gebildet, die die Wahrnehmung (Bedingung „0“) stören.

Folgende Erklärungsmöglichkeiten bieten sich an:

\section{Rolle der Zeit für die Aktivierung des Bewegungswissens}

Dies könnte einerseits dadurch erklärt werden, dass die Tennis-Experten längere Zeit benötigen, um ihr Wissen zu aktivieren und für den Test zu nutzen. Gerade, da die „Experten“ hier Lernanfänger waren, scheint dies eine mögliche Erklärung zu sein. Bei visuellen Vorstellungen konnten dies Kosslyn et al. (1990) nachweisen. Hierbei waren 5Jährige den älteren Kindern unterlegen und brauchten bei allen Vorstellungsaufgaben mehr Zeit. Andererseits könnte es daran gelegen haben, dass die Kinder der Kontrollgruppe im Verlauf des Versuchs visuelle Lernerfahrungen erworben haben, die sie für die Beantwortung der Aufgabe einsetzten.

\section{Welche Rolle spielt das Wahrnehmungslernen?}

Reicht es aus, Bewegungen zu sehen und sie nicht auszuführen, um sie zu unterscheiden? Diese Frage bezieht sich auf das Wahrnehmungslernen. Dabei interessiert auch, wie oft sie hierfür präsentiert werden müssen im Vergleich zu dieser Studie: So zeigte Stränger (1977), dass das fünfmalige Sehen, wie ein mechanisches Puzzle gelöst wurde, ausreichte, um ähnlich gut abzuschneiden wie die Gruppe, die das Puzzle nach jeder Präsentation spielen und lösen konnte. Während Stränger (ebd.) eine Rückmeldung gab, ist eine solche hier in dieser Untersuchung nicht vorgesehen. Dennoch scheint es auszureichen, dass bei dem in dieser Dissertation verwendeten Versuchsablauf Wahrnehmungslernen stattfindet.

Kontrollgruppenkinder dieser Dissertation haben die Aufgabe also womöglich durch visuelles Lernen bewältigt. Dies wird durch den Befund gestärkt, dass die Prozentzahl richtig gelöster Aufgaben in der Basisbedingung „0“ ähnlich gut ist wie in der dritten Bedingung (,50g“) bzw. sich die Leistung von der Aufgabe „50z“ (zweite Aufgabe) zur Aufgabe „50g“ (dritte Aufgabe) steigert.

Demnach hat in diesem Versuch das Beobachtungslernen eine größere Bedeutung als die Bewegungserfahrung. 


\section{Rolle von Intentionen}

Eine dritte Erklärung könnte sein, dass beide Gruppen womöglich unterschiedliche Intentionen mit der Aufgabe verbunden haben. Hinweise hierzu gibt es von Decety et al. (1997). Sie fanden heraus, dass jeweils unterschiedliche Gehirnareale aktiv sind, je nachdem, ob eine Handlung mit der Intention nachgeahmt wird, sie anschließend zu imitieren oder sie nur wiederzuerkennen. Es könnte sein, dass die Tenniskinder die Präsentation der Videoaufnahmen mit der Intention verbinden, sie später nachzuahmen. Diese Intention war bei den Kontrollgruppenkindern nicht gegeben. In welcher Richtung sich dieser Effekt auswirkt, ist jedoch nicht bekannt.

\section{Rolle von körperlicher Aktivität}

Der „embodied cognition“-Ansatz sagt voraus, dass beispielsweise bei Erschöpfung die zeitliche Dauer einer vorgestellten im Vergleich zu einer tatsächlich ausgeführten Bewegung beeinflusst wird. Proffitt et al. (1995) zeigten, dass die Wahrnehmung von physikalischen Eigenschaften vom körperlichen Zustand (z.B. der Bewegungsfähigkeit oder dem Ermüdungszustand) abhängt. So wird ein Berg steiler wahrgenommen, wenn sich die Person auf dem Berg befindet oder wenn die Person zuvor einen Ausdauer-Lauf absolviert hatte.

Die Kinder der Versuchsgruppe machten vor und nach der Untersuchung Sport. Sie waren womöglich schon erschöpft. Deshalb könnte die mentale Simulation von Bewegungen länger angedauert haben und demzufolge verließen womöglich die Informationen schneller das Kurzzeitgedächtnis. 


\subsection{Studie 3: Einfluss des Geschlechts auf Wahrnehmung und Vorstellung von Bewegungen in der Kindheit}

In dieser Studie 3 ging es um den möglichen Einfluss des Geschlechts auf die Wahrnehmungs- und Vorstellungsleistung. Der Hypothese nach hat das Geschlecht einen Einfluss auf die Wahrnehmung und Vorstellung von Bewegungen, wobei Jungen bei den Vorstellungsaufgaben besser abschneiden sollten als Mädchen. Hingegen sollten sich Jungen und Mädchen nicht in der Basisbedingung „0“ unterscheiden.

\subsubsection{Stichprobe}

Die Stichprobe entsprach der in der Studie 2 dargestellten Charakteristik (siehe Kap. 8.4.1).

\subsubsection{Methode}

Instrumente, Untersuchungsablauf und Auswertungsprozedur waren wie bei Studie 2. Unterschiede gab es nur bezüglich der ausgewerteten Variablen: So ging das Geschlecht als between-subject-Faktor in die Berechnung der Varianzanalyse mit Messwiederholung ein.

\subsubsection{Ergebnisse der Studie 3}

Die Varianzanalyse mit Messwiederholung ergab für den Faktor Geschlecht ein signifikantes Ergebnis $F(1,46)=7,84 ; p=.01 ; \eta^{2}=.15$.

Die Tabelle 42 gibt die Ergebnisse der Varianzanalyse mit Messwiederholung bezüglich des Faktors Geschlechts wieder. Dabei schneiden Mädchen durchschnittlich besser ab als Jungen (siehe auch Abb. 14).

\begin{tabular}{|c|c|c|c|c|}
\hline \multirow{2}{*}{ Geschlecht } & Mittelwert & Standardfehler & \multicolumn{2}{|c|}{$95 \%$ Konfidenzintervall } \\
\cline { 3 - 5 } & & & Untergrenze & Obergrenze \\
\hline männlich & 61,34 & 1,68 & 57,97 & 64,71 \\
\hline weiblich & 68,15 & 1,77 & 64,60 & 71,71 \\
\hline
\end{tabular}

Tabelle 42: Mittelwerte und Standardfehler des Faktors Geschlecht 


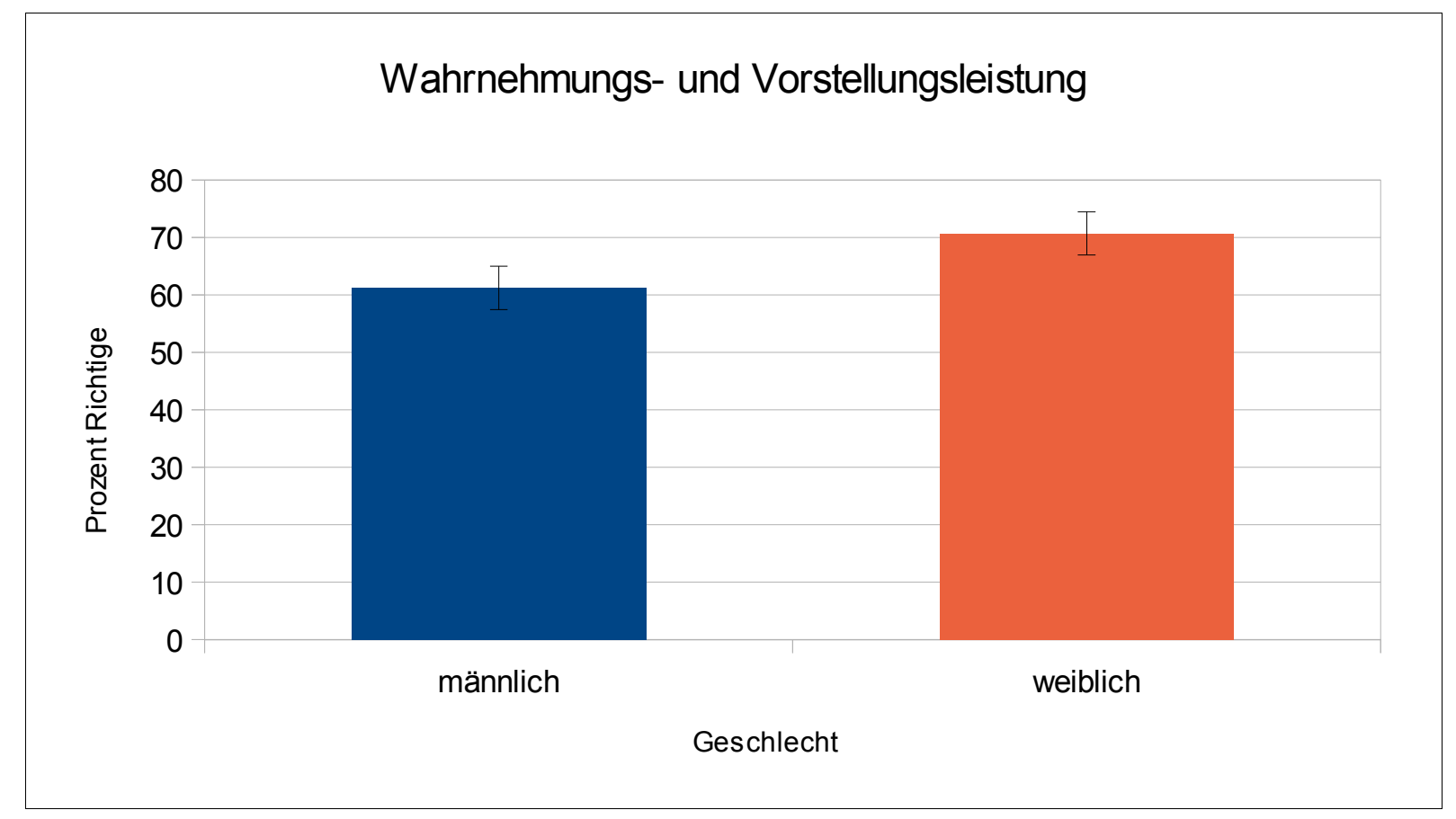

Abbildung 14: Ergebnisse der Varianzanalyse zum Faktor Geschlecht über alle Versuchsbedingungen

Die Interaktion zwischen den Faktoren Erfahrung und Geschlecht ergab ebenfalls ein signifikantes Ergebnis: $F(1,46)=8,55 ; p=.01, \eta^{2}=.16$.

Wie die Tabelle 43 zeigt, unterscheiden sich Jungen nicht in den Gruppen, die Mädchen der Kontrollgruppe schneiden besser ab als die Mädchen der Versuchsgruppe (vgl. auch Abb. 15).

\begin{tabular}{|c|c|c|c|c|c|}
\hline Gruppe & Geschlecht & Mittelwert & Standardfehler & \multicolumn{2}{|c|}{$95 \%$ Konfidenzintervall } \\
\cline { 4 - 6 } & & & & Untergrenze & Obergrenze \\
\hline \multirow{2}{*}{ VG } & männlich & 61,97 & 2,37 & 57,20 & 66,74 \\
\cline { 2 - 6 } & weiblich & 61,67 & 2,70 & 56,23 & 67,10 \\
\hline \multirow{2}{*}{ KG } & männlich & 60,70 & 2,37 & 55,94 & 65,47 \\
\cline { 2 - 6 } & weiblich & 74,64 & 2,28 & 70,05 & 79,24 \\
\hline
\end{tabular}

Tabelle 43: Mittelwerte und Standardfehler der Faktoren Gruppe und Geschlecht 


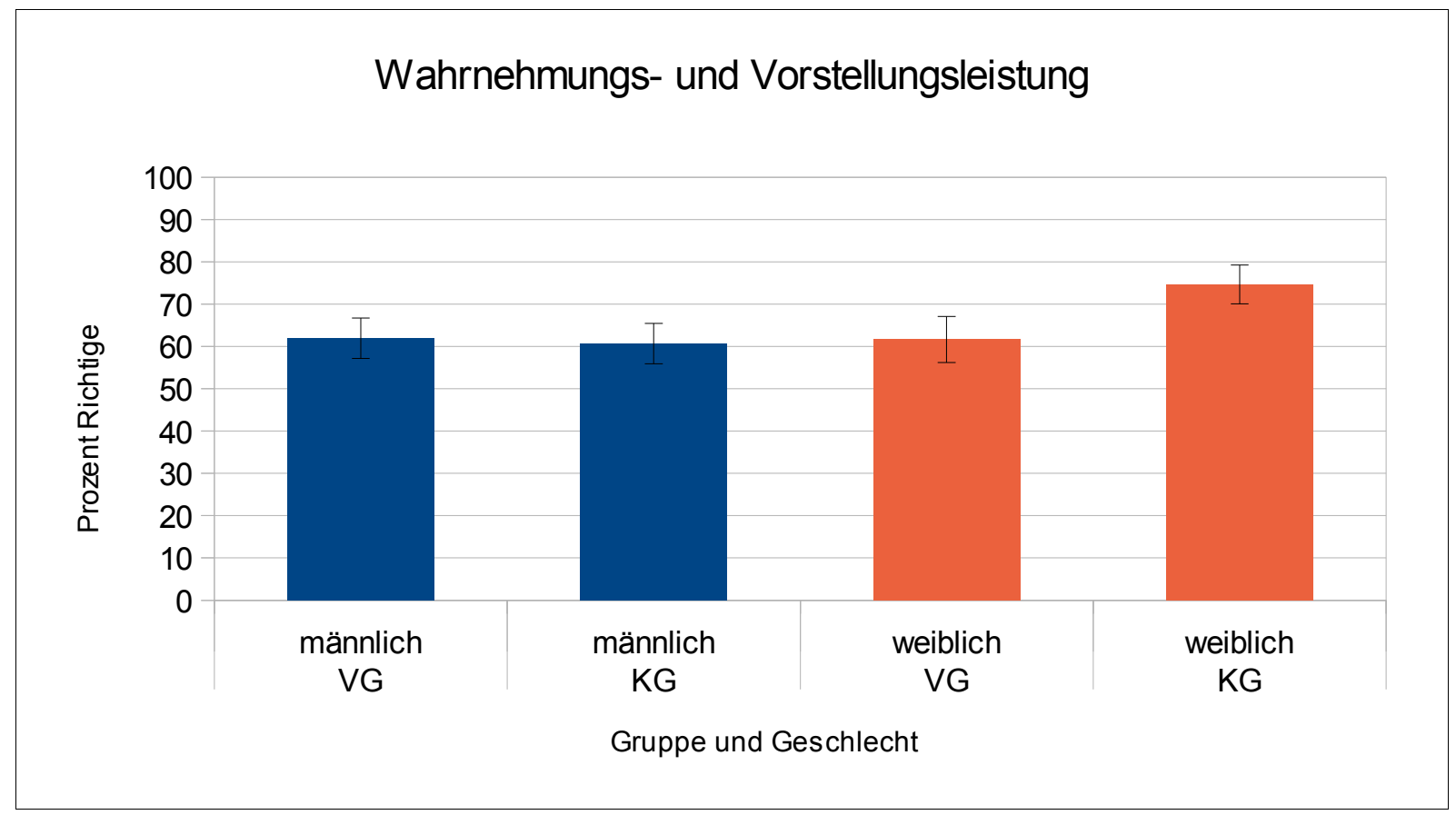

Abbildung 15: Ergebnisse der Varianzanalyse zum den Faktoren Gruppe und Geschlecht

\begin{tabular}{|c|c|c|c|c|c|}
\hline Geschlecht & Bedingung & Mittelwert & Standardfehler & \multicolumn{2}{|c|}{$95 \%$ Konfidenzintervall } \\
\cline { 4 - 6 } & & & & Untergrenze & Obergrenze \\
\hline \multirow{2}{*}{ männlich } & Basisbed. 0 & 66,66 & 2,65 & 61,32 & 72,00 \\
\cline { 2 - 6 } & $50 \mathrm{z}$ & 54,16 & 2,64 & 48,84 & 59,48 \\
\cline { 2 - 6 } & $50 \mathrm{~g}$ & 63,19 & 2,77 & 57,62 & 68,76 \\
\hline \multirow{2}{*}{ weiblich } & Basisbed. 0 & 71,31 & 2,80 & 65,67 & 76,94 \\
\cline { 2 - 6 } & $50 \mathrm{z}$ & 62,87 & 2,79 & 57,26 & 68,49 \\
\cline { 2 - 6 } & $50 \mathrm{~g}$ & 70,28 & 2,92 & 64,40 & 76,16 \\
\hline
\end{tabular}

Tabelle 44: Mittelwerte und Standardfehler der Faktoren Geschlecht und Versuchsbedingung

Wie in der Tabelle 44 und in Abbildung $16 \mathrm{zu}$ sehen ist, unterscheidet sich bei den Jungen nur der Versuch „50z" von der Basisbedingung „0“, die Werte hier sind geringer als in der Basisbedingung. Die Versuche „0“ und „50g“ unterscheiden sich nicht. Bei den Mädchen unterscheiden sich alle drei Versuche nicht voneinander. 


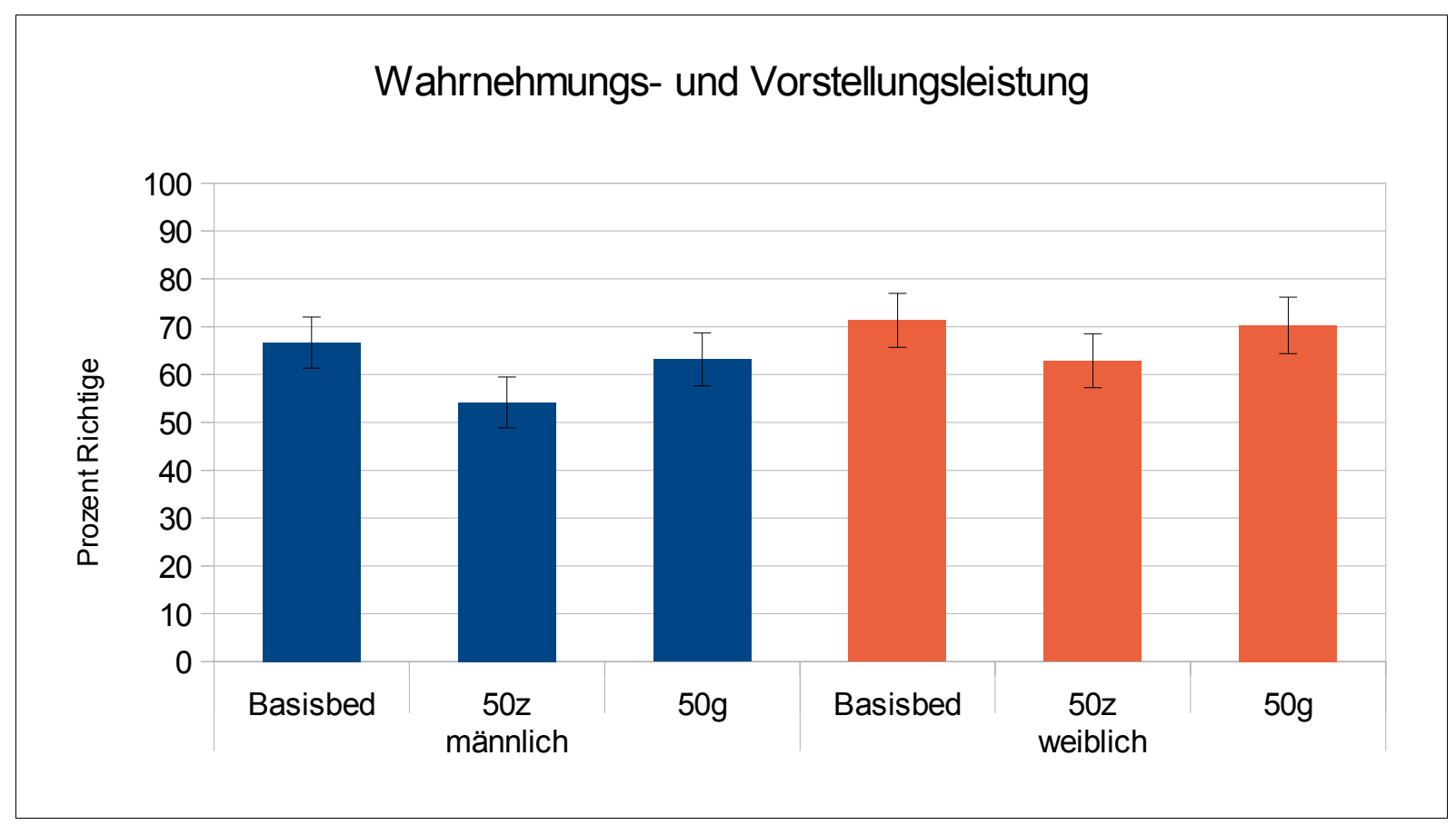

Abbildung 16: Ergebnisse der Varianzanalyse zur Wahrnehmungs- und Vorstellung bezüglich der Faktoren Versuchsbedingung und Geschlecht

\subsubsection{Diskussion}

Die Hypothese aus der Literatur, dass Jungen besser beim Sich-Vorstellen abschneiden als Mädchen, lässt sich zunächst nicht bestätigen. Die Hypothese, dass es keine Geschlechterunterschiede bei der Wahrnehmungsbedingung "0“ gibt, wird nicht widerlegt. Da beide Hypothesen durch ein logisches UND verbunden sind, gilt die Eingangshypothese als nicht bestätigt.

Im Ergebnis schneiden Mädchen signifikant besser ab als Jungen (Abb. 15). Insbesondere schneiden die Mädchen der Kontrollgruppe besser ab als die Mädchen der Versuchsgruppe (Abb. 16). Bei den Jungen unterscheidet sich die Wahrnehmungsbedingung "0“ von der ersten Vorstellungsbedingung "50z" signifikant. Bei Mädchen ergeben sich keine Unterschiede bezüglich der verschiedenen Versuchsbedingungen.

Jedoch wird hier davon ausgegangen, dass es Jungen schwerer haben, ihre dynamischen Vorstellungen zu bilden. Dabei ist es unerheblich, ob diese Vorstellungen aus dem Langzeitgedächtnis oder gerade im Moment der Aufgabe generiert werden. Dies widerspricht jedoch der Theorie von Ishai und Sagi (1997b).

Aufgrund der unterschiedlichen Geschlechterverteilung in den Gruppen können die Ergebnisse nur unter Berücksichtigung des Faktors Geschlecht dargestellt und 
interpretiert werden.

Es werden folgende Alternativerklärungen diskutiert:

\section{Erklärung 1: Strategieeinsatz}

Eine Erklärung für den Befund, dass Mädchen besser abschneiden als Jungen, bietet möglicherweise die Verwendung unterschiedlicher Strategien: Mädchen merken sich womöglich die Standbilder und gleichen sie ab, Jungen verwenden eine dynamische Strategie. Sie simulieren die nicht vorhandenen Bilder und ergänzen sie mental zu einem kompletten Bild. Dabei scheint letztere Strategie mehr Aufwand zu bedeuten und mehr Zeit zu beanspruchen. Hierfür spricht auch die geschlechtsspezifische Interaktion von Bedingung und Geschlecht (Abb. 16), so dass sich hier bei den Jungen die Bedingungen „0“ und „50z“ voneinander unterscheiden. Bei den Mädchen sind die Ergebnisse innerhalb der Versuchsbedingungen vergleichbar, bei den Jungen nicht.

Mädchen könnten demnach anders an die Aufgabe herangehen als Jungen. Der Einfluss der Gruppe auf die Leistungen der Mädchen könnte, wie in Kap. 8.4.4 beschrieben, auf die unterschiedliche körperliche Aktivität bei der Durchführung der Untersuchung zurückzuführen sein.

Wird nach Bedingung und Geschlecht unterschieden (Abb. 16), dann unterscheiden sich die Mädchen in den unterschiedlichen Versuchsbedingungen nicht voneinander. Jungen hingegen unterscheiden sich in den beiden ersten Bedingungen „0“ und „50z“. Dieser Befund deutet auf den Einfluss der Versuchsbedingung bei den Jungen, nicht so bei den Mädchen. Bei den Jungen gibt es hiernach quantifizierbare Unterschiede zwischen Wahrnehmungs- und Vorstellungsleistungen, während bei Mädchen in diesem Alter die Wahrnehmung ähnlich der Vorstellung abläuft.

Für den Befund, dass Mädchen der Kontrollgruppe im Versuch „50g“ signifikant besser als die Mädchen der Versuchsgruppe abschneiden, bieten sich zwei Erklärungen an:

\section{Erklärung 2: Motivation}

Die motivationale Komponente könnte eine Rolle spielen, unklar bleibt, wieso dann die Mädchen der Kontrollgruppe nicht über alle Versuche hinweg besser waren als die Versuchsgruppe und ausgerechnet im letzten Versuch besser abschnitten. Diese Erklärung ist demnach eher unwahrscheinlich.

\section{Erklärung 3: körperliche Verfassung}

Möglich ist auch der Faktor Erschöpfung, der zum Zeitpunkt des dritten Versuchs „50g“ 
besonders für die Unterschiede beigetragen haben könnte. Denn die Versuchsgruppe hatte womöglich in dieser Zeit ein besonders intensives Programm zu absolvieren, was die kognitive Leistung einschränkte. Dennoch bleibt unklar, wieso nicht auch bei den Jungen solche Unterschiede zur Versuchsgruppe im letzten Versuch „50g“ aufgezeigt werden konnten. Dies bedarf weiterer Forschung. 


\subsection{Studie 4: Einfluss der Versuchsbedingung auf Wahrnehmung und Vorstellung von Bewegungen in der Kindheit}

In dieser Studie wurde der Einfluss der Versuchsbedingung auf die Wahrnehmung und Vorstellung von Bewegungen untersucht. Dies diente dazu, quantitative Vergleiche zwischen Wahrnehmungs- und Vorstellungsleistungen durchzuführen. Die beiden Vorstellungsbedingungen „50z" und "50g" sollten sich von der Wahrnehmungsbedingung „0“ unterscheiden.

\subsubsection{Stichprobe}

Die Stichprobe entsprach der in der Studie 2 dargestellten Stichprobe (siehe Kap. 8.4.1).

\subsubsection{Methode}

In der Studie 4 ging es um den möglichen Einfluss der Versuchsbedingung auf die Wahrnehmungs- und Vorstellungsleistung. Instrumente, Untersuchungsablauf und Auswertungsprozedur waren mit kleinen Unterschieden identisch mit der Studie 2. Diese Unterschiede standen in Verbindung mit der untersuchten Variablen Versuchsbedingung. Dabei ging die Versuchsbedingung als within-subject-Faktor in die Berechnung der Varianzanalyse mit Messwiederholung ein.

\subsubsection{Ergebnisse der Studie 4}

Die Tabelle 45 gibt die Ergebnisse der Varianzanalyse mit Messwiederholung bezüglich des Messwiederholungsfaktors Versuchsbedingung wieder.

Auch die Versuchsbedingungen (Messwiederholungsfaktor) unterscheiden sich im Test bedeutsam voneinander: Wilks-Lambda $(2,45)=7.02 ; p<.01 ; \eta^{2}=.24$. Im F-Test wird der Messwiederholungsfaktor ebenfalls signifikant: $F(1,46)=2827,27 ; p<.01 ; \eta^{2}=.98$.

Dabei zeigt sich in Tabelle 45, dass sich die Basisbedingung "0“ signifikant von der Bedingung „50" unterscheidet, insofern als dass die Vorstellungsleistung in diesem Versuch geringer ausfällt als die Wahrnehmungsleistung. Auch unterscheidet sich die Bedingung „50z" von der Bedingung „50g“ signifikant. Die Werte der Bedingung „50g“ fallen dabei höher aus. Allerdings weisen die Versuche Basisbedingung „0“ und „50g“ keine Unterschiede auf, die Werte in den Versuchen „0“ und „50g“ sind demnach ähnlich. 


\begin{tabular}{|c|c|c|c|c|}
\hline Bedingung & Mittelwert & \multirow{2}{*}{ Standardfehler } & \multicolumn{2}{|c|}{$95 \%$ Konfidenzintervall } \\
\cline { 3 - 5 } & & & Untergrenze & Obergrenze \\
\hline Basisbed. 0 & 68,98 & 1,93 & 65,10 & 72,87 \\
\hline $50 \mathrm{z}$ & 58,52 & 1,92 & 54,65 & 62,39 \\
\hline $50 \mathrm{~g}$ & 66,74 & 2,01 & 62,69 & 70,78 \\
\hline
\end{tabular}

Tabelle 45: Mittelwerte und Standardfehler des Messwiederholungsfaktors Versuchsbedingung

Die Ergebnisse dieser Varianzanalyse mit Messwiederholungen werden in der Abb. 17 dargestellt.

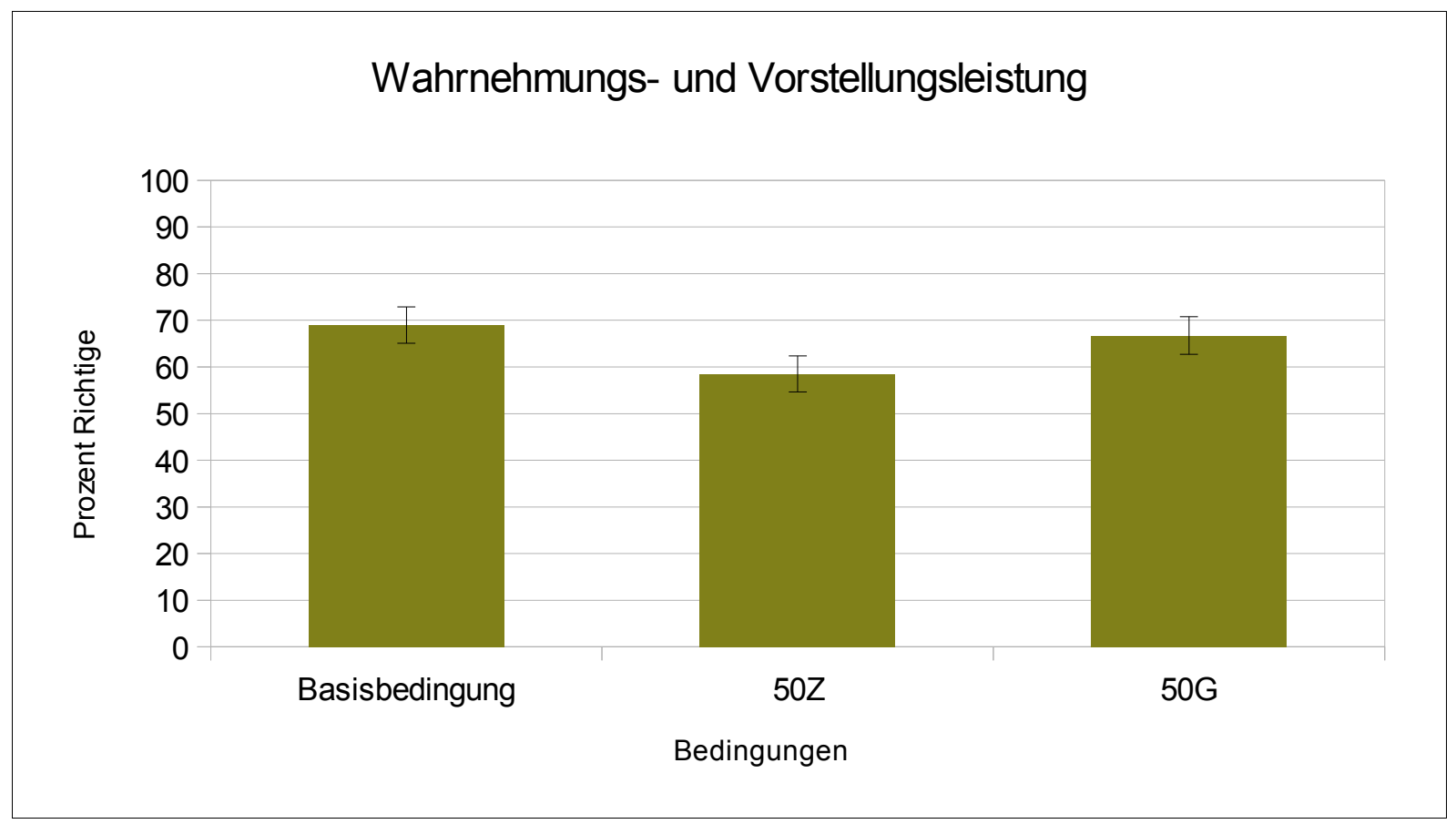

Abbildung 17: Ergebnisse der Varianzanalyse mit Messwiederholungen für die Wahrnehmungs- und Vorstellungsleistung nach Versuchsbedingungen

\subsubsection{Diskussion}

Die Hypothese, dass sich die beiden Vorstellungsbedingungen von der Wahrnehmungsbedingung unterscheiden, ist teilweise bestätigt worden. Die Versuchsbedingung "50z" unterscheidet sich zwar signifikant von der Wahrnehmungsbedingung "0“. Aber es gibt keine Unterschiede zwischen der Wahrnehmungsbedingung "0" und der Vorstellungsbedingung „50g“ (siehe Abb. 17). Dies gilt, obwohl sich der Versuch „50z" von der Basisbedingung „0“ im Versuchsaufbau in der Form unterscheidet, dass er abgedeckte Flächen enthält. 
Es können weiterhin folgende Unterschiede zwischen Wahrnehmung und Vorstellungen aufgezeigt werden:

1) Die Wahrnehmungsleistung ist ohne Abdeckung im Mittel bei 68,98 Prozent richtiger Antworten. Hingegen verschlechtert sich die Leistungen auf 58,52 Prozent richtiger Antworten beim Test mit der Hälfte abgedeckter Bereiche („50z“), wobei dieser Unterschied signifikant ist (siehe Abb. 17).

2) Wird das Ergebnis des Tests mit 50\% unzusammenhängend abgedeckter Fläche hinzugezogen (,50g“), dann ergibt sich nochmalig eine Steigerung der Leistung auf 66,74 Prozent richtiger Antworten, was eine signifikante Verbesserung zum Test „50z“ darstellt. Gleichzeitig unterscheiden sich die Teilversuche „0“ und „50g“ nicht signifikant voneinander.

Diese beiden Ergebnisse werden als Hinweis auf die mentale Ergänzungsleistung interpretiert. 


\subsection{Studie 5: Einfluss von Trainingserfahrung auf Wahrnehmungen und Vorstellung von Bewegungen in der Versuchsgruppe}

Es wurde der Einfluss der unterschiedlichen Trainingserfahrung auf die Wahrnehmungsund Vorstellungsleistung ermittelt. Damit sollte festgestellt werden, ob beide Teile der Versuchsgruppe miteinander vergleichbar waren und zusammengefasst werden konnten. Jede der beiden unterschiedlichen Trainingsgruppen der Versuchsgruppe wurde altersgemäß trainiert. Den Hypothesen nach sollten beide Gruppen vergleichbar sein.

\subsubsection{Stichprobe}

Die Stichprobe entspricht der in der Studie 1 (siehe Kap. 8.3.1).

\subsubsection{Methode}

Anhand des Altersmittels wurde die Versuchsgruppe geteilt, was den zwei unterschiedlich beschulten Gruppen innerhalb der Versuchsgruppe entsprach. Es wurde eine Varianzanalyse mit den beiden unterschiedlich trainierten Gruppen als Gruppenfaktor berechnet.

\subsubsection{Ergebnisse der Studie 5}

In der Tabelle 46 werden die Ergebnisse der deskriptiven Statistik gezeigt. 


\begin{tabular}{|c|c|c|c|c|}
\hline & Gruppe-VG & $\begin{array}{l}\text { Mittel- } \\
\text { wert }\end{array}$ & $\begin{array}{c}\text { Standard- } \\
\text { abweichung }\end{array}$ & $\mathrm{N}$ \\
\hline \multirow[t]{3}{*}{ Basisbed. 0} & 1 & 73,32 & 18,07 & 5 \\
\hline & 2 & 66,70 & 0,00 & 2 \\
\hline & Gesamt & 71,43 & 15,11 & 7 \\
\hline \multirow[t]{3}{*}{$50 Z$} & 1 & 58,34 & 13,17 & 5 \\
\hline & 2 & 54,15 & 5,87 & 2 \\
\hline & Gesamt & 57,14 & 11,21 & 7 \\
\hline \multirow[t]{3}{*}{$50 \mathrm{G}$} & 1 & 64,18 & 3,76 & 5 \\
\hline & 2 & 66,70 & 0,00 & 2 \\
\hline & Gesamt & 64,90 & 3,30 & 7 \\
\hline \multirow[t]{3}{*}{25} & 1 & 63,32 & 12,63 & 5 \\
\hline & 2 & 62,50 & 5,94 & 2 \\
\hline & Gesamt & 63,09 & 10,60 & 7 \\
\hline \multirow[t]{3}{*}{$33 Z$} & 1 & 75,00 & 17,68 & 5 \\
\hline & 2 & 66,65 & 23,55 & 2 \\
\hline & Gesamt & 72,61 & 17,81 & 7 \\
\hline \multirow[t]{3}{*}{$33 G$} & 1 & 64,98 & 9,15 & 5 \\
\hline & 2 & 75,00 & 11,74 & 2 \\
\hline & Gesamt & 67,84 & 10,13 & 7 \\
\hline \multirow[t]{3}{*}{$67 Z$} & 1 & 64,98 & 19,90 & 5 \\
\hline & 2 & 70,85 & 5,87 & 2 \\
\hline & Gesamt & 66,66 & 16,68 & 7 \\
\hline \multirow[t]{3}{*}{ 67G } & 1 & 70,00 & 9,49 & 5 \\
\hline & 2 & 65,65 & 22,13 & 2 \\
\hline & Gesamt & 68,76 & 12,09 & 7 \\
\hline
\end{tabular}

Tabelle 46: Ergebnisse der deskriptiven Statistik (Anmerkung: jüngere Kinder (1), ältere Kinder (2))

Es wurde ein Levene-Test durchgeführt (siehe Tab. 47). Dieser prüft, ob die Fehlervarianz der abhängigen Variablen über die Gruppen hinweg gleich ist (Nullhypothese). Der Levene-Test ergibt für den Teilversuch „67g“ ein signifikantes Ergebnis (rot markiert). Es kann deshalb nicht von der Erfüllung der Voraussetzungen für die Varianzanalyse ausgegangen werden. Eine Alternative wäre, hier ein verteilungsfreies Verfahren einzusetzen (Bortz, 1993). 


\begin{tabular}{|l|r|c|}
\hline & \multicolumn{1}{|c|}{$\mathrm{F}$} & Signifikanz \\
\hline Basisbed. 0 &, 274 & .62 \\
\hline $50 Z$ & 4,544 & .09 \\
\hline $50 \mathrm{G}$ & 4,700 & .08 \\
\hline 25 & 1,887 & .23 \\
\hline $33 Z$ &, 024 & .88 \\
\hline $33 \mathrm{G}$ &, 235 & .65 \\
\hline $67 Z$ & 2,819 & .15 \\
\hline $67 G$ & 21,545 & .01 \\
\hline
\end{tabular}

Tabelle 47: Ergebnisse des Levene-Tests (Anmerkung df Zähler=1 df Nenner=5, N=7)

Der multivariate Test ergab für den Gruppenfaktor (Gruppe-VG) keinen signifikanten Effekt $F(4,1)=.09 ; p=.97$. Die Ergebnisse des Signifikanztests werden in der folgenden Tabelle 48 gezeigt. Es ergeben sich keine signifikanten Ergebnisse hinsichtlich des Faktors Erfahrung.

\begin{tabular}{|l|r|c|}
\hline Abhängige Variable & \multicolumn{1}{|c|}{$F$} & Signifikanz \\
\hline Basisbed. 0 &, 565 & .49 \\
\hline $50 Z$ &, 075 & .80 \\
\hline $50 \mathrm{G}$ &, 469 & .53 \\
\hline 25 &, 019 & .90 \\
\hline $33 Z$ &, 311 & .61 \\
\hline $33 \mathrm{G}$ & 1,135 & .35 \\
\hline $67 Z$ &, 163 & .71 \\
\hline $67 G$ &, 070 & .80 \\
\hline
\end{tabular}

Tabelle 48: Ergebnisse der Signifikanztests hinsichtlich des Erfahrungsfaktors zu den Wahrnehmungs- und Vorstellungsleistungen

\subsubsection{Diskussion}

Obwohl die Versuchsgruppe unterschiedlich beschult wurde, wirkte sich dies nicht auf die Wahrnehmungs- und Vorstellungsleistung aus. Die jüngeren Kinder schnitten in der Vorstellungsbedingung gleich gut ab wie die älteren Kinder.

Dennoch gilt Vorsicht bei der Interpretation, denn die Voraussetzungen für die Varianzanalyse sind bei einem Teilversuch nicht gegeben. Außerdem sind die Fallzahlen sehr klein. 


\subsection{Studie 6: Einfluss von Trainingserfahrung auf Bewegungslernen in der Versuchsgruppe}

Der Einfluss des unterschiedlichen Trainings in der Versuchsgruppe wurde hier auf das Bewegungslernen untersucht. Die Tenniskinder trainierten in zwei verschiedenen Gruppen, die jeweils entsprechend ihres Alters trainiert wurden. Der Hypothese nach sollten beide Gruppen innerhalb der Versuchsgruppe vergleichbar sein.

\subsubsection{Stichprobe}

Die Stichprobe entsprach der in der Studie 1 (siehe Kap. 8.3.1) dargestellten Stichprobe.

\subsubsection{Methode}

Um zu prüfen, inwieweit es Einflüsse des Trainings in den 2 Trainingsgruppen der Versuchsgruppe auf das Bewegungslernen gab, wurde die Versuchsgruppe anhand des Altersmittels geteilt (Variable Gruppe_VG). Dies entsprach der Aufteilung der Versuchsgruppe $\mathrm{zu}$ den unterschiedlichen, jeweils altersgerecht beschulten Versuchsgruppenteilen.

Bewegungslernen wurde hierbei als Veränderungen des Bewegungslernens aufgefasst und mittels Differenzbildung des Bewegungslernens (Gesamtwert über alle Skalen) zum Zeitpunkt 3 und des Bewegungslernens (Gesamtwert über alle Skalen) zum Zeitpunkt 2 gebildet (MZP2-MZP3=MIN2_3).

Damit ergaben sich bei geringeren Werten auch geringere Beherrschungsgrade der Tennistechnik. Dies sollte auf ein geringeres Bewegungslernen hinweisen.

Dabei wurde das Bewegungslernen durch das Ergebnis des Expertenratings mittels des „Fragebogens zur Beherrschung der Tennistechnik“ zu den jeweiligen Messzeitpunkten operationalisiert.

Folgende Eingangsvoraussetzungen der Tenniskinder wurden gemessen:

Erstens wurde die Anzahl der Sportarten mittels Bewegungstagebuch (Versuchsgruppe) ermittelt.

Zweitens wurden die Kinder zu Beginn der wissenschaftlichen Begleitung des Projekts "Jüngsten-Tennis“ hinsichtlich ihrer motorischen Gewandtheit untersucht (Zeitpunkt der Untersuchung: 25.6.2005). Diese wurde anhand des Kasten-Bumerang-Laufs ermittelt. Dieser Test berücksichtigt neben der Gewandtheit auch die Antizipationsfähigkeit (Beck 
\& Bös, 1995; Bös, 2001). Die Objektivität des Tests liegt nach Angaben von Beck und Bös (1995) bei durchschnittlich .96, die Reliabilität bei durchschnittlich .86. Der Test wurde auch validiert (siehe Gütekriterien vom AST in Bös und Wohlmann, 1987). Er ist normiert für eine Altersgruppe von 6 bis 18 Jahren. Dies stellte ein Problem dar, da 3 von 32 Kindern unter 6 Jahren waren. Es wurden für diese Kinder die Normen von der untersten Altersklasse verwendet, damit die Fallzahlen nicht noch geringer ausfielen. Außerdem ist kritisch zu bewerten, dass der Test keine Vorschriften für die Behandlung von Fehlern der Kinder vorsieht.

Beim Kasten-Bumerang-Lauf, einem Hindernislauf, bei dem es auf das Tempo ankommt, wird zu Beginn eine Rolle vorwärts ausgeführt und danach ein in der Mitte liegender Medizinball umlaufen, um zu einem der äußeren Kästen zu gelangen. Dieser Kasten wird erst übersprungen, dann durchkrochen, um wieder zum Medizinball zu gelangen. Insgesamt muss ein Kastenteil drei Mal übersprungen, durchkrochen und dazwischen der Medizinball umlaufen werden, bis das Kind wieder zur Startlinie gelangt. Die Kästen sind jeweils 2,5 Meter vom Medizinball entfernt, so auch der Startpunkt.

Es wurde drittens ein Bewegungstagebuch entwickelt (siehe Anhang 9.1), womit die Anzahl der ausgeübten Sportarten sowie die Verteilung von bewegungsnahen und bewegungsfernen Aktivitäten über den Tag erfasst werden konnten. Daraus wurde das Verhältnis von bewegungsnahen zu bewegungsfernen Aktivitäten ermittelt. Unter bewegungsnahen Aktivitäten wurden beispielsweise verstanden: Inlineskaten, Radfahren, Schwimmen, Turnen, Tanzen und sonstige bewegungsintensive Aktivitäten. Als bewegungsferne Aktivitäten wurde zum Beispiel abgefragt: Hausaufgaben machen, Lernen, am PC sitzen und sonstiges. Das Bewegungstagebuch wurde im September 2005 an die Eltern der Kinder des Projekts „Jüngsten-Tennis“ verteilt. Der Bewegungsalltag wurde über jeweils zwei Wochen protokolliert.

Eine letzte Kovariate für die spätere Analyse ist die Trainingsteilnahme. Die Teilnahme am Training wurde über einen Zeitraum von 28 Wochen (ohne Schulferien) gemessen. Hierzu diente eine Aufzeichnung des Tennistrainers als Hilfsmittel.

\subsubsection{Ergebnisse der Studie 6}

Bei der Variablen Trainingserfahrung wurde die Versuchsgruppe in zwei gleich große Hälften geteilt und ging als fester Faktor in die Analyse ein, da diese den zwei verschiedenen Trainingsgruppen entsprach. Es wurde eine Kovarianzanalyse der Variable Trainingserfahrung mit folgenden Kovariaten durchgeführt: Anzahl der 
(ausgeübten) Sportarten, Verhältnis der bewegungsnahen zu bewegungsfernen Aktivitäten sowie die Trainingstage in Prozent. Die abhängige Variable war hierbei die Differenz des Bewegungslernens von MZP2 und MZP3 (MIN2_3).

Die Tabelle 49 zeigt die deskriptive Statistik des Faktors Trainingserfahrung zu dieser Berechnung. Die Variable Trainingserfahrung wird nicht signifikant $(F(1,14)=0,05, p=.83$, $\left.\eta^{2}=.01\right)$. Das heißt, es bestehen keine überzufälligen Unterschiede zwischen jüngeren und älteren Kindern der Versuchsgruppe bezüglich des Bewegungslernens (Gesamtskala) (siehe Abb. 18).

\begin{tabular}{|c|c|c|c|}
\hline Versuchsgruppe & Mittelwert & $\begin{array}{c}\text { Standard- } \\
\text { abweichung }\end{array}$ & $\mathrm{N}$ \\
\hline jünger & 0.25 & 0.28 & 7 \\
\hline älter & 0.36 & 0.53 & 7 \\
\hline Gesamt & 0.30 & 0.41 & 14 \\
\hline
\end{tabular}

Tabelle 49: Mittelwerte und Standardabweichungen des Faktors Trainingserfahrung für Veränderungen im Bewegungslernen (Gesamtskala)

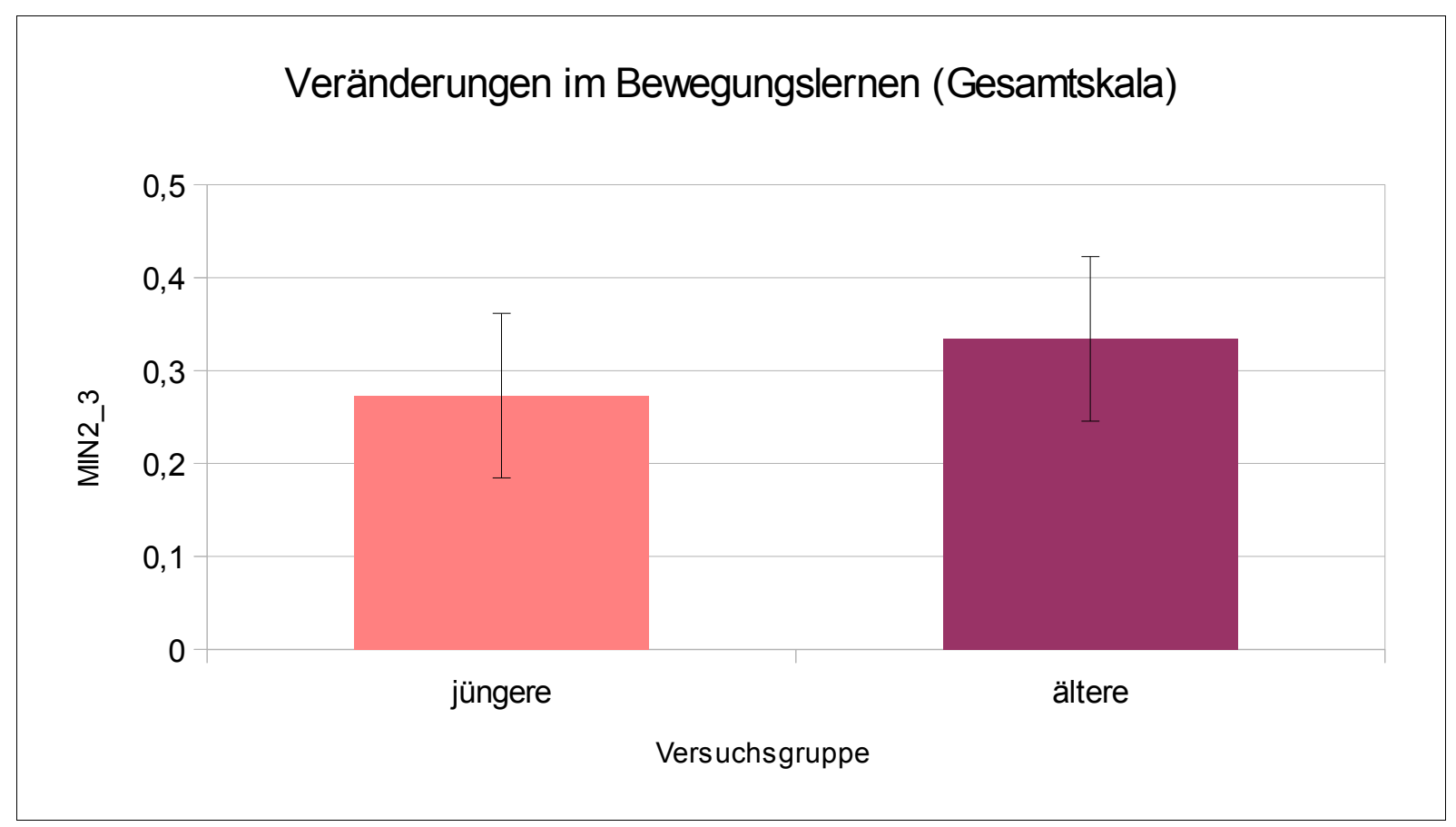

Abbildung 18: Ergebnis der Kovarianzanalyse zum Faktor Trainingserfahrung und dessen Einfluss auf das Bewegungslernen (Gesamtskala) (Jüngere und ältere Kinder wurden in den zwei verschiedenen Trainingsgruppen jeweils unterschiedlich beschult.)

Die Ergebnisse der Auswertung für die Kovariaten werden hier dargestellt: Die durchschnittliche Trainingsteilnahme betrug $75,4 \%$ (die Standardabweichung betrug 
12,89\%). Dies entspricht 21,1 Wochen. Die Trainingsteilnahme wird in Tabelle 50 dargestellt.

\begin{tabular}{|c|c|c|}
\hline \multicolumn{3}{|c|}{ Teilnahme am Training } \\
\hline $\begin{array}{c}\text { Trainings- } \\
\text { teilnahme } \\
\text { in \% }\end{array}$ & $\begin{array}{c}\text { Anzahl } \\
\text { der } \\
\text { Kinder }\end{array}$ & $\begin{array}{c}\% \\
\text { der } \\
\text { Kinder }\end{array}$ \\
\hline 54 & 1 & 3,6 \\
\hline 57 & 1 & 3,6 \\
\hline 61 & 7 & 25 \\
\hline 67 & 1 & 3,6 \\
\hline 71 & 1 & 3,6 \\
\hline 75 & 3 & 10,7 \\
\hline 79 & 3 & 10,7 \\
\hline 80 & 1 & 3,6 \\
\hline 82 & 1 & 3,6 \\
\hline 86 & 1 & 3,6 \\
\hline 89 & 5 & 17,9 \\
\hline 92 & 1 & 3,6 \\
\hline 93 & 1 & 3,6 \\
\hline 96 & 1 & 3,6 \\
\hline Gesamt & $\mathbf{2 8}$ & $\mathbf{1 0 0}$ \\
\hline
\end{tabular}

Tabelle 50: Trainingsteilnahme und Anteile

Die Ergebnisse des Bewegungstagebuchs (Versuchsgruppe) bzw. der Befragung (Kontrollgruppe) werden im Folgenden dargestellt:

Die Rücklaufquote des Bewegungstagebuchs betrug ca. $80 \%$.

Die Anzahl der ausgeführten Sportarten betrug bei der Versuchsgruppe (Kinder mit Tennistraining) insgesamt durchschnittlich 2,84 $(S D=1,2)$. Beispiele für häufig ausgeübte Sportarten neben dem Tennis waren Fußball, Turnen, Schwimmen oder Ballett. Die Ergebnisse des Bewegungstagebuchs werden in der Tabelle 51 dargestellt. 


\begin{tabular}{|c|c|c|c|}
\hline \multicolumn{4}{|c|}{ Art der Bewegungsaktivität } \\
\hline & bewegungsnah & bewegungsfern & Verhältnis \\
\hline AB & 1535 & 645 & 2,38 \\
\hline BL & 1580 & 740 & 2,14 \\
\hline DH & 1105 & 1615 & 0,68 \\
\hline FB & 2295 & 2535 & 0,91 \\
\hline GK & 1815 & 1325 & 1,37 \\
\hline HW & 1740 & 1500 & 1,16 \\
\hline IH & 3480 & 1785 & 1,95 \\
\hline JBR & 3045 & 1295 & 2,35 \\
\hline JB & 1430 & 475 & 3,01 \\
\hline JW & 1285 & 2305 & 0,56 \\
\hline JK & 1545 & 1045 & 1,48 \\
\hline KS & 1620 & 2560 & 0,63 \\
\hline IH & 1520 & 1775 & 0,86 \\
\hline LW & 1880 & 2820 & 0,67 \\
\hline MAS & 2545 & 935 & 2,72 \\
\hline MS & 2065 & 1730 & 1,19 \\
\hline MW & 1815 & 1050 & 1,73 \\
\hline MW & 3200 & 905 & 3,54 \\
\hline NM & 1085 & 1265 & 0,86 \\
\hline PH & 1310 & 1755 & 0,75 \\
\hline SK & 2100 & 2210 & 0,95 \\
\hline SP & 1675 & 1750 & 0,96 \\
\hline TB & 2670 & 1445 & 1,85 \\
\hline TH & 3980 & 2035 & 1,96 \\
\hline Mittel & 2013 & 1563 & 1,53 \\
\hline
\end{tabular}

Tabelle 51: bewegungsnahe und bewegungsferne Aktivtiäten bei den Kindern des Projekts „Jüngsten-Tennis“ (in Minuten), Verhältnis von bewegungsnahen zu bewegungsfernen Aktivitäten

Aus folgender Tabelle 52 ergibt sich die Anzahl der ausgeübten Sportarten nach Gruppen aufgeschlossen: 


\begin{tabular}{|l|c|c|c|c|c|c|}
\hline \multicolumn{5}{|c|}{ Anzahl der Sportarten nach Gruppen und in der Gesamtgruppe } \\
\hline $\begin{array}{l}\text { Anzahl der } \\
\text { Sportarten }\end{array}$ & \multicolumn{2}{|c|}{ VG } & \multicolumn{2}{c|}{ KG } & \multicolumn{2}{c|}{ Gesamtgruppe } \\
\hline & $\begin{array}{c}\text { Anzahl } \\
\text { der Kinder }\end{array}$ & $\begin{array}{c}\text { K der Kinder } \\
\text { der Kinder }\end{array}$ & $\begin{array}{c}\text { Anzahl } \\
\text { \% der Kinder }\end{array}$ & $\begin{array}{c}\text { Anzahl der } \\
\text { Kinder }\end{array}$ & $\begin{array}{c}\% \\
\text { der Kinder }\end{array}$ \\
\hline 0 & & & 5 & 18,5 & 5 & 10,2 \\
\hline 1 & & & 11 & 40,7 & 11 & 22,4 \\
\hline 2 & 11 & 50 & 6 & 22,2 & 17 & 34,7 \\
\hline 3 & 7 & 31,8 & 5 & 18,5 & 12 & 24,5 \\
\hline 4 & 2 & 9,1 & & & 2 & 4,1 \\
\hline 5 & 2 & 9,1 & & & 2 & 4,1 \\
\hline Gesamt & $\mathbf{2 2}$ & $\mathbf{1 0 0}$ & $\mathbf{2 7}$ & $\mathbf{1 0 0}$ & $\mathbf{4 9}$ & $\mathbf{1 0 0}$ \\
\hline
\end{tabular}

Tabelle 52: Häufigkeitsstatistik für die Anzahl der Sportarten

Der Chi-Quadrat-Test gibt an, ob sich beide Gruppen hinsichtlich der Anzahl der Sportarten unterscheiden. Er ergab diese Ergebnisse: Chi-Quadrat=22,89; df=5; $p<.01$. Das heißt, die Anzahl der Sportarten unterscheidet sich in den Gruppen bedeutsam.

Die mittlere Dauer von bewegungsnahen Aktivitäten betrug 2013 min (SD=771 min) und von bewegungsfernen Aktivitäten $1563 \min$ ( $S D=633$ min). Der Unterschied war signifikant $(T=2,295, \quad d f=23, \quad p=.031)$. Das mittlere Verhältnis zwischen bewegungsaktiven und bewegungsarmen Aktivitäten war größer als 1 und betrug 1,53 $(S D=0,83)$. Während ein Minimum von 1085 min bei bewegungsnahen Abläufen und von 475 min bei bewegungsfernen Aktivitäten ermittelt wurde, liegen die Maxima bei 3980 min versus 1820 min. In der Gruppe „Jüngsten-Tennis“ besteht mehrheitlich (konkret bei 14 von 24 Kindern) ein Verhältnis größer als 1 von bewegungsnahen zu bewegungsfernen Aktivitäten. Sie bewegen sich häufiger am Tag als dass sie z.B. fernsehen.

Nun werden die Ergebnisse des Kasten-Bumerang-Laufs veranschaulicht (siehe Tab. 53). Die Ergebnisse des Kasten-Bumerang-Tests waren mit einer Punktzahl von 2,7 Punkten überdurchschnittlich. 


\begin{tabular}{|c|c|c|c|c|}
\hline \multicolumn{5}{|c|}{ Kasten-Bumerang-Lauf } \\
\hline $\begin{array}{c}\text { Kind } \\
\text { (kodiert) }\end{array}$ & Alter & $\begin{array}{l}\text { Kasten-Bumerang- } \\
\text { Lauf (in Sekunden) }\end{array}$ & Fehler & Punkte \\
\hline$A B$ & 7 & 18,40 & & 4 \\
\hline $\mathrm{BL}$ & 5 & 17,90 & & 3 \\
\hline BT & 6 & 22,40 & & 2 \\
\hline $\mathrm{DH}$ & 9 & 16,60 & & 3 \\
\hline FB & 9 & 17,60 & & 3 \\
\hline $\mathrm{FL}$ & 9 & 15,40 & & 4 \\
\hline GK & 6 & 18,60 & FF & 3 \\
\hline $\mathrm{HB}$ & 9 & 15,70 & & 3 \\
\hline HW & 7 & 19,00 & & 3 \\
\hline $\mathrm{ICH}$ & 8 & 20,70 & & 3 \\
\hline JBR & 8 & 16,90 & & 3 \\
\hline JE & 8 & 20,00 & & 3 \\
\hline JB & 5 & 25,60 & FF & 2 \\
\hline JW & 5 & 23,60 & $\mathrm{FF}$ & 2 \\
\hline JK & 9 & 22,50 & & 1 \\
\hline KS & 9 & 19,70 & $\mathrm{~F}$ & 3 \\
\hline LH & 8 & 21,60 & $F$ & 2 \\
\hline LW & 10 & 16,20 & & 3 \\
\hline LK & 9 & 18,70 & & 3 \\
\hline LXW & 6 & 20,90 & & 3 \\
\hline MF & 6 & 16,70 & & 4 \\
\hline MS & 7 & 19,00 & & 3 \\
\hline MW & 7 & 17,90 & & 4 \\
\hline MIW & 4 & 24,20 & $F$ & 2 \\
\hline NM & 6 & 24,50 & & 2 \\
\hline NT & 8 & 20,60 & & 3 \\
\hline $\mathrm{PH}$ & 6 & 18,80 & & 3 \\
\hline SK & 8 & 17,40 & $F$ & 3 \\
\hline SL & 9 & 17,50 & & 3 \\
\hline $\mathrm{SP}$ & 8 & 14,90 & & 4 \\
\hline TB & 6 & 22,40 & & 3 \\
\hline $\mathrm{TH}$ & 7 & 18,30 & & 3 \\
\hline
\end{tabular}

Tabelle 53: Ergebnisse des Kasten-Bumerang-Laufs. (Anmerkung: Die Punktzahl ist altersnormiert). (Legende: $F=$ Fehler während der Durchführung, Punktzahl 4= weit überdurchschnittlich, 3= überdurchschnittlich, 2= durchschnittlich, 1= unterdurchschnittlich, $0=$ weit unterdurchschnittlich) 
In der folgenden Tabelle 54 werden die prozentualen Anteile der Kinder mit den jeweiligen Punktzahlen angegeben.

\begin{tabular}{|c|c|c|}
\hline \multicolumn{3}{|c|}{ Kasten-Bumerang-Lauf } \\
\hline Punkte & $\begin{array}{c}\text { Anzahl der } \\
\text { Kinder }\end{array}$ & $\begin{array}{c}\% \text { der } \\
\text { Kinder }\end{array}$ \\
\hline 1 & 1 & 5 \\
\hline 2 & 6 & 30 \\
\hline 3 & 11 & 55 \\
\hline 4 & 2 & 10 \\
\hline Gesamt & $\mathbf{2 0}$ & 100 \\
\hline
\end{tabular}

Tabelle 54: Punktzahl und Anteile beim Kasten-Bumerang-Test

Auch die Kovariaten Anzahl der Sportarten, Verhältnis der Bewegungsaktivitäten, Trainingstage (in \%) und der Kasten-Bumerang-Lauf weisen für das Bewegungslernen (Gesamtskala) keinen Einfluss auf (siehe Tabelle 55).

\begin{tabular}{|l|c|c|c|}
\hline \multicolumn{1}{|c|}{ Variable } & $\mathrm{F}$ & $\mathrm{p}$ & $\mathrm{\eta}^{2}$ \\
\hline Anzahl der Sportarten & 0,40 & .55 & .05 \\
\hline Verhältnis der Bewegungsaktivitäten & 0,01 & .96 & .00 \\
\hline Trainingstage (in \%) & 2,24 & .17 & .22 \\
\hline Kasten-Bumerang-Lauf & 0,81 & .39 & .09 \\
\hline
\end{tabular}

Tabelle 55: Ergebnisse der Kovarianzanalyse für die Kovariaten

\subsubsection{Diskussion}

Die Variable Trainingserfahrung hat im Ergebnis keinen Einfluss auf das Bewegungslernen wenn der Gesamtwert der Fragebogenskala betrachtet wird und die Koordinations- und Antizipationsfähigkeit (gemessen am Kasten-Bumerang-Lauf) sowie die Anzahl der ausgeübten Sportarten, die Trainingstage in Prozent und das Verhältnis von Bewegungsaktivitäten als Kovariaten einbezogen werden.

Die Hypothese, wonach die Trainingserfahrung keinen Einfluss auf das Bewegungslernen hat, wurde bestätigt. Die unterschiedlichen Trainingsgruppen innerhalb der Versuchsgruppe sind demnach vergleichbar. Dennoch müssen die Ergebnisse mit Vorsicht interpretiert werden, da sie auf sehr kleinen Fallzahlen beruhen. Bezüglich des Bewegungstagebuchs sind die hohen Standardabweichungen als kritisch 
zu bewerten. Im Einzelfall kam es sogar vor, dass ein Kind sehr viele Sportarten (5) in der Freizeit ausübte und dass bei diesem Kind ein Verhältnis kleiner als eins gemessen wurde, das heißt, dass mehr bewegungsferne Aktivitäten gemessen wurden als bewegungsnahe. Deshalb ist bei der Interpretation der Daten zu beachten, dass die protokollierten Aktivitäten eher eine Annäherung darstellen, zumal beim Ausfüllen des Bewegungstagebuchs aus dem Gedächtnis Fehler auftreten können. Außerdem ist es möglich, dass die Eltern sozial erwünscht geantwortet haben. 


\subsection{Studie 7: Einfluss des Geschlechts auf das Bewegungslernen in der Versuchsgruppe}

Nachstehend geht es um die Variable Geschlecht und ihren Einfluss auf das Bewegungslernen. Es sollte kontrolliert werden, inwieweit andere Variablen (z.B. die Koordinationsfähigkeit) einen Einfluss zu Beginn der Untersuchung auf das spätere Ergebnis hatten. Der Hypothese nach gibt es keine Geschlechterunterschiede hinsichtlich des Bewegungslernens.

\subsubsection{Stichprobe}

Die Stichprobe weist alle Charakteristiken auf, die auch in Studie 1 bei der Versuchsgruppe gemessen wurden (siehe Kap. 8.3.1).

\subsubsection{Methode}

Der Einfluss der Variable Geschlecht auf das Bewegungslernen wurde mit einer Kovarianzanalyse bestimmt. Die Koordinationsfähigkeit, die Anzahl ausgeübter Sportarten, die Trainingstage in Prozent sowie das Verhältnis von bewegungsnahen zu bewegungsfernen Aktivitäten gingen dabei als Kovariate in die Berechnung mit ein.

Der Einfluss des Geschlechts auf das Bewegungslernen (bezüglich des Gesamtwertes der Fragebogenskala) wurde anhand der gleichen Methode wie bei der Variablen Trainingserfahrung in Kapitel 8.8.2 berechnet, mit der Ausnahme, dass die Variable Geschlecht nun der feste Faktor war.

Es wurde nur die Versuchsgruppe untersucht, da nur hier Informationen über die Qualität der Ausführung der Tennistechnik vorhanden waren.

\subsubsection{Ergebnisse der Studie 7}

In der Tab. 56 wird die deskriptive Statistik zum Faktor Geschlecht zum Bewegungslernen für die Gesamtskala angegeben. 


\begin{tabular}{|l|c|c|c|}
\hline Geschlecht & Mittelwert & $\begin{array}{c}\text { Standard- } \\
\text { abweichung }\end{array}$ & N \\
\hline weiblich & 0.30 & 0.44 & 9 \\
\hline männlich & 0.30 & 0.39 & 5 \\
\hline Gesamt & 0.30 & 0.41 & 14 \\
\hline
\end{tabular}

Tabelle 56: Mittelwerte (Standardabweichungen) des Faktors Geschlecht zum Bewegungslernen (Gesamtskala)

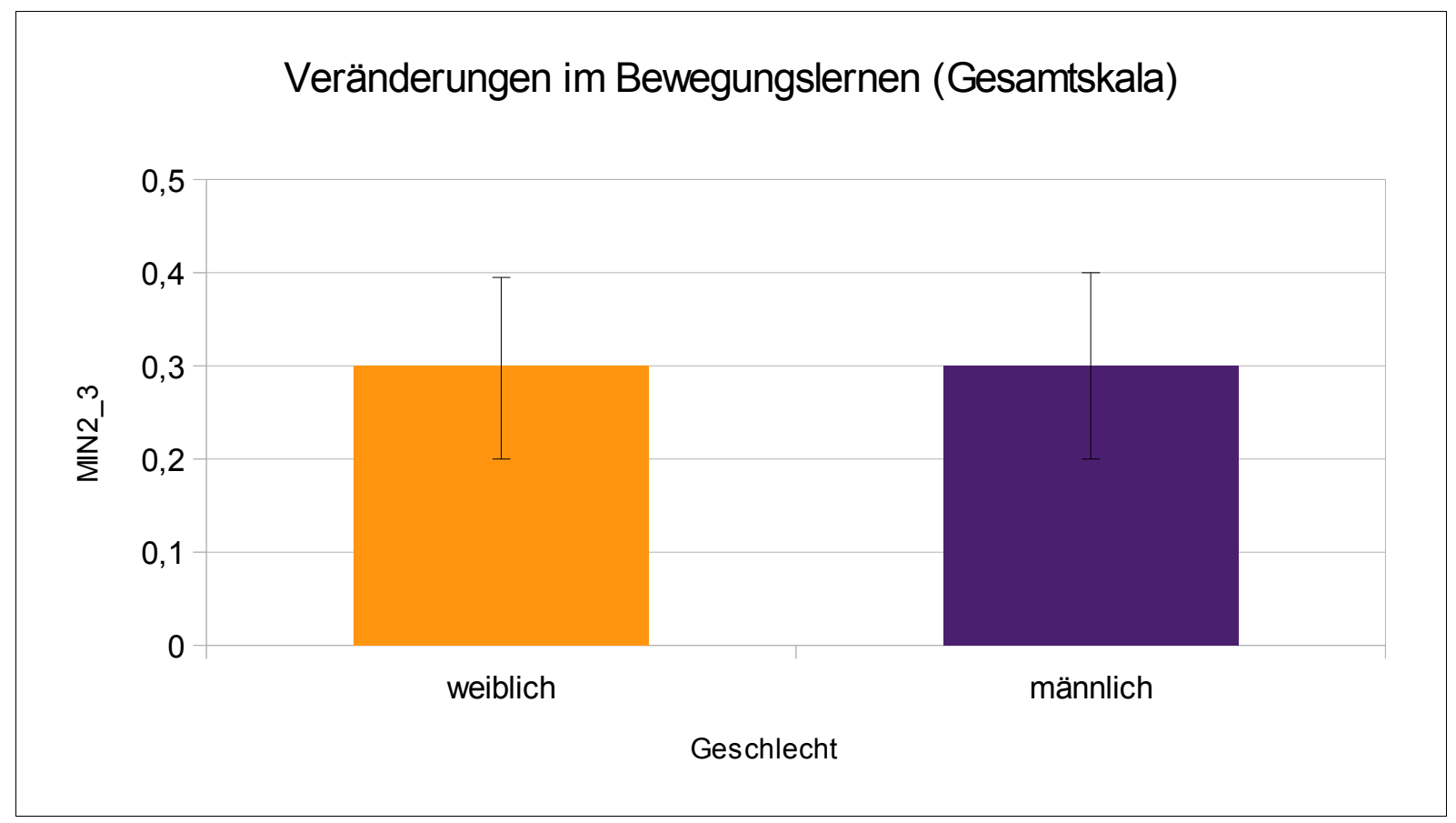

Abbildung 19: Ergebnis der Kovarianzanalyse zum Faktor Geschlecht und dessen Einfluss auf das Bewegungslernen (Gesamtskala)

Der Faktor Geschlecht war im Ergebnis der Analyse nicht signifikant $(F(1,14)=.87 ; p=.38$; $\left.\eta^{2}=.10\right)$. Das bedeutet, Jungen und Mädchen in der Versuchsgruppe wiesen keine Unterschiede im Bewegungslernen auf, wenn die Gesamtskala betrachtet wird. Die Kovariaten Anzahl der Sportarten, Verhältnis Bewegungsaktivitäten und Trainingstage (in \%) und die Koordinationsfähigkeit (gemessen am Kasten-Bumerang-Lauf) hatten ebenfalls keinen signifikanten Einfluss auf das Bewegungslernen (siehe Tabelle 57). 


\begin{tabular}{|l|c|c|c|}
\hline \multicolumn{1}{|c|}{ Variable } & $\mathrm{F}$ & $\mathrm{p}$ & $\mathrm{\eta}^{2}$ \\
\hline Anzahl der Sportarten & 1,10 & .32 & .12 \\
\hline Verhältnis der Bewegungsaktivitäten & 0,08 & .79 & .01 \\
\hline Trainingstage (in \%) & 3,18 & .11 & .28 \\
\hline Kasten-Bumerang-Lauf & 1,54 & .25 & .16 \\
\hline
\end{tabular}

Tabelle 57: Ergebnisse der Kovarianzanalyse für die Kovariaten

\subsubsection{Diskussion}

Das Geschlecht hat keinen signifikanten Einfluss auf das Bewegungs/ernen (Gesamtskala). Damit wird die eingangs aufgestellte Hypothese bestätigt. Auch die Kovariaten Anzahl der Sportarten, Verhältnis Bewegungsaktivitäten und Trainingstage (in \%) und die Koordinationsfähigkeit (gemessen am Kasten-Bumerang-Lauf) hatten keinen Einfluss auf das Bewegungslernen.

Allerdings ist eine Verzerrung der Ergebnisse möglich, da die Auswertung auf sehr kleinen Fallzahlen beruht. So könnte die Kovariate Trainingstage in Prozent bei größeren Stichprobenumfängen möglicherweise signifikant werden. 


\subsection{Allgemeine Diskussion}

Er werden im Folgenden die zentralen Fragen aus der Einleitung diskutiert. Die Reihenfolge entspricht hier der Argumentationslinie.

Es werden die Voraussetzungen für weitere Untersuchungen wie folgt erfüllt:

Die unterschiedliche Trainingserfahrung innerhalb der Versuchsgruppe wirkt sich nicht auf die Wahrnehmungs- und Vorstellungsleistung aus. Auch das unterschiedliche Training innerhalb der Versuchsgruppe wirkt sich offenbar nicht auf das Bewegungslernen (Gesamtskala) aus. Damit sind beide Teilgruppen miteinander vergleichbar und können zusammengefasst werden. Das ist hier hypothesenkonform. Außerdem sind die Eingangsvoraussetzungen in der Versuchsgruppe bezüglich der Variablen Koordinations- und Antizipationsfähigkeit (gemessen am Kasten-BumerangLauf) sowie die Anzahl der ausgeübten Sportarten, die Trainingstage in Prozent und das Verhältnis von Bewegungsaktivitäten gleich. Es ergaben sich auch keine Unterschiede bezüglich des Geschlechts und des Bewegungslernens. Dies stimmt mit der Anfangshypothese überein. Die Ergebnisse bezüglich der Wahrnehmungs- und Vorstellungsleistung können nicht mit Raten erklärt werden.

Die Eingangshypothese $H_{(F 2)}$, dass die Tenniskinder einen Vorteil bei der Lösung der Wahrnehmungs- und Vorstellungsaufgabe haben würden, wurde hier widerlegt. Als Erklärung bieten sich sowohl die Zeit für die Aktivierung des Bewegungswissens, das Wahrnehmungslernen sowie die Rolle von körperlicher Aktivität und unterschiedlicher Intentionen bei der Betrachtung an. Die Hypothese, ob sich Erfahrung bezüglich der Vorstellungsleistung auswirkt, kann jedoch nur mit differenzierter Analyse anderer Faktoren beantwortet werden: Auf diesen Zusammenhang wirken den Ergebnissen nach noch die Moderatoren Geschlecht (vgl. Studie 3) und Versuchsbedingung (vgl. Studie 4). Eine Moderatorvariable beeinflusst den Zusammenhang von zwei Merkmalen (Bortz, 1993).

Im Allgemeinen lässt sich sagen, dass der Vorstellungsbegriff eng mit dem Wahrnehmungsbegriff verbunden ist. Verschiedene Subsysteme sind bei Wahrnehmung und Vorstellung beteiligt. Daraus ergibt sich die Notwendigkeit, die jeweilige Vorstellungsaufgabe differenziert zu betrachten, weil womöglich unterschiedliche Prozesse damit verbunden sind und verschiedene neuronale Strukturen involviert sein können. Aber es gibt neben den erwähnten Gemeinsamkeiten auch Unterschiede, die etwa bei Ishai und Sagi (1997b) versucht wurden quantitativ zu erfassen (siehe Kap. 
7.2). In dieser Dissertation wurde gezeigt, dass es sowohl Unterschiede als auch Gemeinsamkeiten zwischen Wahrnehmung und Vorstellung gibt (vgl. die signifikanten und nicht signifikanten Mittelwertunterschiede in den unterschiedlichen Versuchsbedingungen). Allerdings kann die Größenordnung der Unterschiede wie in Ishai und Sagi (1997b) nicht repliziert werden. Ishai und Sagi (ebd) waren von einem Unterschied von $50 \%$ ausgegangen, das bedeutet, die Vorstellungsleistung sinkt in ihrem Versuch um die Hälfte im Vergleich zur Wahrnehmungsleistung. Die Wahrnehmungsleistung ist in dieser Dissertation in der Basisbedingung im Mittel bei 68,98 Prozent richtiger Antworten. Es verschlechtern sich die Leistungen auf 58,52 Prozent richtiger Antworten beim Test mit der Hälfte abgedeckter Bereiche („,50z“). Hierbei wird ein Unterschied von 84,83\% deutlich, das heißt die Vorstellungsleistung ist mit $84,83 \%$ so groß wie die Wahrnehmungsleistung. Allerdings steigt das Verhältnis im Versuch „50g“ auf 96,85\%.

Auch das Geschlecht scheint beim Vergleich von Wahrnehmung und Vorstellung eine Moderatorvariable zu sein. Speziell kann hervorgehoben werden, dass sich insbesondere bei den Mädchen die Versuchsbedingungen nicht voneinander unterscheiden. Dies lässt darauf schließen, dass es hier um vergleichbare Prozesse geht: Wahrnehmung und Vorstellung sind ähnlich. Bei Jungen unterscheiden sich die Wahrnehmung (Basisbedingung „0“ und die Vorstellung (Versuch „50z“). Hier treten quantifizierbare Unterschiede zwischen Wahrnehmung und Vorstellung auf (siehe Abb.16).

Problematisch muss hier angesehen werden, dass nur einige wenige Untersuchungen, die mit der Versuchsgruppe durchgeführt wurden, auch an der Kontrollgruppe erhoben werden konnten. Zudem war es nicht möglich, die Kontrollgruppe während des Sportunterrichts zu untersuchen. Außerdem kann aufgrund der unterschiedlichen Zusammensetzung der Gruppen hinsichtlich des Geschlechts der Faktor Erfahrung nur in Verbindung dieses Faktors interpretiert werden. Bezüglich einer weiteren Validierung korrelieren nur bestimmte Subskalen des Fragebogens für die Beherrschung der Tennistechnik mit den Vorstellungsfähigkeiten (siehe Kap. 8.3.3.5 und Kap. 8.3.3.6). Sie ist nur teilweise gelungen. Zusätzlich sollte die Interraterreliabilität bezüglich einiger Subskalen gesteigert werden (z.B. durch intensiviertes Training der Beobachter oder durch Verbesserung des Fragebogens). Die Berechnungen dienen nicht zuletzt aufgrund der geringen Fallzahlen der Veranschaulichung und beispielhaften Darstellung. Der korrelative Ansatz der ersten Studie (siehe Kap. 8.3) kann zudem keine 
Kausalzusammenhänge aufdecken und ist mit dem Problem von Scheinkorrelationen behaftet. Abschließend ist anzumerken, dass es sich in den weiteren Studien um ein quasi-experimentelles Design handelt. Aussagen zur Kausalität sind daher nicht möglich.

Fazit: Zusammenfassend spielt der Faktor Geschlecht zusammen mit den Faktoren Erfahrung und Versuchsbedingung bei Wahrnehmungs- und Vorstellungsleistungen in der Kindheit eine große Rolle. Es konnte gezeigt werden, dass die Methode auf der mentalen Ergänzung von abgedeckten Videosequenzen basiert. Es ist ein Verfahren, das im Rahmen von sportlichem Training bei Kindern dieses Alters zur Diagnostik von Vorstellungen von Bewegungen angewendet werden kann. Das Verfahren hat gegenüber der Mentalen Rotation den Vorteil, dass es bei Kindern dieses Alters zwischen den Geschlechtern differenzieren kann. Der Nachweis des Zusammenhangs mit Fähigkeiten, die mit dem Erlernen der Tennistechnik in Verbindung stehen, bedarf weiterer Klärung. Hierzu sollte eine weitere Validierung mittels bereits etablierter Testverfahren bzw. eine Überarbeitung des „Fragebogens zur Beherrschung der Tennistechnik" erfolgen. Für die Forschung ergibt sich mit der Untersuchung ein Nutzen hinsichtlich der Quantifizierung von Unterschieden von Wahrnehmung und Vorstellung von Bewegungen. Diese wurde im Hinblick auf das Geschlecht differenziert. 


\subsection{Ausblick für weitere Forschung}

In einer weiterführenden Studie würden folgende Ergänzungen von Vorteil sein:

1. Die Kinder der Versuchsgruppe und die der Kontrollgruppe sollten vor dem Training untersucht werden, da sie dann nicht erschöpft sind.

2. Zudem ist eine Studie mit größeren Stichproben anzuraten, um speziellere Auswertungen anzustellen, die größere Fallzahlen in den einzelnen Zellen bedürfen.

3. Eine Balancierung des Geschlechts in den Gruppen ist durchzuführen.

4. Eine Weiterentwicklung des Quasi-Experiments wäre möglich, wenn Stichproben zufällig auf die Versuchsbedingungen zugeteilt werden könnten, d.h. randomisiert werden.

Wie aus dieser und anderen Forschungsarbeiten erkennbar ist, ergeben sich neue Fragestellungen, die im Folgenden kurz skizziert werden:

5. Weitere Alternativerklärungen sollten experimentell untersucht werden. Der Vergleich mit noch älteren Kindern bzw. Jugendlichen und Erwachsenen gäbe beispielsweise Aufschluss darüber, inwieweit tatsächlich eine Merkstrategie bei dieser Art von Aufgabenstellung bevorzugt wird.

6. Gibt es einen Gedächtniseffekt? Die Dauer der Präsentation der Videos oder auch das Zeitintervall zwischen den Videos als Faktor mit einzubeziehen heißt, die Gedächtniskomponente zu betrachten: Erfahrene Sportler sollten bei längerem Zeitintervall besser abschneiden als junge Sportler.

7. Gibt es einen Vertrautheitseffekt, d.h. können Kinder die eigenen Videos besser erkennen als wenn eine fremde Person auf den Videos gezeigt wird?

Das Ziel weiterer Forschung sollte u.a. auch das Finden einer optimalen Bildabdeckung sein. 


\section{Anhang}

\subsection{Bewegungstagebuch und Fragebogen zur Erfassung der Tennistechnik}

Im Folgenden wird das Bewegungstagebuch dargestellt. Es wurde für die Versuchsgruppe eingesetzt. Die vierten Seite des Tagebuchs stimmt mit der dritten Seite überein.

Anschließend wird der „Fragebogen zur Erfassung der Tennistechnik“ dargestellt. 


\section{Musterplan/Beispiel}

Vorname, Name des Kindes: Max Mustermann

Datum:

Regelmäßige Termine: Bitte auch Hobbys eintragen, die früher, aber über einen längeren Zeitraum betrieben wurden.

Vereinssport/Training

\begin{tabular}{|r|l|l|l|}
\hline \multicolumn{1}{|c|}{ Sportart } & \multicolumn{1}{|c|}{$\begin{array}{c}\text { Wöchentliche Termine } \\
\text { (Wann, wie lange?) }\end{array}$} & $\begin{array}{c}\text { Seit wann/wie lange } \\
\text { betreibt/betrieb das Kind } \\
\text { diese Sportart? }\end{array}$ \\
\hline 1 & Jüngsten-Tennis & samstag, 9:00-10:30 & seit Mai 2004 \\
\hline 2 & Fußball & freitags, 14:30-16:00 & seit Sommer 2000 \\
\hline 3 & Basketball & 90 Minuten wöchentlich & $\begin{array}{l}\text { von 2001-2002, } \\
\text { eineinhalb Jahre }\end{array}$ \\
\hline 5 & & & \\
\hline
\end{tabular}

Musik und Gestalterisches (z.B. Flöten, Gitarre, Malkurs, Töpferkurs, .... )

\begin{tabular}{|c|c|c|c|}
\hline & Sportart & $\begin{array}{l}\text { Wöchentliche Termine } \\
\text { (Wann, wie lange?) }\end{array}$ & $\begin{array}{c}\text { Seit wann/wie lange } \\
\text { betreibt/betrieb das Kind } \\
\text { diese Beschäftigung? }\end{array}$ \\
\hline 1 & Flötenunterricht & montags, von 14-14:45 & seit 2 Jahren \\
\hline 2 & Kunst-AG & mittwochs von 13:30-14:15 & seit Sommer 2004 \\
\hline 3 & Klavierunterricht & $\begin{array}{l}\text { montags, 16-16:45 und } \\
\text { donnerstags, 16-16:45 }\end{array}$ & seit Juni 2001 \\
\hline 4 & & & \\
\hline
\end{tabular}




\section{Allgemeine Erfassung des Tagesablaufs des Kindes}

Vorname, Name des Kindes:

Datum:

Regelmäßige Termine: Bitte auch Hobbys eintragen, die früher, aber über einen längeren Zeitraum betrieben wurden.

\section{Vereinssport/Training}

\begin{tabular}{|r|r|c|c|}
\hline \multicolumn{1}{|c|}{ Sportart } & $\begin{array}{c}\text { Wöchentliche Termine } \\
\text { (Wann, wie lange?) }\end{array}$ & $\begin{array}{c}\text { Seit wann/wie lange } \\
\text { betreibt/betrieb das Kind } \\
\text { diese Sportart? }\end{array}$ \\
\hline 1 & & & \\
\hline 3 & & & \\
\hline 4 & & & \\
\hline 5 & & & \\
\hline
\end{tabular}

Musik und Gestalterisches (z.B. Flöten, Gitarre, Malkurs, Töpferkurs, .... )

\begin{tabular}{|r|l|c|c|}
\hline & Sportart & $\begin{array}{c}\text { Wöchentliche Termine } \\
\text { (Wann, wie lange?) }\end{array}$ & $\begin{array}{c}\text { Seit wann/wie lange } \\
\text { betreibt/betrieb das Kind } \\
\text { diese Beschäftigung? }\end{array}$ \\
\hline 1 & & & \\
\hline 3 & & & \\
\hline 4 & & & \\
\hline
\end{tabular}




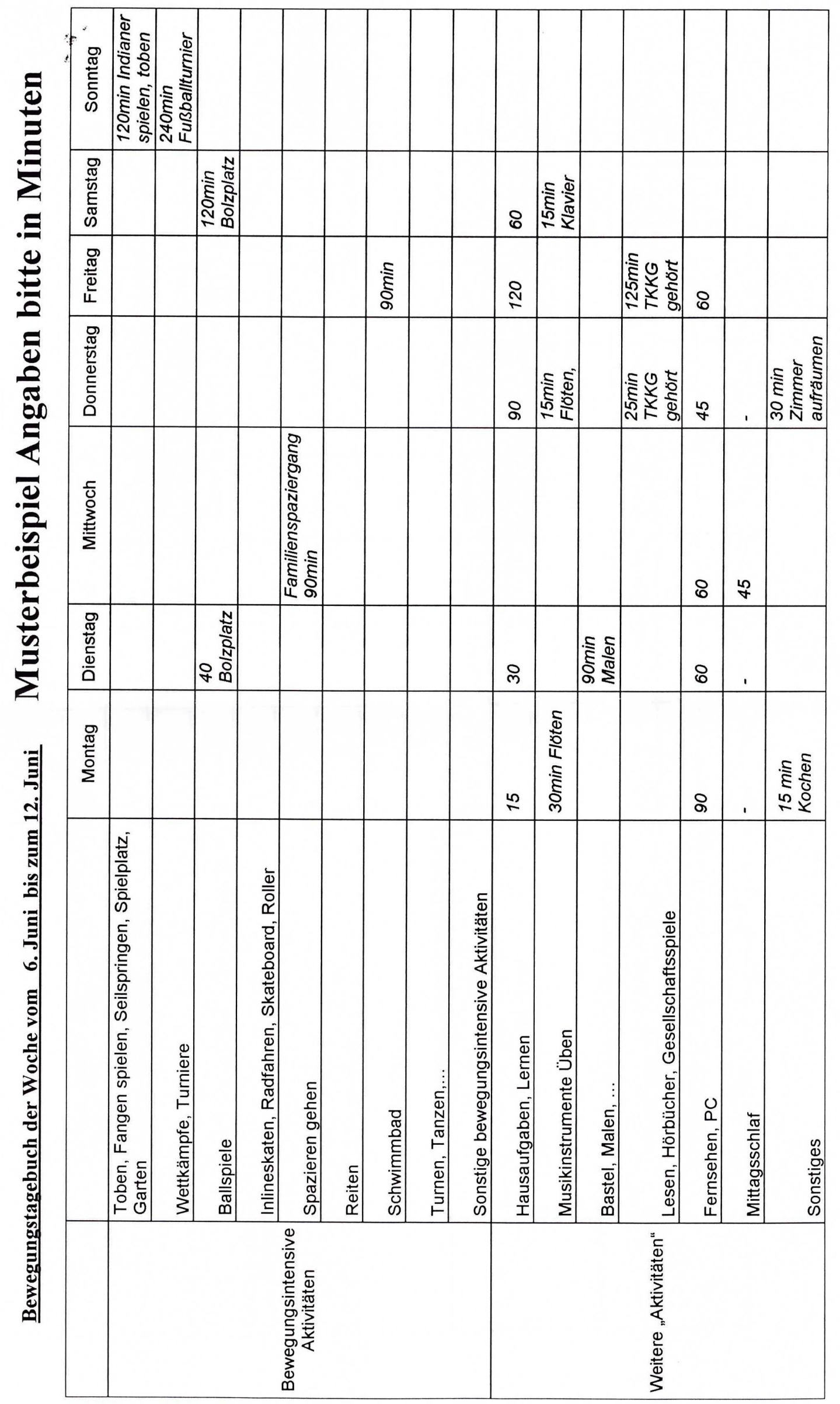


Fragebogen zur Erfassung der Tennistechnik Nummer des Videos:E.../MZP..../K....

Vielen Dank dafür, dass Sie sich etwas Zeit nehmen, um die folgenden Fragen zu beantworten. Sie werden jetzt Videoaufnahmen von Kindern sehen, bei denen wir sie jeweils um Ihr Urteil zu den untenstehenden Fragen bitten. Bitte denken Sie bei Ihrem Urteil nicht allzu lange nach; die Fragen zielen auf Ihren persönlichen Eindruck ab. Daher gibt es auch keine richtigen oder falschen Antworten. Der Fragebogen wird anonym ausgewertet, es werden keine Rückschlüsse auf Ihre Person gezogen.

Sie sehen nun eine Dreierserie des Vorhand-Grundschlags. Bitte beurteilen Sie auf einer Skala von 1 bis 4 ( $1=$ trifft $\mathrm{zu} /$ sehr gut, $2=$ trifft eher zu/gut, $3=$ trifft eher nicht $\mathrm{zu}, 4=$ trifft nicht $\mathrm{zu}$ ), wie gut die folgenden Merkmale 1 bis 5 ausgeführt sind. Sie können die drei Schläge so oft Sie wollen anschauen. Kreuzen Sie bitte an:

\section{Rechtzeitiges Einnehmen der Schlagposition $\quad 1-2-3-4$}

\section{Ausholbewegung:}

rechtzeitiger Beginn der Ausholbewegung $\quad 1-2-3-4$

rechtzeitiger Beginn der Schlagphase $\quad 1-2-3-4$

\section{Schlagbewegung:}

$\begin{array}{lc}\text { Griffhaltung } & 1-2-3-4 \\ \text { Treffpunkt } & 1-2-3-4 \\ \text { Fußstreckung } & 1-2-3-4 \\ \text { Kniestreckung } & 1-2-3-4 \\ \text { Ganzkörperstreckung } & 1-2-3-4 \\ \text { Vorwärts-Aufwärts des Schlägerkopfes } & 1-2-3-4 \\ \text { Wischbewegung } & 1-2-3-4\end{array}$

4. Ausschwungbewegung flüssiges Durchschwingen/kein Auffangen $1-2-3-4$

Bitte sehen Sie sich die drei Schläge eines Kindes an und beurteilen Sie auf der Skala von 1 bis 4 (1=sehr konstant, 2=konstant, 3= variabel, 4= stark variabel) wie konstant das Kind spielt.

\section{Bewegungskonstanz $1-2-3-4$}

Bitte beschreiben Sie Auffälligkeiten bei diesem Kind: 


\subsection{Einzelfallbetrachtung}

Folgend werden die Werte für das Bewegungslernen, die aus dem „Fragebogen zur Erfassung der Tennistechnik" ermittelt wurden, für drei Messzeitpunkte pro Kind dargestellt.

\section{Bewegungslernen}

Kind $A B$

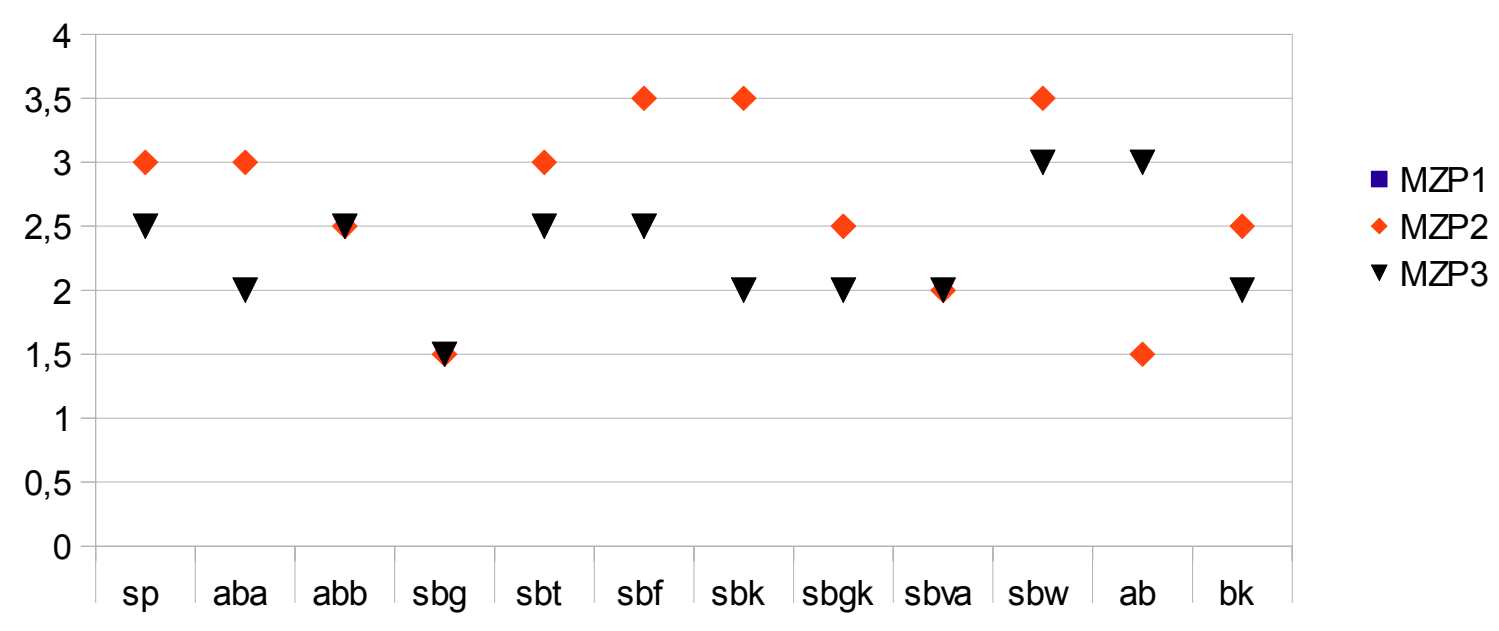

Abbildung 20: Entwicklung der Tennistechnik über 3 Messzeitpunkte bei Kind AB

\section{Bewegungslernen}

Kind BL

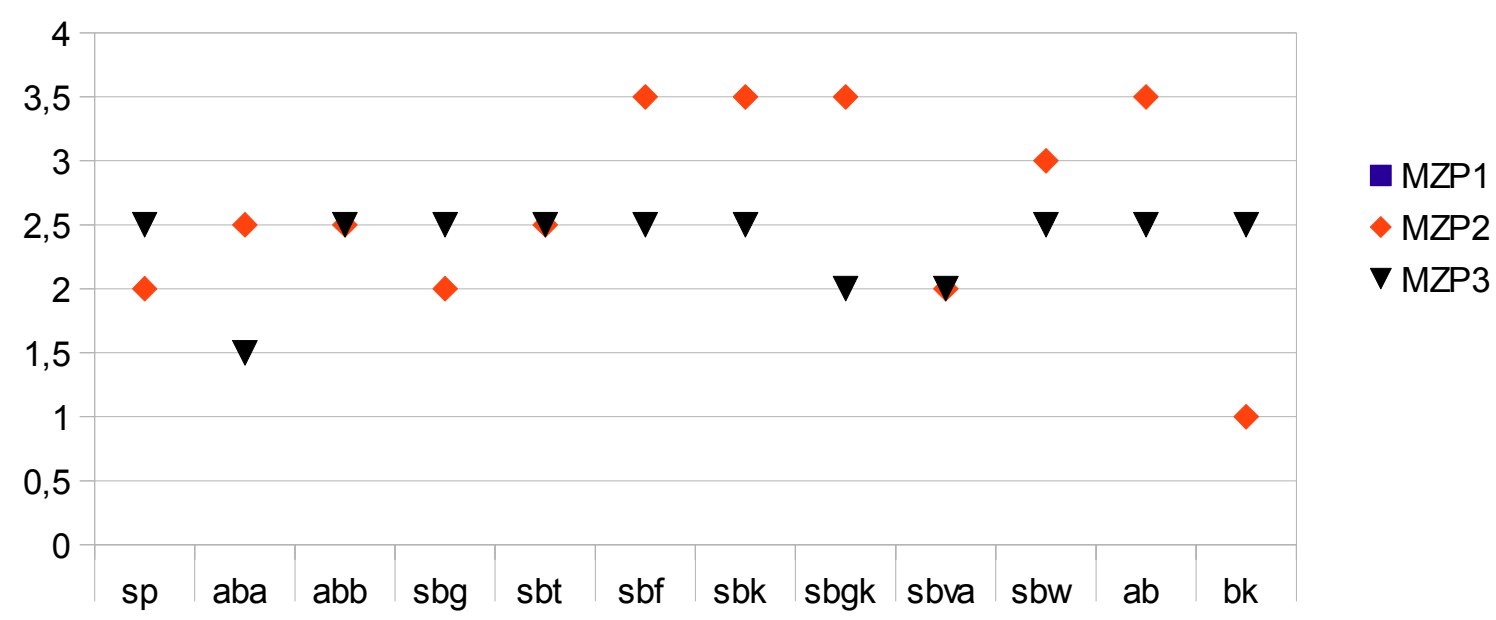

Abbildung 21: Entwicklung der Tennistechnik über 3 Messzeitpunkte bei Kind BL 


\section{Bewegungslernen}

Kind BT

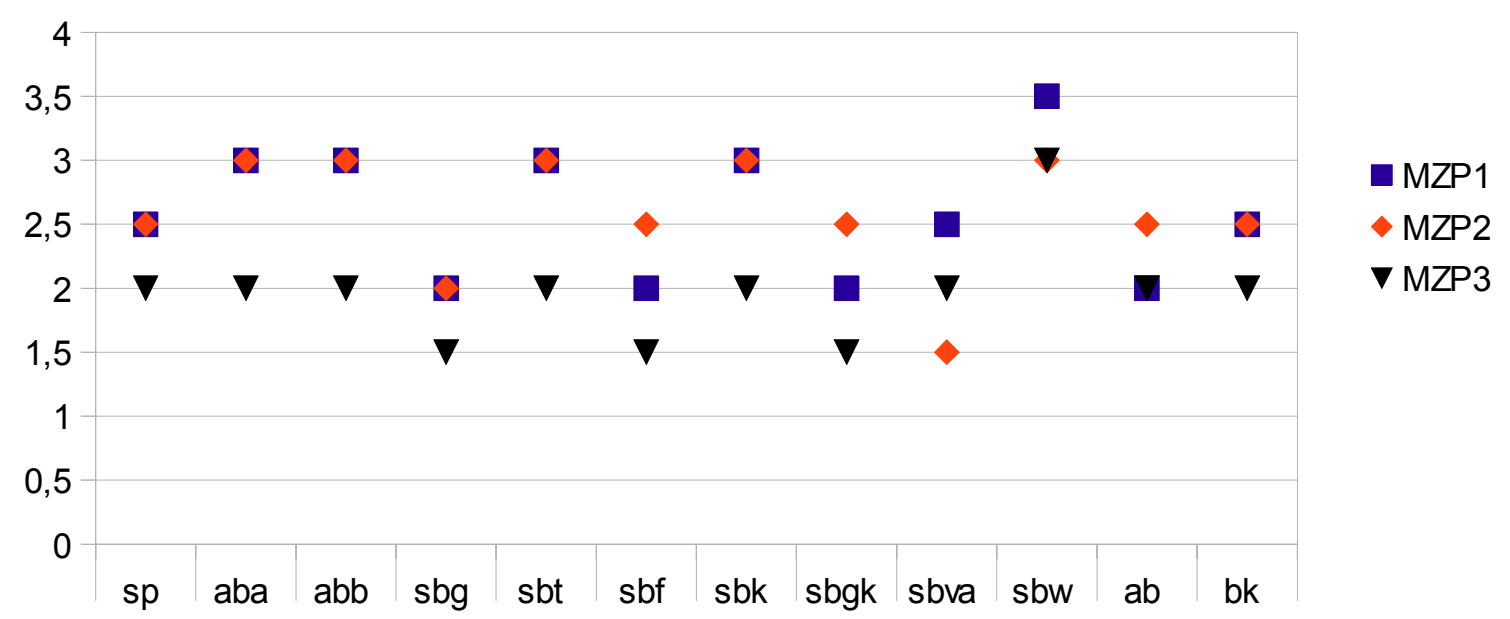

Abbildung 22: Entwicklung der Tennistechnik über 3 Messzeitpunkte bei Kind BT

\section{Bewegungslernen}

Kind DH

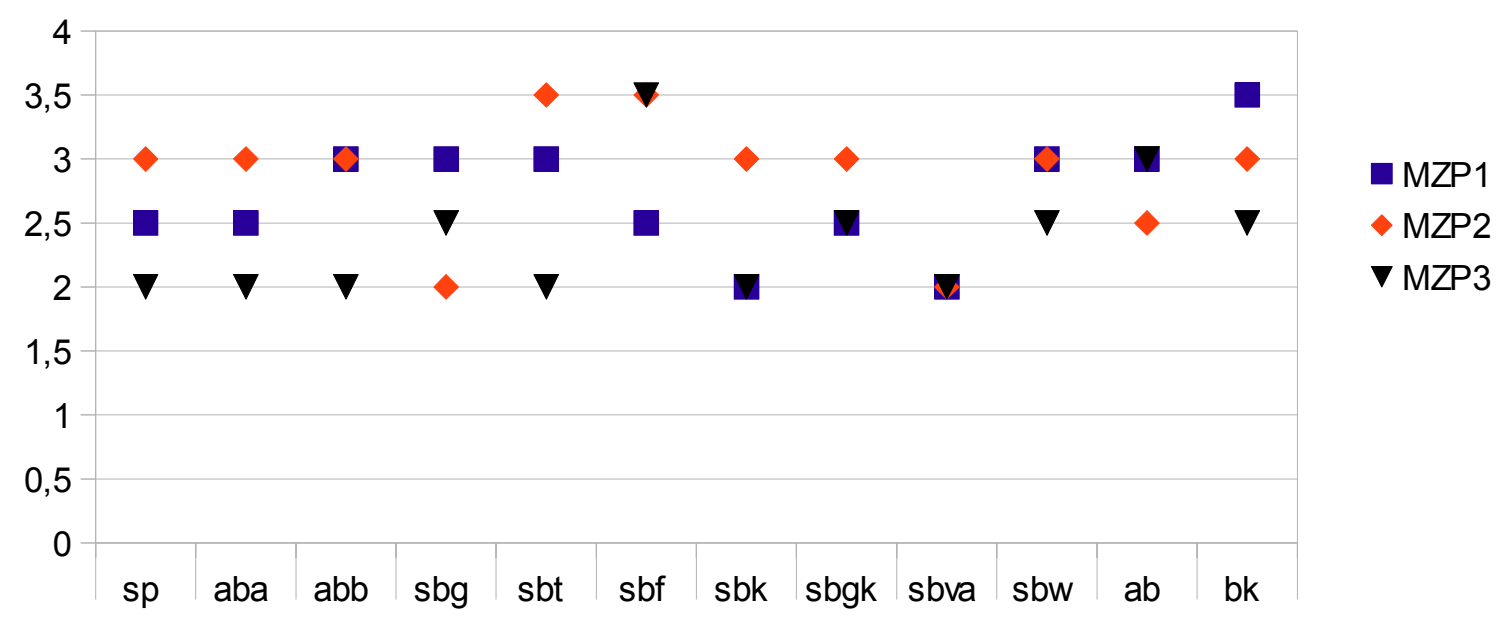

Abbildung 23: Entwicklung der Tennistechnik über 3 Messzeitpunkte bei Kind DH 


\section{Bewegungslernen}

Kind FB

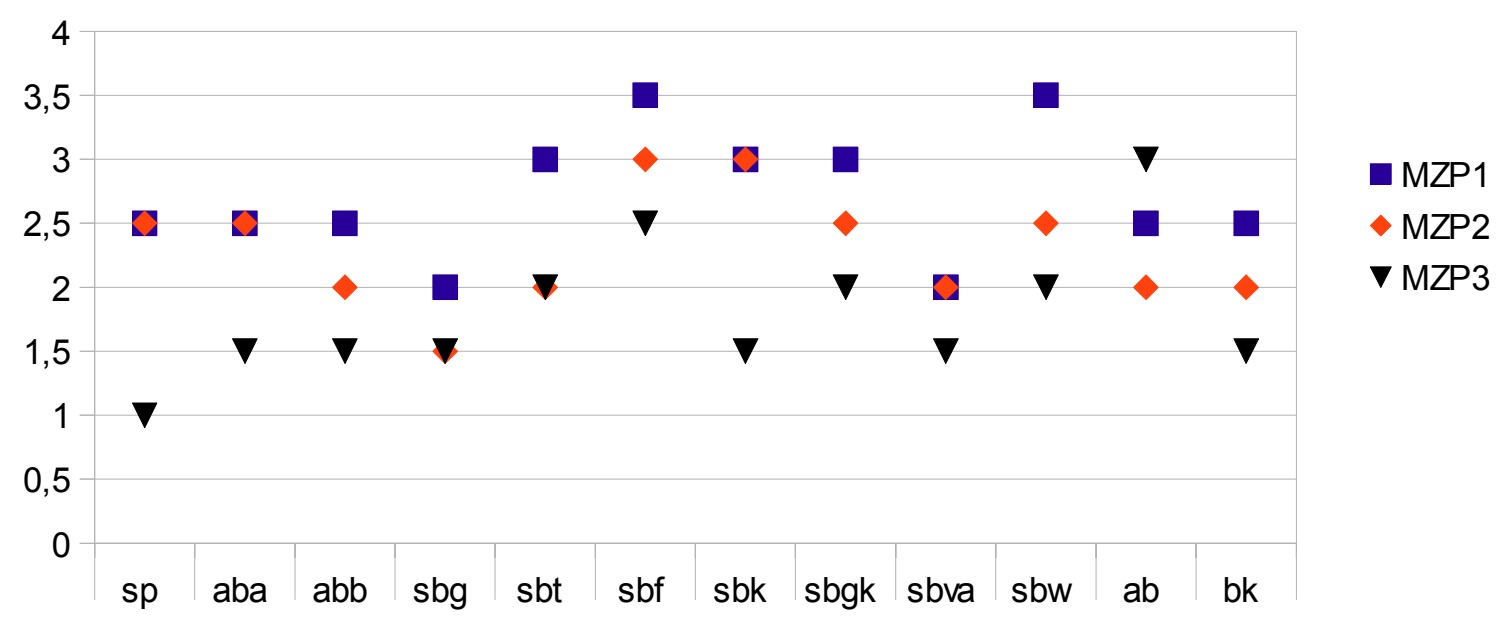

Abbildung 24: Entwicklung der Tennistechnik über 3 Messzeitpunkte bei Kind FB

\section{Bewegungslernen}

Kind FL

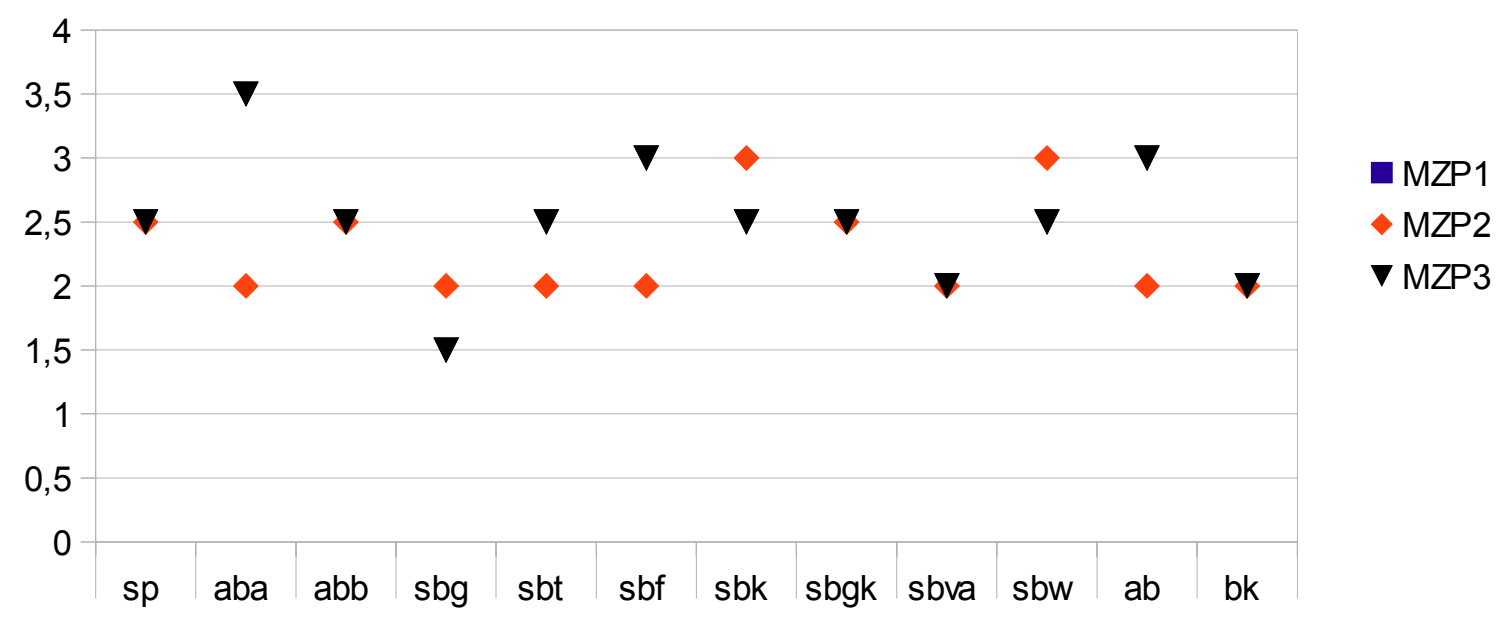

Abbildung 25: Entwicklung der Tennistechnik über 3 Messzeitpunkte bei Kind FL 


\section{Bewegungslernen}

Kind GK

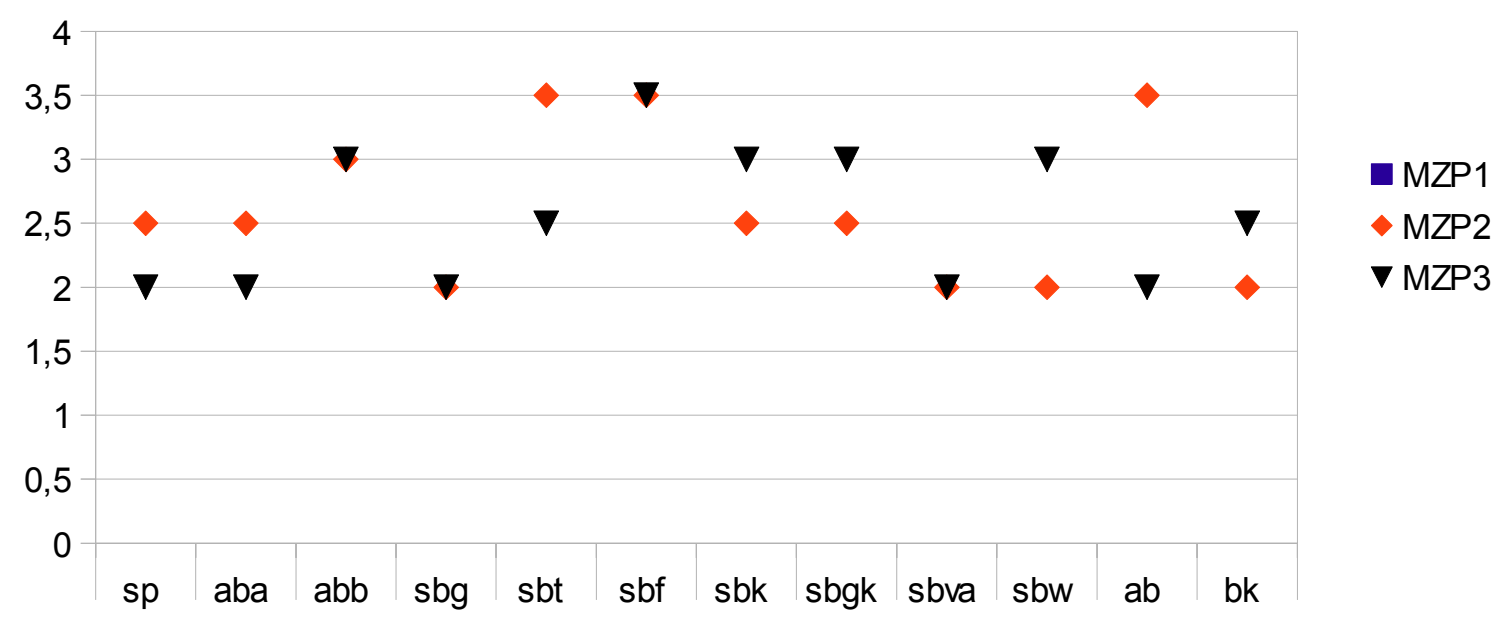

Abbildung 26: Entwicklung der Tennistechnik über 3 Messzeitpunkte bei Kind GK

\section{Bewegungslernen}

Kind $\mathrm{HB}$

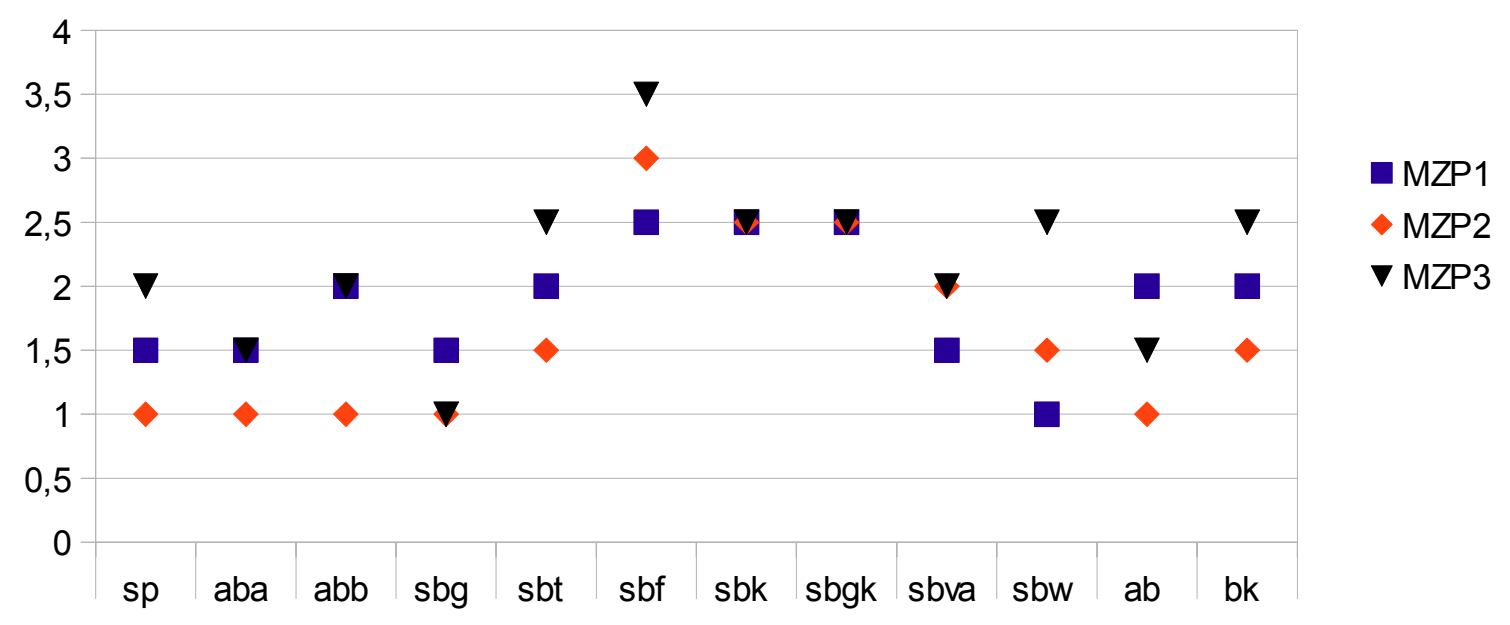

Abbildung 27: Entwicklung der Tennistechnik über 3 Messzeitpunkte bei Kind HB 


\section{Bewegungslernen}

Kind HW

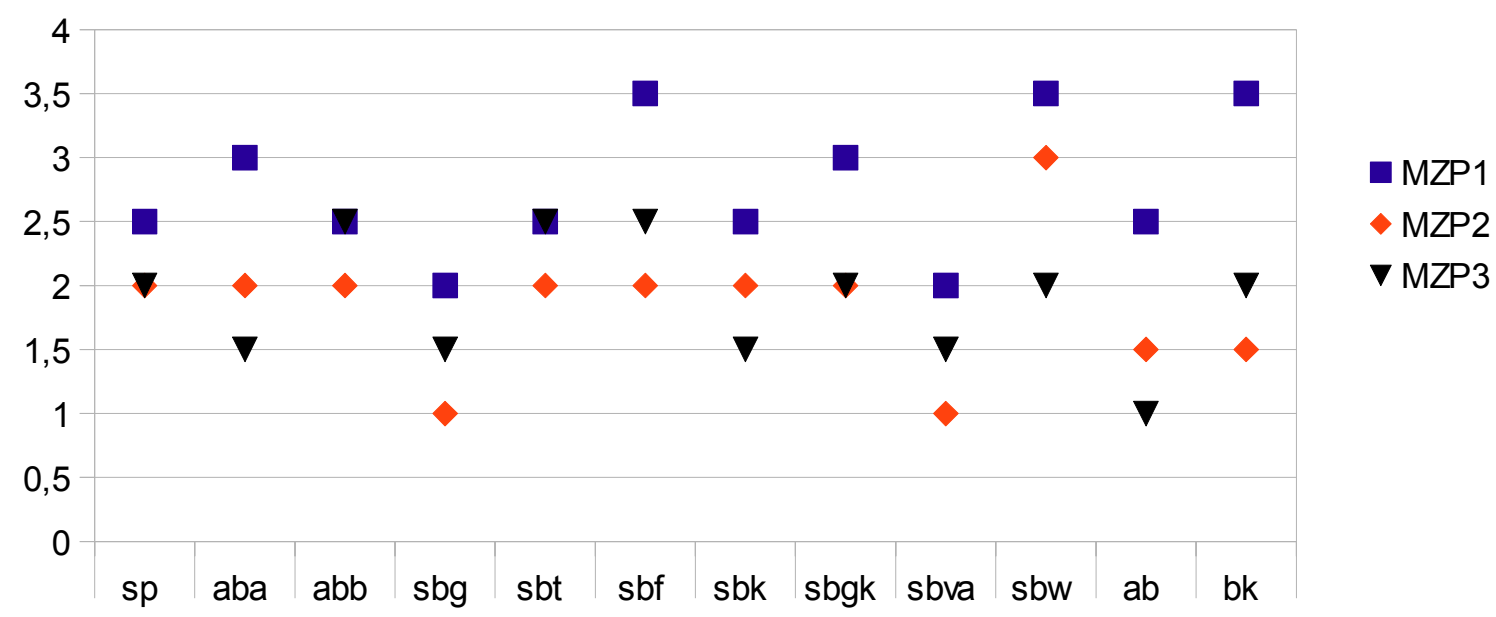

Abbildung 28: Entwicklung der Tennistechnik über 3 Messzeitpunkte bei Kind HW

\section{Bewegungslernen}

Kind $\mathbb{H}$

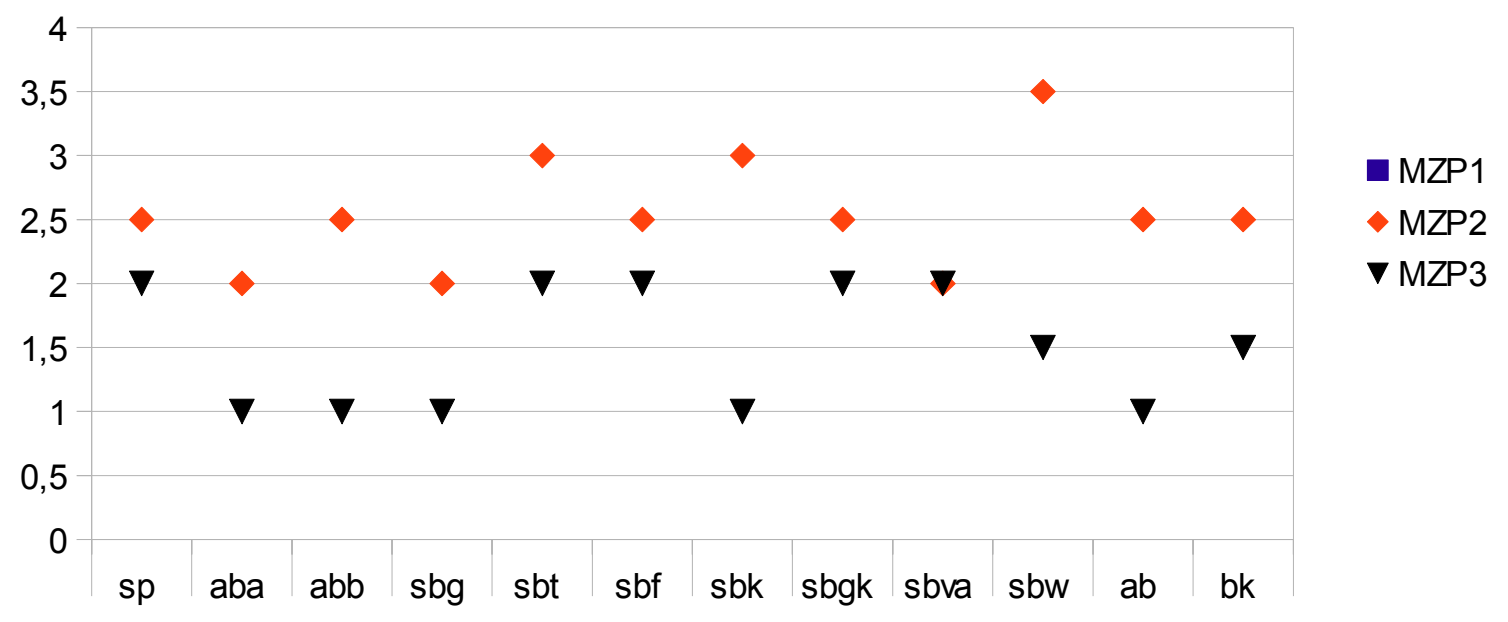

Abbildung 29: Entwicklung der Tennistechnik über 3 Messzeitpunkte bei Kind IH 


\section{Bewegungslernen}

\section{Kind KB}

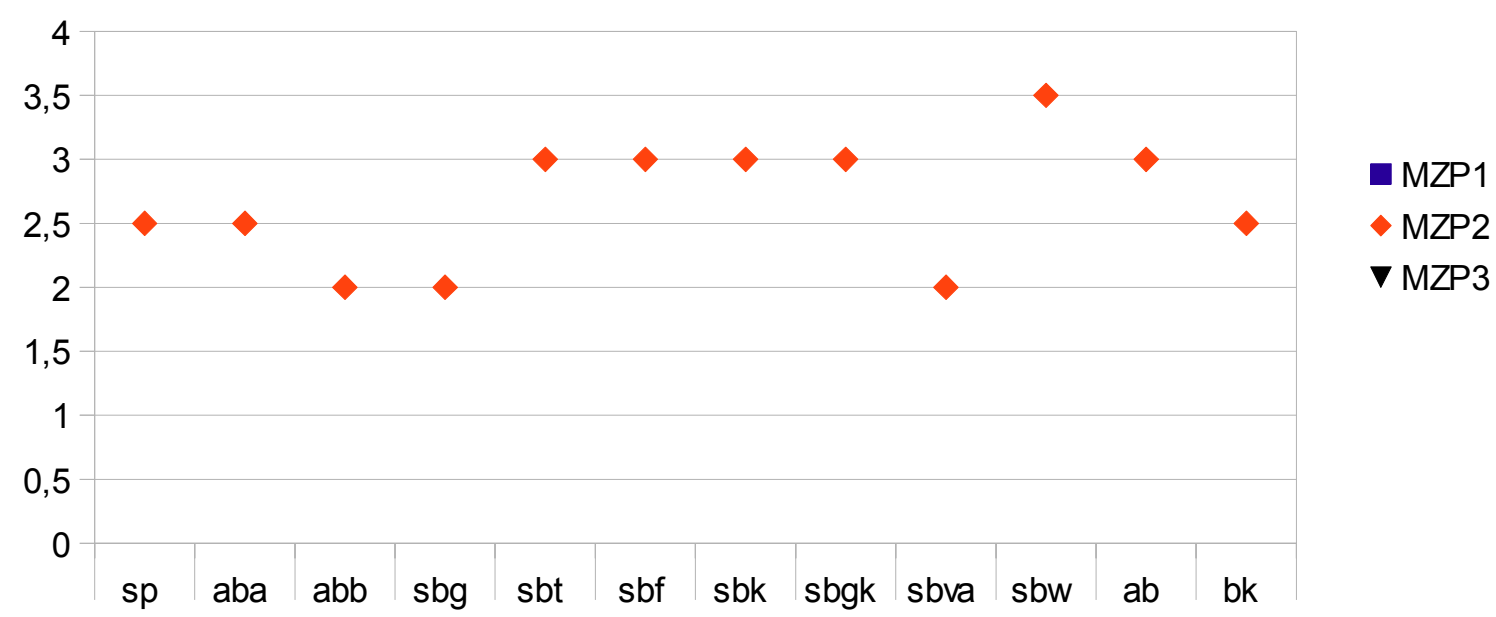

Abbildung 30: Entwicklung der Tennistechnik über 3 Messzeitpunkte bei Kind KB

\section{Bewegungslernen}

\section{Kind KS}

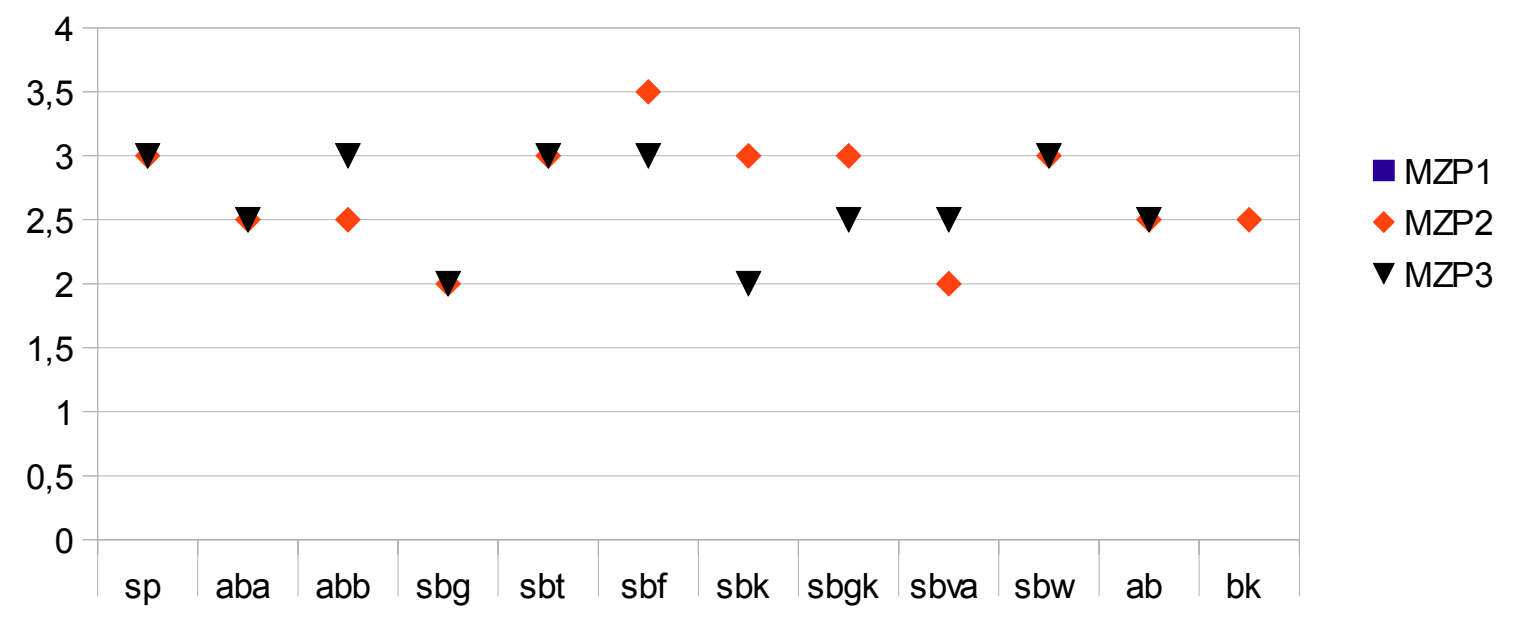

Abbildung 35: Entwicklung der Tennistechnik über 3 Messzeitpunkte bei Kind KS 


\section{Bewegungslernen}

\section{Kind LH}

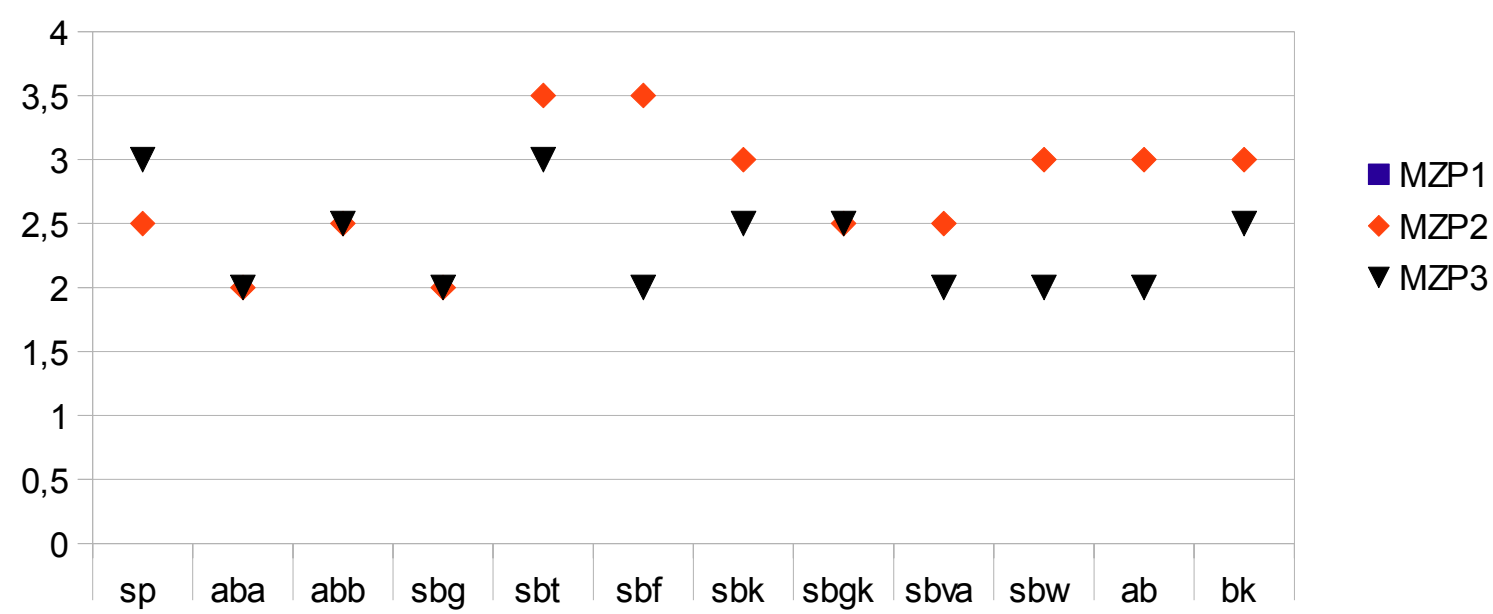

Abbildung 36: Entwicklung der Tennistechnik über 3 Messzeitpunkte bei Kind LH

\section{Bewegungslernen}

Kind LR

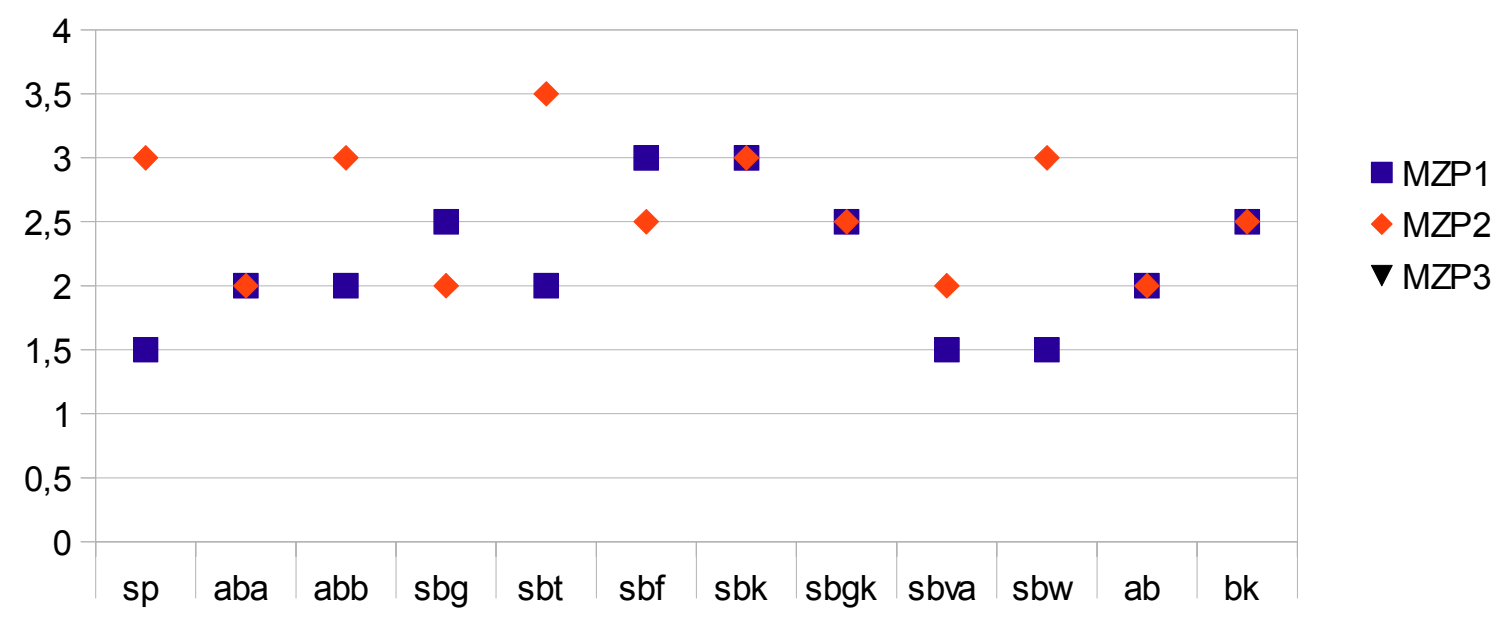

Abbildung 37: Entwicklung der Tennistechnik über 3 Messzeitpunkte bei Kind LR 


\section{Bewegungslernen}

\section{Kind LW}

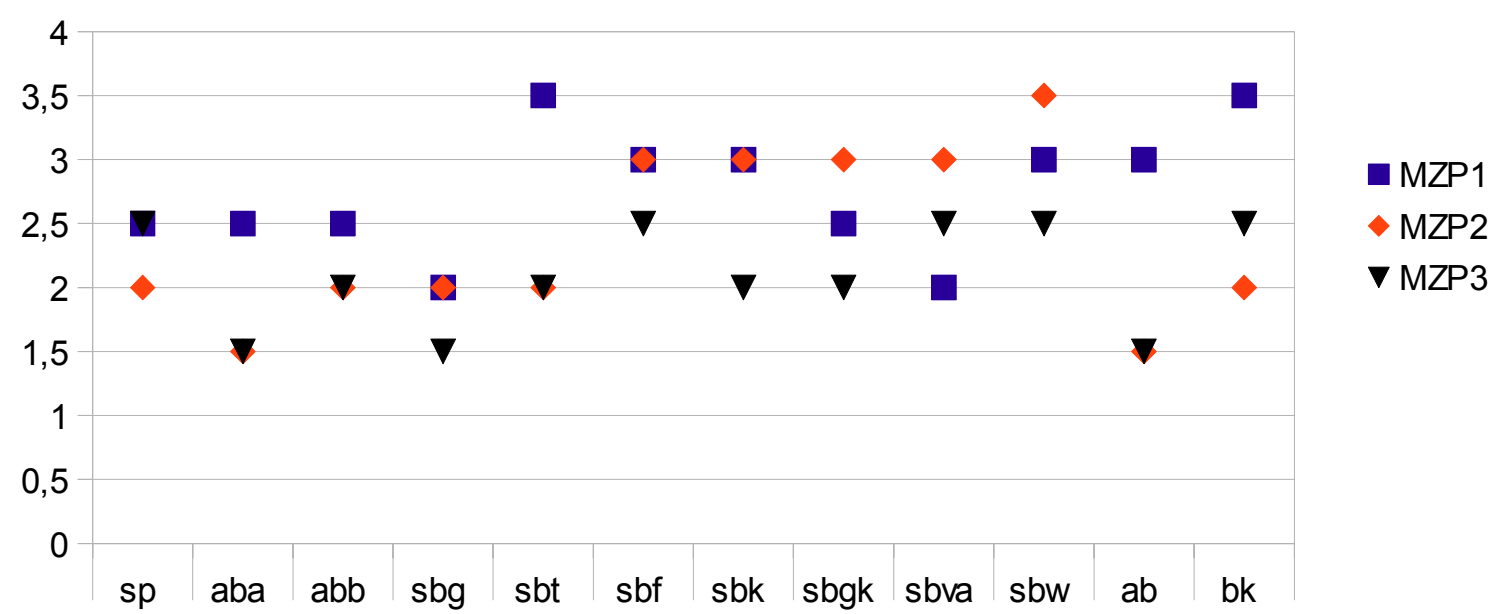

Abbildung 38: Entwicklung der Tennistechnik über 3 Messzeitpunkte bei Kind LW

\section{Bewegungslernen}

Kind LK

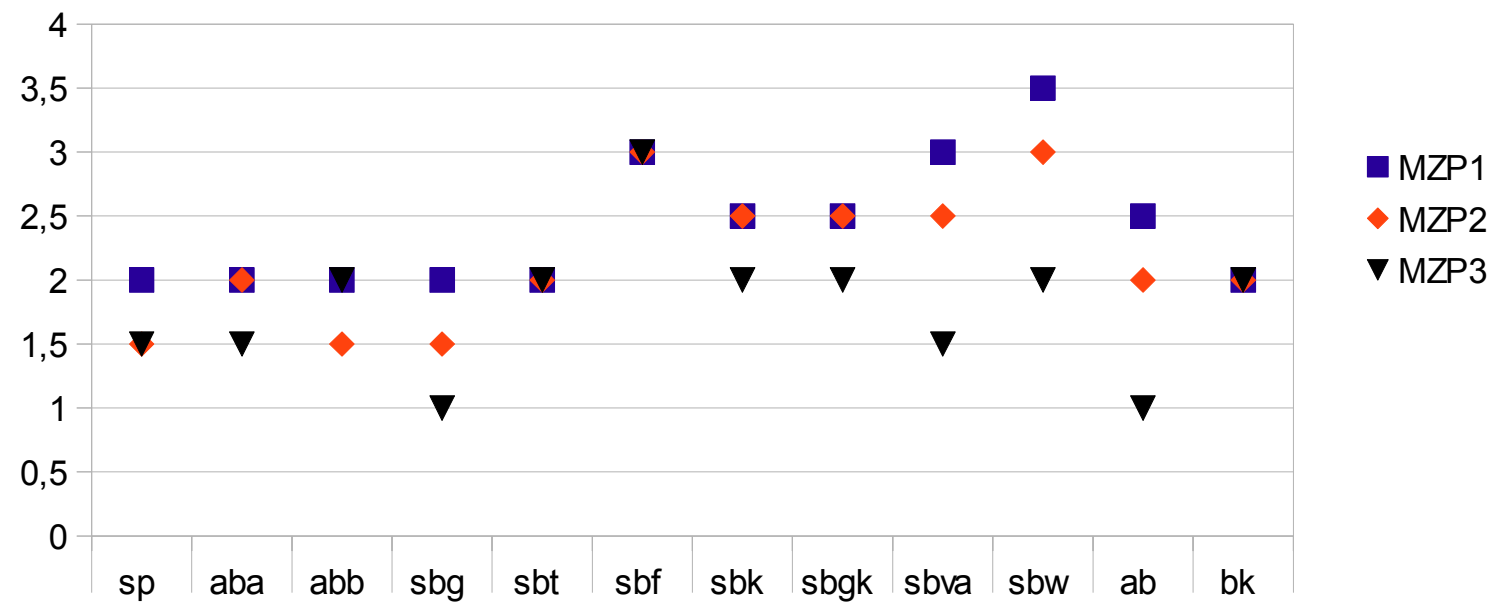

Abbildung 39: Entwicklung der Tennistechnik über 3 Messzeitpunkte bei Kind LK 


\section{Bewegungslernen}

\section{Kind MAS}

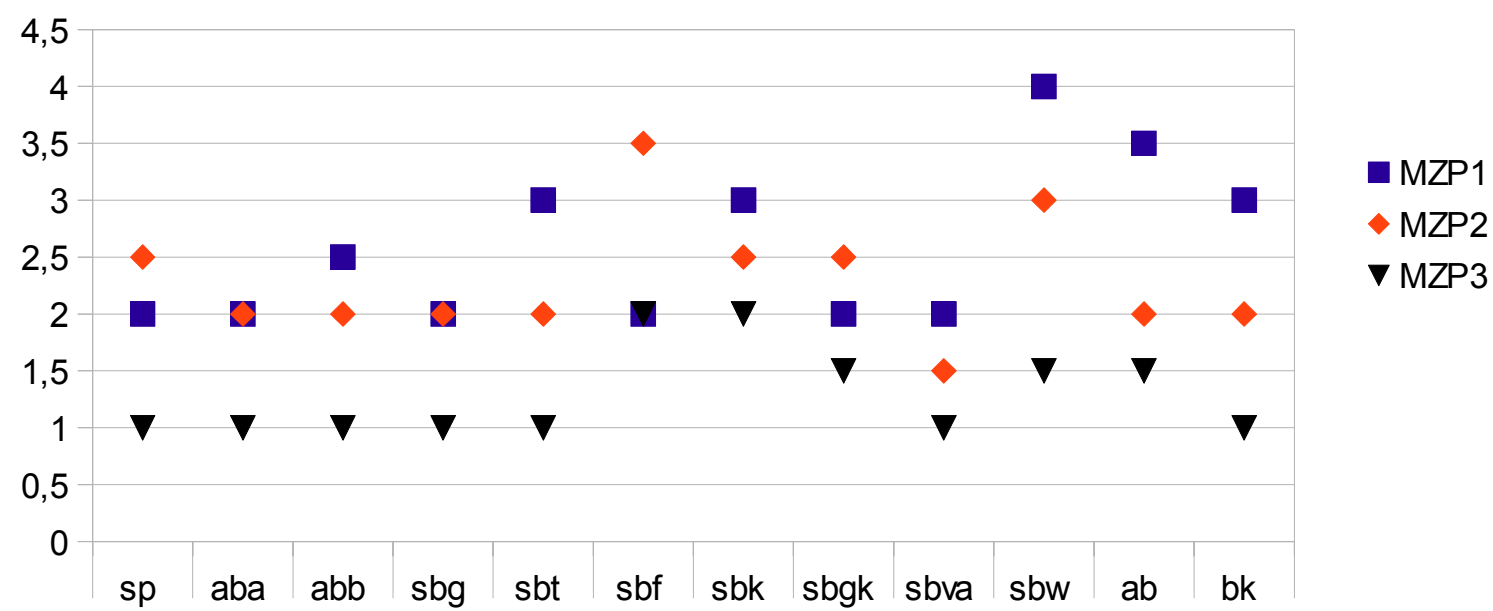

Abbildung 40: Entwicklung der Tennistechnik über 3 Messzeitpunkte bei Kind MAS

\section{Bewegungslernen}

Kind MS

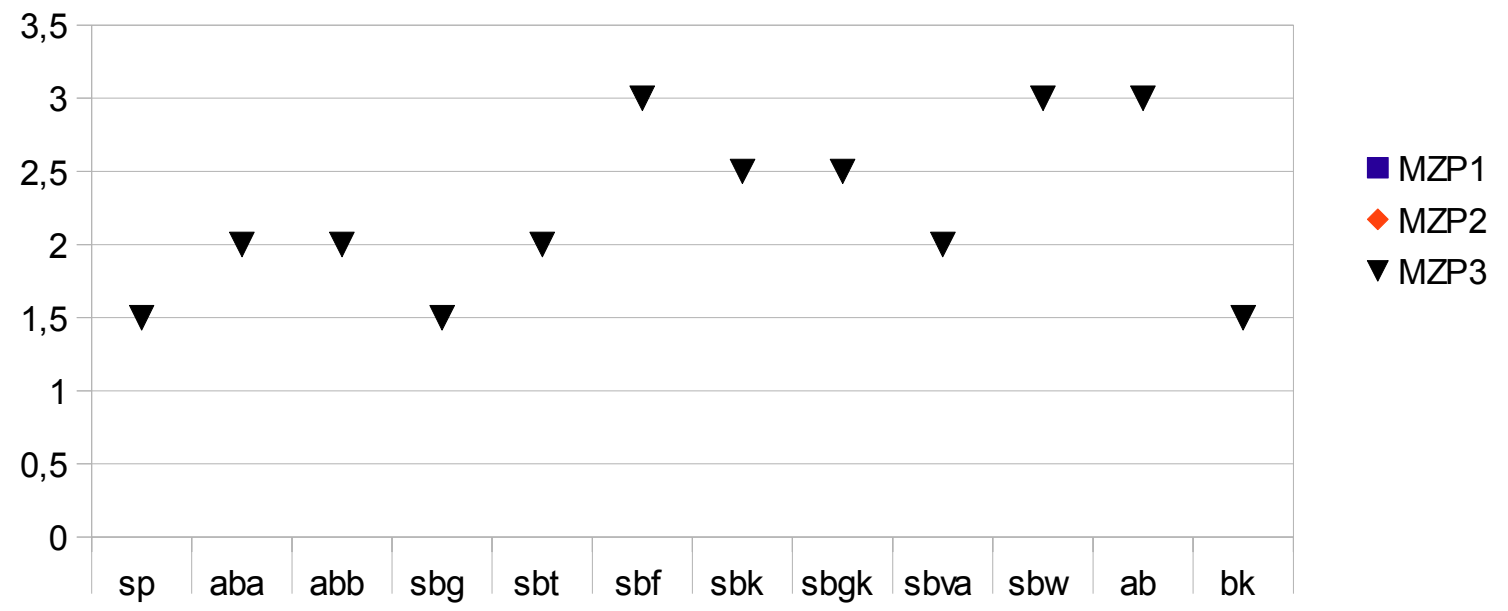

Abbildung 41: Entwicklung der Tennistechnik über 3 Messzeitpunkte bei Kind MS 


\section{Bewegungslernen}

Kind MW

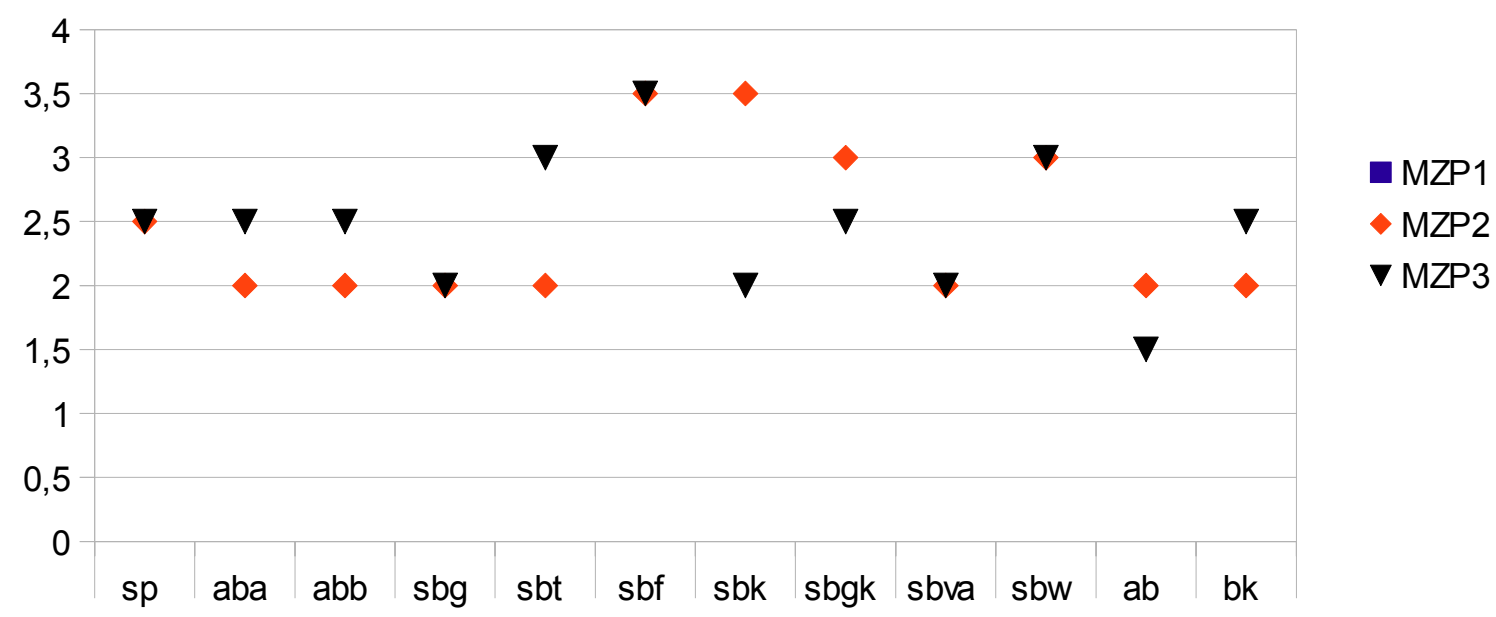

Abbildung 43: Entwicklung der Tennistechnik über 3 Messzeitpunkte bei Kind MW

\section{Bewegungslernen}

\section{Kind NM}

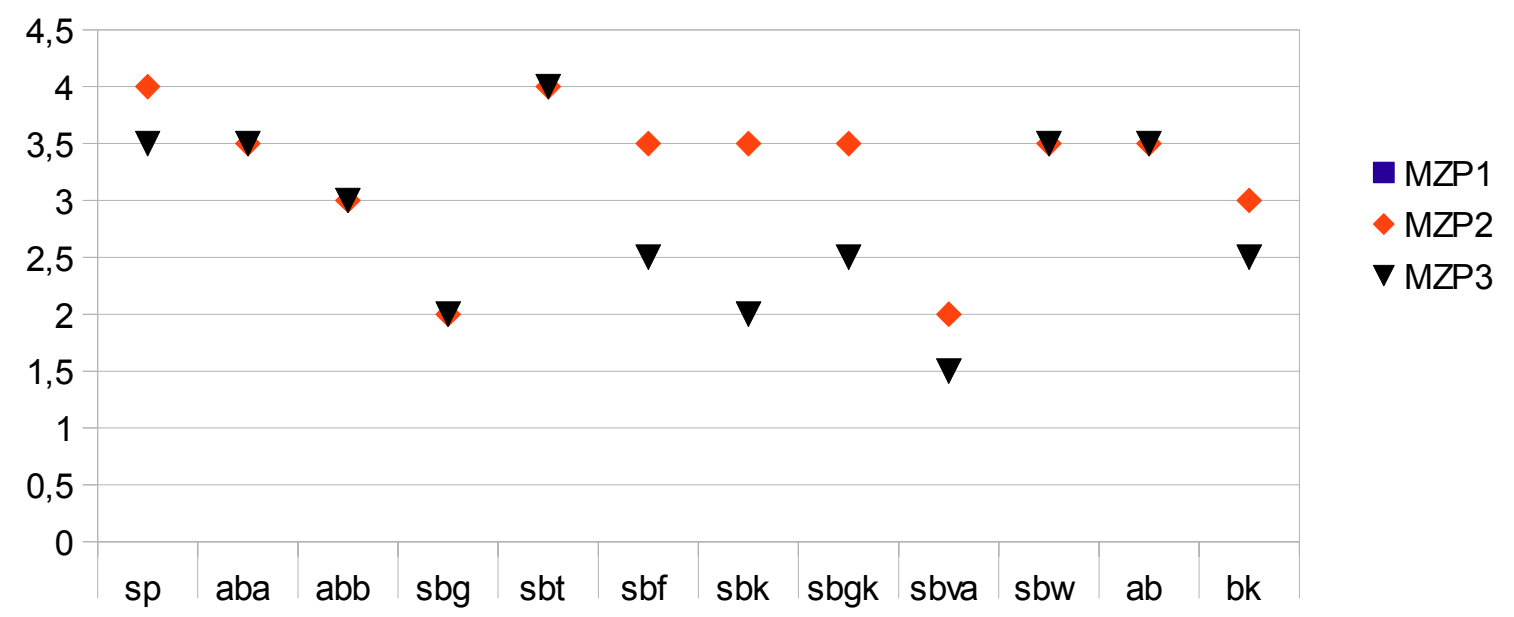

Abbildung 44: Entwicklung der Tennistechnik über 3 Messzeitpunkte bei Kind NM 


\section{Bewegungslernen}

\section{Kind NT}

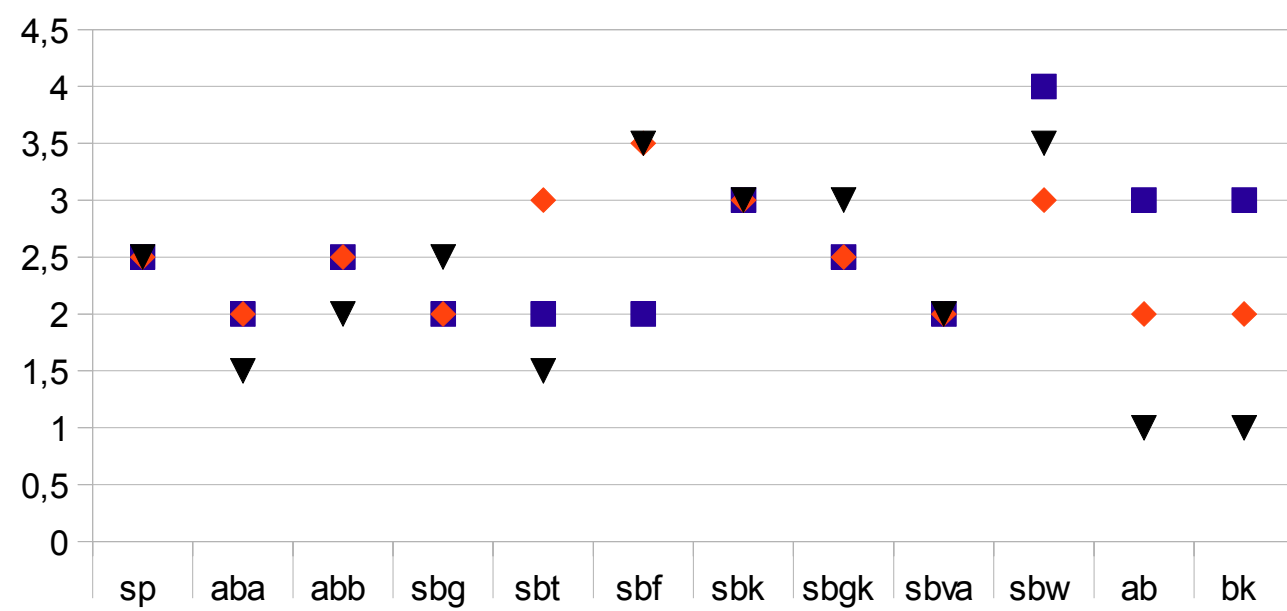

Abbildung 45: Entwicklung der Tennistechnik über 3 Messzeitpunkte bei Kind NT

\section{Bewegungslernen}

Kind NV

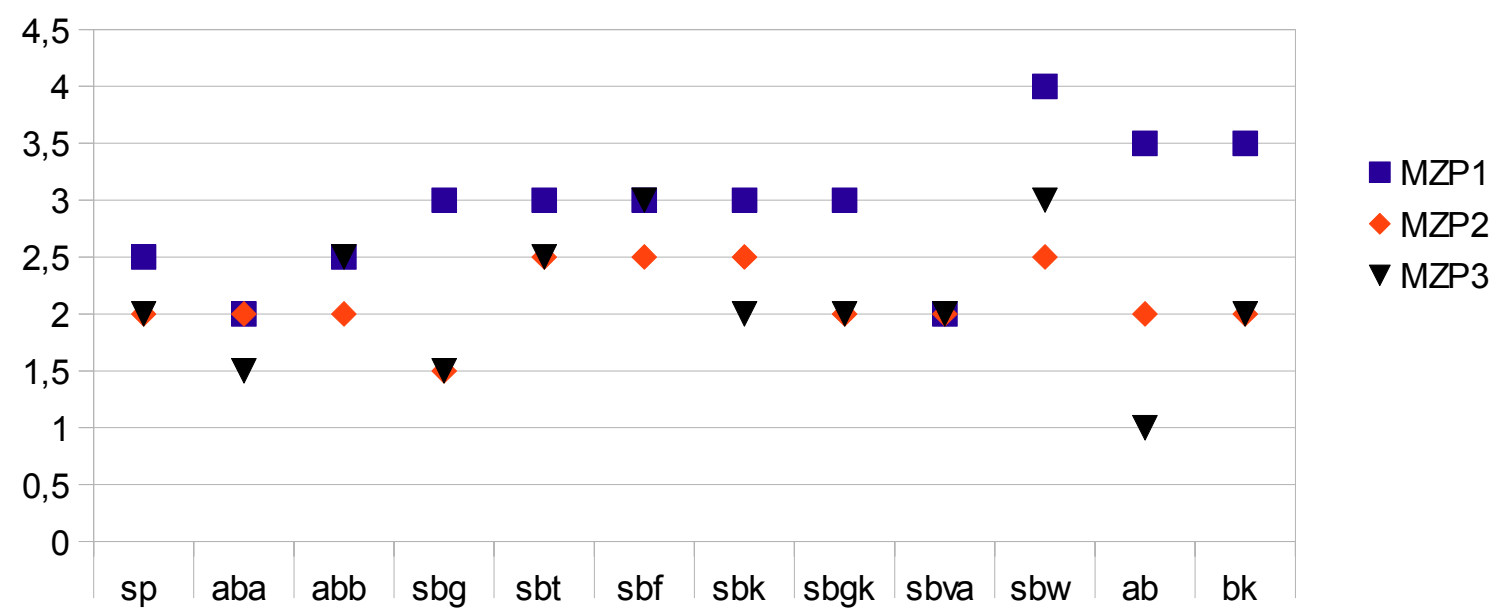

Abbildung 46: Entwicklung der Tennistechnik über 3 Messzeitpunkte bei Kind NV 


\section{Bewegungslernen}

Kind $\mathrm{PH}$

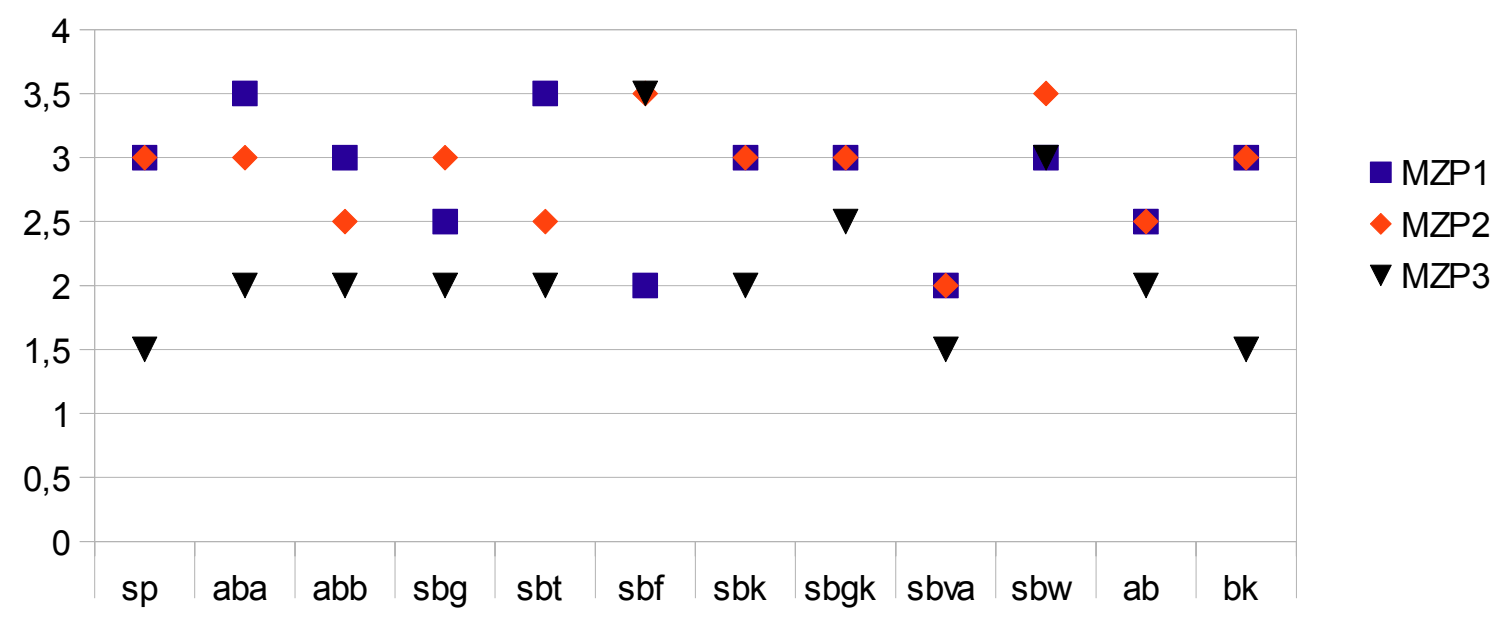

Abbildung 47: Entwicklung der Tennistechnik über 3 Messzeitpunkte bei Kind PH

\section{Bewegungslernen}

Kind SK

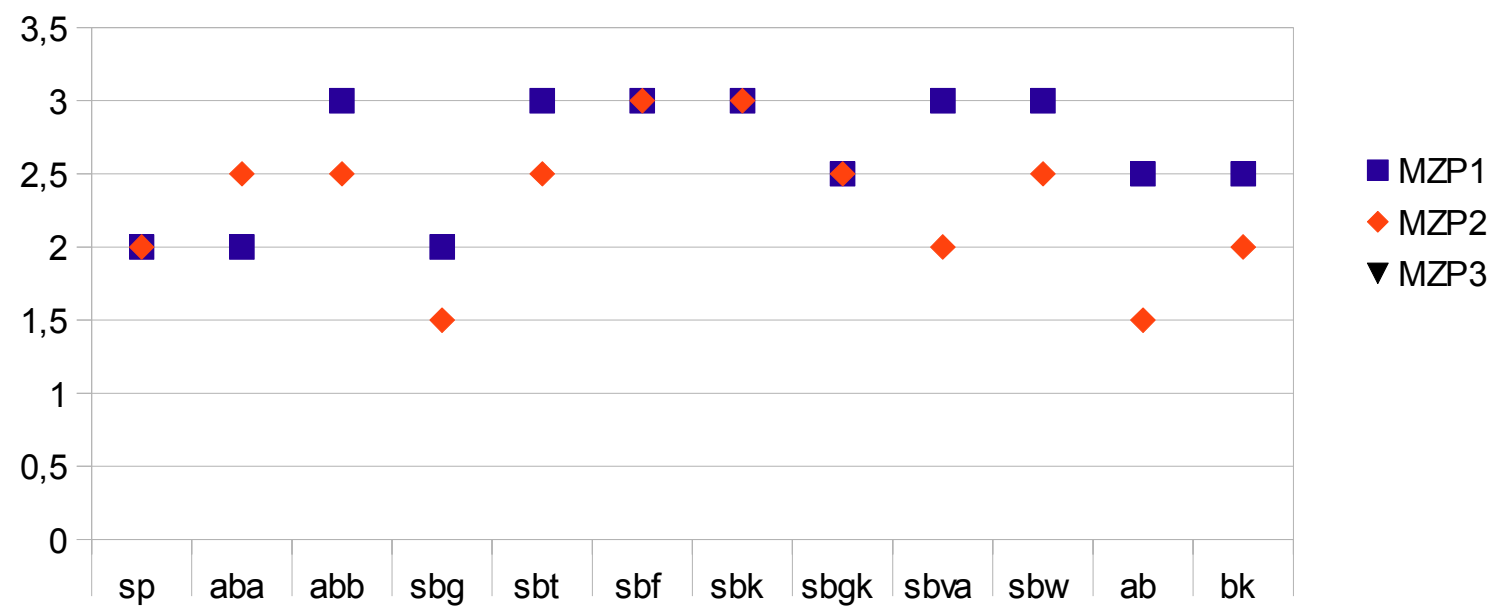

Abbildung 48: Entwicklung der Tennistechnik über 3 Messzeitpunkte bei Kind SK 


\section{Bewegungslernen}

Kind SL

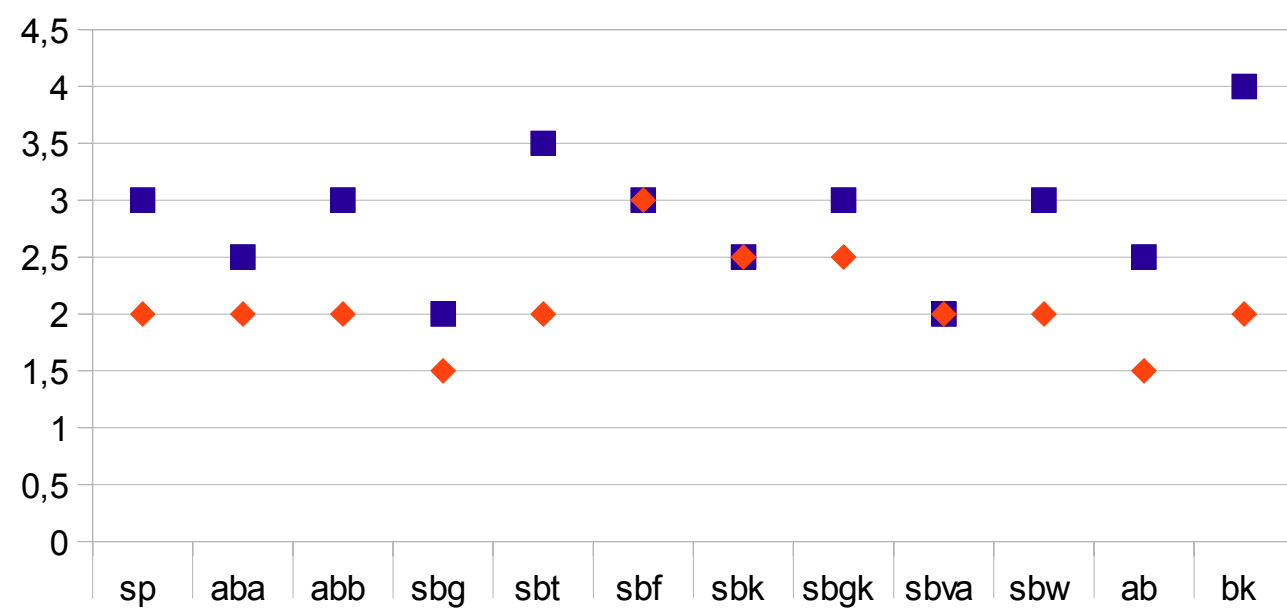

Abbildung 49: Entwicklung der Tennistechnik über 3 Messzeitpunkte bei Kind SL

\section{Bewegungslernen}

Kind SP

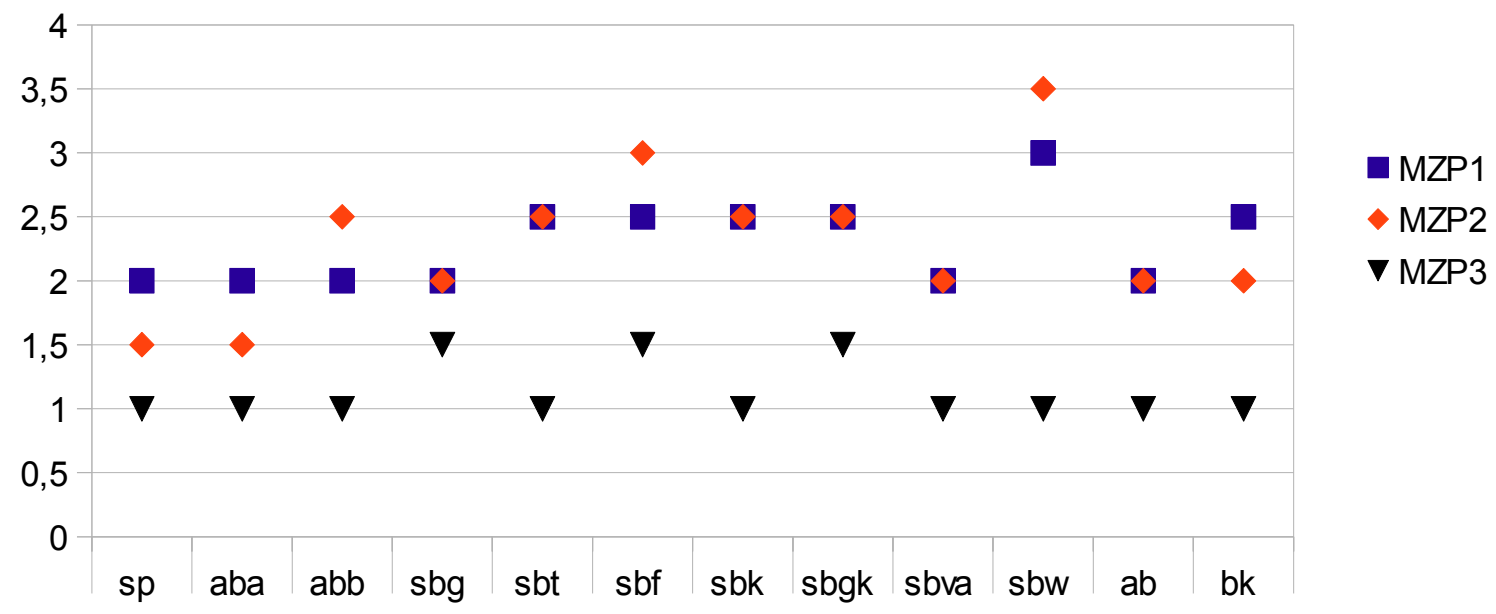

Abbildung 50: Entwicklung der Tennistechnik über 3 Messzeitpunkte bei Kind SP 


\section{Bewegungslernen}

\section{Kind TB}

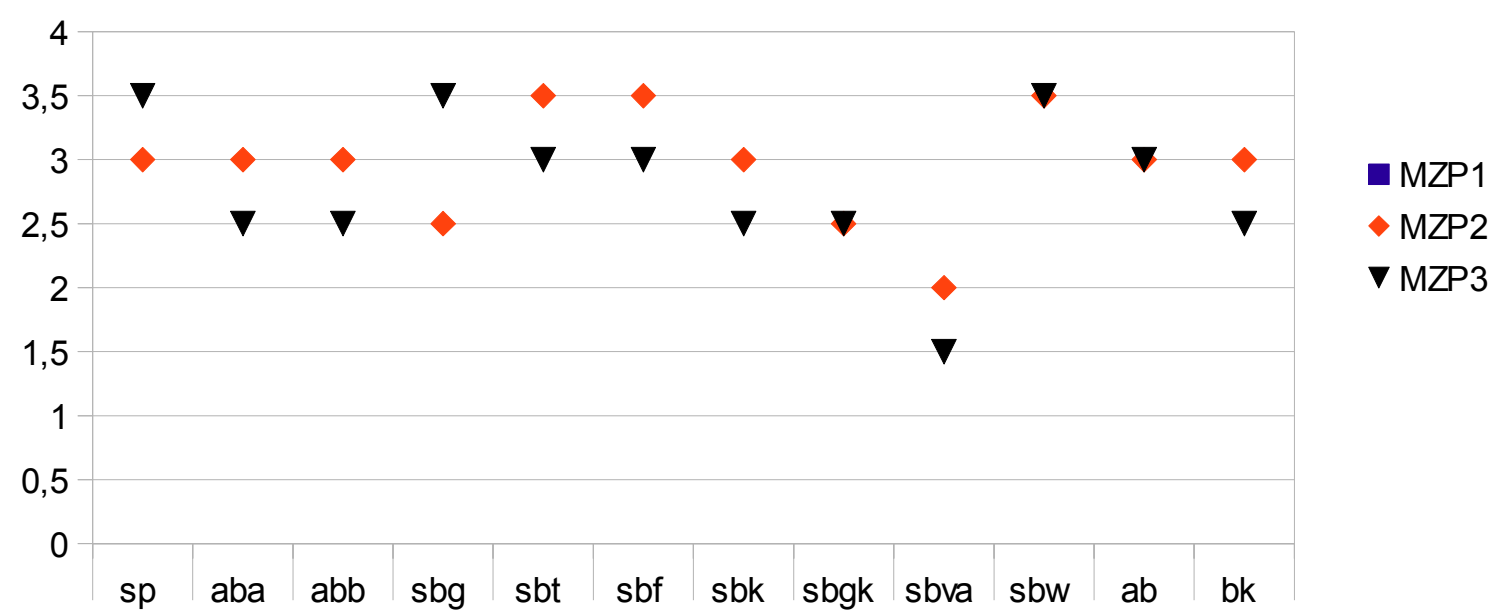

Abbildung 51: Entwicklung der Tennistechnik über 3 Messzeitpunkte bei Kind TB

\section{Bewegungslernen}

Kind TH

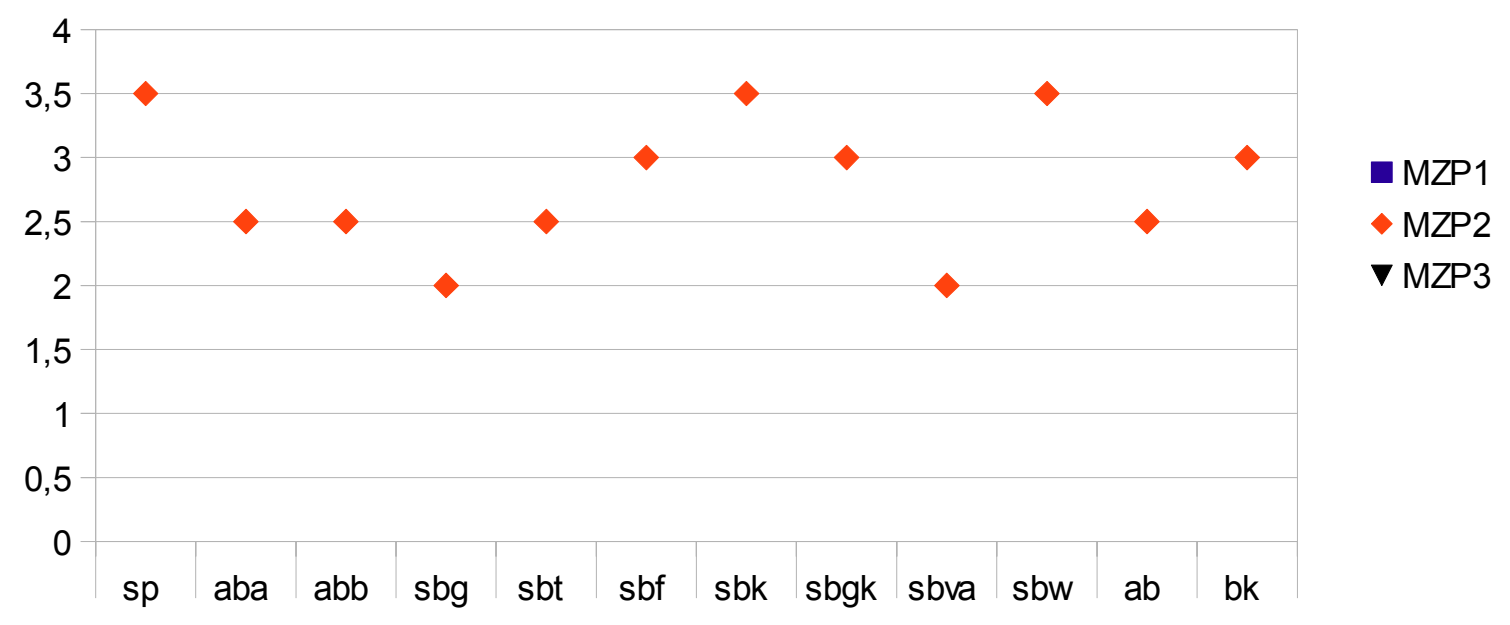

Abbildung 52: Entwicklung der Tennistechnik über 3 Messzeitpunkte bei Kind TH 


\subsection{Rohdaten Bewegungslernen}

Es werden die Rohdaten zum Thema Bewegungslernen visualisiert. Dies geschieht pro Merkmal pro Messzeitpunkt. Die Messzeitpunkte über der Mittelwertline geben unterdurchschnittliche Werte an. Demzufolge sind überdurchschnittliche Werte unter der Mittelwertlinie angegeben. „MS“ des ersten Kindes entspricht „MAS“. 


\section{Bewegungslernen}

Merkmal SP1

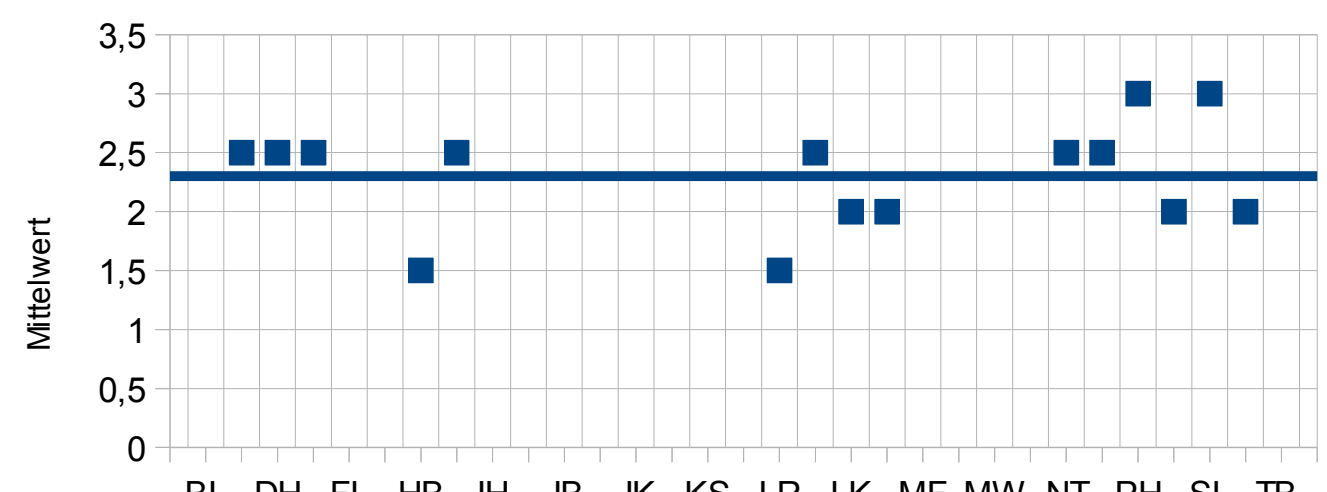

BL DH FL HB IH JB JK KS LR LK MF MW NT PH SL TB $A B$ BT FB GK HW JS JE KB LH LW MS MS NM NV SK SOP TH

Kind

Abbildung 53: Tennismerkmal SP1 bei den Kindern

\section{Bewegungslernen}

Merkmal SP2

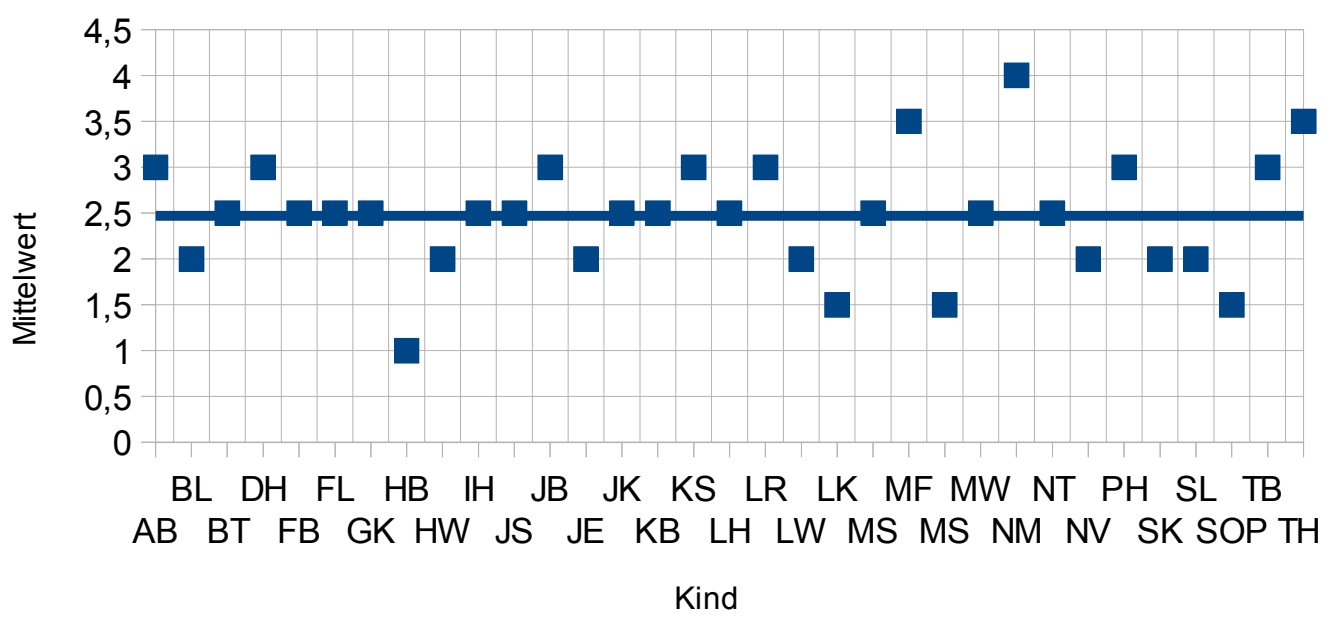

Abbildung 54: Tennismerkmal SP2 bei den Kindern 


\section{Bewegungslernen}

Merkmal SP3

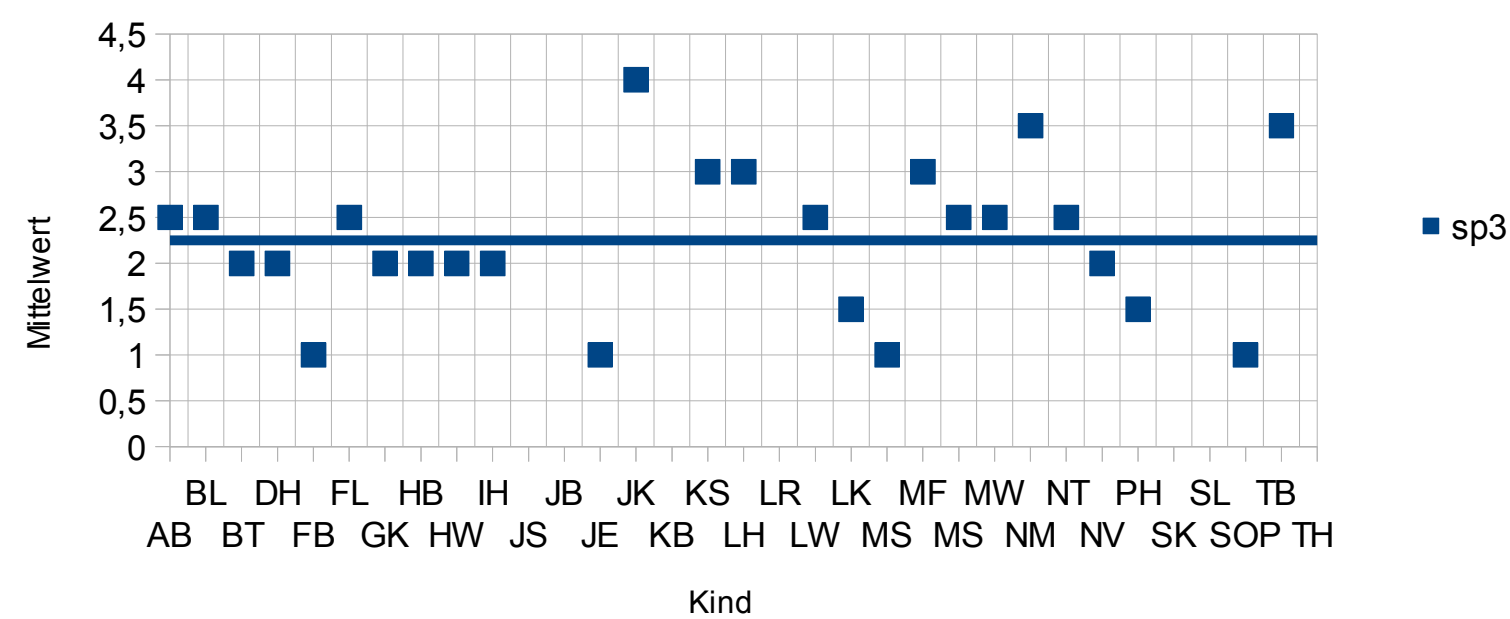

Abbildung 55: Tennismerkmal SP3 bei den Kindern

\section{Bewegungslernen}

Merkmal ABA1

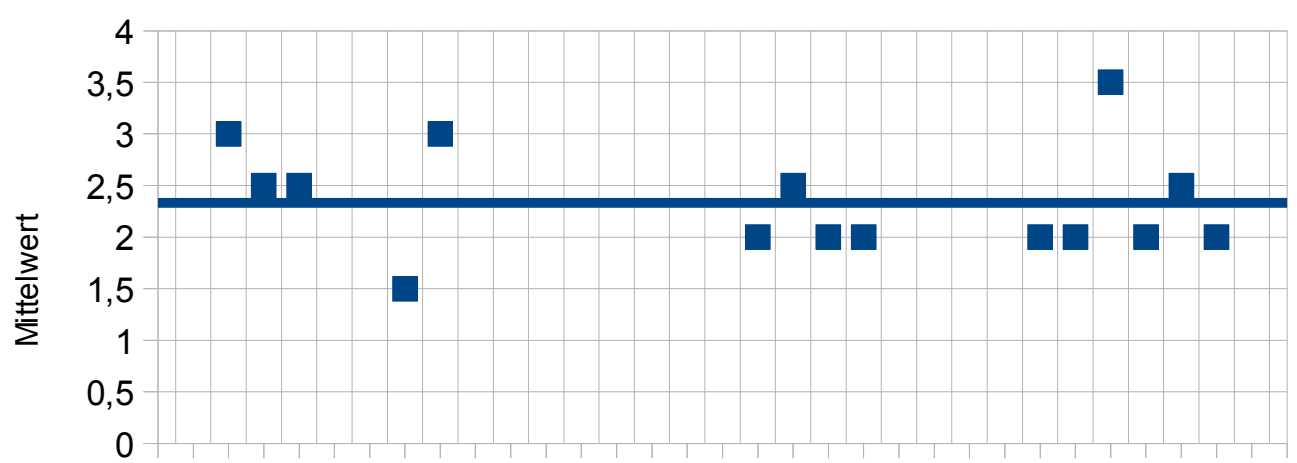

BL DH FL HB IH JB JK KS LR LK MF MW NT PH SL TB AB BT FB GK HW JS JE KB LH LW MS MS NM NV SK SOP TH Kind

Abbildung 56: Tennismerkmal ABA1 bei den Kindern 


\section{Bewegungslernen}

\section{Merkmal ABA2}

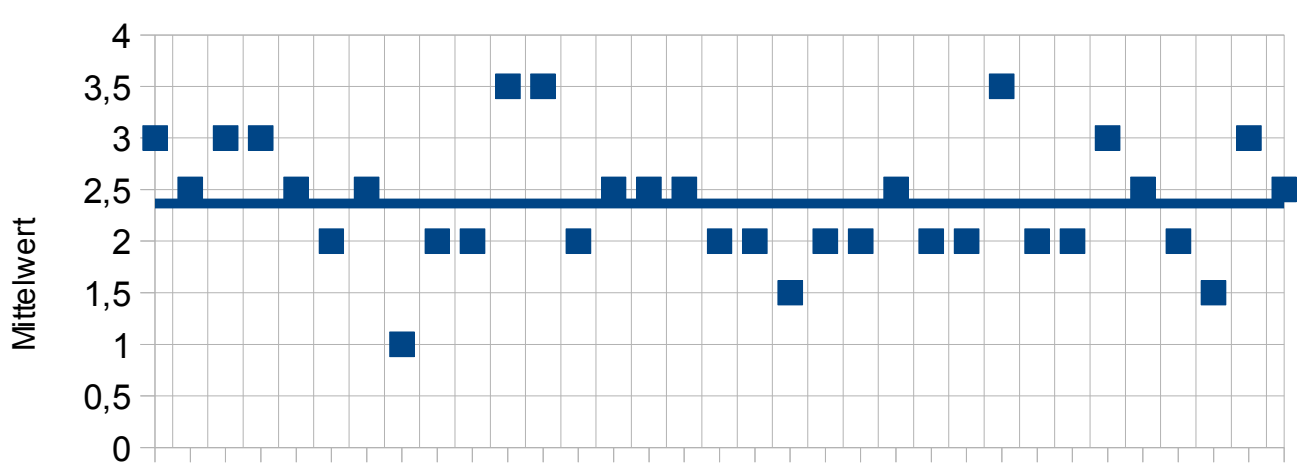

BL DH FL HB IH JB JK KS LR LK MF MW NT PH SL TB AB BT FB GK HW JS JE KB LH LW MS MS NM NV SK SOP TH

Kind

Abbildung 57: Tennismerkmal ABA2 bei den Kindern

\section{Bewegungslernen}

\section{Merkmal ABA3}

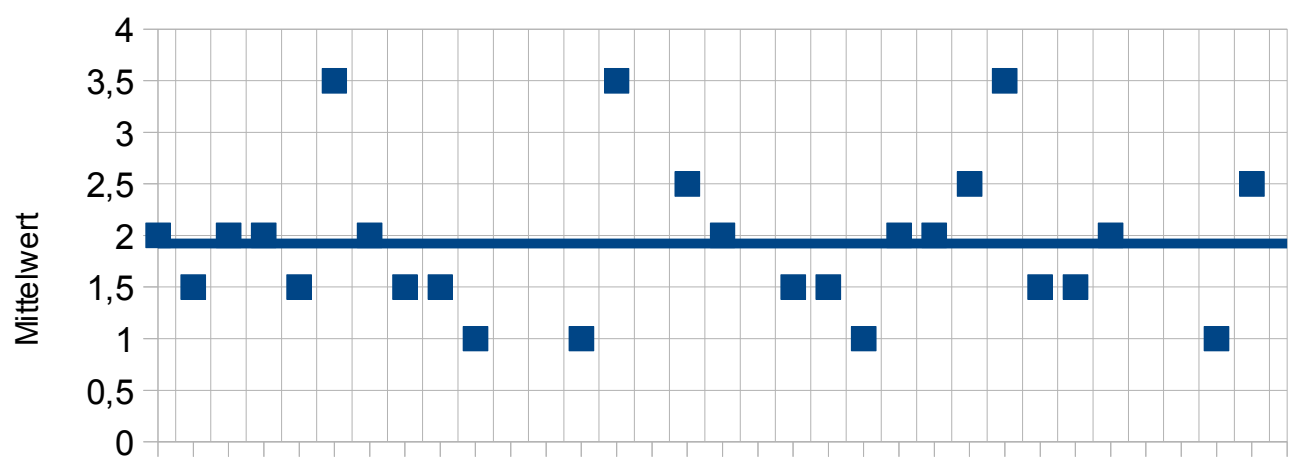

BL DH FL HB IH JB JK KS LR LK MF MW NT PH SL TB AB BT FB GK HW JS JE KB LH LW MS MS NM NV SK SOP TH

Kind

Abbildung 58: Tennismerkmal ABA3 bei den Kindern 


\section{Bewegungslernen}

\section{Merkmal ABB1}

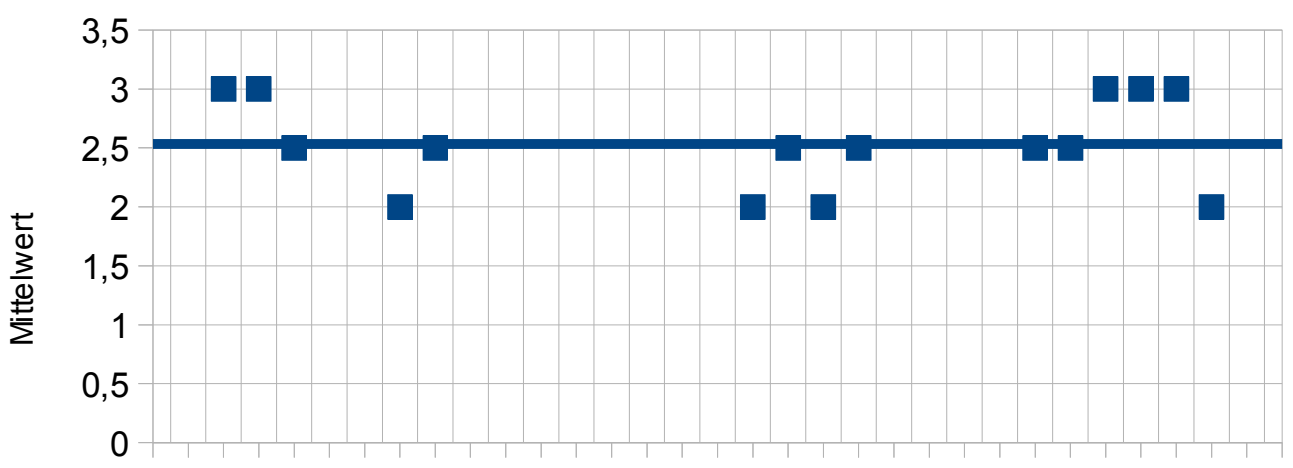

BL DH FL HB IH JB JK KS LR LK MF MW NT PH SL TB AB BT FB GK HW JS JE KB LH LW MS MS NM NV SK SOP TH

Kind

Abbildung 59: Tennismerkmal ABB1 bei den Kindern

\section{Bewegungslernen}

\section{Merkmal ABB2}

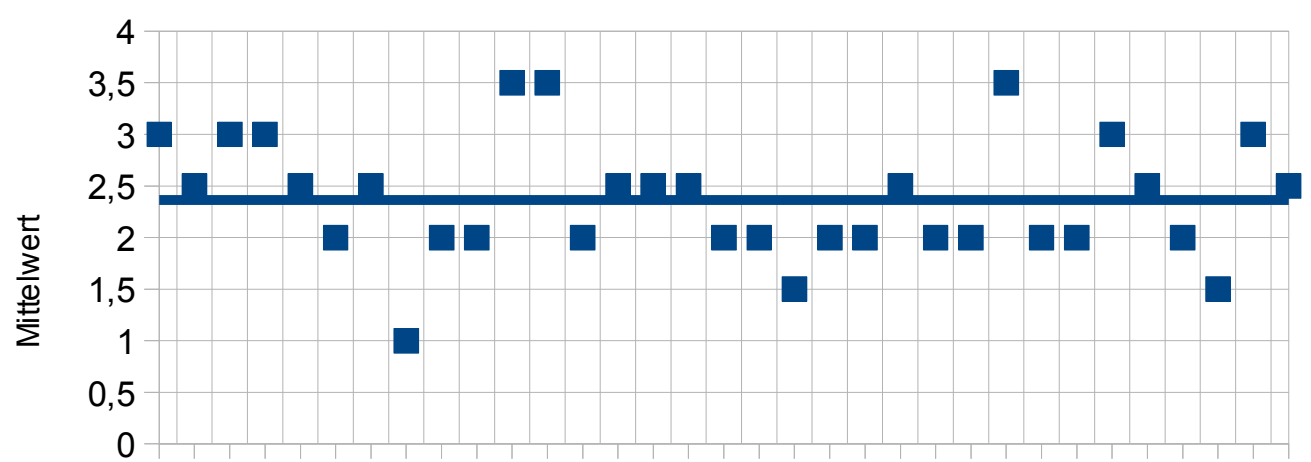

BL DH FL HB IH JB JK KS LR LK MF MW NT PH SL TB AB BT FB GK HW JS JE KB LH LW MS MS NM NV SK SOP TH Kind 


\section{Bewegungslernen}

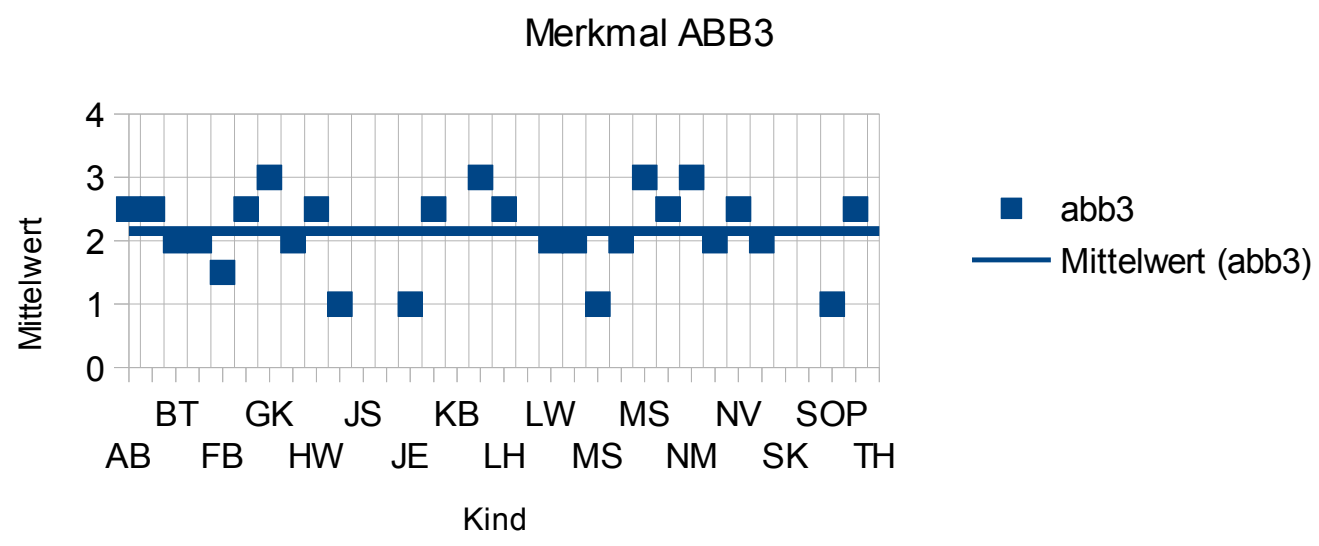

Abbildung 61: Tennismerkmal ABB3 bei den Kindern

\section{Bewegungslernen}

Merkmal SBG1

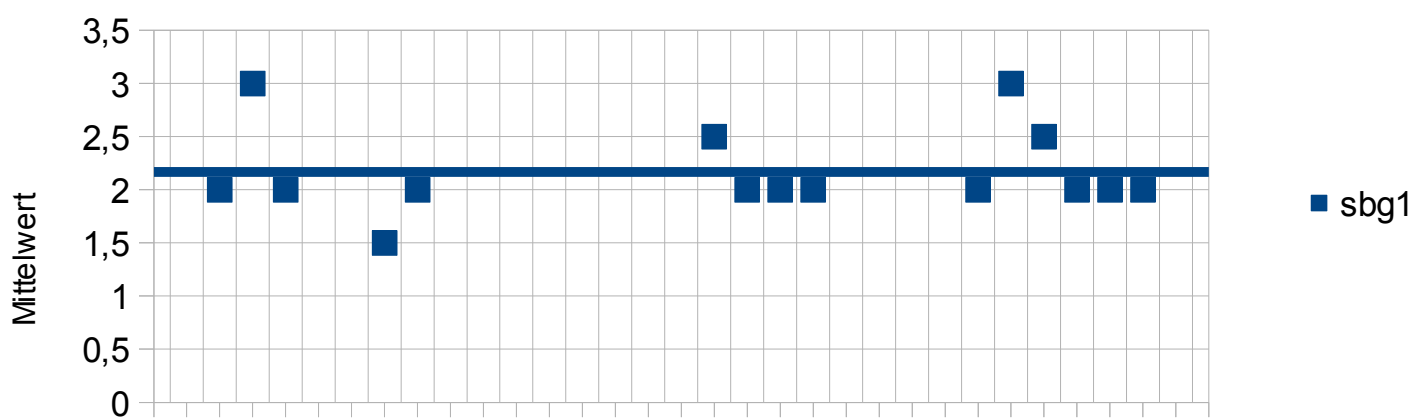

BL DH FL HB IH JB JK KS LR LK MF MW NT PH SL TB AB BT FB GK HW JS JE KB LH LW MS MS NM NV SKSOPTH

Kind

Abbildung 62: Tennismerkmal SBG1 bei den Kindern 


\section{Bewegungslernen}

\section{Merkmal SBG2}

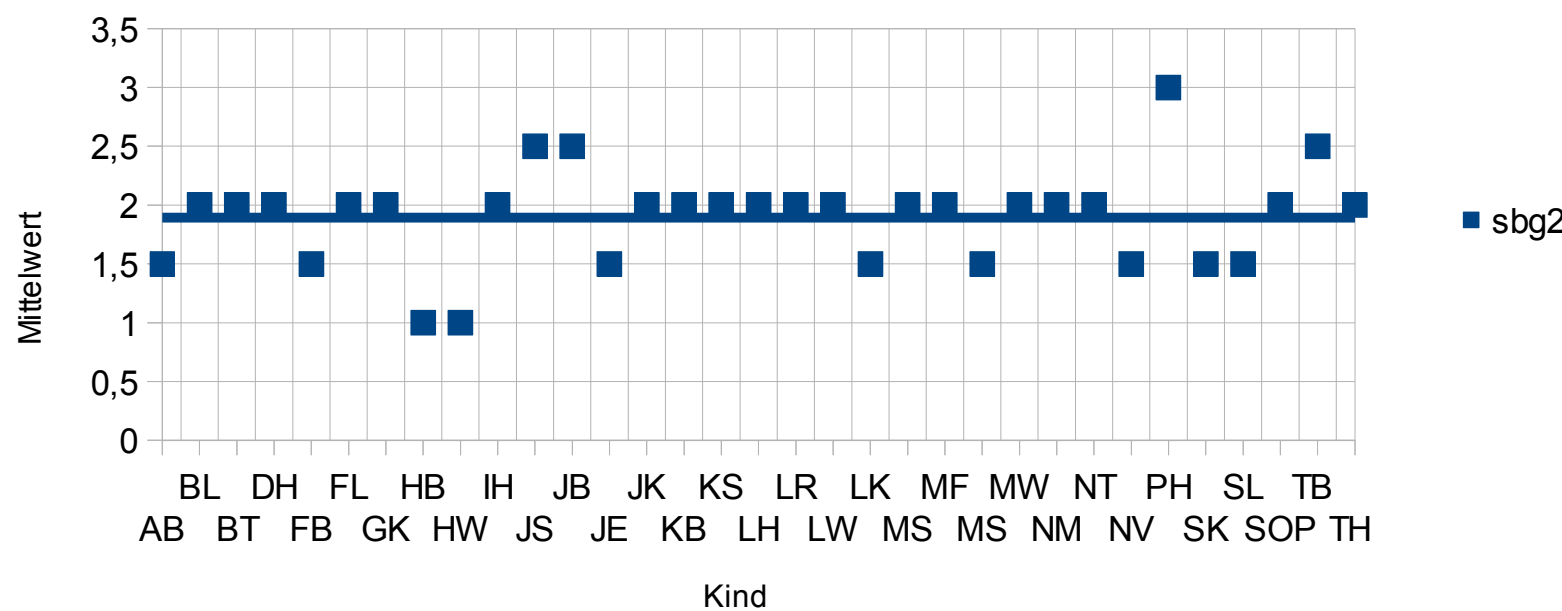

Abbildung 63: Tennismerkmal SBG2 bei den Kindern

\section{Bewegungslernen}

Merkmal SBG3

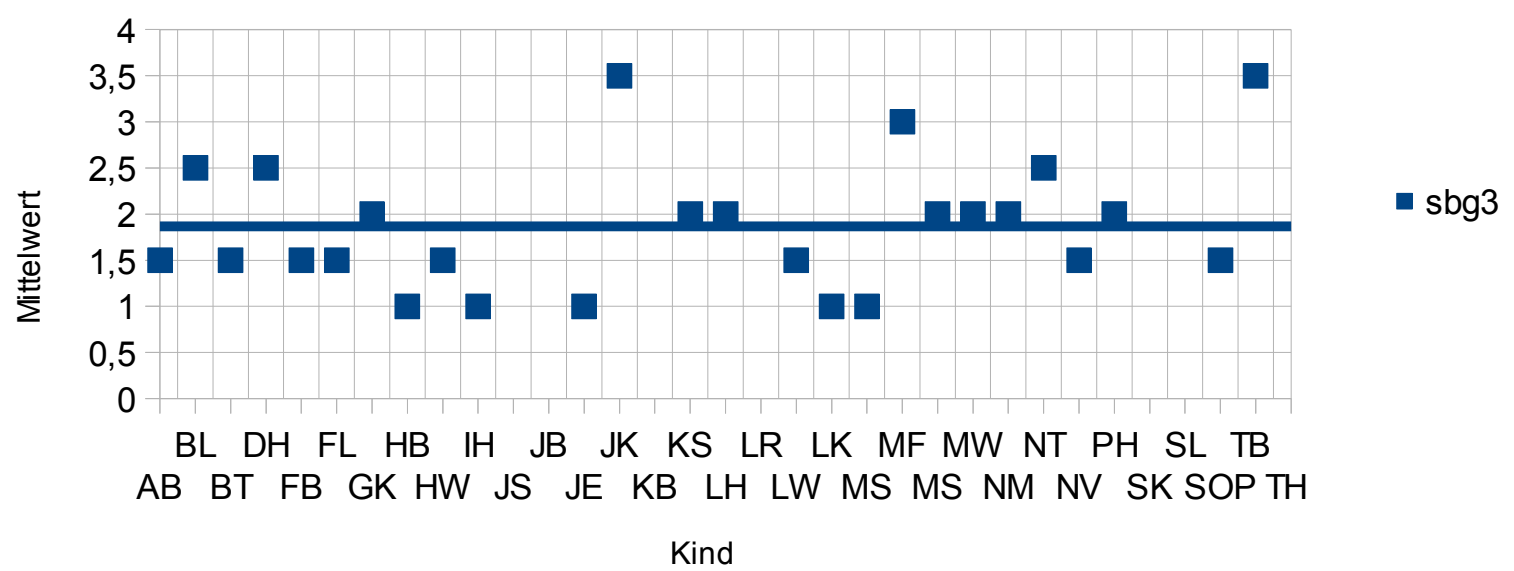

Abbildung 64: Tennismerkmal SBG3 bei den Kindern 


\section{Bewegungslernen}

Merkmal SBT1

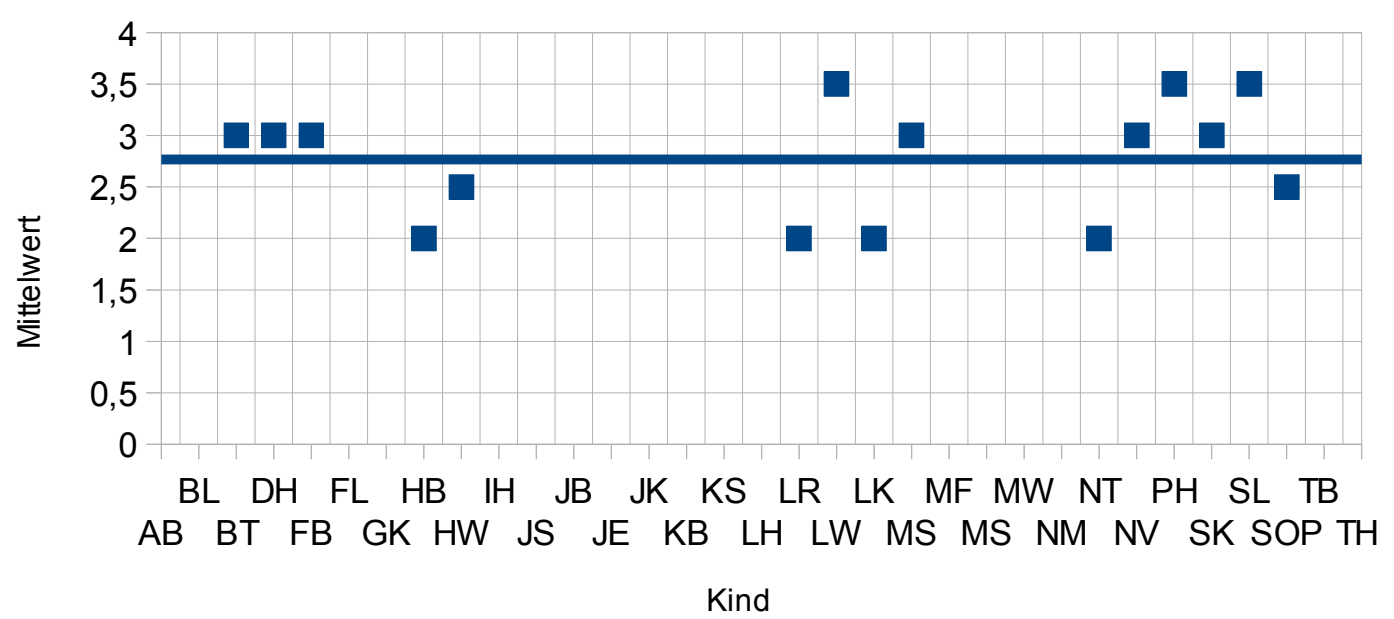

Abbildung 65: Tennismerkmal SBT1 bei den Kindern

\section{Bewegungslernen}

Merkmal SBT2

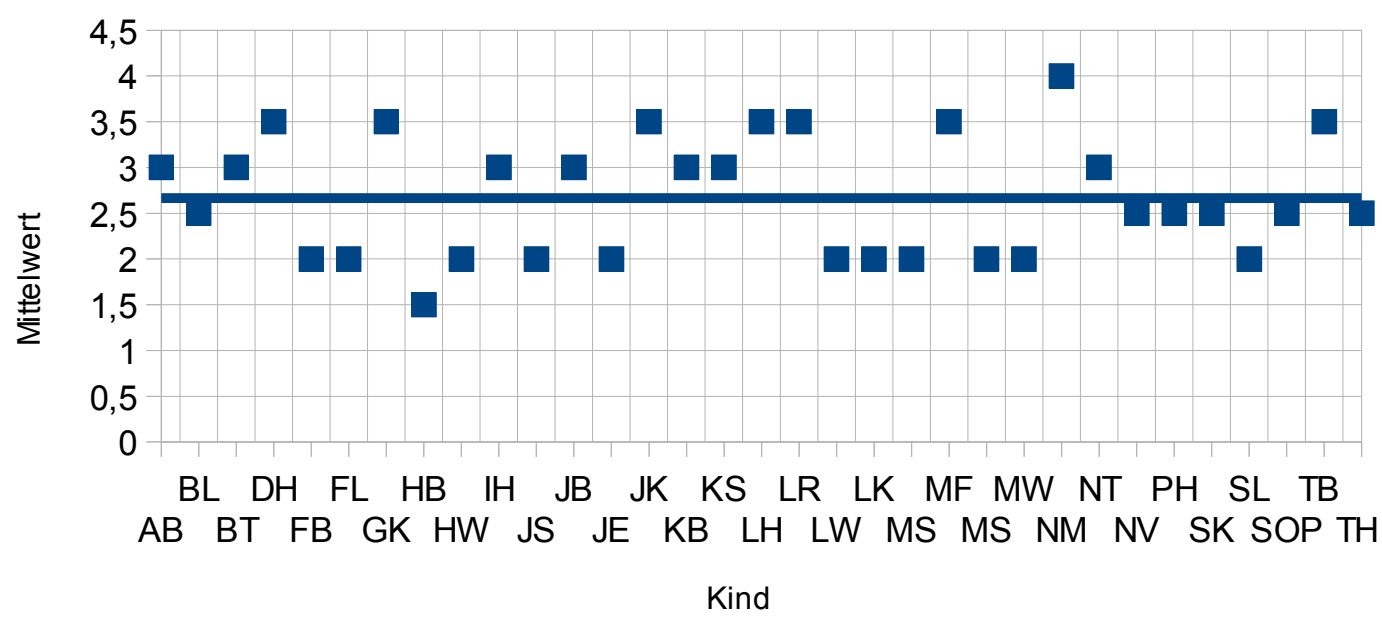

Abbildung 66: Tennismerkmal SBT2 bei den Kindern 


\section{Bewegungslernen}

\section{Merkmal SBT3}

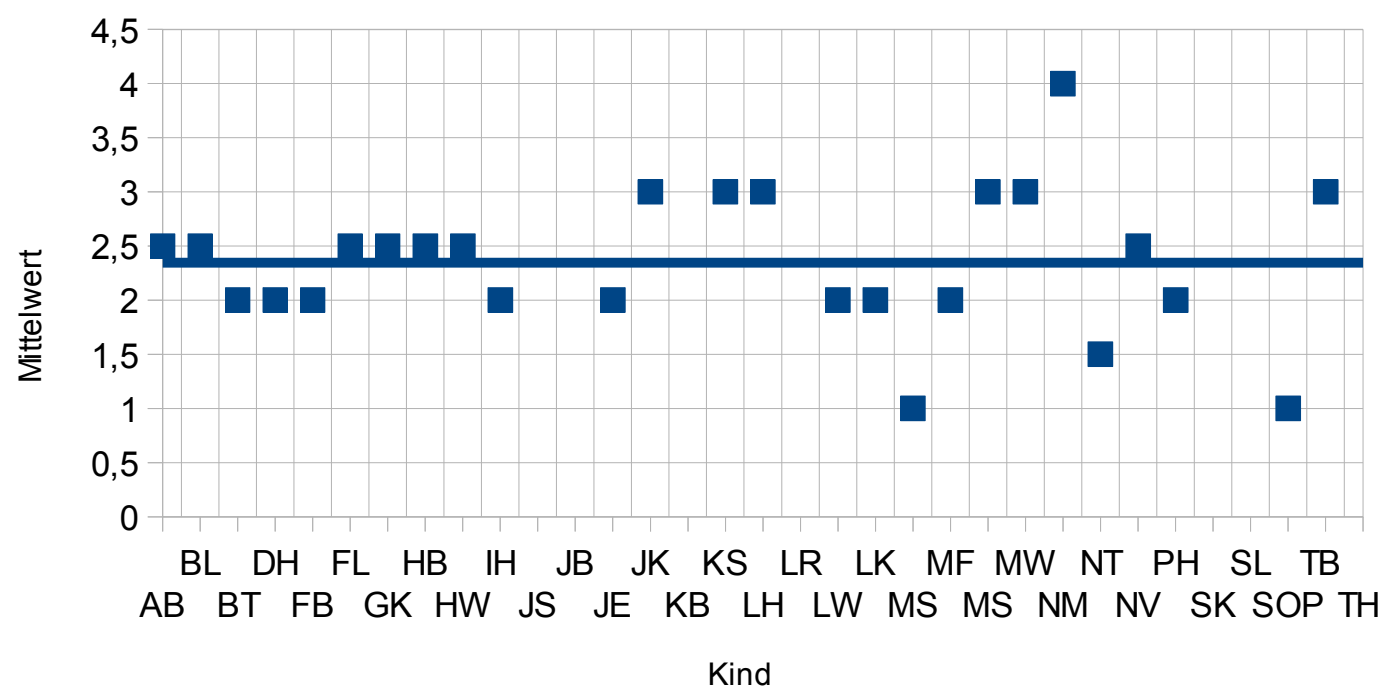

Abbildung 67: Tennismerkmal SBT3 bei den Kindern

\section{Bewegungslernen \\ Merkmal SBF1}

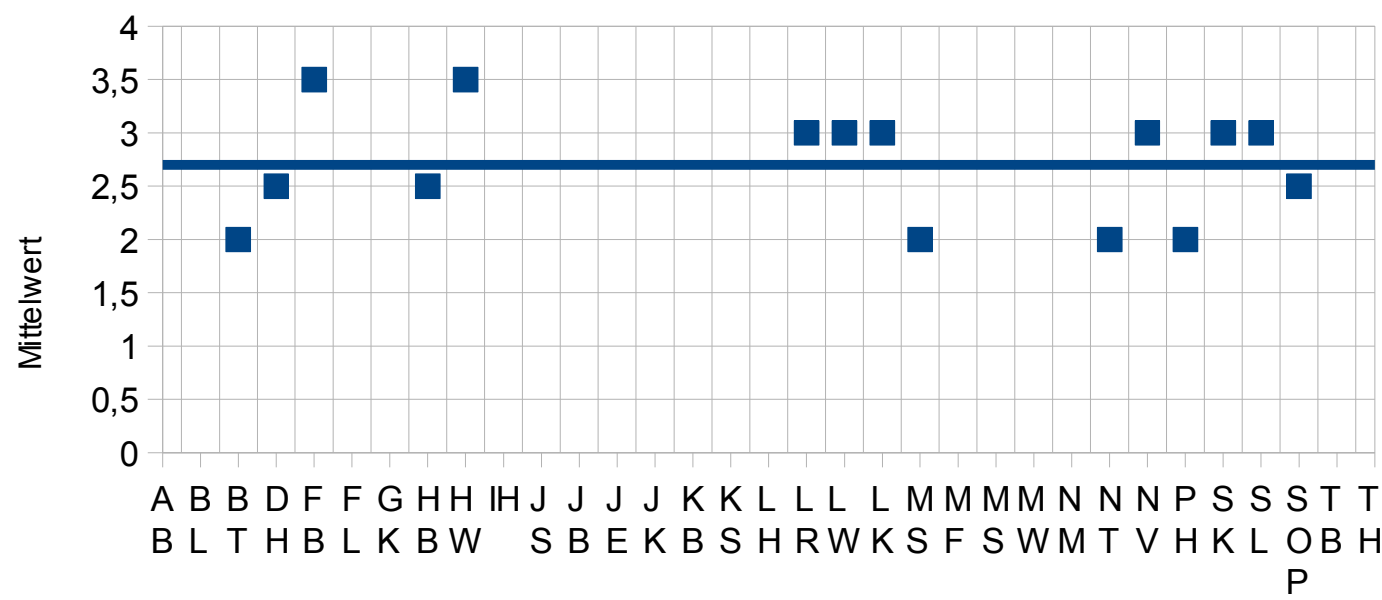

Kind

Abbildung 68: Tennismerkmal SBF1 bei den Kindern 
Bewegungslernen Merkmal SBF2

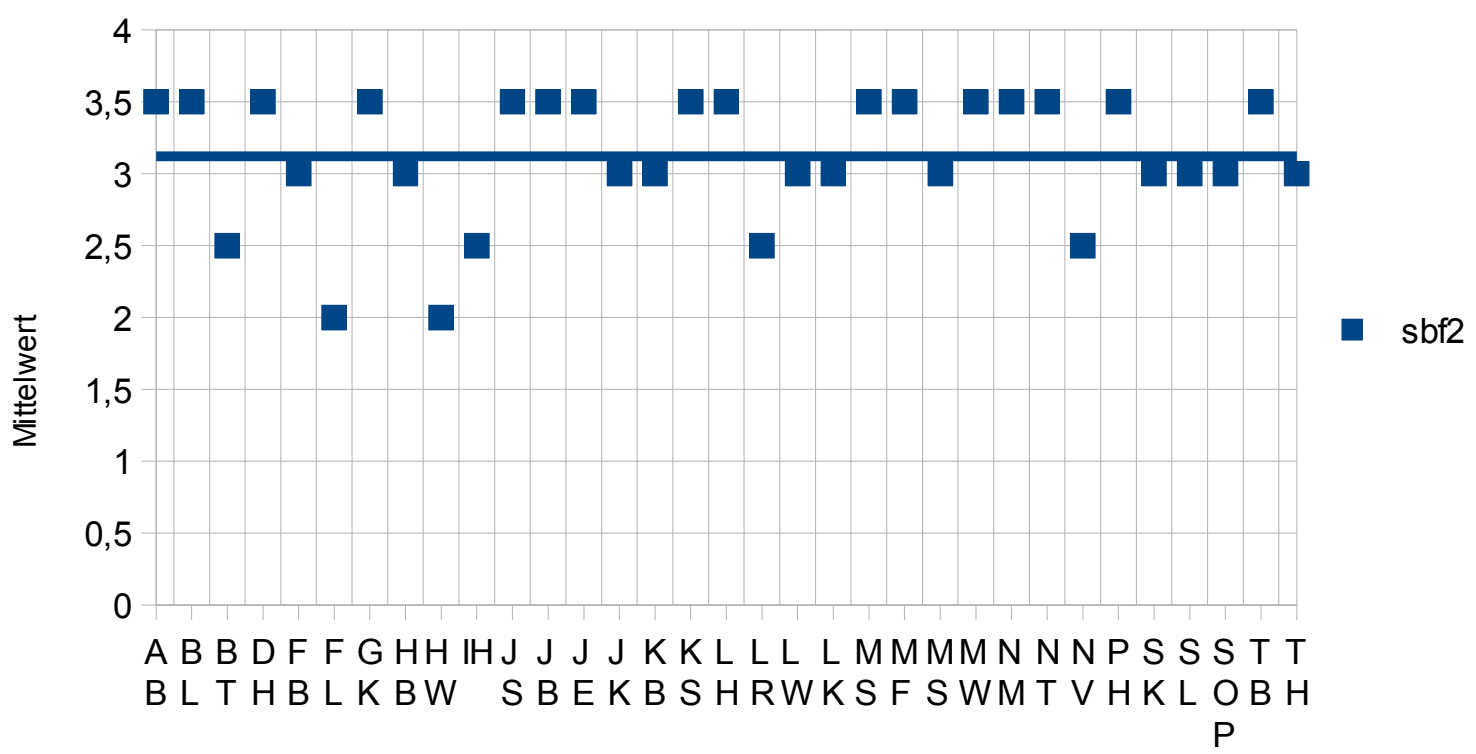

Kind

Abbildung 69: Tennismerkmal SBF2 bei den Kindern

Bewegungslernen Merkmal SBF3

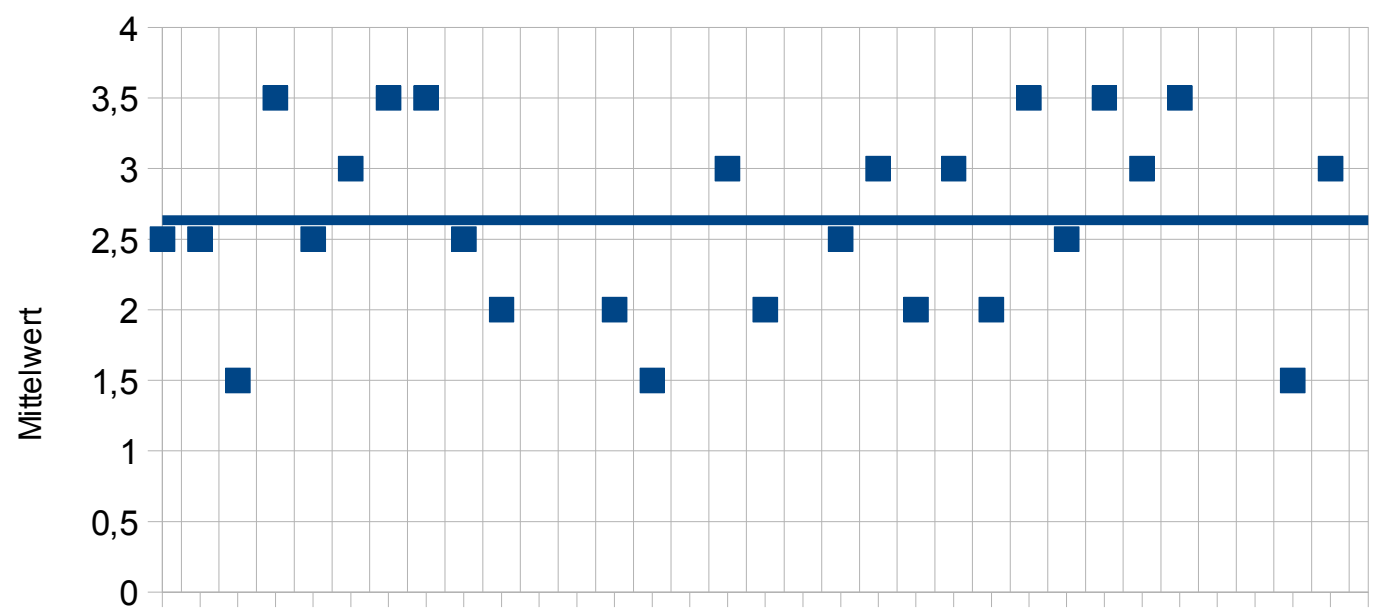

A B B DFFGHHIHJJ J JKKLLLLMMMMNNNPSSST T BLTHBLKBW SBEKBSHRWKSF SWMTVHKLOBH $\mathrm{P}$

Kind

Abbildung 70: Tennismerkmal SBF3 bei den Kindern 
Bewegungslernen Merkmal SBK1

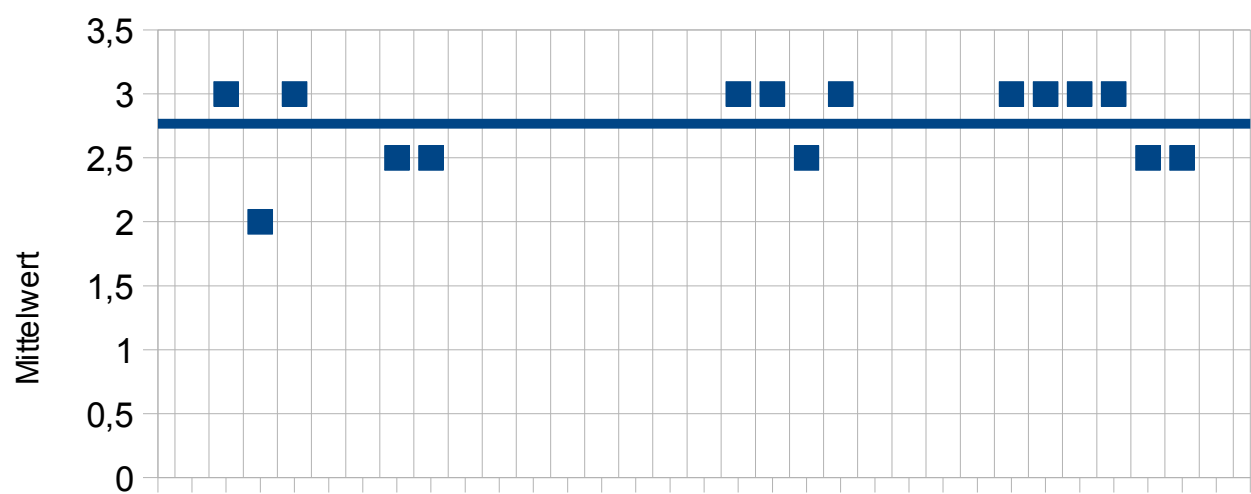

sbk1

A B B D F F GHH IHJ J J J KKLLL LMMMMN NNPSSST T BLTHBLKBW SBEKBSHRWKSF SWMTVHKLOBH

Kind

Abbildung 71: Tennismerkmal SBK1 bei den Kindern

Bewegungslernen Merkmal SBK2

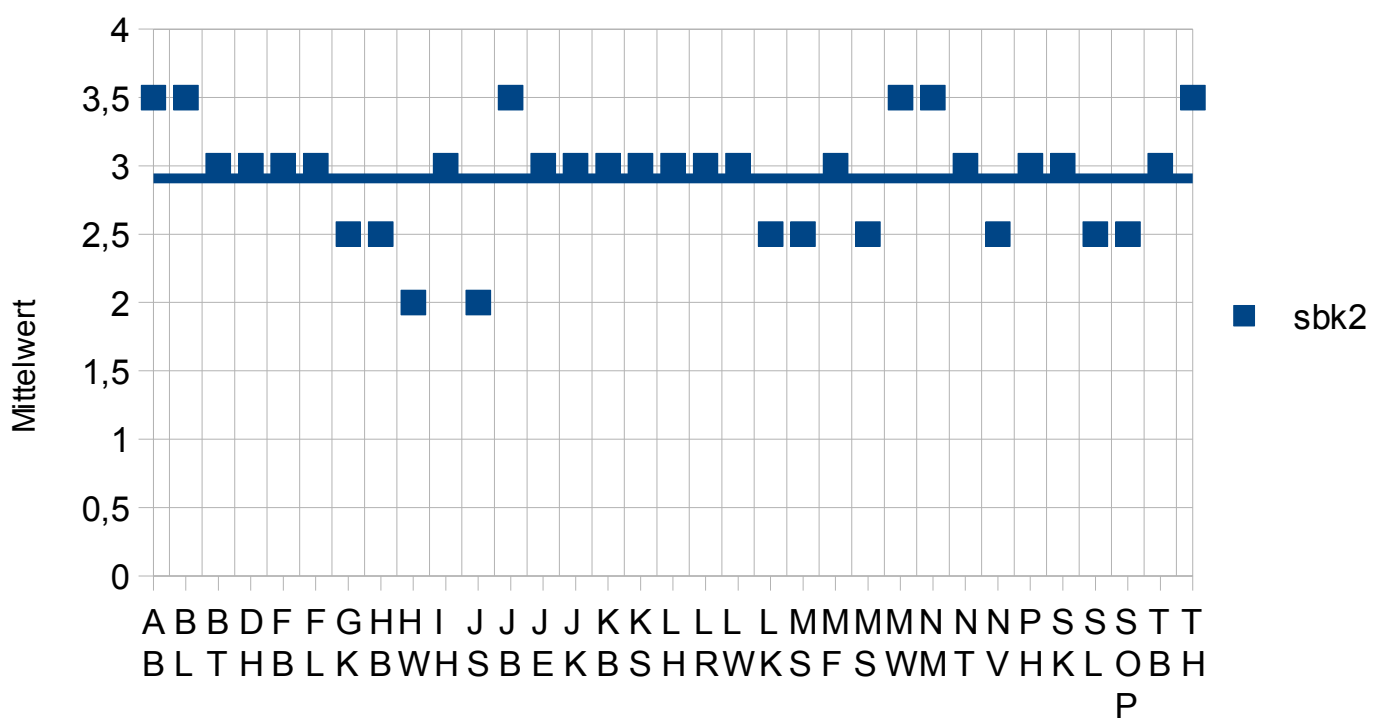

Kind

Abbildung 72: Tennismerkmal SBK2 bei den Kindern 


\section{Bewegungslernen Merkmal SBK3}

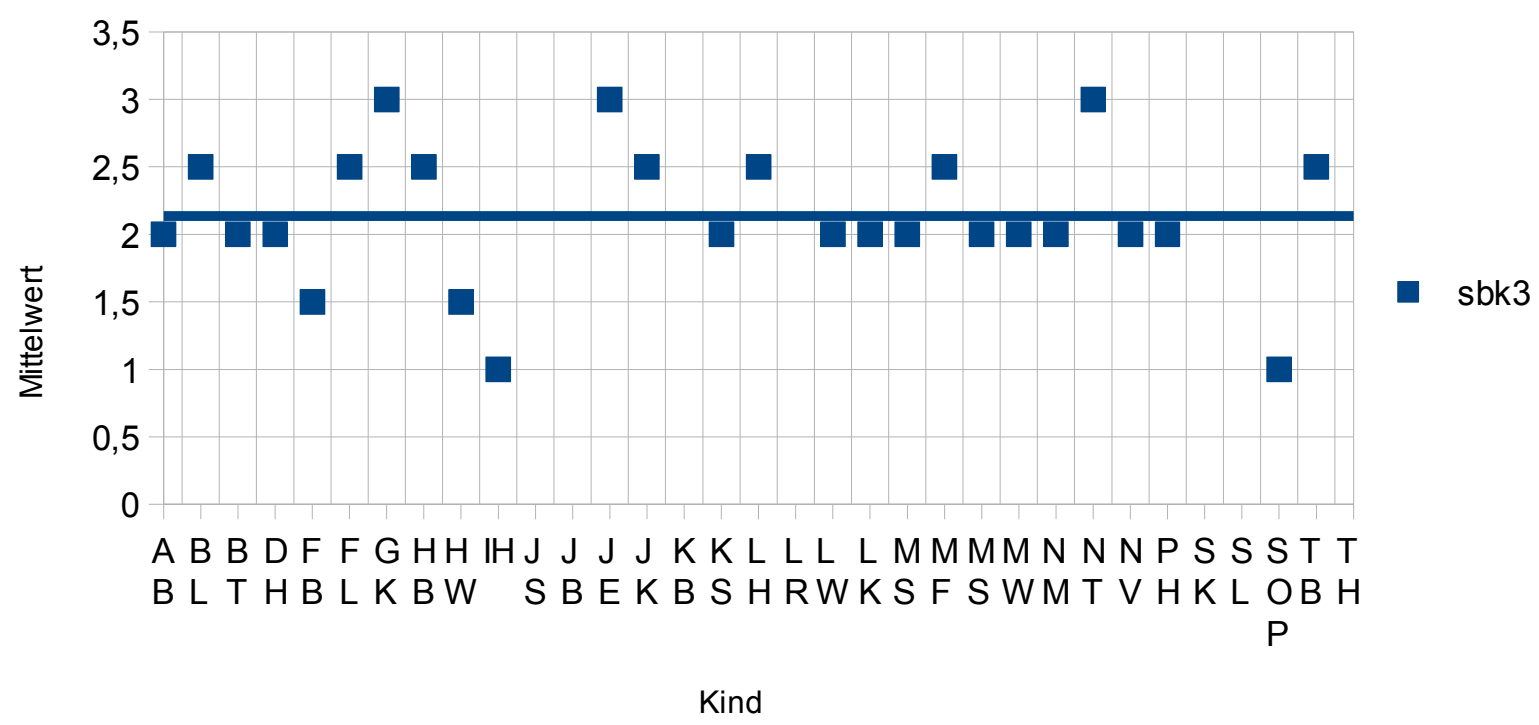

Abbildung 73: Tennismerkmal SBK3 bei den Kindern

Bewegungslernen

Merkmal SBGK1

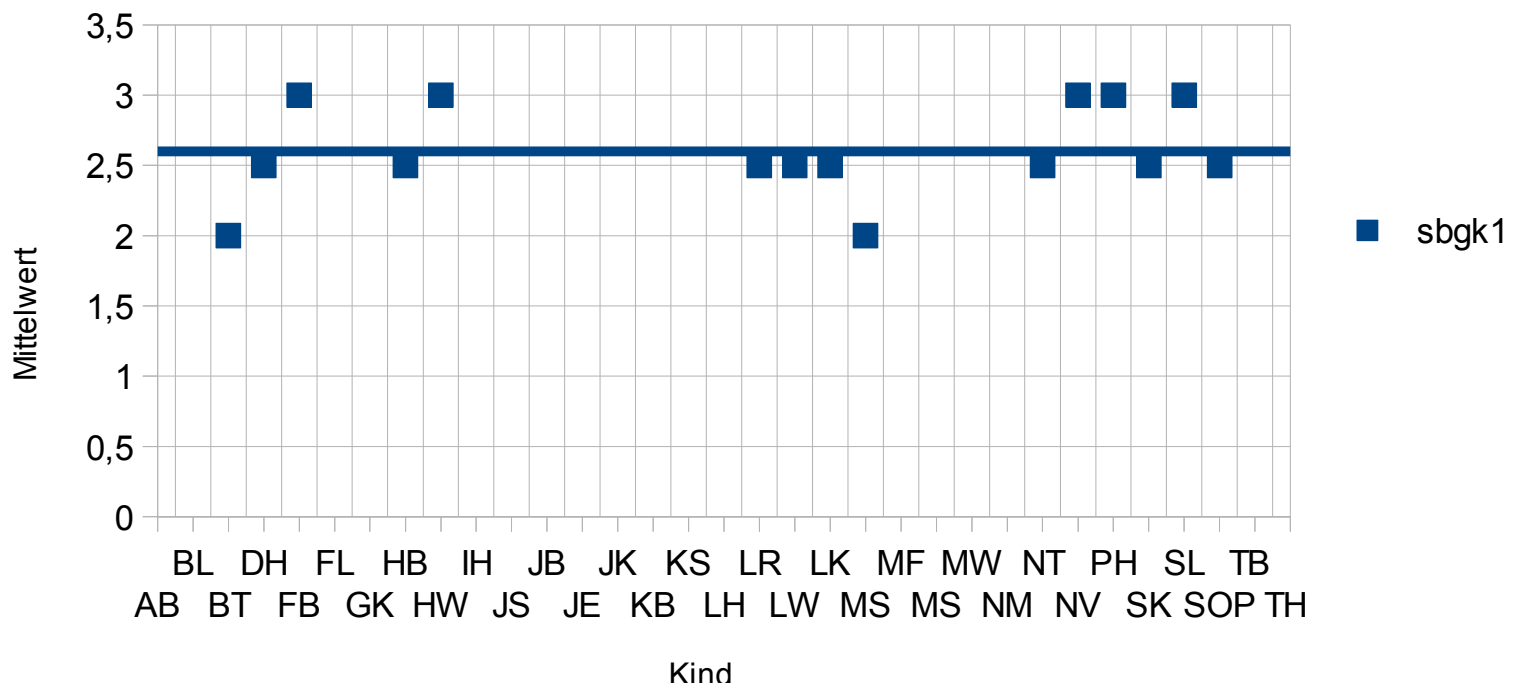

Abbildung 74: Tennismerkmal SBGK1 bei den Kindern 


\section{Bewegungslernen}

Merkmal SBGK2

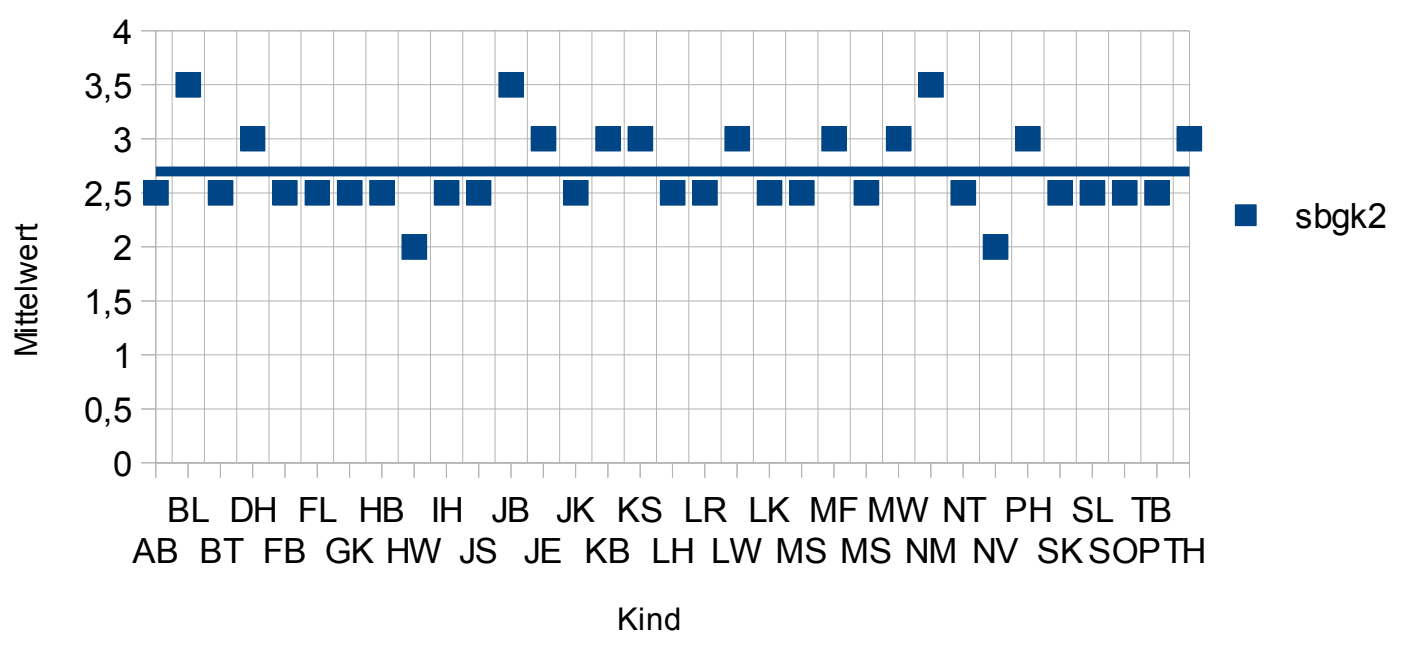

Abbildung 75: Tennismerkmal SBGK2 bei den Kindern 


\section{Bewegungslernen}

\section{Merkmal SBGK3}

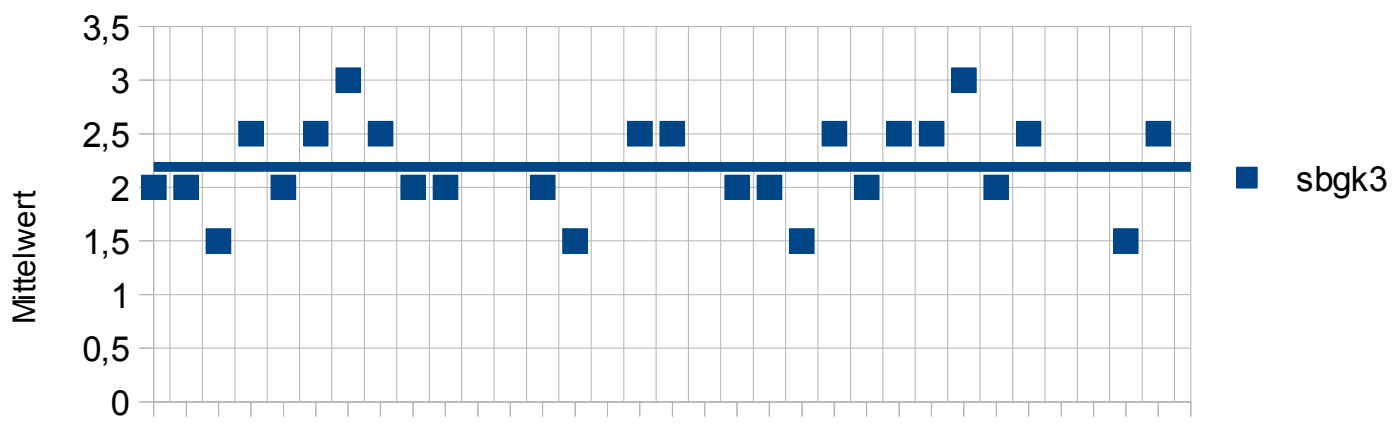

BL DH FL HB IH JB JK KS LR LK MF MW NT PH SL TB AB BT FB GK HW JS JE KB LH LW MS MS NM NV SKSOPTH

Kind

Abbildung 76: Tennismerkmal SBGK3 bei den Kindern

\section{Bewegungslernen}

Merkmal SBVA1

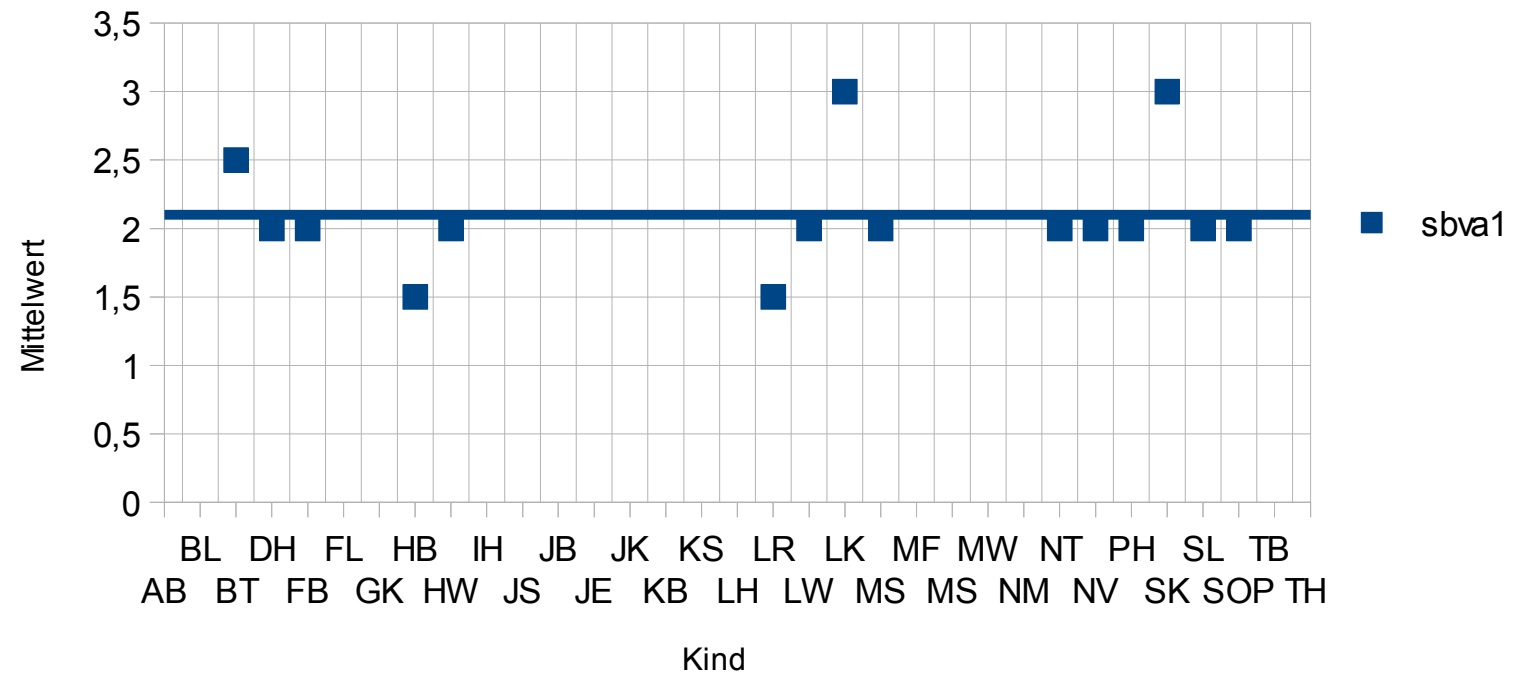

Abbildung 77: Tennismerkmal SBVA1 bei den Kindern 
Merkmal SBVA3

\section{Bewegungslernen}

\section{Merkmal SBVA2}

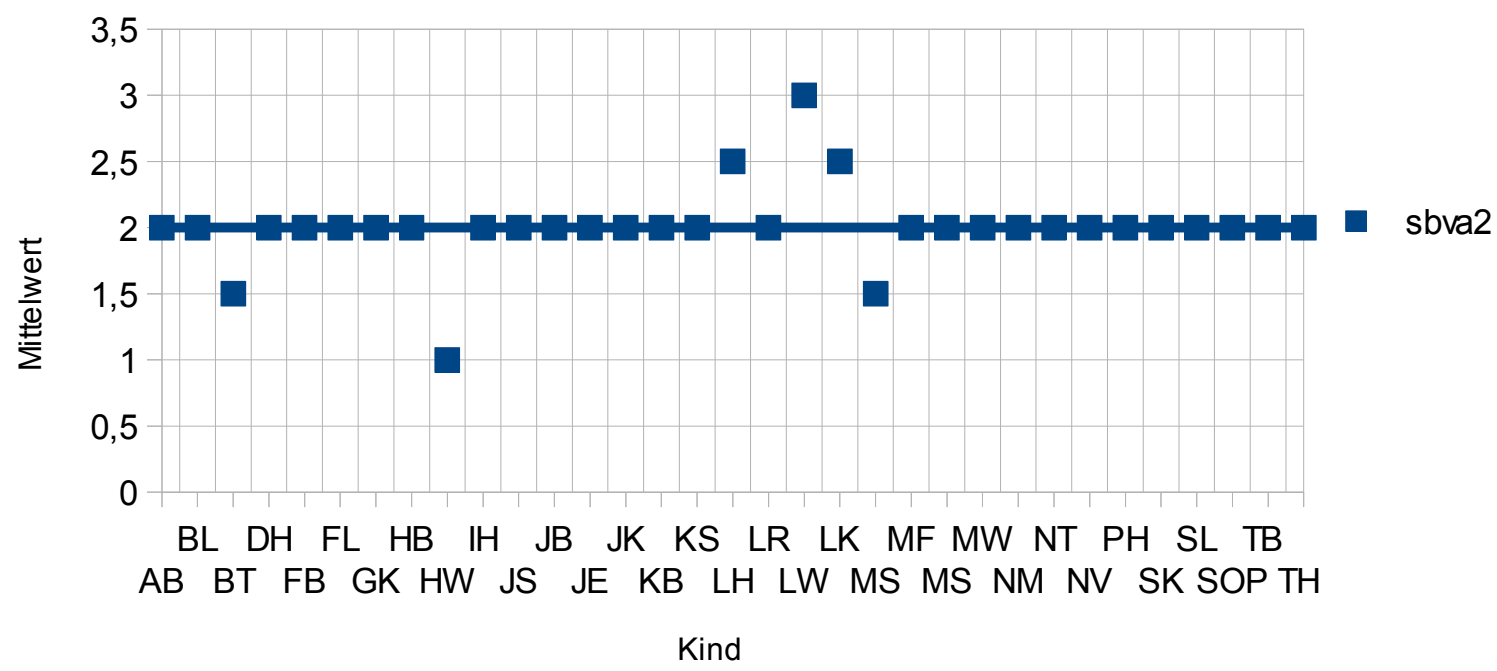

Abbildung 79: Tennismerkmal SBVA2 bei den Kindern

\section{Bewegungslernen}

Merkmal SBW1

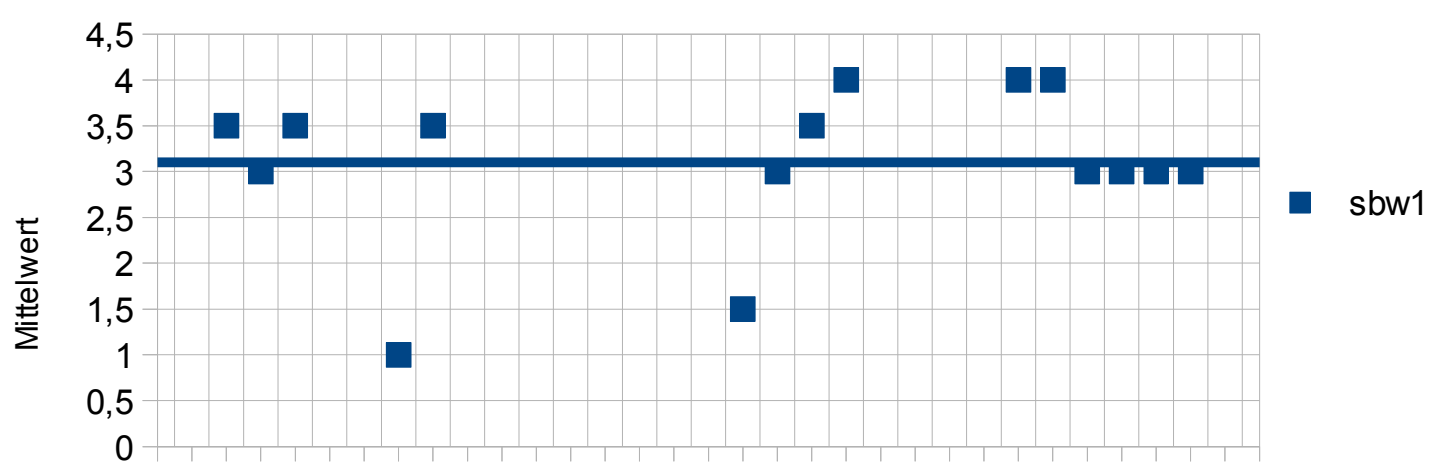

BL DH FL HB IH JB JK KS LR LK MF MW NT PH SL TB AB BT FB GK HW JS JE KB LH LW MS MS NM NV SK SOPTH

Kind

Abbildung 80: Tennismerkmal SBW1 bei den Kindern 


\section{Bewegungslernen}

\section{Merkmal SBW2}

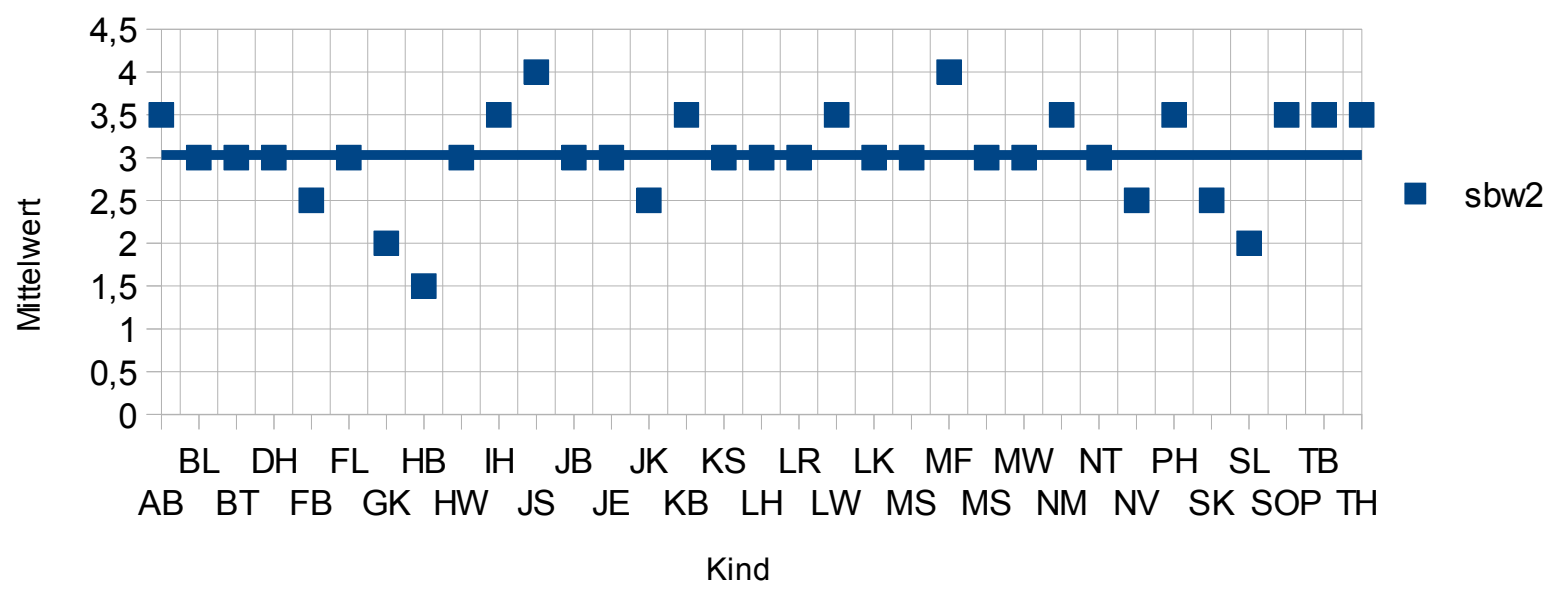

Abbildung 81: Tennismerkmal SBW2 bei den Kindern

\section{Bewegungslernen}

\section{Merkmal SBW3}

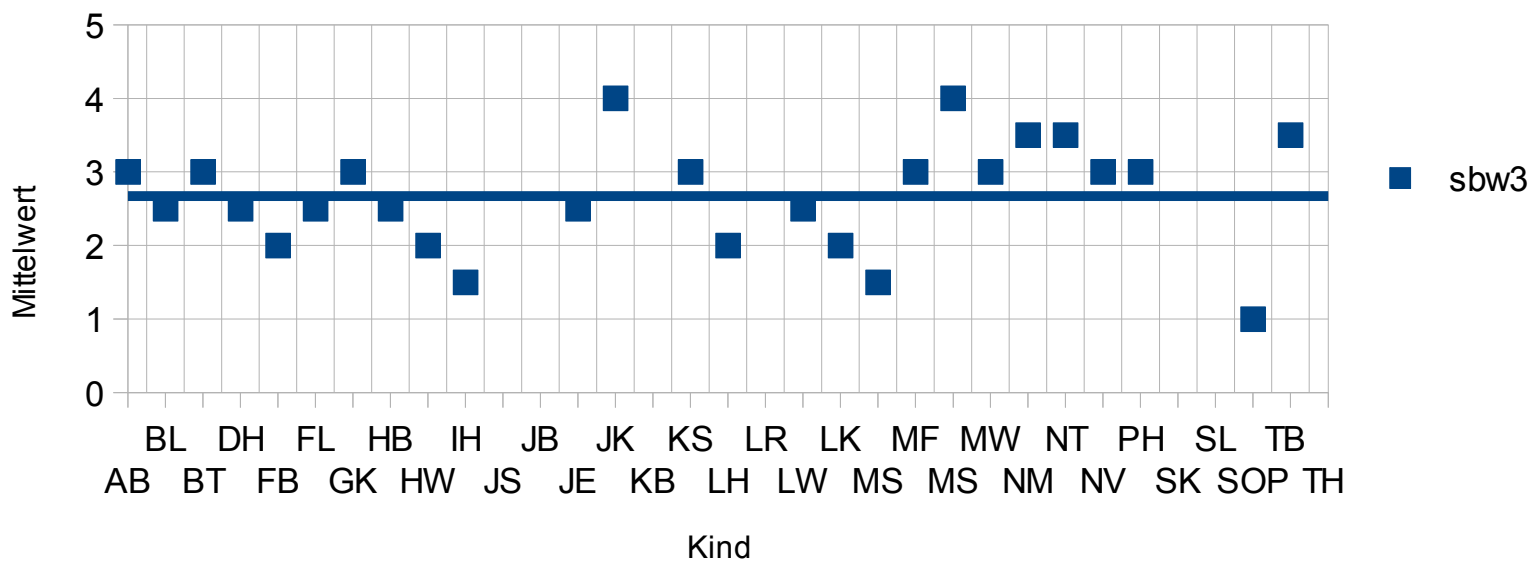

Abbildung 82: Tennismerkmal SBW3 bei den Kindern 


\section{Bewegungslernen}

Merkmal AB1

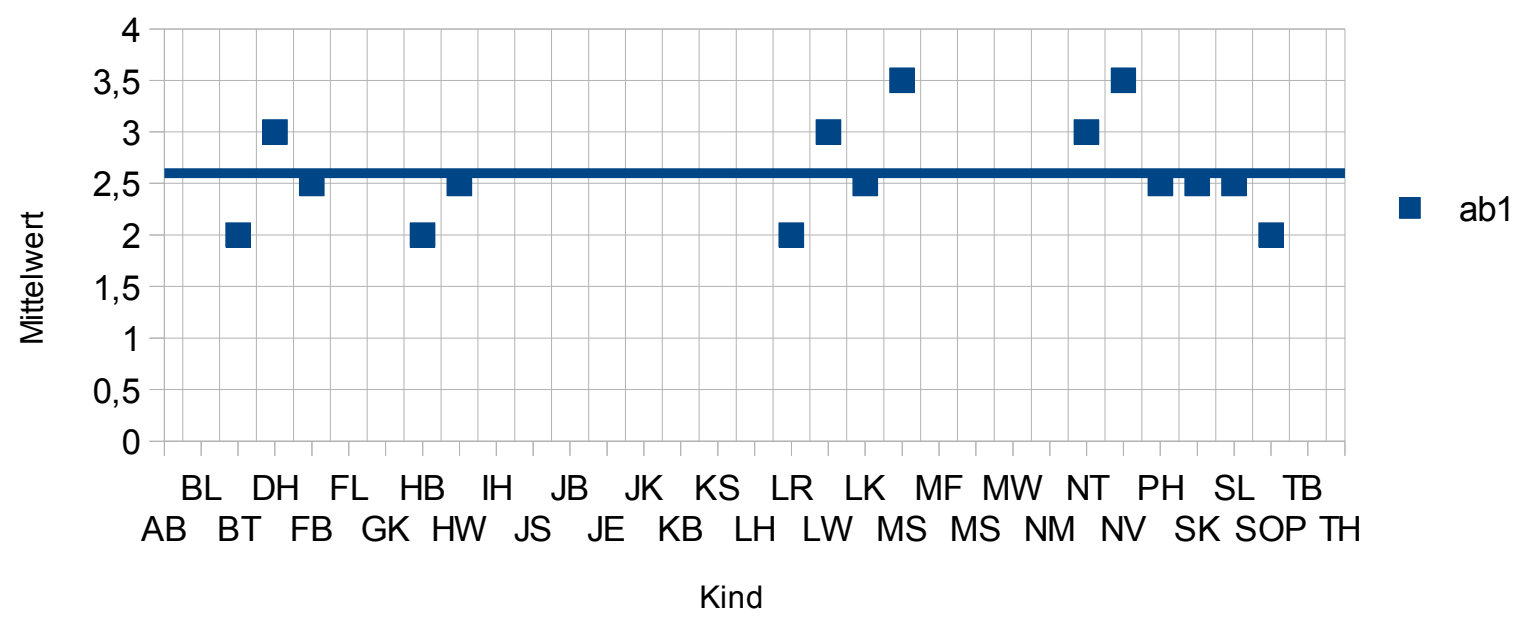

Abbildung 83: Tennismerkmal AB1 bei den Kindern

\section{Bewegungslernen}

Merkmal AB2

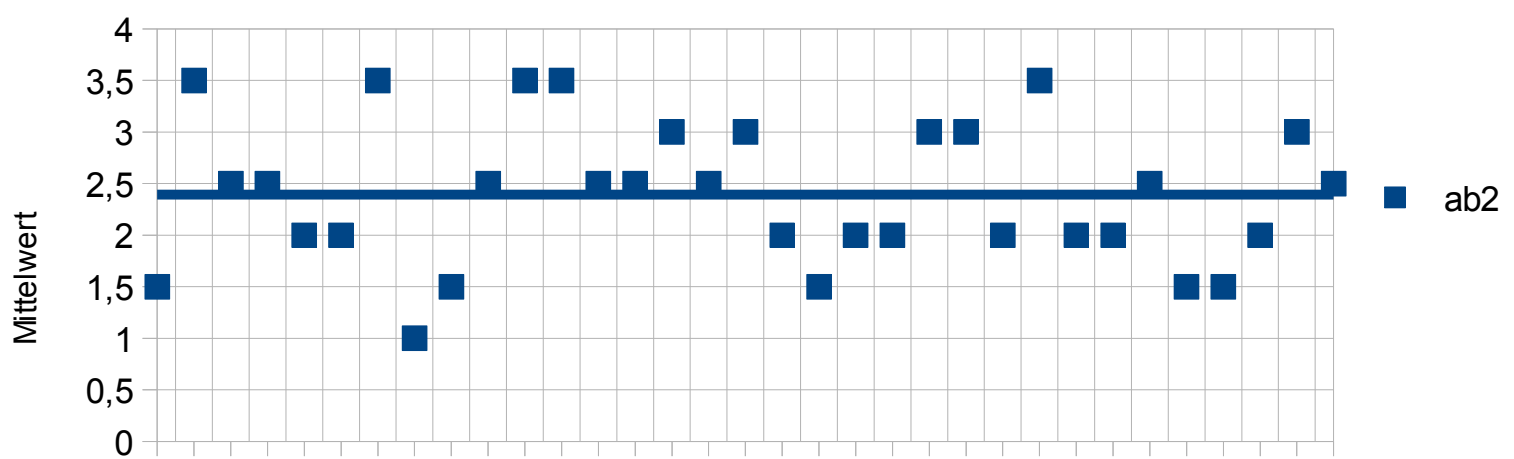

BL $\quad D H \quad F L \quad H B \quad I H \quad J B \quad J K \quad K S \quad L R \quad L K \quad M F \quad M W \quad N T$ PH SL TB AB BT FB GK HW JS JE KB LH LW MS MS NM NV SK SOP TH Kind 


\section{Bewegungslernen}

\section{Merkmal AB3}

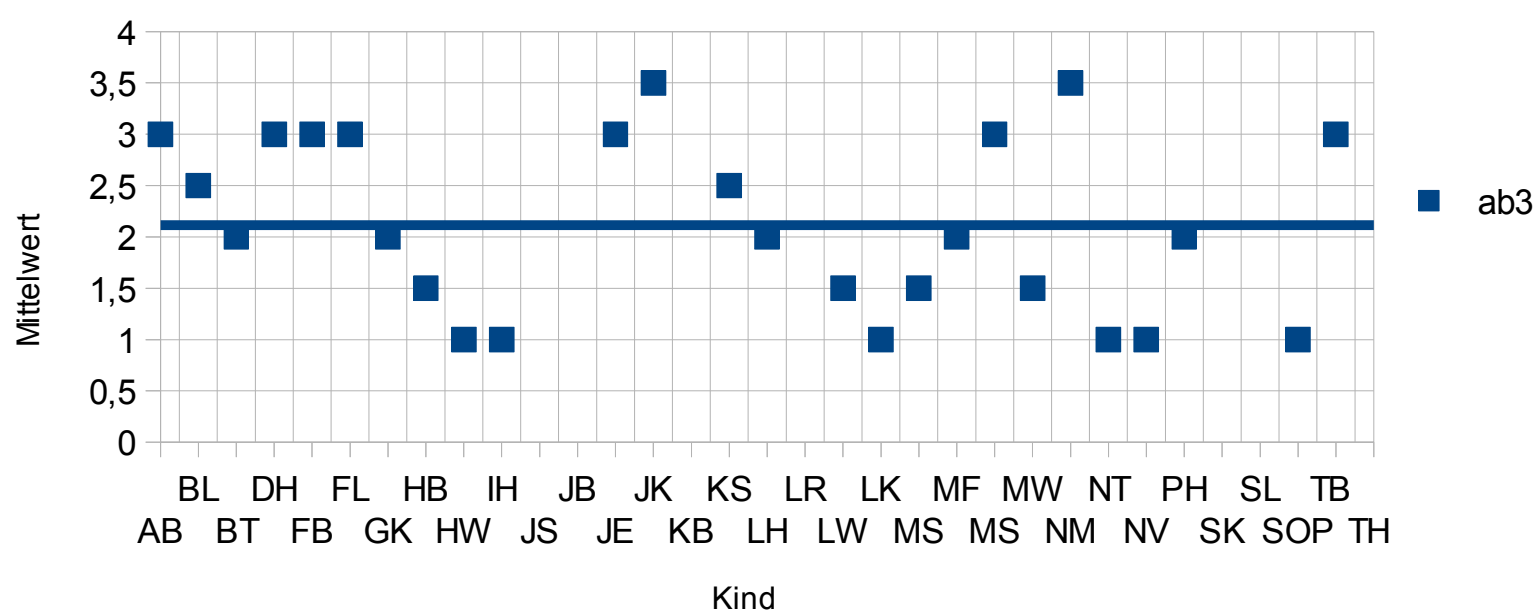

Abbildung 85: Tennismerkmal AB3 bei den Kindern

\section{Bewegungslernen}

Merkmal BK1

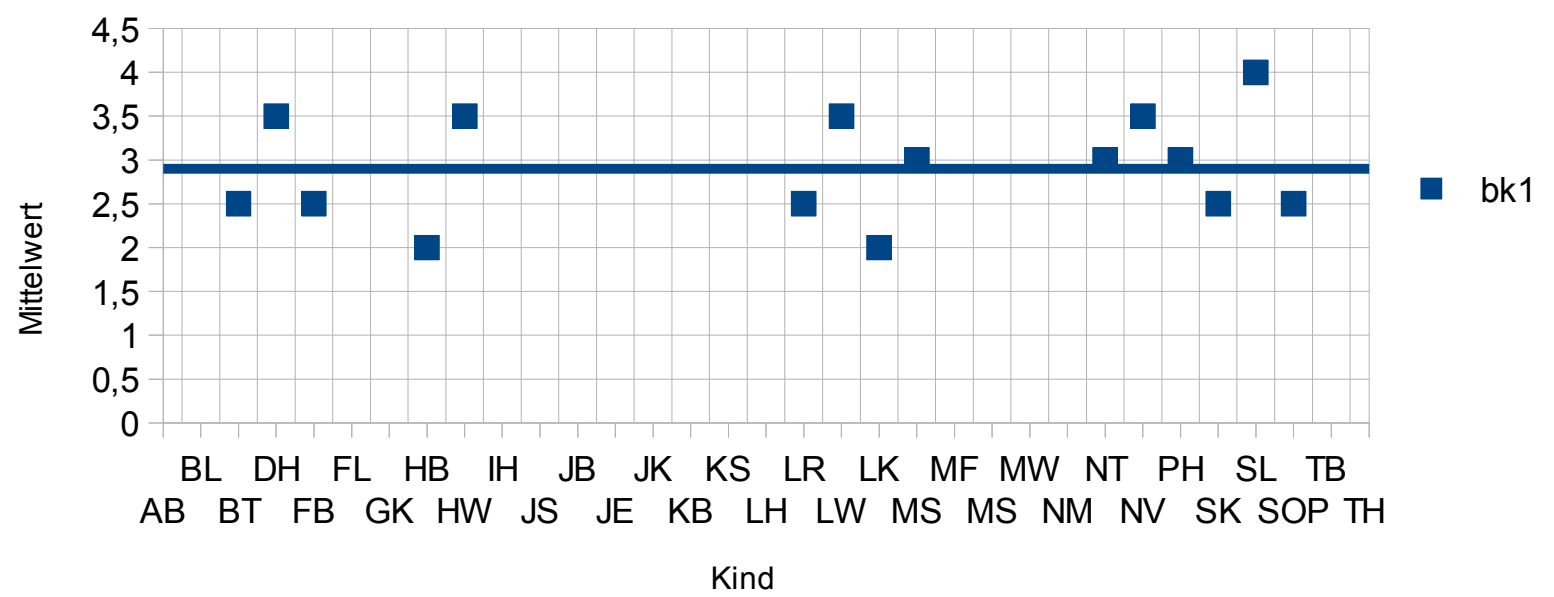

Abbildung 86: Tennismerkmal BK1 bei den Kindern 


\section{Bewegungslernen}

\section{Merkmal BK2}

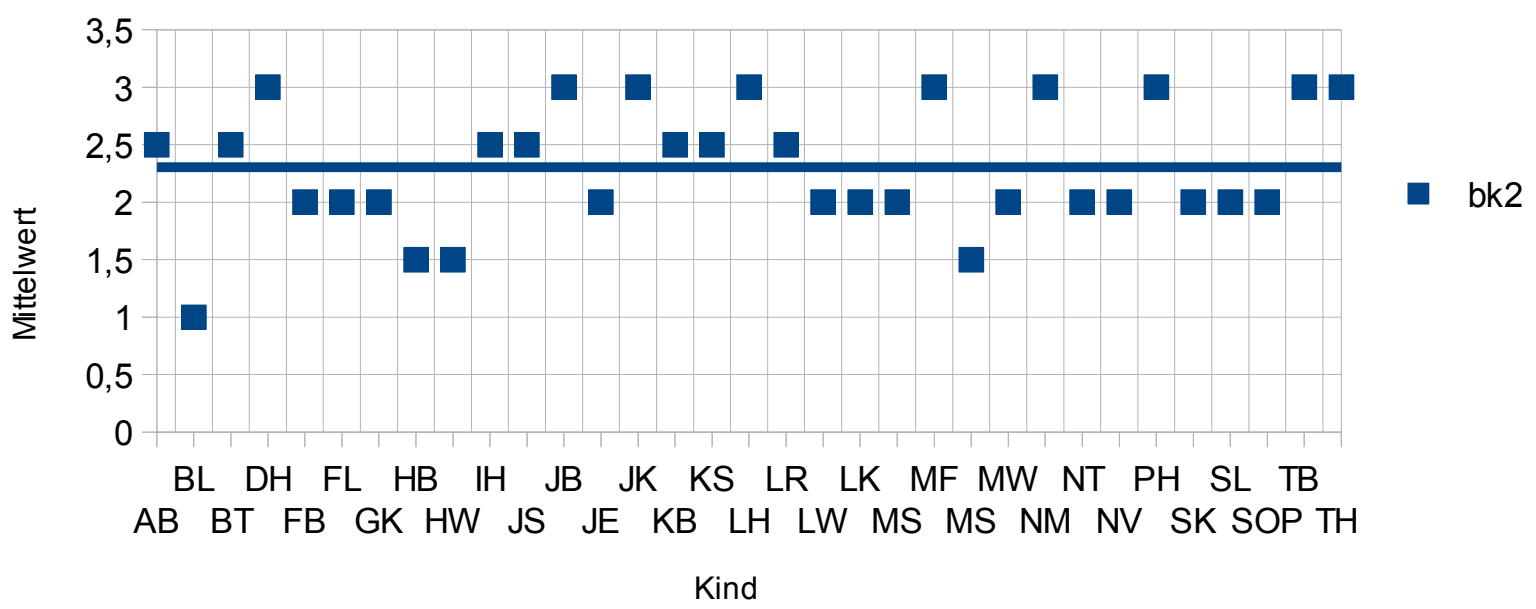

Abbildung 87: Tennismerkmal BK2 bei den Kindern

\section{Bewegungslernen}

Merkmal BK3

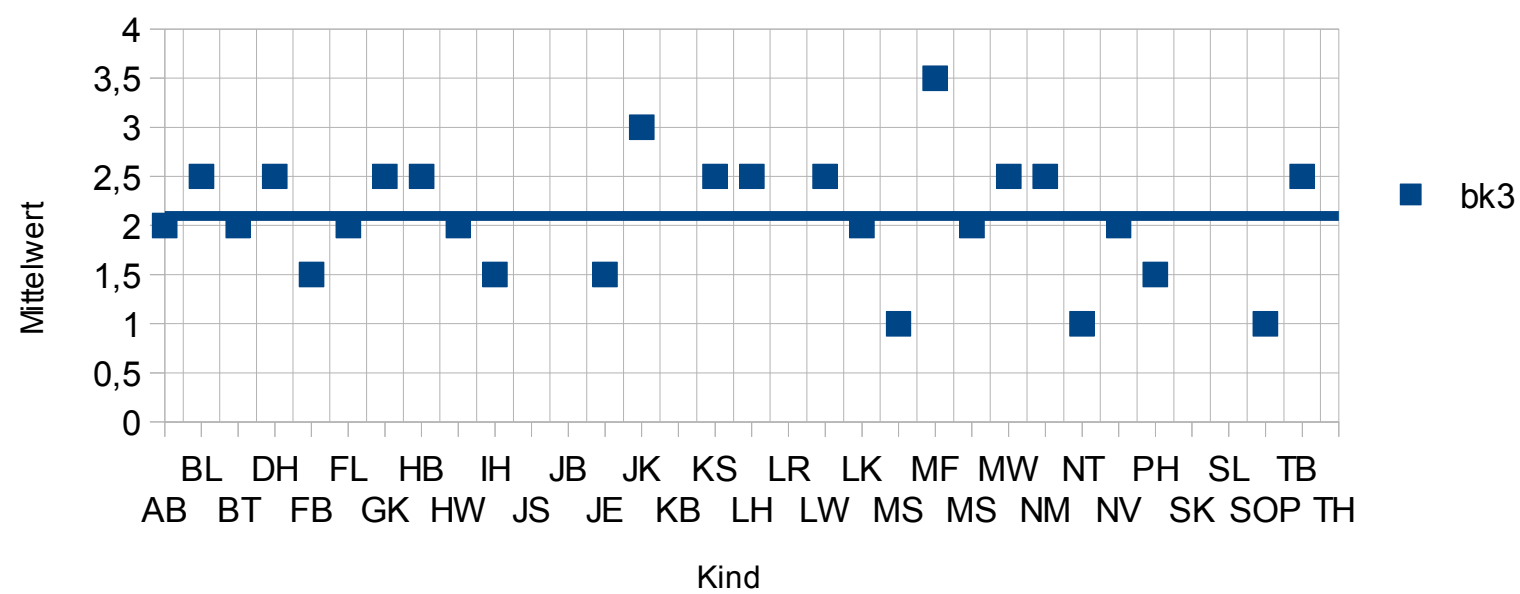

Abbildung 88: Tennismerkmal BK3 bei den Kindern 


\subsection{Rangfolge der Kinder/Entwicklungsstabilität}

Es werden Mittelwerte über alle Tennismerkmale gebildet, so dass eine Rangfolge der Kinder zu den drei Messzeitpunkten ermittelt werden kann (siehe Tabellen 58 bis 60).

\begin{tabular}{|l|c|c|c|c|c|c|c|c|c|c|c|c|c|}
\hline NAME & sp1 & aba1 & abb1 & sbg1 & sbt1 & sbf1 & sbk1 & sbgk1 & sbva1 & sbw1 & ab1 & bk1 & Mittel \\
\hline HB & 1,5 & 1,5 & 2 & 1,5 & 2 & 2,5 & 2,5 & 2,5 & 1,5 & 1 & 2 & 2 & 1,88 \\
\hline LR & 1,5 & 2 & 2 & 2,5 & 2 & 3 & 3 & 2,5 & 1,5 & 1,5 & 2 & 2,5 & 2,17 \\
\hline SOP & 2 & 2 & 2 & 2 & 2,5 & 2,5 & 2,5 & 2,5 & 2 & 3 & 2 & 2,5 & 2,29 \\
\hline LK & 2 & 2 & 2 & 2 & 2 & 3 & 2,5 & 2,5 & 3 & 3,5 & 2,5 & 2 & 2,42 \\
\hline NT & 2,5 & 2 & 2,5 & 2 & 2 & 2 & 3 & 2,5 & 2 & 4 & 3 & 3 & 2,54 \\
\hline BT & 2,5 & 3 & 3 & 2 & 3 & 2 & 3 & 2 & 2,5 & 3,5 & 2 & 2,5 & 2,58 \\
\hline MAS & 2 & 2 & 2,5 & 2 & 3 & 2 & 3 & 2 & 2 & 4 & 3,5 & 3 & 2,58 \\
\hline SK & 2 & 2 & 3 & 2 & 3 & 3 & 3 & 2,5 & 3 & 3 & 2,5 & 2,5 & 2,63 \\
\hline DH & 2,5 & 2,5 & 3 & 3 & 3 & 2,5 & 2 & 2,5 & 2 & 3 & 3 & 3,5 & 2,71 \\
\hline FB & 2,5 & 2,5 & 2,5 & 2 & 3 & 3,5 & 3 & 3 & 2 & 3,5 & 2,5 & 2,5 & 2,71 \\
\hline HW & 2,5 & 3 & 2,5 & 2 & 2,5 & 3,5 & 2,5 & 3 & 2 & 3,5 & 2,5 & 3,5 & 2,75 \\
\hline LW & 2,5 & 2,5 & 2,5 & 2 & 3,5 & 3 & 3 & 2,5 & 2 & 3 & 3 & 3,5 & 2,75 \\
\hline PH & 3 & 3,5 & 3 & 2,5 & 3,5 & 2 & 3 & 3 & 2 & 3 & 2,5 & 3 & 2,83 \\
\hline SL & 3 & 2,5 & 3 & 2 & 3,5 & 3 & 2,5 & 3 & 2 & 3 & 2,5 & 4 & 2,83 \\
\hline NV & 2,5 & 2 & 2,5 & 3 & 3 & 3 & 3 & 3 & 2 & 4 & 3,5 & 3,5 & 2,92 \\
\hline
\end{tabular}

Tabelle 58: Rangfolge der Kinder zu allen Tennismerkmalen zum Messzeitpunkt 1

Dabei entsprechen geringe Werte hohen Bewegungsfertigkeiten. 


\begin{tabular}{|c|c|c|c|c|c|c|c|c|c|c|c|c|c|}
\hline NAME & $\mathrm{sp} 2$ & aba2 & abb2 & sbg2 & sbt2 & sbf2 & sbk2 & sbgk2 & sbva2 & sbw2 & ab2 & bk2 & Mittel \\
\hline $\mathrm{HB}$ & 1 & 1 & 1 & 1 & 1,5 & 3 & 2,5 & 2,5 & 2 & 1,5 & 1 & 1,5 & 1,63 \\
\hline $\mathrm{HW}$ & 2 & 2 & 2 & 1 & 2 & 2 & 2 & 2 & 1 & 3 & 1,5 & 1,5 & 1,83 \\
\hline SL & 2 & 2 & 2 & 1,5 & 2 & 3 & 2,5 & 2,5 & 2 & 2 & 1,5 & 2 & 2,08 \\
\hline NV & 2 & 2 & 2 & 1,5 & 2,5 & 2,5 & 2,5 & 2 & 2 & 2,5 & 2 & 2 & 2,13 \\
\hline LK & 1,5 & 2 & 1,5 & 1,5 & 2 & 3 & 2,5 & 2,5 & 2,5 & 3 & 2 & 2 & 2,17 \\
\hline MS & 1,5 & 2 & 2 & 1,5 & 2 & 3 & 2,5 & 2,5 & 2 & 3 & 3 & 1,5 & 2,21 \\
\hline FB & 2,5 & 2,5 & 2 & 1,5 & 2 & 3 & 3 & 2,5 & 2 & 2,5 & 2 & 2 & 2,29 \\
\hline $\mathrm{FL}$ & 2,5 & 2 & 2,5 & 2 & 2 & 2 & 3 & 2,5 & 2 & 3 & 2 & 2 & 2,29 \\
\hline MAS & 2,5 & 2 & 2 & 2 & 2 & 3,5 & 2,5 & 2,5 & 1,5 & 3 & 2 & 2 & 2,29 \\
\hline SK & 2 & 2,5 & 2,5 & 1,5 & 2,5 & 3 & 3 & 2,5 & 2 & 2,5 & 1,5 & 2 & 2,29 \\
\hline SOP & 1,5 & 1,5 & 2,5 & 2 & 2,5 & 3 & 2,5 & 2,5 & 2 & 3,5 & 2 & 2 & 2,29 \\
\hline JE & 2 & 2 & 2 & 1,5 & 2 & 3,5 & 3 & 3 & 2 & 3 & 2,5 & 2 & 2,38 \\
\hline LW & 2 & 1,5 & 2 & 2 & 2 & 3 & 3 & 3 & 3 & 3,5 & 1,5 & 2 & 2,38 \\
\hline MW & 2,5 & 2 & 2 & 2 & 2 & 3,5 & 3,5 & 3 & 2 & 3 & 2 & 2 & 2,46 \\
\hline NT & 2,5 & 2 & 2,5 & 2 & 3 & 3,5 & 3 & 2,5 & 2 & 3 & 2 & 2 & 2,50 \\
\hline $\mathrm{IH}$ & 2,5 & 2 & 2,5 & 2 & 3 & 2,5 & 3 & 2,5 & 2 & 3,5 & 2,5 & 2,5 & 2,54 \\
\hline BT & 2,5 & 3 & 3 & 2 & 3 & 2,5 & 3 & 2,5 & 1,5 & 3 & 2,5 & 2,5 & 2,58 \\
\hline LR & 3 & 2 & 3 & 2 & 3,5 & 2,5 & 3 & 2,5 & 2 & 3 & 2 & 2,5 & 2,58 \\
\hline$B L$ & 2 & 2,5 & 2,5 & 2 & 2,5 & 3,5 & 3,5 & 3,5 & 2 & 3 & 3,5 & 1 & 2,63 \\
\hline GK & 2,5 & 2,5 & 3 & 2 & 3,5 & 3,5 & 2,5 & 2,5 & 2 & 2 & 3,5 & 2 & 2,63 \\
\hline JK & 2,5 & 2,5 & 2,5 & 2 & 3,5 & 3 & 3 & 2,5 & 2 & 2,5 & 2,5 & 3 & 2,63 \\
\hline AB & 3 & 3 & 2,5 & 1,5 & 3 & 3,5 & 3,5 & 2,5 & 2 & 3,5 & 1,5 & 2,5 & 2,67 \\
\hline KB & 2,5 & 2,5 & 2 & 2 & 3 & 3 & 3 & 3 & 2 & 3,5 & 3 & 2,5 & 2,67 \\
\hline JS & 2,5 & 3,5 & 2 & 2,5 & 2 & 3,5 & 2 & 2,5 & 2 & 4 & 3,5 & 2,5 & 2,71 \\
\hline KS & 3 & 2,5 & 2,5 & 2 & 3 & 3,5 & 3 & 3 & 2 & 3 & 2,5 & 2,5 & 2,71 \\
\hline LH & 2,5 & 2 & 2,5 & 2 & 3,5 & 3,5 & 3 & 2,5 & 2,5 & 3 & 3 & 3 & 2,75 \\
\hline $\mathrm{TH}$ & 3,5 & 2,5 & 2,5 & 2 & 2,5 & 3 & 3,5 & 3 & 2 & 3,5 & 2,5 & 3 & 2,79 \\
\hline $\mathrm{DH}$ & 3 & 3 & 3 & 2 & 3,5 & 3,5 & 3 & 3 & 2 & 3 & 2,5 & 3 & 2,88 \\
\hline $\mathrm{PH}$ & 3 & 3 & 2,5 & 3 & 2,5 & 3,5 & 3 & 3 & 2 & 3,5 & 2,5 & 3 & 2,88 \\
\hline MF & 3,5 & 2,5 & 2,5 & 2 & 3,5 & 3,5 & 3 & 3 & 2 & 4 & 3 & 3 & 2,96 \\
\hline TB & 3 & 3 & 3 & 2,5 & 3,5 & 3,5 & 3 & 2,5 & 2 & 3,5 & 3 & 3 & 2,96 \\
\hline$J B$ & 3 & 3,5 & 3 & 2,5 & 3 & 3,5 & 3,5 & 3,5 & 2 & 3 & 3,5 & 3 & 3,08 \\
\hline NM & 4 & 3,5 & 3 & 2 & 4 & 3,5 & 3,5 & 3,5 & 2 & 3,5 & 3,5 & 3 & 3,25 \\
\hline
\end{tabular}

Tabelle 59: Rangfolge der Kinder zu allen Tennismerkmalen zum Messzeitpunkt 2 


\begin{tabular}{|l|c|c|c|c|c|c|c|c|c|c|c|c|c|}
\hline NAME & sp3 & aba3 & abb3 & sbg3 & sbt3 & sbf3 & sbk3 & sbgk3 & sbva3 & sbw3 & ab3 & bk3 & Mittel \\
\hline SOP & 1 & 1 & 1 & 1,5 & 1 & 1,5 & 1 & 1,5 & 1 & 1 & 1 & 1 & 1,13 \\
\hline MAS & 1 & 1 & 1 & 1 & 1 & 2 & 2 & 1,5 & 1 & 1,5 & 1,5 & 1 & 1,29 \\
\hline IH & 2 & 1 & 1 & 1 & 2 & 2 & 1 & 2 & 2 & 1,5 & 1 & 1,5 & 1,50 \\
\hline FB & 1 & 1,5 & 1,5 & 1,5 & 2 & 2,5 & 1,5 & 2 & 1,5 & 2 & 3 & 1,5 & 1,79 \\
\hline JE & 1 & 1 & 1 & 1 & 2 & 2 & 3 & 2 & 1,5 & 2,5 & 3 & 1,5 & 1,79 \\
\hline LK & 1,5 & 1,5 & 2 & 1 & 2 & 3 & 2 & 2 & 1,5 & 2 & 1 & 2 & 1,79 \\
\hline HW & 2 & 1,5 & 2,5 & 1,5 & 2,5 & 2,5 & 1,5 & 2 & 1,5 & 2 & 1 & 2 & 1,88 \\
\hline BT & 2 & 2 & 2 & 1,5 & 2 & 1,5 & 2 & 1,5 & 2 & 3 & 2 & 2 & 1,96 \\
\hline LW & 2,5 & 1,5 & 2 & 1,5 & 2 & 2,5 & 2 & 2 & 2,5 & 2,5 & 1,5 & 2,5 & 2,08 \\
\hline NV & 2 & 1,5 & 2,5 & 1,5 & 2,5 & 3 & 2 & 2 & 2 & 3 & 1 & 2 & 2,08 \\
\hline PH & 1,5 & 2 & 2 & 2 & 2 & 3,5 & 2 & 2,5 & 1,5 & 3 & 2 & 1,5 & 2,13 \\
\hline HB & 2 & 1,5 & 2 & 1 & 2,5 & 3,5 & 2,5 & 2,5 & 2 & 2,5 & 1,5 & 2,5 & 2,17 \\
\hline NT & 2,5 & 1,5 & 2 & 2,5 & 1,5 & 3,5 & 3 & 3 & 2 & 3,5 & 1 & 1 & 2,25 \\
\hline AB & 2,5 & 2 & 2,5 & 1,5 & 2,5 & 2,5 & 2 & 2 & 2 & 3 & 3 & 2 & 2,29 \\
\hline BL & 2,5 & 1,5 & 2,5 & 2,5 & 2,5 & 2,5 & 2,5 & 2 & 2 & 2,5 & 2,5 & 2,5 & 2,33 \\
\hline LH & 3 & 2 & 2,5 & 2 & 3 & 2 & 2,5 & 2,5 & 2 & 2 & 2 & 2,5 & 2,33 \\
\hline DH & 2 & 2 & 2 & 2,5 & 2 & 3,5 & 2 & 2,5 & 2 & 2,5 & 3 & 2,5 & 2,38 \\
\hline MS & 2,5 & 2 & 3 & 2 & 3 & 2 & 2 & 2 & 2 & 4 & 3 & 2 & 2,46 \\
\hline MW & 2,5 & 2,5 & 2,5 & 2 & 3 & 3,5 & 2 & 2,5 & 2 & 3 & 1,5 & 2,5 & 2,46 \\
\hline FL & 2,5 & 3,5 & 2,5 & 1,5 & 2,5 & 3 & 2,5 & 2,5 & 2 & 2,5 & 3 & 2 & 2,50 \\
\hline GK & 2 & 2 & 3 & 2 & 2,5 & 3,5 & 3 & 3 & 2 & 3 & 2 & 2,5 & 2,54 \\
\hline MF & 3 & 2 & 2 & 3 & 2 & 3 & 2,5 & 2,5 & 2 & 3 & 2 & 3,5 & 2,54 \\
\hline KS & 3 & 2,5 & 3 & 2 & 3 & 3 & 2 & 2,5 & 2,5 & 3 & 2,5 & 2,5 & 2,63 \\
\hline TB & 3,5 & 2,5 & 2,5 & 3,5 & 3 & 3 & 2,5 & 2,5 & 1,5 & 3,5 & 3 & 2,5 & 2,79 \\
\hline NM & 3,5 & 3,5 & 3 & 2 & 4 & 2,5 & 2 & 2,5 & 1,5 & 3,5 & 3,5 & 2,5 & 2,83 \\
\hline JK & 4 & 3,5 & 2,5 & 3,5 & 3 & 1,5 & 2,5 & 1,5 & 2 & 4 & 3,5 & 3 & 2,88 \\
\hline
\end{tabular}

Tabelle 60: Rangfolge der Kinder zu allen Tennismerkmalen zum Messzeitpunkt 3 


\subsection{Tabellenverzeichnis}

Tabelle 1: Ergebnisse des Experiments von Brooks (1968), Reaktionszeiten in Sekunden für die

Klassifikation

Tabelle 2: Der Ablauf der Studie (Legende: Basisbedingung 0, d.h. ohne Flächenabdeckung, $25=25 \%$

Flächenabdeckung, 33z=33\% zusammenhängende Flächenabdeckung, 33g=33\% getrennte

Flächenabdeckung, 50z/50g= 50\% zusammenhängende/getrennte Flächenabdeckung)

Tabelle 3: Alter in der Versuchsgruppe (Anmerkung: Alter0=Alter in der Wahrnehmungsbedingung „0“)...93

Tabelle 4: Interraterreliabilität zu verschiedenen Messzeitpunkten

Tabelle 5: Mittelwerte (Standardabweichungen) und Fallzahlen für das Bewegungslernen an drei

Messzeitpunkten

Tabelle 6: Mittelwerte (links) und Standardabweichung (rechts) der Tennismerkmale an drei

Messzeitpunkten, MZP1 (N=9), MZP2 ( $=24)$, MZP3 ( $N=21)$. 101

Tabelle 7: Ergebnisse des Bewegungslernens über alle Kinder der Versuchsgruppe (rot: signifikant, blau: Trend). 102

Tabelle 8: Korrelationen von Vorstellungs- und Wahrnehmungsleistungen mit dem Bewegungslernen zum Messzeitpunkt 2. 105

Tabelle 9: Korrelationen von Vorstellungs- und Wahrnehmungsleistungen mit dem Bewegungslernen zum Messzeitpunkt 3 105

Tabelle 10: Zusammenhänge zwischen den Subtests des Experiments und dem Tennismerkmal SP2 ...106 Tabelle 11: Zusammenhänge zwischen den Subtests des Experiments und dem Tennismerkmal ABA2..106 Tabelle 12: Zusammenhänge zwischen den Subtests des Experiments und dem Tennismerkmal ABB2..106 Tabelle 13: Zusammenhänge zwischen den Subtests des Experiments und dem Tennismerkmal SBG2. 107 Tabelle 14: Zusammenhänge zwischen den Subtests des Experiments und dem Tennismerkmal SBT2..107 Tabelle 15: Zusammenhänge zwischen den Subtests des Experiments und dem Tennismerkmal SBF2..107 Tabelle 16: Zusammenhänge zwischen den Subtests des Experiments und dem Tennismerkmal SBK2..107 Tabelle 17: Zusammenhänge zwischen den Subtests des Experiments und dem Tennismerkmal SBGK2

Tabelle 18: Zusammenhänge zwischen den Subtests des Experiments und dem Tennismerkmal SBVA2

Tabelle 19: Zusammenhänge zwischen den Subtests des Experiments und dem Tennismerkmal SBW2.108 Tabelle 20: Zusammenhänge zwischen den Subtests des Experiments und dem Tennismerkmal AB2 ...108 Tabelle 21: Zusammenhänge zwischen den Subtests des Experiments und dem Tennismerkmal BK2...109 Tabelle 22: Zusammenhänge zwischen den Subtests des Experiments und dem Tennismerkmal SP3....109 Tabelle 23: Zusammenhänge zwischen den Subtests des Experiments und dem Tennismerkmal ABA3. .110 Tabelle 24: Zusammenhänge zwischen den Subtests des Experiments und dem Tennismerkmal ABB3. .110 Tabelle 25: Zusammenhänge zwischen den Subtests des Experiments und dem Tennismerkmal SBG3. 110 Tabelle 26: Zusammenhänge zwischen den Subtests des Experiments und dem Tennismerkmal SBT3. .110 Tabelle 27: Zusammenhänge zwischen den Subtests des Experiments und dem Tennismerkmal SBF3. .111 Tabelle 28: Zusammenhänge zwischen den Subtests des Experiments und dem Tennismerkmal SBGK3

Tabelle 29: Zusammenhänge zwischen den Subtests des Experiments und dem Tennismerkmal SBVA3 111 
Tabelle 30: Zusammenhänge zwischen den Subtests des Experiments und dem Tennismerkmal ABK3. .111

Tabelle 31: Zusammenhänge zwischen den Subtests des Experiments und dem Tennismerkmal SBW3. 112

Tabelle 32: Zusammenhänge zwischen den Subtests des Experiments und dem Tennismerkmal AB3....112

Tabelle 33: Zusammenhänge zwischen den Subtests des Experiments und dem Tennismerkmal BK3....112

Tabelle 34: durchschnittliche Ausprägung der Tennistechnik und Rangfolge der Mittelwerte zu den drei

Messzeitpunkten (durch das Expertenrating ermittelt) (Anmerkung: hier sind die genauen Mittelwerte eingetragen, während bei den T-Tests oben ein Fallausschluss die Mittelwerte beeinflusst).

Tabelle 35: Alter der Gesamtgruppe in der deskriptiven Statistik zu den einzelnen Teilversuchs

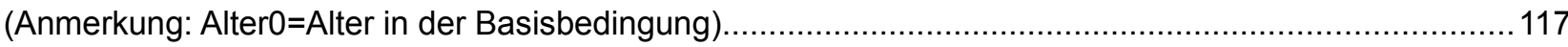

Tabelle 36: Alter in der Versuchsgruppe (Anmerkung: Alter0=Alter in der Basisbedingung) ....................118

Tabelle 37: Alter in der Kontrollgruppe (Anmerkung: Alter0=Alter in der Basisbedingung) .......................118

Tabelle 38: deskriptive Statistik für die Faktoren Gruppe, Geschlecht und Versuchsbedingung hinsichtlich

der Wahrnehmungs- und Vorstellungs-leistung

Tabelle 39: Gesamtmittel der Wahrnehmungs- und Vorstellungsleistung ........................................... 121

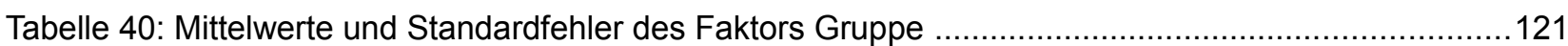

Tabelle 41: Mittelwerte und Standardfehler der Faktoren Gruppe und Versuchsbedingung....................122

Tabelle 42: Mittelwerte und Standardfehler des Faktors Geschlecht..................................................126

Tabelle 43: Mittelwerte und Standardfehler der Faktoren Gruppe und Geschlecht...............................127

Tabelle 44: Mittelwerte und Standardfehler der Faktoren Geschlecht und Versuchsbedingung...............128

Tabelle 45: Mittelwerte und Standardfehler des Messwiederholungsfaktors Versuchsbedingung............133

Tabelle 46: Ergebnisse der deskriptiven Statistik (Anmerkung: jüngere Kinder (1), ältere Kinder (2))......136

Tabelle 47: Ergebnisse des Levene-Tests (Anmerkung df Zähler=1 df Nenner=5, N=7).....................137

Tabelle 48: Ergebnisse der Signifikanztests hinsichtlich des Erfahrungsfaktors zu den Wahrnehmungs- und

Vorstellungsleistungen

Tabelle 49: Mittelwerte und Standardabweichungen des Faktors Trainingserfahrung für Veränderungen im

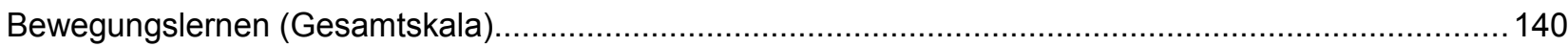

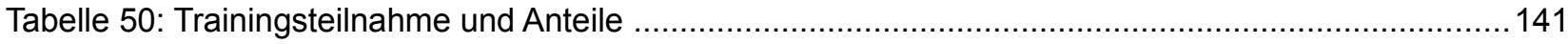

Tabelle 51: bewegungsnahe und bewegungsferne Aktivtiäten bei den Kindern des Projekts „Jüngsten-

Tennis“ (in Minuten), Verhältnis von bewegungsnahen zu bewegungsfernen Aktivitäten .........................142

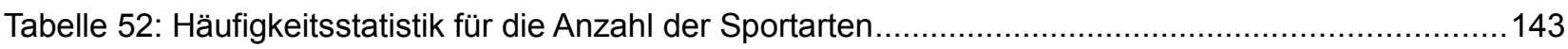

Tabelle 53: Ergebnisse des Kasten-Bumerang-Laufs. (Anmerkung: Die Punktzahl ist altersnormiert).

(Legende: F= Fehler während der Durchführung, Punktzahl 4= weit überdurchschnittlich, 3=

überdurchschnittlich, 2= durchschnittlich, $1=$ unterdurchschnittlich, $0=$ weit unterdurchschnittlich)..........144

Tabelle 54: Punktzahl und Anteile beim Kasten-Bumerang-Test......................................................... 145

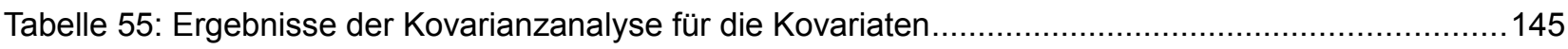

Tabelle 56: Mittelwerte (Standardabweichungen) des Faktors Geschlecht zum Bewegungslernen

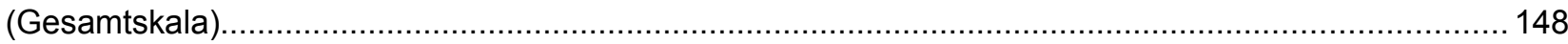

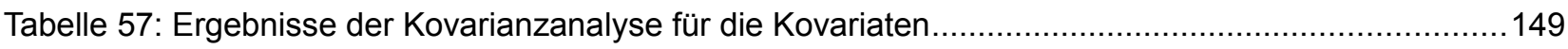

Tabelle 58: Rangfolge der Kinder zu allen Tennismerkmalen zum Messzeitpunkt 1 ..............................192

Tabelle 59: Rangfolge der Kinder zu allen Tennismerkmalen zum Messzeitpunkt 2 ............................193

Tabelle 60: Rangfolge der Kinder zu allen Tennismerkmalen zum Messzeitpunkt 3...............................194 


\subsection{Abbildungsverzeichnis}

Abbildung 1: Vorstellungsmodell von Farah (1984, S. 250).

Abbildung 2: Modell von Finke (1986) zum Einfluss von Vorstellungen auf die Wahrnehmung (zit. nach

Kebeck, 1997, S. 197).

Abbildung 3: Das Prototypen-Modell der Wahrnehmung ähnelt dem des Vorstellungsprozesses, Kosslyn

(1994; S. 69 und 383) (vereinfacht und zusammengefasst)......

Abbildung 4: Ergebnisse des Experiments urspr. nach Ishai und Sagi (1997a, S. 485); die

vorstellungsinduzierte Erleichterung der Wahrnehmungsschwelle

Abbildung 5: Ergebnisse des Experiments urspr. nach Ishai und Sagi (1997b, S. 737) .........................61

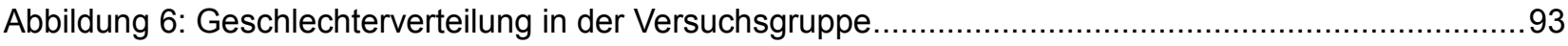

Abbildung 7: Skizze Untersuchungsmaterial und -ablauf (die Trapeze kennzeichnen die Zeitlinie)............95

Abbildung 8: Bewegungslernen über drei Messzeitpunkte in der VG............................................ 100

Abbildung 9: Bewegungslernen anhand der verschiedenen Tennismerkmale zu den drei Messzeitpunkten

Abbildung 10: Entwicklung der Tennistechnik über 3 Messzeitpunkte bei Kind SP...............................113

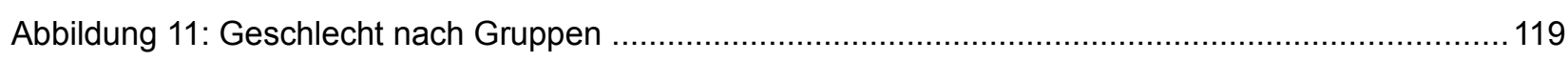

Abbildung 12: Ergebnisse der Varianzanalyse mit Messwiederholungen für die Wahrnehmungs- und

Vorstellungsleistung nach Gruppen

Abbildung 13: Interaktion von Gruppe und Versuchsbedingung hinsichtlich der Wahrnehmungs- und

Vorstellungsleistung.

Abbildung 14: Ergebnisse der Varianzanalyse zum Faktor Geschlecht über alle Versuchsbedingungen..127

Abbildung 15: Ergebnisse der Varianzanalyse zum den Faktoren Gruppe und Geschlecht 128

Abbildung 16: Ergebnisse der Varianzanalyse zur Wahrnehmungs- und Vorstellung bezüglich der

Faktoren Versuchsbedingung und Geschlecht

Abbildung 17: Ergebnisse der Varianzanalyse mit Messwiederholungen für die Wahrnehmungs- und

Vorstellungsleistung nach Versuchsbedingungen.

Abbildung 18: Ergebnis der Kovarianzanalyse zum Faktor Trainingserfahrung und dessen Einfluss auf das

Bewegungslernen (Gesamtskala) (Jüngere und ältere Kinder wurden in den zwei verschiedenen

Trainingsgruppen jeweils unterschiedlich beschult.).

Abbildung 19: Ergebnis der Kovarianzanalyse zum Faktor Geschlecht und dessen Einfluss auf das

Bewegungslernen (Gesamtskala) 148

Abbildung 20: Entwicklung der Tennistechnik über 3 Messzeitpunkte bei Kind AB..............................159

Abbildung 21: Entwicklung der Tennistechnik über 3 Messzeitpunkte bei Kind BL..................................159

Abbildung 22: Entwicklung der Tennistechnik über 3 Messzeitpunkte bei Kind BT ................................160

Abbildung 23: Entwicklung der Tennistechnik über 3 Messzeitpunkte bei Kind DH................................160

Abbildung 24: Entwicklung der Tennistechnik über 3 Messzeitpunkte bei Kind FB................................161

Abbildung 25: Entwicklung der Tennistechnik über 3 Messzeitpunkte bei Kind FL...............................161

Abbildung 26: Entwicklung der Tennistechnik über 3 Messzeitpunkte bei Kind GK.................................162

Abbildung 27: Entwicklung der Tennistechnik über 3 Messzeitpunkte bei Kind HB..............................162

Abbildung 28: Entwicklung der Tennistechnik über 3 Messzeitpunkte bei Kind HW.............................163

Abbildung 29: Entwicklung der Tennistechnik über 3 Messzeitpunkte bei Kind IH.................................163 
Abbildung 30: Entwicklung der Tennistechnik über 3 Messzeitpunkte bei Kind KB.............................164

Abbildung 31: Entwicklung der Tennistechnik über 3 Messzeitpunkte bei Kind JK .................................164

Abbildung 32: Entwicklung der Tennistechnik über 3 Messzeitpunkte bei Kind JE...............................164

Abbildung 33: Entwicklung der Tennistechnik über 3 Messzeitpunkte bei Kind JB...................................164

Abbildung 34: Entwicklung der Tennistechnik über 3 Messzeitpunkte bei Kind JS................................164

Abbildung 35: Entwicklung der Tennistechnik über 3 Messzeitpunkte bei Kind KS...............................164

Abbildung 36: Entwicklung der Tennistechnik über 3 Messzeitpunkte bei Kind LH..................................165

Abbildung 37: Entwicklung der Tennistechnik über 3 Messzeitpunkte bei Kind LR ….............................165

Abbildung 38: Entwicklung der Tennistechnik über 3 Messzeitpunkte bei Kind LW................................166

Abbildung 39: Entwicklung der Tennistechnik über 3 Messzeitpunkte bei Kind LK...............................166

Abbildung 40: Entwicklung der Tennistechnik über 3 Messzeitpunkte bei Kind MAS ............................167

Abbildung 41: Entwicklung der Tennistechnik über 3 Messzeitpunkte bei Kind MS................................167

Abbildung 42: Entwicklung der Tennistechnik über 3 Messzeitpunkte bei Kind MF...............................167

Abbildung 43: Entwicklung der Tennistechnik über 3 Messzeitpunkte bei Kind MW..............................168

Abbildung 44: Entwicklung der Tennistechnik über 3 Messzeitpunkte bei Kind NM...................................168

Abbildung 45: Entwicklung der Tennistechnik über 3 Messzeitpunkte bei Kind NT ...............................169

Abbildung 46: Entwicklung der Tennistechnik über 3 Messzeitpunkte bei Kind NV.................................169

Abbildung 47: Entwicklung der Tennistechnik über 3 Messzeitpunkte bei Kind PH.............................170

Abbildung 48: Entwicklung der Tennistechnik über 3 Messzeitpunkte bei Kind SK................................170

Abbildung 49: Entwicklung der Tennistechnik über 3 Messzeitpunkte bei Kind SL..................................171

Abbildung 50: Entwicklung der Tennistechnik über 3 Messzeitpunkte bei Kind SP..............................171

Abbildung 51: Entwicklung der Tennistechnik über 3 Messzeitpunkte bei Kind TB.................................172

Abbildung 52: Entwicklung der Tennistechnik über 3 Messzeitpunkte bei Kind TH..................................172

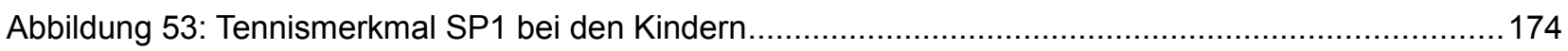

Abbildung 54: Tennismerkmal SP2 bei den Kindern ................................................................... 174

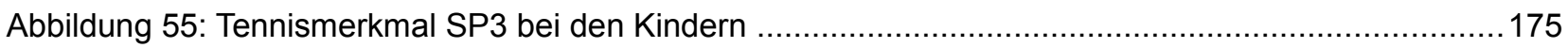

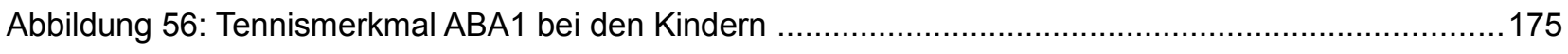

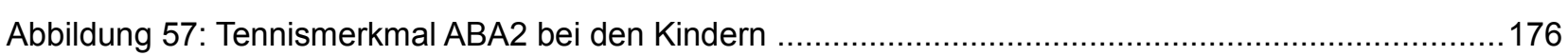

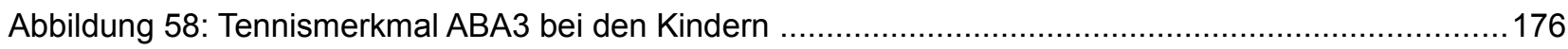

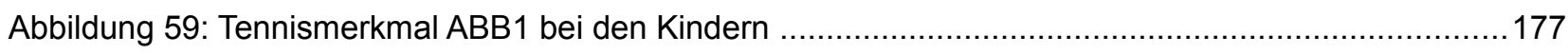

Abbildung 60: Tennismerkmal ABB2 bei den Kindern .................................................................. 177

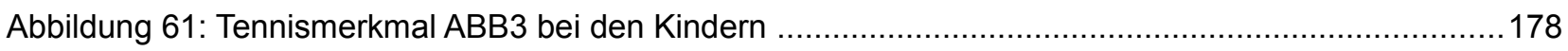

Abbildung 62: Tennismerkmal SBG1 bei den Kindern .............................................................. 178

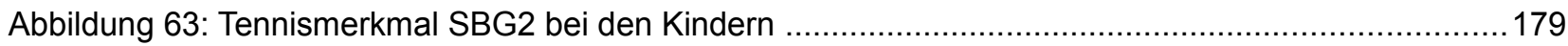

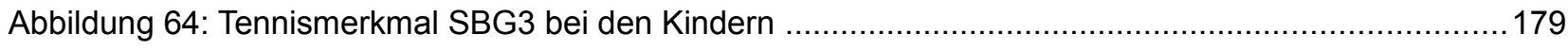

Abbildung 65: Tennismerkmal SBT1 bei den Kindern ................................................................ 180

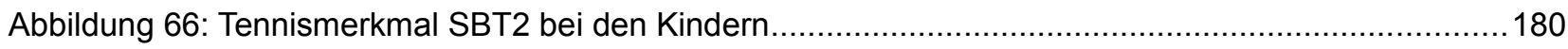

Abbildung 67: Tennismerkmal SBT3 bei den Kindern............................................................... 181

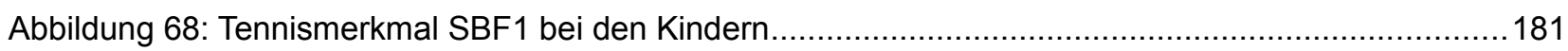

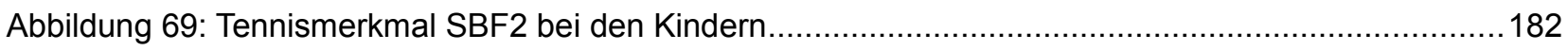

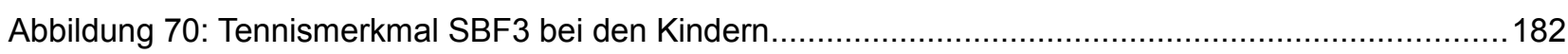

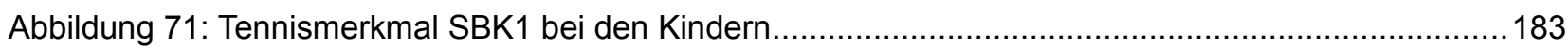


Abbildung 72: Tennismerkmal SBK2 bei den Kindern 183

Abbildung 73: Tennismerkmal SBK3 bei den Kindern 184

Abbildung 74: Tennismerkmal SBGK1 bei den Kindern 184

Abbildung 75: Tennismerkmal SBGK2 bei den Kindern 185

Abbildung 76: Tennismerkmal SBGK3 bei den Kindern. 186

Abbildung 77: Tennismerkmal SBVA1 bei den Kindern 186

Abbildung 78: Tennismerkmal SBVA3 bei den Kindern 187

Abbildung 79: Tennismerkmal SBVA2 bei den Kindern 187

Abbildung 80: Tennismerkmal SBW1 bei den Kindern 187

Abbildung 81: Tennismerkmal SBW2 bei den Kindern. 188

Abbildung 82: Tennismerkmal SBW3 bei den Kindern. 188

Abbildung 83: Tennismerkmal AB1 bei den Kindern 189

Abbildung 84: Tennismerkmal AB2 bei den Kindern 189

Abbildung 85: Tennismerkmal AB3 bei den Kindern. 190

Abbildung 86: Tennismerkmal BK1 bei den Kindern 190

Abbildung 87: Tennismerkmal BK2 bei den Kindern 191

Abbildung 88: Tennismerkmal BK3 bei den Kindern 191 


\subsection{Abkürzungsverzeichnis}

Abkürzungen für den „Fragebogen zur Beherrschung der Tennistechnik“:

SP: rechtzeitiges Einnehmen der Schlagposition

ABA: rechtzeitiger Beginn der Ausholbewegung

ABB: rechtzeitiger Beginn der Schlagphase

SBG: Griffhaltung

SBT: Treffpunkt

SBF: Fußstreckung

SBK: Kniestreckung

SBGK: Ganzkörperstreckung

SBVA: Vorwärts-Aufwärts des Schlägerkopfes

SBW: Wischbewegung

AB: flüssiges Durchschwingen/kein Auffangen

BK: Bewegungskonstanz

sonstige Abkürzungen:

„0“ Basis- bzw. Wahrnehmungsbedingung im Versuch ohne Flächenabdeckung

„50z“ Vorstellungsbedingung mit 50\% zusammenhängend abgedeckter Fläche

„50g“ Vorstellungsbedingung mit 50\% unzusammenhängend abgedeckter Fläche

MZP: Messzeitpunkt

KZG: Kurzzeitgedächtnis

LZG: Langzeitgedächtnis

VG: Versuchsgruppe

KG: Kontrollgruppe

GRUPPE-VG: anhand des Altersmittels geteilte Versuchsgruppe

MIN2_3: Differenz von zwei Messzeitpunkten (MZP 2 minus MZP 3)

KB: Kasten-Bumerang-Lauf

Kap.: Kapitel

Abb.: Abbildungen 


\subsection{Literaturverzeichnis}

Abernethy, B. (1990). Expertise, visual search and information pick-up in squash. Perception, 19, 63-77.

Aglioti, S. M., Cesari, P., Romani, M. \& Urgesi, C. (2008). Action anticipation and motor resonance in elite basketball players. Nature Neuroscience, 11, 1109-1116.

Annett, J. (1995). Motor imagery - perception or action? Neuropsychologia, 33, 13951417.

Atienza, F. L., Balaguer, I. \& Barcía-Merita, M. L. (1998). Video modeling and imaging training on performance of tennis service of 9- to 12-year-old children. Perceptual and Motor Skills, 87, 519-529.

Attneave, F. (1962). Perception and related areas. In S. Koch (Ed.), Psychology: A study of science, Vol. 4: Biologically oriented fields: Their place in psychology and in biological science (619-659). New York: McGraw-Hill.

Baddeley, A. D. (1976). The Psychology of Memory. New York: Basic Books.

Baddeley, A. D. (2012). Working memory: Theories, models, and controversies. Annual Review of Psychology, 63, 1-29.

Beck, J. \& Bös, K. (1995). Normwerte motorischer Leistungsfähigkeit. Köln: Sport und Buch Strauß.

Beni, R. de \& Pazzaglia, F. (1978). Memory for different kinds of mental images: Role of contextual and autobiographic variables. Neuropsychologia, 11, 1359-1371.

Bennett, C. M., Baird, A. A., Miller, M. B. \& Wolford, G. L. (2009, June). Neural correlates of interspecies perspective taking in the post-mortem Atlantic Salmon: an argument for multiple comparisons correction, 15th Annual Meeting of the Organization for Human Brain Mapping, San Francisco, CA.

Bennett, C. \& Miller, M. (2010). How reliable are the results from functional magnetic 201 
resonance imaging? Annals of the New York Academy of Sciences, 1191, 133-55.

Bertenthal, B. I. (1996). Origins and early development of perception, action, and representation. Annual Review of Psychology, 47, 431-459.

Bertenthal, B. I. \& Clifton, R. K. (1998). Perception and action. In D. Kuhn, R. S. Siegler (Ed.), Handbook of Child Psychology, Vol 2: Cognition, Perception, and Language (51102). New York: Wiley.

Bischoff, M., Zentgraf, K., Lorey, B., Pilgramm, S., Balser, N., Baumgartner, E., Hohmann, T., Stark, R., Vaitl, D. \& Munzert, J. (2012). Motor familiarity: Brain activation when watching kinematic displays of one's own movements. Neuropsychologia, 50 (8), 2085-2092.

Bisiach, E. \& Luzzatti, C. (1978). Unilateral neglect of representational space. Cortex, 14, 129-133.

Bohan, M., Pharmer, J. A. \& Stokes, A. F. (1999). When does imagery practice enhance performance on a motor task? Perceptual and Motor Skills, 88 (2), 651-658.

Bortz, J. (1993). Statistik für Sozialwissenschaftler (4. Aufl.). Berlin, Heidelberg, New York, London, Paris, Tokyo, Hong Kong, Barcelona, Budapest: Springer-Verlag.

Bortz, J. \& Döring, N. (2006). Forschungsmethoden und Evaluation. Heidelberg: Springer Verlag.

Bös, K. (Hrsg.) (2001). Handbuch Motorischer Tests. Göttingen: Hogrefe Verlag.

Bös, K. \& Wohlmann, R. (1987). Allgemeiner sportmotorischer Test (AST 6-11) zur Diagnose der konditionellen und koordinativen Leistungsfähigkeit. Lehrhilfen für den Sportunterricht, (36), 10, 145-156.

Bosshardt, H.-G. (1973). Der Einfluss von Vorstellungen auf Wahrnehmen und Behalten. Dissertation an der Ruhr-Universität Bochum. 
Brooks, L. R. (1968). Spatial and verbal components of the act of recall. Canadian Journal of Psychology, 22, 349-368.

Buccino, G., Binkofski, F., Fink, G. R., Fadiga, L., Fogassi, L., Gallese, V., Seitz, R. J., Zilles, K., Rizzolatti, G. \& Freund, H.-J. (2001). Action observation activates premotor and parietal areas in a somatotopic manner: an fMRI study. European Journal of Neuroscience, 13, 400-404.

Buccino, G., Lui, F., Canessa, N., Patteri, I., Lagravinese, G., Benuzzi, F., Porro, C. A. \& Rizzolatti, G. (2004). Neural circuits involved in the recognition of actions performed by nonconspecifics: An fMRI study. Journal of Cognitive Neuroscience, 16, 114-126.

Buether, A. (2010). Die Bildung der räumlich-visuellen Kompetenz. Nr. 23 der Schriftenreihe. Burg Giebichenstein Kunsthochschule Halle.

Burg, A. (1966). Visual acuity as measured by dynamic and static tests. Journal of Applied Psychology, 50, 460-466.

Burg, A. (1968). Lateral visual field as related to age and sex. Journal of Applied Psychology, 52, 10-15.

Caeyenberghs, K., Tsoupas, J, Wilson, P. H. \& Smiths-Engelsman, B. C. M. (2009a). Motor imagery development in primary school children. Developmental Neuropsychology, 34 (1), 103-121.

Caeyenberghs, K., Wilson, P. H., van Roon, D., Swinnen, S. P. \& Smits-Engelsman, B. C. (2009b). Increasing convergence between imagined and executed movement across development: evidence for the emergence of movement representations. Developmental Science, 12 (3), 474-83.

Calvo-Merino, B., Glaser, D. E., Grèzes, J., Passingham, R. E. \& Haggard, P. (2005). Action observation and acquired motor skills: an fMRI study with expert dancers. Cerebral Cortex, 15, 1243-1249.

Calvo-Merino, B., Grèzes, J., Glaser, D. E., Passingham, R. E. \& Haggard, P. (2006). 
Seeing or doing? Influence of visual and motor familiarity in action observation.

Current Biology, 16, 1905-1910.

Casile, A. \& Giese, M. A. (2006). Non-visual motor learning influences the recognition of biological motion. Current Biology, 16(1), 69-74.

Chevalier, N., Monnier, E. \& Auger, R. (1995). L'image visuelle des mouvements corporels de l'enfant de 6 ans. Revue des sciences et techniques des activités physiques et sportives, 36, 57-66.

Clarkson-Smith, L. \& Halpern, D. F. (1983). Can age-related deficits in spatial memory be attenuated through the use of verbal coding? Experimental Aging Research, 9, 179-184.

Collins, D. W. \& Kimura, D. (1997). A large sex difference on a two-dimensional mental rotation task. Behavioral Neuroscience, 111, 845-849.

Cooper, L.A. \& Shepard, R.N. (1973). Chronometric studies of the rotation of mental images. In Chase, W.G. (Ed.), Visual information processing. Academic Press, New York.

Courtine, G., Papaxanthis, C., Gentili, R. \& Pozzo, T. (2004). Gait-dependent motor memory facilitation in covert movement execution. Cognitive Brain Research, 22, 67-75.

Crammond, D. J. (1997). Motor Imagery: Never in Your Wildest Dream. Trends in Neuroscience, (20-2), 54-57.

Currie, G. \& Ravenscroft, I. (1997). Mental Simulation and Motor Imagery. Philosophy of Science, 64, 161-180.

Daugs, R. \& Blischke, K. (1984). Sensomotorisches Lernen. In K. Carl, D. Kaiser, H. Mechling \& W. Preysing (Hrsg.), Handbuch Sport, Bd. 1 (381-420). Düsseldorf: Schwann.

Dean, G. \& Morris, P. E. (1991). Imagery and spatial ability: When introspective reports predict performance. In R.H. Logie, M. Denis (Ed.), Mental images in human cognition (331-347). Amsterdam: Elsevier. 
Dean, G. M. \& Morris, P. E. (2003). The relationship between self-reports of imagery and spatial ability. British Journal of Psychology, 94, 245-273.

Decety, J. (1996). Do imagined and executed actions share the same neural substrate? Cognitive Brain Research, 3, 87-93.

Decety, J. \& Grezes, J. (1999). Neural mechanisms subserving the perception of human actions. Trends in Cognitive Science, 3 (5), 172-178.

Decety, J., Grezes, J., Costes, N., Perani, D., Jeannerod, M., Procyk, E., Grassi, F. \& Fazio, F. (1997). Brain activity during observation of actions. Influence of action content and subject's strategy. Brain, 120, 1763-1777.

Decety, J., Jeannerod, M. \& Prablanc, C. (1989). The timing of mentally represented actions. Behavioral Brain Research, 34 (1-2), 35-42.

Decety, J., Sjoholm, H., Ryding, E., Stenberg, G. \& Ingvar, D. (1990). The cerebellum participates in cognitive activity: Tomographic measurements of regional cerebral blood flow. Brain Research, 535, 313-317.

Denis, M. (1985). Visual imagery and the use of mental practice in the development of motor skills. Canadian Journal of Applied Sport Science, 10, 4-16.

Dominey, P., Decety, J., Brouselle, E., Chazot, G. \& Jeannerod, M. (1995). Motor imagery of a lateralized sequential task is asymmetrically slowed in hemi-Parkinson's patients., Neuropsychologia, 33, 727-741.

Driskell J. E., Copper C. \& Moran A. (1994). Does mental practice enhance performance? Journal of Applied Psychology, 79, 481-492.

Dültgen, K., Munzert, J. \& Möllmann, H. (1996). Erfassung sportspezifischer Vorstellungskompetenzen junger Badmintonspieler- und -spielerinnen. In R. Daugs, K. Blischke, F. Marschall \& H. Müller (Hrsg.), Kognition und Motorik (S. 147-152). Hamburg: Czwalina. 
Eals, M. \& Silverman, I. (1994). The hunter-gatherer theory of spatial sex differences: Factors mediating the female advantage in location memory. Ethology and Sociobiology, 15, 95-105.

Eisler, R. (1904). Wörterbuch der philosophischen Begriffe. Apperzeption. URL: http://www.textlog.de/1381.html [Zugriff: 4.4.13].

Elliott, D. \& Maraj, B. K. V. (1994). Visual-spatial movement goals. Behavioral and Brain Sciences, 17, 207.

Engelkamp, J. \& Zimmer, H. D. (2006). Lehrbuch der kognitiven Psychologie. Göttingen: Hogrefe.

Estes, D. (1998). Young children's awareness of their mental activity: The case of mental rotation. Child Development, 69 (5), 1345-1360.

Eysenck, M. W. (2012). Fundamentals of cognition. ( $2^{\text {nd }}$ ed.). Hove East Sussex [u.a.]: Psychology Press. URL: http://www.psypress.com/fundamentals-of-cognition-2ndedition-9781848720718 [Zugriff: 9.4.13].

Farah, M. J. (1984). The Neurological Basis of Mental Imagery: A Componential Analysis. Cognition, 18, 245-72.

Farah, M. J., Soso, M. J. \& Dasheiff, R. M. (1992). Visual angle of the mind's eye before and after unilateraloccipital lobectomy. Journal of Experimental Psychology: Human Perception and Performance, 18, 241-246.

Feltz, D. L. \& Landers, D. M. (1983). The effects of mental practice on motor skill learning and performance: A meta-analysis. Journal of Sport Psychology, 5, 25-57.

Féry Y. A. (2003). Differentiating visual and kinesthetic imagery in mental practice. Canadian Journal of Experimental Psychology, 57, 1-10.

Finke, R. A. (1979). The functional equivalence of mental images and errors of movement. Cognitive Psychology, 11, 235-264. 
Finke, R. A. (1980). Levels of equivalence in imagery and perception. Psychological Review, 87, 113-132.

Finke, R. A. (1986). Bildhaftes Vorstellen und visuelle Wahrnehmung. In M. Ritter (Hrsg.), Wahrnehmung und visuelles System (S. 178-185). Heidelberg: Spektrum der Wissenschaft.

Fitts, P. M. (1954). The information capacity of the human motor system in controlling the amplitude of movement. Journal of Experimental Psychology, 47 (6), 381-391.

Fox, P. T., Pardo, J. V., Petersen, S. E. \& Raichle, M. E. (1987). Supplementary motor and premotor responses to actual and imagined hand movements with positron emission tomography. Neuroscience Abstracts, 13, 1433.

Frester, R. (1996). Interne Repräsentation und Qualität der Bewegungsausführung. In R. Daugs, K. Blischke, F. Marschall, H. Müller (Hrsg.), Kognition und Motorik (161-166). Hamburg: Czwalina.

Frick, A., Daum, M. M., Wilson, M. \& Wilkening, F. (2009). Effects of action on children's and adults' mental imagery. Journal of Experimental Child Psychology, 104 (1), 34-51.

Funk, M., Brugger, P. \& Wilkening, F. (2005). Motor processes in children's imagery: the case of mental rotation of hands. Developmental Science, 8 (5) , 402-408.

Gabbard, C. (2012). The role of mental simulation in embodied cognition. Early Child Development and Care, 1-8, iFirst Article.

Ganis, G., Thompson, W. L. \& Kosslyn, S. M. (2004). Brain areas underlying visual mental imagery and visual perception: An fMRI study. Cognitive Brain Research, 20, 226241.

Gazzaniga, M. S., Ivry, R. B. \& Mangun, G. R. (2009). Cognitive neuroscience: The biology of the mind $\left(2^{\text {nd }}\right.$ ed.). New York: W. W. Norton. 
Ginsburg, N., Jurenovskis, M. \& Jamieson, J. (1982). Sex differences in critical flicker frequency. Perceptual and motor skills, 54, 1079-1082.

Goldenberg, G., Podreka, I., Steiner, M., Willmes, K., Suess, E. \& Deecke, L. (1989a). Regional cerebral blood flow patterns in visual imagery. Neuropsychologia, 27, 641-664.

Goldenberg, G., Podreka, I., Uhl, F., Steiner, M., Willmes, K., Suess, E. \& Deecke, L. (1989b). Cerebral correlates of imaging colours, faces and a map. I. SPECT of regional cerebral blood flow. Neuropsychologia, 27, 1315-1328.

Golledge, R. (1999). Human Wayfinding and Cognitive Maps. In R. Golledge (Ed.), Wayfinding Behavior - Cognitive Mapping and Other Spatial Processes (5-45). Baltimore: Johns Hopkins University Press.

Goodale, M. A. \& Milner, M. A. (2004). Sight Unseen: An Exploration of Conscious and Unconscious Vision. Oxford: Oxford University Press.

Grouios, G. (1992). Mental practice: A review. Journal of Sport Behavior, 15, 42-59.

Guillot, A \& Collet, C. (2005). Duration of mentally simulated movement: a review. Journal of Motor Behavior, 37 (1), 10-20.

Hacker, W. (1998). Allgemeine Arbeitspsychologie. Psychische Regulation von Arbeitstätigkeiten. Bern, Göttingen, Toronto, Seattle: Verlag Hans Huber.

Hahn, N. (2010). Mentale Rotation bei Vorschulkindern: Geschlechtsunterschiede in der Lateralisierung. Dissertation an der Heinrich-Heine-Universität Düsseldorf.

URL: $\quad$ http://docserv.uni-duesseldorf.de/servlets/DerivateServlet/Derivate17865/Diss_final_Nicky\%20\%28pdfA\%29.pdf [Zugriff: 1.11.11].

Hahn, N., Jansen P. \& Heil, M. (2009). Preschoolers' mental rotation: sex differences in hemispheric asymmetry. Journal of Cognitive Neuroscience, 22 (6), 1244-1250.

Hall, C. \& Martin, K. E. (1997). Measuring movement imagery abilities: a revision of the Movement Imagery Questionnaire. Journal of Mental Imagery, 21, 143-154. 
Hall, C., Pongrac, J. \& Buckolz, E. (1985). The measurement of imagery ability. Human Movement Science, 4 (2), 107-118.

Halpern, D. F. (1986). Sex Differences in Cognitive Abilities. Hillsdale, N.J.: Erlbaum.

Hamilton A, Wolpert, D. M. \& Frith, U. (2004). Your own action influences how you perceive another person's action. Current Biology, 14, 493-98.

Heil, M. \& Jansen-Osmann, P. (2008a). Sex differences in mental rotation with polygons of different complexity: Do men utilize holistic processes whereas women prefer piecemeal ones? The Quarterly Journal of Experimental Psychology, 61 (5), 683-689.

Heil, M. \& Jansen-Osmann, P. (2008b). Gender differences in math and mental rotation accuracy but not in mental rotation speed in 8-years-old children. European Journal of Developmental Science, 2, 190-196.

Hinshaw, K. E. (1991). The effects of mental practice on motor skill performance: Critical evaluation and metaanalysis. Imagination, Cognition and Personality, 11, 3-35.

Hirsch, O., Lehmann, W., Corth, M., Röhrle, B., Schmitz, S. \& Schipper, H.I. (2003). Visuelle Vorstellungsfähigkeit, Bewegungsvorstellung und mentales Rotieren bei Morbus Parkinson. Zeitschrift für Neuropsychologie, 14 (2), 67-80.

Hohmann, T., Olmos, A. \& Munzert, J. (2011). The influence of motor expertise and motor experience on action and actor recognition. Journal of Cognitive Psychology, 23 (4), 403415.

Holling, H. \& Schmitz, B. (Hrsg.) (2010). Handbuch Statistik, Methoden und Evaluation. Göttingen: Hogrefe.

Hommel, B., Müsseler, J., Aschersleben, G. \& Prinz, W. (2001). The theory of event coding (TEC): A framework for perception and action planning. Behavioral and Brain Sciences, 24, 849-878. 
Hoyek, N., Champely, S., Collet, C., Fargier, P. \& Guillot, A. (2009). Age and genderrelated differences in the temporal congruence development between motor imagery and motor performance. Learning and Individual Differences, 19 (4), 555-560.

Huber, J. (1997). Differences in problem representation and procedural knowledge between elite and nonelite springboard divers. The Sport Psychologist, 11 (2), 142-159.

Huys, R., Cãnal-Bruland, R., Hagemann, N., Beek, P. J., Smeeton, N. J.\& Williams, A. M. (2009). Global information pickup underpins anticipation of tennis shot direction. Journal of Motor Behavior, 41, 158-170.

Ingvar, D.\& Philipsson, L. (1977). Distribution of the cerebral blood flow in the dominant hemisphere during motor ideation and motor performance. Annals of Neurology, 2, 230237.

Isaac, A. R.\& Marks, D. F. (1994). Individual differences in mental imagery experience: Developmental changes and specialization. British Journal of Psychology, 85, 479-500.

Isaac, A., Marks, D. F. \& Russell, D. G. (1986). An instrument for assessing imagery of movement: The Vividness of Movement Imagery Questionnaire (VMIQ). Journal of Mental Imagery, 10 (4), 23-30.

Ishai, A. (2010). Seeing with the mind's eye: top-down, bottom-up, and conscious awareness. Biology Reports, 2, 34. doi: 10.3410/b2-34, URL:

http://f1000.com/reports/biology/content/2/34 [Zugriff: 24.4.2013].

Ishai, A. \& Sagi, D. (1997a). Visual imagery facilitates visual perception: Psychophysical evidence. Journal of Cognitive Neuroscience, 9 (4), 476-489.

Ishai, A. \& Sagi, D. (1997b). Visual imagery: Effects of short- and long-term memory. Journal of Cognitive Neuroscience, 9 (6), 734-742. 
Ishai, A. \& Sagi, D. (1998). Visual imagery and visual perception: The role of memory and conscious awareness. In S. Hameroff, A. Kaszniak \& A. Scott (Eds.), Toward a science of consciousness II (321-328). MIT Press.

URL: http://www.weizmann.ac.il/home/masagi//shaiSagi_tucson98.pdf [Zugriff: 8.5.13]

Jackendoff, R. \& Landau, B. (1994). What is coded in parietal representations? Behavioral and Brain Sciences, 17, 211-212.

Jacobs, A.\& Shiffrar, M. (2005). Walking perception by walking observers. Journal of Experimental Psychology: Human Perception and Performance, 31, 157-169.

Jacobson, E. (1930). Electrical measurements of neuromuscular states during mental activities. American Journal of Physiology, 96, 115-121.

James, W. (1890). Principles of Psychology. URL: http://psychclassics.asu.edu/James/Principles/prin26.htm [Zugriff: 21.03.2012].

Jansen, P. \& Heil, M. (2010). The relation between motor development and mental rotation ability in 5- to 6-year-old children. European Journal of Developmental Science, 4 (1), 67-75.

Jaspers, K. (1965). Allgemeine Psychopathologie (8. Aufl.). Heidelberg: Springer Verlag.

Jeannerod, M. (1994). The representing brain: Neural correlates of motor intention and imagery. Behavioral and Brain Sciences, 17, 187-245.

Jeannerod M. (1995). Mental imagery in the motor context. Neuropsychologia. 33 (11), 1419-32.

Jeannerod M. (2001). Neural simulation of action: A unifying mechanism for motor cognition. Neurolmage, 14, 103-109.

Jeannerod, M. (2006). Motor cognition. What actions tell the Self. Oxford: Oxford University Press. 
Johansson, G. (1973). Visual perception of biological motion and a model for its analysis. Perception and Psychophysics, 14 (2), 201-211.

Johnson, P. (1982). The functional equivalence of imagery and movement. Quarterly Journal of Experimental Psychology, 34a, 349-365.

Jordan, K., Wüstenberg, T., Heinze, H.-J., Peters, M. \& Jäncke, L. (2002). Women and men exhibit different cortical activation patterns during mental rotation tasks. Neuropsychologia, 40, 2397-2408.

Kail, R., Pellegrino, J. \& Carter, P. (1980). Developmental changes in mental rotation. Journal of Experimental Child Psychology, 29, 102-116.

Karádi, K., Szabo, I., Szepesi, T., Kallai, J. \& Kovacs, B. (1999). Sex differences on the hand mental rotation task for 9-yr.-old children and young adults. Perceptual and Motor Skills, 89, 969-972.

Kebeck, G. (1997). Wahrnehmung: Theorien, Methoden und Forschungsergebnisse der Wahrnehmungspsychologie (2. Aufl.). Weinheim, München: Juventa Verlag.

Keller, P. E., Knoblich, G. \& Repp, B. H. (2007). Pianists duet better when they play with themselves: On the possible role of action simulation in synchronization. Consciousness and Cognition, 16, 102-111.

Kerr, N. H. (1983). The role of vision in "visual imagery" experiments. Evidence from the congentially blind. Journal of Experimental Psychology. General, 112, 265-277.

Kim, J., Singer, R. N. \& Tennant, L. K. (1998). Visual, auditory, and kinaesthetic imagery on motor learning. Journal of Human Movement Studies, 5, 187-210.

Kimura, D. (1999). Sex and Cognition. Cambridge (Mass.), London (England): MIT Press.

Kioumourtzoglou, E., Kourtessis, T., Michalopoulou, M. \& Derri, V. (1998). Differences in several perceptual abilities between experts and novices in basketball, volleyball 
and water polo. Perceptual and Motor Skills, 86, 899-912.

Klatzky, R. L. (1994). On the relation between motor imagery and visual imagery. Behavioural and Brain Sciences, 17, 212-213.

Knoblich, G. \& Flach, R. (2001). Prediction the effects of actions: Interactions of perception and action. Psychological Science, 12 (6), 467-472.

Koffka, K. (1935). Principles of Gestalt psychology. New York: Harcourt, Brace.

Kohen, R. (1954). Intelligenz und Bewegungsvorstellung. Dissertation. Phil. I, Université de Zurich.

Köhler, W. (1929). Gestalt psychology. New York: Liveright.

Kosslyn, S. M. (1980). Image and Mind. Cambridge, MA: Harvard University Press.

Kosslyn, S. M. (1994). Image and Brain: The Resolution of the Imagery Debate. Cambridge, MA: MIT Press.

Kosslyn, S. M. (2005). Mental images and the brain. Cognitive Neuropsychology, 22, 333-347.

Kosslyn, S. M., Behrmann, M. \& Jeannerod, M. (1995). The cognitive neuroscience of mental imagery. Neuropsychologia, 33 (11), 1335-1344.

Kosslyn, S. M., Ganis, G. \& Thompson, W. L. (2001) Neural foundations of imagery. Nature Reviews Neuroscience, 2, 635-642.

Kosslyn, S. M., Ganis, G. \& Thompson, W. L. (2010). Multimodal images in the brain. In A. Guillot \& C. Collet (Eds.). The Neurophysiological Foundations of Mental and Motor Imagery. Oxford: Oxford University Press.

Kosslyn, S. M., Margolis, J. A., Barrett, A. M., Goldknopf, E. J. \& Daly, P. F. (1990). Age differences in imagery abilities. Child Development, 61, 995-1010. 
Kosslyn, S. M. \& Thompson, W. L. (2003). When is early visual cortex activated during visual mental imagery? Psychological Bulletin, 129 (5), 723-746.

Kosslyn, S.M., Thompson, W. L. \& Ganis, G. (2006). The Case for Mental Imagery. New York, N.Y. [u.a.]: Oxford Psychology Series.

Kosslyn, S. M., Thompson, W. L., Kim, I. J. \& Alpert, N. M. (1995). Topographical representations of mental images in primary visual cortex. Nature, 378, 496-498.

Léonard, G. \& Tremblay, F. (2007). Corticomotor facilitation associated with observation, imagery and imitation of hand actions: a comparative study in young and old adults. Experimental Brain Research, 177, 167-175.

Lequerica, A., Rapport, L., Axelrod, B. N., Telmet , K. \& Whitman, R. D. (2002). Subjective and objective assessment methods of mental imagery control: Construct validations of self-report measures. Journal of Clinical and Experimental Neuropsychology, 24 (8), 1103-1116.

Levine, S. C., Huttenlocher, J., Taylor, A. \& Langrock, A. (1999). Early sex differences in spatial skill. Developmental Psychology, 35, 940-949.

Linn, M. C. \& Petersen, A. C. (1985). Emergence and characterization of sex differences in spatial ability: A meta-analysis. Child Development, 56, 1479-1498.

Livesey, D. J. (2002). Age differences in the relationship between visual movement imagery and performance on kinesthetic acuity tests. Developmental Psychology, 38 (2), 279-287.

Logie, R. H. (1995). Visual-spatial working memory. Hove, UK: Erlbaum.

Lorey, B., Bischoff, M., Pilgramm, S., Stark, R., Munzert, J. \& Zentgraf, K. (2009). The embodied nature of motor imagery: The influence of posture and perspective. Experimental Brain Research, 194, 233-243. 
Manidi-Faes, M.-J. (1996). Relation entre action et représentation cognitive d'une figure gymnique: effets de l'age et de la repetetion. Science et techniques des activites physiques et sportives, 17 (40), 77-86.

Marey, E. J. (1895/1972). Movement. New York: Arno Press and New York Times.

Marks, D. F. (1973). Visual imagery differences in the recall of pictures. British Journal of Psychology, 64, 17-24.

Marmor, G. S. (1975). Development of kinetic images: when does the child first represent movement in mental images? Cognitive Psychology, 7, 548-559.

Marr, D. (1982). Vision: A computational investigation into the human representation and processing of visual information. San Francisco: W. H. Freeman.

Mast, F. W. (2005). Mit dem inneren Auge sehen - Wie hängen Wahrnehmung und Vorstellung zusammen? Neuroforum, 3, 80-87.

McCarron, L. T. (1997). MAND McCarron assessment of neuromuscular development: Fine and gross motor abilities (revised ed.). Dallas, TX: Common Market Press.

McGee, M. G. (1979). Human spatial abilities: Psychometric studies and environmental, genetic, hormonal, and neurological influences. Psychological Bulletin, 86, 889-918.

McGinn, C. (2007). Das geistige Auge. Von der Macht der Vorstellungskraft. Darmstadt: Primus Verlag.

Miller, F. P., Vandome, A. F. \& McBrewster, J. (Eds.). (2010). Motor imagery. Alphascript Publishing.

Milner, A. D. \& Goodale, M. A. (1995). The Visual Brain in Action. Oxford: Oxford University Press.

Milner, A. D. \& Goodale, M. A. (1998). The visual brain in action. Psyche, 4, 1-14. 
Milner, A. D. \& Goodale, M. A. (2008). Two visual systems re-viewed. Neuropsychologia, 46, 774-785.

Mishkin, M., Ungerleider, L. G. \& Macko, K. A. (1983). Object vision and spatial vision: two cortical pathways. Trends in Neurosciences, 6, 414-417.

Molina, M., Tijus, C. \& Jouen, F. (2008). The emergence of motor imagery in children. Journal of Experimental Child Psychology, 99 (3), 196-209.

Moran, A. P. (2004). Sport and exercise psychology: a critical introduction. New York: Routledge.

Mulder, T., Zijlstra, S., Zijlstra, W. \& Hochstenbach, J. (2004). The role of motor imagery in learning a totally novel movement. Experimental Brain Research, 154, 211-217.

Munzert, J. (1996). Ein computationaler Ansatz zur Differenzierung von (Bewegungs-) Vorstellungen. In E. Loosch, M. Tamme (Hrsg.), Motorik- Struktur und Funktion- 4. Symposium der dvs-Sektion Sportmotorik vom 25.-27-01.1996 in Erfurt. (S. 113-117). Hamburg: Czwalina.

Munzert, J. (2001a). Bewegungsvorstellungen - Bewegungshandlungsvorstellungen. In D. Hackfort (Hrsg.). Handlungspsychologische Forschung für die Theorie und Praxis der Sportpsychologie (S. 49-63). Köln: bps-Verlag.

Munzert, J. (2001b). Vorstellung und Bewegung. In J.R. Nitsch, H. Allmer (Hrsg.), Denken, Sprechen, Bewegen (S. 41-56). Köln: bps-Verlag.

Munzert, J. (in press). Does level of expertise influence imagined durations in open skills? Played versus imagines durations of badminton sequences. International Journal of Sport and Exercise Psychology.

Munzert, J., Lorey, B. \& Zentgraf, K. (2009). Cognitive motor processes: The role of motor imagery in the study of motor representations. Brain Research Review, 60, 306326. 
Munzert, J. \& Reiser, M. (2003). Vorstellung und mentales Training. In H. Mechling \& J. Munzert (Hrsg.), Handbuch Bewegungswissenschaft - Bewegungslehre (S. 219-230). Schorndorf: Hofmann.

Murphy, S. M. (1994). Imagery interventions in sport. Medicine and Science in Sports and Exercise, 26 (4), 486-494.

Müsseler, J. \& Hommel, B. (1997). Blindness to response-compatible stimuli. Journal of Experimental Psychology: Human Perception and Performance, 23, 861-872.

Naito, E. (1994). Controllability of motor imagery and transformation of visual imagery. Perceptual and Motor Skills, 78(2), 479-87.

Neubauer, A. C., Bergner, S. \& Schatz, M. (2010). Two- vs. three-dimensional presentation of mental rotation tasks: Sex differences and effects of training on performance and brain activation. Intelligence, 38, 529-539.

Nishida, T., Katube, A., Inomata, K., Okazawa, Y., Ito, M, Kayama, S., Tsuruhara, K. \& Yoshizawa, Y. (1986). A new test for controllability of motor imagery: the examination of its validity and reliability. Japanese Journal of Physical Education, 31, 13-22.

Norman, D. A. (1979). Perception, memory, and mental process. In L.G. Nilson (Ed.). Perspectives on memory research. Hillsdale: Erlbaum.

Oerter, R. \& Montada, L. (1995). Entwicklungspsychologie (3. Aufl.). Weinheim: Psychologie Verlags Union.

Opwis, K. \& Lüer, G. (1996). Modelle der Repräsentation von Wissen. In D. Albert, H. Stapf (Hrsg.), Enzyklopädie der Psychologie. Gedächtnis (Themenbereich Theorie und Forschung; Serie I/ Kognition, Band 4). Göttingen: Hogrefe.

Orliaguet, J. P. \& Coello, Y. (1998). Differences between actual and imagined movements: A chronometricanalysis. International Journal of Sport Psychology, 29,157169. 
Paivio, A. (1986). Mental representations. A dual coding approach. Clarendon Press, Oxford.

Palmer, S. E. (1978). Fundamental aspects of cognitive representation. In E. Rosch, B. Lloyd (Eds.), Cognition and categorization. Hillsdale, NJ: Erlbaum.

Papaxanthis, C., Pozzo, T., Kasprinski R. \& Berthoz, A . (2003): Comparison of actual and imagined execution of whole-body movements after a long exposure to microgravity. Neuroscience Letters, 339 (1), 41-44.

Pearson, J., Clifford, C. W. G. \& Tong, F. (2008). The functional impact of mental imagery on conscious perception. Current Biology, 18, 982-986.

Perky, C. (1910). An experimental study of imagination. American Journal of Psychology, $21,422-452$.

Piaget, J. (1954). The construction of reality in the child. New York: Basic Books.

Piaget, J. \& Inhelder, B. (1971). Mental imagery in the child. London: Routledge \& Kegan Paul. (Original veröffentlicht als: L'image mentale chez l'enfant. Presses Universitaires de France, 1966.)

Porro, C. A., Francescato, M. P. , Cettolo, V., Diamond, M. E., Baraldi, P., Zuiani, C., Bazzocchi, M. \& di Prampero, P. E. (1996) Primary motor and sensory cortex activation during motor performance and motor imagery: a functional magnetic resonance imaging study. The Journal of Neuroscience, 16 (23), 7688-7698.

Prinz, W. (1984). Attention and sensitivity in visual search. Psychological Research, 45, 355-366.

Prinz, W. (1994). Motor images are action plans (Commentary on Jeannerod, 1994). Behavioral and Brain Sciences, 17, 218.

Prinz, W. (1997). Perception and action planning. European Journal of Cognitive Psychology, 9, 129-154. 
Prinz, W. \& Bridgeman, B. (Hrsg.). (1994). Enzyklopädie der Psychologie (Themenbereich C Theorie und Forschung, Serie II Kognition, Band 1 Wahrnehmung). Göttingen: Hogrefe.

Proffitt, D. R., Bhalla, M., Gossweiler, R. \& Midgett, J. (1995). Perceiving geographical slant. Psychonomic Bulletin and Review, 2, 409-428.

Pylyshyn, Z. W. (1981). The imagery debate: Analogue media versus tacit knowledge. Psychological Review, 87,16-45.

Pylyshyn, Z. W. (2002). Mental imagery: In search of a theory. Behavioral and Brain Sciences, 25, 157-238.

Reed, C. L. (2002). Chronometric comparisons of imagery to action: Visualizing versus physically performing springboard dives. Memory and Cognition, 30 (8), 1169-1178.

Reed, C. L. \& McGoldrick, J. E. (2007). Action during body perception: Processing time affects self-other correspondences. Social Neuroscience, 2, 134-149.

Richardson, A. (1969). Mental Imagery. New York: Springer.

Richardson, A. (1977). The meaning and measurement of memory imagery. British Journal of Psychology , 68 (1), 29-43.

Rieser, J. J., Garing, A. E. \& Young, M. F. (1994). Imagery, action, and your children's spatial orientation: It's not being there that counts, it's what one has in mind. Child Development, 65, 1262-1278.

Rilea, S. L. (2008). Sex and hemisphere differences when mentally rotating meaningful and meaningless stimuli. Laterality, 13, 217-233.

Rizzolatti, G., Fogassi, L. \& Gallese, V. (2001). Neurophysiological mechanisms underlying the understanding and imitation of action. Nature Reviews Neuroscience, 2, 661-670. 
Roberts, J. E. \& Bell, M. A. (2003). Two- and three-dimensional mental rotation tasks lead to different parietal laterality for men and women. International Journal of Psychophysiology, 50, 235-246.

Rockmann, U. (1998). Illustration „Große Körperwelle“. URL: http: www.unioldenburg.de/sport/bbw2/Expmente/onl_exp/kwelle/KW.html [Zugriff 2.3.2006].

Roland, P. E. \& Friberg, L. (1985). Localization of cortical areas activated by thinking. Journal of Neurophysiology, 53, 1219-1243.

Roland, P., Larsen, B., Lassen, N. \& Skinhoj, E. (1980). Supplementary motor area and other cortical areas in organization of voluntary movements in man. Journal of Neurophysiology, 43, 118-136.

Rollins, M. (2006). Mental Imagery. In Borchert, D. M. (Ed.). Encyclopedia of Philosophy. (2 ${ }^{\text {nd }}$ Ed., Vol. 4). Detroit: Macmillan.

Rosenbaum, D. A., Carlson, R. A. \& Gilmore, R. O. (2001). Aquisition of intellectual and perceptual-motor skills. Annual Review of Psychology. 52, 453-70.

Rosenzweig, M. R., Leiman, A. L. \& Breedlove, S. M. (Hrsg.). (1999). Biological Psychology: An Introduction to Behavioral, Cognitive, and Clinical Neuroscience. (2nd Ed.). Sunderland, Massachusetts: Sinauer Associates Inc.

Scherer, H.-G. (2003). Bewegungsvorstellung. In Röthig, P. \& R. Prohl (Hrsg.), Sportwissenschaftliches Lexikon (7. Aufl.), (S. 105-106). Schorndorf: Hofmann.

Sebanz, N. \& Shiffrar, M. (2009). Detecting deception in a bluffing body: The role of expertise. Psychonomic Bulletin and Review, 16, 170-175.

Sekuler, R. \& Blake, R. (2002). Perception (4 ${ }^{\text {th }}$ ed.). New York: McGraw-Hill.

Sharps, M. J., Price, J. L. \& Williams, J. K. (1994). Spatial cognition and gender: Instructional and stimulus influences on mental image rotation performance. Psychology 
of Women Quarterly, 18 (3), 413-425.

Sheikh, A. A. \& Korn, E. R. K. (1994). Imagery in sports and physical performance. New York: Baywood Publishing Company.

Shepard, R. N. \& Metzler, J. (1971). Mental rotation of three-dimensional objects. Science, 111, 701-703.

Shiffrar, M. \& Freyd, J. (1990). Apparent motion of the human body. Psychological Science, 1, 257-264.

Shiffrar, M. \& Heinen, T. (2010). Die Fähigkeiten von Athleten verändern deren Wahrnehmung von Handlungen. „Embodiment“ der visuellen Wahrnehmung von menschlichen Bewegungen. Zeitschrift für Sportpsychologie, 17 (4), 130-142.

Sirigu, A., Cohen, L., Duhamel, J. R., Pillon, B., Dubois, B., Agid, Y. \& Pierrot-Deseilligny, C. (1995). Congruent unilateral impairments for real and imagined hand movements. Neuroreport: An International Journal for the Rapid Communication of Research in Neuroscience, 6 (7), 997-1001.

Sirigu, A., Duhamel, J. R., Cohen, L., Pillon, B., Dubois, B. \& Agid, Y. (1996). The mental representation of hand movements after parietal cortex damage. Science, 273, 15641568.

Smyth, M. M. \& Waller, A. (1998). Movement imagery in rock climbing: patterns of interference from visual, spatial and kinaesthetic secondary tasks. Applied Cognitive Psychology, 12, 145-157.

Solodkin, A., Hlustik, P., Chen, E. E \& Small, S. L. (2004). Fine modulation in network activation during motor execution and motor imagery. Cerebral Cortex, 14 (11), 1246-55.

Stern, W. (1935). Allgemeine Psychologie auf personalistischer Grundlage. Bd. 1-2. Den Haag: Nijhoff.

Stevens J. A. (2005) Interference effects demonstrate distinct roles for visual and motor 
imagery during the mental representation of human action. Cognition, 95, 329-350.

Stränger, J. (1977). Beobachtungslernen: Kognitive Analyse des Erwerbs von Handlungsplänen durch intentionale Modellbildung bei elf- bis dreizehnjährigen Schülern. Unveröffentlichte Dissertation, Fakultät für Psychologie der Ruhr-Universität, Bochum.

Thomas, N. J. T., (2010). Mental Imagery. In E. N. Zalta (Ed.), The Stanford Encyclopedia of Philosophy (Standford University). URL: http://plato.stanford.edu/entries/mental-imagery/ [Zugriff: 28.03.2012].

Vecchi, T. \& Girelli, L. (1998). Gender differences in visuo-spatial processing: The importance of distinguishing between passive storage and active manipulation. Acta Psychologica, 99, 1-16.

Voyer, D., Voyer, S. \& Bryden, M. P. (1995). Magnitude of sex differences in spatial abilities: A meta-analysis and consideration of critical variables. Psychological Bulletin, 117 (2), 250-270.

Waber, D. P., Carson, D. \& Mann, M. (1982). Developmental and differential aspects of mental rotation in early adolescence. Child Development, 53, 1614-1621.

Wechsler, D. (1958). The measurement and appraisal of adult intelligence. (S. 146). Baltimore: Williams and Wilkins.

Wentura, D. \& Frings, C. (2013). Kognitive Psychologie. Wiesbaden: Springer-Verlag.

Wertheimer, M. (1923). Untersuchungen zur Lehre von der Gestalt. Psychologische Forschung: Zeitschrift für Psychologie und ihre Grenzwissenschaften, 4, 301-350.

Wertheimer, M. (1925). Drei Abhandlungen zur Gestalttheorie. Erlangen: Verlag der Philos. Akademie.

Wexler, M., Kosslyn, S. M. \& Berthoz, A. (1998). Motor processes in mental rotation. Cognition, 68, 77-94. 
Wiemann, K. (2001). Bewegungswahrnehmung und Bewegungsvorstellung im Sport. URL: http://www.biowiss-sport.de/beweg_wahr_vor.PDF [Zugriff: 04.08.2006].

Wiemeyer, J. (2001). Conscious representations of movement: Structure and assessment. In J. Munzert, S. Künzell, M., Reiser, N. Schott (Hrsg.). (2001). Bewegung, Bewusstsein, Lernen. Beiträge der dvs-Sektionssitzung Sportmotorik vom 25.-27.1.2001 in Gießen.

Wiemeyer, J. \& Angert, R. (2011). Computer methods to assess motor imagery. International Journal of Computer Science in Sport, 10 (2), 37-53.

Wilkening, F. \& Krist, H. (1995). Entwicklung der Wahrnehmung und Psychomotorik. In R. Oerter \& L. Montada. (Hrsg.), Entwicklungspsychologie (3. Aufl.), (S. 487- 517). Weinheim: Beltz Psychologie Verlags Union.

Williams, A. M. \& Davids, K. (1998). Visual search strategy, selective attention and expertise in soccer. Research Quarterly for Exercise and Sport, 69, 111-128.

Wilson, M. (2001). Perceiving imitable stimuli: Consequences of isomorphism between input and output. Psychological Bulletin, 127, 543-553.

Witkin, H. A. (1967). A cognitive style approach to cross-cultural research. International Journal of Psychology, 2, 233-250.

Wright, M. J., Bishop, D. T., Jackson, R. C. \& Abernethy, B. (2010). Functional MRI reveals expert-novice differences during sport-related anticipation. NeuroReport, 21, 9498.

Wulf, G. \& Prinz, W. (2001). Directing attention to movement effects enhances learning: A review. Psychonomic Bulletin and Review, 8 (4), 648-660.

Yue, G. \& Cole, K. (1992). Strength increases from the motor program: Comparison of training with maximal voluntary and imagined muscle contractions. Journal of Neurophysiology, 67, 114-1123. 


\subsection{Danksagung}

Diese Dissertation entstand im Rahmen eines Forschungsprojektes an der Universität Göttingen in Zusammenarbeit mit dem Niedersächsischen Tennisverband (Projektleiter waren Prof. Dr. Herbert Hopf und NTV-Vizepräsident Jörg Reinhard). An der Organisation und Durchführung haben zahlreiche Personen mitgewirkt, die zum Gelingen beigetragen haben. Zunächst möchte ich mich bei allen Eltern und Kindern für die Begleitung zum Training, die regelmäßige Teilnahme am Projekt sowie das große Interesse daran bedanken.

Die Beschäftigung mit Vorstellungen im Kontext des Erwerbs sportlicher Fertigkeiten entstand auf Anregung von Herrn Prof. Dr. Herbert Hopf im Projekt „Bewegungsrepräsentationen am Beispiel Jüngsten-Tennis“. Ihm gilt mein ausdrücklicher Dank, auch für die Diskussion der Erkenntnisse des Projekts für die Sportpädagogik und den dadurch erhaltenen Einblick in das zunächst fachfremde Gebiet.

Herrn Prof. Dr. Andreas Bund danke ich für die zahlreichen Literaturhinweise und für dessen Bereitschaft sich mit der vorliegenden Arbeit auseinanderzusetzen.

Herrn Prof. Dr. Gerd Thienes danke ich für die Bereitschaft an der Disputation mitzuwirken.

Mein besonderer Dank gilt weiterhin auch dem gesamten Projektteam sowie den studentischen Hilfskräften. Auch danke ich den Tennistrainern, die zur Entwicklung des „Fragebogens zur Erfassung der Tennistechnik“ beigetragen haben sowie den TennisExperten, die die Tennistechnik der Kinder bewertet haben. Dank gilt auch den Studenten, die das Bewegungstagebuch entwickelt haben. Darüber hinaus danke ich für die technische Unterstützung Thomas Dorloff.

Ein Dankeschön auch den Freunden, die sich als Versuchspersonen während der Entwicklung der experimentellen Versuchsanordnung zur Verfügung gestellt haben. Für wertvolle Gespräche danke ich auch meinem Großonkel Rudolf Richwien und für Literaturhinweise zu Jaspers danke ich Herrn Pfarrer Hermann-Josef Seideneck. Vielen Dank weiterhin Leonie Kraus sowie meinen Eltern für ihre dauerhafte Unterstützung. Besonderer Dank gilt meinem Mann Christian, Monika Kleinschmidt, Petra Otte, Anett Funke, Peter Fawcus sowie René Muschter für die Korrektur des Textes oder seiner Teile.

Meinem Mann Christian danke ich nicht zuletzt für seine immerwährende Unterstützung und Liebe. 


\section{Eidesstattliche Erklärung}

Ich versichere, dass ich diese eingereichte Dissertation „Wahrnehmung und Vorstellung von Bewegungen - Studien im Kontext des Erwerbs sportlicher Fertigkeiten in der Kindheit" selbständig und ohne unerlaubte Hilfsmittel verfasst habe. Anderer als die von mit angegebenen Hilfsmittel und Schriften habe ich mich nicht bedient. Alle wörtlichen oder sinngemäßen den Schriften anderer Autoren entnommenen Stellen habe ich kenntlich gemacht.

Sandra Salb 